Tiis report was prepared ats an acsount of work sponsored by an agency of the United Suates Government. Neither the United States Gorernment nor eny agency thereof, nor any of their employoes, makes any warranty, express or implied, or assumes any legal liability or responsibility for the accuracty, campleteness, or uscfulness of any information, apparatus, product, or precess disclosed, or represents that its use would not infringe privately owned rights. Reference hercin to any specific commercial product, process, of service by trade name. trademark, manufacturer, or otherwise does not necessarily constitute or imply its endorsement, recommendation, or favoring by the United States Government or any agency thereof. The views and apinions of authors expressed herein do not necessarily state or reflect those of the United States Government or any agency thereof.

\title{
OPTICAL TUNING IN THE ARCS AND FINAL FOCUS SECTIONS OF THE STANFORD LINEAR COLLIDER *
}

\author{
Philip Sébastien Bambade \\ Stanford Linear Accelerator Ceater \\ Stanford University \\ Stanford, California 94309
}

March 1989

Prepared for the Department of Energy under contract number DE-AC03-76SF00515

Printed in the United States of America. Available from the National Technica] Information Service, U.S. Department of Commerce, 5285 Port Royal Road, Springfield, Virginia 22161. Price: Printed Copy A08, Microfiche A01.

* Ph.D. thesis. 


\section{OPTICAL TUNING OF THE ARCS AND FINAL FOCUS SECTION OF THE STANFORD LINEAR COLLIDER (SLC)}

In this thesis, we present the experimental tuning procedures developed for the Arcs and for the Final Focus Section of the Stanford Linear Collider (SLC). Such runing is necessary to maximize the luminosity, by minimizing the beam size at the interaction point, and to reduce backgrounds in the experiment.

In the final Focus Section, the correction strategy must result from the principles of the optical cesign, which is based on cancellations between second order aberrations, and on the ability to measure micron-size beams typical of the SLC.

In the Arcs, the corrections were designed after the initial commissioning, to make the system more error-tolerant, through a modification in the optical design, and to enable adjustments of the beam phase-space at the injection to the Final Focus System, through a harnnonic perturbation technique inspired from circular acceltrators. Although the overall optimization of the SLC is not entirely finished, an almost optimal set-up has been achieved for the optics of the Arcs and of the Final Focus Section.

Beams with transve:se sizes close to the nominal ones, of a few microns, have been obtained at the interaction point. We present and discuss our results and the optical limits to the present performance. 


\section{ACKNOWLEDGEMENTS}

I would first like to warmly thank my third cycle thesis advisor, Jean Buon, who encolraged and helped me to get into the fascinating field of accelerator physics.

After my third cycle thesis, I had the opportunity to work on part of the design and commissioning of the first linear collider for high energy physics, at SLAC, Stanford, and clearly, it is SLAC, for its hospitality during my years as a visiting physicist, and my friends and colleagues, whom I want to thank the most. These four years were both extremely exciting and interesting.

Among the numerous people whom I worked and interacted with, I would like to thank especially Burt Richter and Rae Stiening, who encouraged me and made me work hard, Karl Brown, Andrew Hutton, Witold Kozanecki, David Ritson, and Nobu Toge, with whom I had the opportunity to collaborate, and from whom I learned an enormous amount, and the many physicists and engineers working on the SLC.

I present this thesis on the methods which we developed to commission a part of this new machine: the arcs und the final focus section. At the beginning of my stay at SLAC, and during most of my work, the lack of documentation resulting from our hectic schedules was often a big impediment. One of the motivations of my thesis is to contribute to this documentation.

Jacques Haissinski bas accepted to direct my work from Orsay, and his advise on the organization and writing of the manuscript was of great help to me. During his stay at SLAC, we also had the opportunity to be "on shift" together. The clarity of his thinking and his enthousiasm were often a guide for me. I would like to express all my gratefullness to him.

I would also like to thank Michel Davier for having presided my thesis committee, Karl Brown and Wolfgang Schnell for having travelled to be part of it, respectively from Stanford and from Genève, and Jean Buon and Bernard Grossetète, also for being part of jt.

Finally, I am very grateful to Jocelyne Brosselard, Nicole Mathieu, and A nnie Pottier, from the scientific secretariat, for their efficient help in putting my thesis together, Bruno Mazoyer, for his help on the figures, Alain Crueslan and Jacques Pennec, for the speed at which they got it printed. 


\section{INTRODUCTION}

I.1 LINEAR COLLIDER APPROACH AND BASIC NOTIONS

I.2 THE STANFORD LINEAR COLLIDER 2

1.3 SCOPE AND OUTLINE OF THESIS

\section{OPTICS IN THE ARCS AND FINAL FOCUS}

II.] OPTICS IN THE ARCS

Il.1.1 Overview

I1.1.2 Synchrotron Radiation

II.1.3 "Chromatic Filamentation"

II.1.4 Chromatic Correction

II.1.5 General Layout

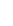
3

\1.2 OPTICS IN THE FINAL FOCUS

II.2.2 First Order Chromaticily 9

II.2.3 Chromaticity Compensation and $2^{\text {nd }}$ Order Distorsions 10

II.2.4 Origin and Scaling Laws for $2^{\text {nd }}$ Order Distorsions 11

II.2.5 Limits on Chromatic Correction Bend Angle $\quad 12$

II.2.6 Optimization Procedure $\quad 12$

II.2.7 Optical Bandpass

Il.2.8 Optimum $\beta^{*} \quad 14$

II.2.9 Gicneral Layout $\quad 14$

\section{IMPERFECTIONS AND TOLERANCES}

III.I INTRODUCTION 16

III.2 EFFECTS FROM IMPERFECTIONS 16

III.2.1 Errors in the Guide-Field and Beutron Oscillations 16
III.22 Errors in Focussing-Field and Phase-Space Disionions

$\begin{array}{lll}\text { III.3 EVOLUTION OF PERTURBATIONS - CORRECTIBILITY } & 17\end{array}$

III.3.1 Case or Chromaticity Corrected Optical Lataice 17

III.3.2 Zase of Lattice not Corrected for Chromaticity 17

III.3.3 Frequency Analysis 18

III.3.4 Electromagnetic Coupling to the Environment - Wake-Field Effects 20

III.3.5 Number of Free Parameters of Transverse Phase-Space 20

III.3.6 Classifrcation of Phase-Space Perturbations $\quad 22$

III.4 CONSEQUENCES ON PERFORMANCES AND SENSITIVITIES

III.4.1 Sensitivities Pertinent to the Luminosity 23

III.4.2 Sensitivities Pertinent to Packgrounds in the Experimental Apparauss 24

$\begin{array}{lll}\text { III.5 TOLERANCES AND TUNING : THREE MAJOR PIECES } & 27\end{array}$

III.5.1 Intmoduction $\quad 27$

III.5.2 Sources and Injector $\quad 28$ 


\section{SLC TURN-ON PROGRAM}

IV.1 INTRODUCTION

IV.2 EARLY COMMISSIONING PHILOSOPHY

IV.3 OPTIMIZING THE ARC PHASE-ADVANCE 32

IV.4 MODIFTCATION OF ROLL-BOUNDARIES

IV.S NEW AND TIGHTER CONSTRAINTS FROM BACKGROUNDS

IV.6 COLLIDING BEAM OPERATION

[V.7 TOO MANY KNOBS

$\begin{array}{lll}\text { IV.8 PRESENT GOALS } & \text { 35 }\end{array}$

\section{OVERVIEW OF OPTICAL TUNING IN} THE ARCS AND FINAL FOCUS

$\begin{array}{lll}V .1 & \text { INTRODUCTION } & 37\end{array}$

$\begin{array}{lll}\text { V.2 OPTICAL TUNING IN THE ARCS } & 37\end{array}$

Y.2.1 Phase-Adjustments 37

V.2.2 Smoothing out Roll Discontinuities

V.2.3 Harmonic Corrections 38

V.3 OPTIC.A: TUNING IN THE FINAL FOCUS

\section{REPORTS AND PUBLICATIONS ON OPTICAL} CORRECTIONS IN THE ARCS

V1.1 BETATRON PHASE-SPACE DIAGNOSTIC IN A FODO ARRAY (SLAC-CN-367) 55

V1.2 ROLL-FIX - AN ADIABATIC ROLL TRANSITION FOR THE SLC ARCS (SLAC-PUB-4835 Rev.)

VI.3 SPECIFICATION OF HARMONIC CORRECTIONS (WIREFIX) FOR THE SLC ARCS (SLAC-CN-370)

VI.4 FIRST TESTS OF HARMONIC CORRECTION METHOD

VI.4.1 Resonant Amplification of One-Dimensional Motion 128

VI.4.2 Separation of Horizontal and Vertical Betatron Frequencies $\quad 129$

VI.4.3 Other Perturbations and Empirical Adjustments $\quad 129$

$\begin{array}{ll}\text { VI.4.4 Conclusions from the First Tests } & 130\end{array}$

\section{REPORTS AND PUBLICATIONS ON OPTICAL} CORRECTIONS IN THE FINAL FOCUS

VII.1 ORTHOGONALITY OF FINAL WAIST CORRECTION AT THE IP OF THE SLC (SLAC-CN-369) 
VII.3 FIRST ORDER OPTICAL MATCHING IN THE FINAL FOCUS SECTION OF THE SLAC LINEAR COLLIDER (SLAC-PUB-4621)

VII.4 OPERATTONAL EXPERIENCE WITH OPTICAL MATCHING IN THE SLC FINAL FOCUS SYSTEM (SLAC-PUB-4776)

VIII. REPORT ON GENERAL STATUS

VIII.1 RECENT PROGRESS AT THE STANFORD LINEAR COLLIDER (SLAC-PUB-4610)

\section{CONCLUSION}

\section{APPENDIX : REPORTS AND PUBLICATIONS ON THE BEAM-BEAM DEFLECTION DIAGNOSTIC METHOD}

A.1 BEAM-BEAM DEFLECTIONSV TO MEASURE SPOT SIZE AND OFFSETAT THE SLC IP (SLAC-CN-303)

A.2 BEAM-BEAM DEFLECTIONS AS AN INTERACTION POINT DIAGNOSTIC FOR THE SLC (SLAC-PUB-3979)

A.3 OBSERVATION OF BEAM-REAM DEFLECTIONS AT THE INTERACTION POINT OF THE STANDARD LINEAR COLLIDER (SLAC-PUB-4767) 


\section{List of Figures}

Page

1. Average radius of the majority of the storage rings as a function of their energy. The dependance is close to quadratic.

2. The Stanford Linear Collider 40

3. General layout of the arcs. The numbers refer to each achromat 41

4. Optical functions of the arc lattice : in (a) the $\beta$-function, and in (b) the n-function

5. Rolls about axis of achromats in north and south arcs

6. Longitudinal displacement of the focal point with the energy

7. Variation of the $\beta$-parameter with the fractional energy error in the final focus section, with churomaticity correction (a), and without chromaticity correction (b). As can be seen, the optical bandpass of the system is enlarged by the chromaticity correction.

8. A simplified final focus section

9. Relative variation of the luminosicy with the $\beta^{*}$-parameter in the final focus section, without chromaticity correction, and for several values of the energy spread.

10. Contribution to the interaction point beam size of the main second order abberrations, as a function of the bend angle of the chromatic correction section (2), of the distance between the last quadrupole and the collision (b), of the length of the chromatic correction section (c), and of the total length of the system (d). The real power dependances for each of these abberations are :

(a) $\mathrm{T}_{1266} \propto \mathrm{B}^{0}, \mathrm{~T}_{1446} \propto \mathrm{B}^{-1}, \mathrm{~T}_{1244} \propto \mathrm{B}^{-2.05}$

(b) $T_{1 \text { ijk }} \propto(\ell *) 1.79$

(c) $T_{1266} \propto\left(l_{\text {ccs }}\right)+0.86$

(d) $T_{1 \mathrm{ijk}} \propto\left(\ell_{\text {tot }}\right)^{-0.98}$

In the case of (c), the power dependance is slightly weaker for $T_{1244}$ and for

$T_{1446}$ for large values of $\ell_{\text {ccs }}$.

11. Relative variation of the luminosity with the $\beta$ *-parameter, with chromatic correction (full line), and whithout chromatic correction (dotted line)

12. Schematic of the final focus optics. Correctors for betatron matching are shown shaded, and for dispersion matching cross-hatched

13. Betatron oscillation (a) and oscillation of the square of the beam envelope (b) in the focussing lattice. The oscillation of the square of the envelope is at twice the betatron frequency 
15. Incoherent imaging of a betatron oscillation of a beam with finite energy spread (a). This result in an abnormal correlation between the transverse coordinates and the energy of the particles in the beam. The projection of the phase-space $\left(x, x^{\prime} \frac{\delta E}{E}, z\right)$ on the plane $\left(x, x^{\prime}\right)$ is thereby enlarged

16. Incoherent imaging of a phase-space distortion. In (a), we show the nominal phase-space, and in (b) the distortion of this phase-space from a focusing error. The incoherent imaging of this distortion generates a second order correlation between the transverse coordinates and the energy of the particles. When this correlation becomes large, the projection of the phase-space on the plane $\left(x, x^{\prime}\right)$ will correspond to the larger cercle in which the ellipse is inscribed.

17. Basic principle of collimation

18. Illustration of the separation in three major pieces of the tuning in the SLC. There are two breakpoints in the accumulation of the ertors, from the radiation damping in the rings, and, in a more imperfect way, from the collimators at the end of the linear accelerator

19. Sequential application of the ten optical adjusments necessary to minimize the interaction point beam size

20. Resonant growth of the horizontal betatron oscillations, from a focusing perturbation at twice the betatron frequency

21. Coupling into the vertical plane corresponding to the oscillation in figure 20

22. Shift in verical betatron frequency from systematic perturbations of focusing and defocusing magnets with opposite signs

23. Transfer of horizontal betatron oscillations, and coupling into the vertical plane, for four initial conditions corresponding to phases separated by $45^{\circ}$, after optimization of the harmonic correctors

24. Transfer of horizontal betatron oscillations, and coupling into the horizontal plane, for four initial conditions corresponding to phases separated by $45^{\circ}$, after optimization of the harmonic correctors

25. Empirical adjustments of the beam size at the end of the arc, on two occasions ((a) et (b)). The photographs on the right show the beam before correction, and those on the left the beam after correction 


\section{List of Tables}

page

1. Main design and achieved parameters of the SLC. The achieved parameters corresponds to the fall of 1988

2. Classification of phase-space distortions which affect the luminosity in the SLC 
I. INTRODUCTION 


\section{I.1 LINEAR COLLIDER APPFOACH AND BAS:C NOTIONS}

In the past twenty years, electron-positron colliders have establisheol themother as powerfid touls for studying high-energy particle physics.

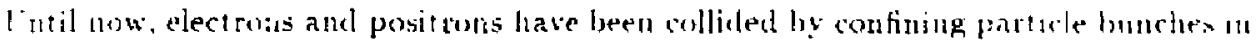
storage rings. This approach can however not he extrapolated as desis d tu crity hidi eneigy because of the rapid increase in simchootron radiation entuted in the bomling magnets. Tire energy losis per turn to synchrol ron radiation scales as $E^{4} R$, where $E$ is the beam energy and where $R$ is the average radius of the slorage ring. ic can be shown that because of ihis, both the size and the cost of a storage ring with an opultuzed design scale as $E^{-}$. This law is illustrated in Fig. 1. where the average ratius of unost electron-positun storage rings is shown as a function of thenr energy.

In alternate method for prociucung electron-p.ssit ron collisions consists of collirling beams of two npposed linear acceleralors'. In this rase there is no signiticant synchrot ron radiation except in the vicinity of the collision point. and thus nore favorable scaling with energy may br possible.

In order in be a useful tool for particle physics. an electron-positnon collider must in ardation to high energy also provide high luminosity - or event rate for a process with unit cross-iectun. The luminosity can be expressed as a function of the frequency of the $\therefore$ ollisions $f$. the muber of particles per bunch $V$, and the effective transterse area $A$ over which the collisions take place:

$$
\mathcal{L}=\frac{f I^{2}}{1}
$$

Present teclinology limis the re peition rate of linear accelerators lo a few hundred $\mathrm{Hz}$. This is much less than the fres,uencies ot secral hundred $\mathrm{KHz}$ at which hutches circulate in typal sturage rings. Aiso, the lunch population is usually higher in a storage ring than in a linear accelerator. In ordes f s rench comparable or higher luminusity. lhe transverse area of the beamb at the collision point of a linear collider must be made substantially. - Maller llaci that spical of storage rimgs:

The collision point area $A$ is comvenienty expressed as a function of $x$ the flansterse

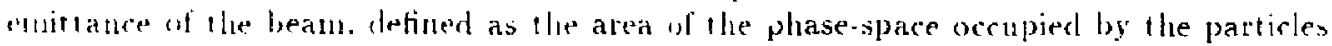

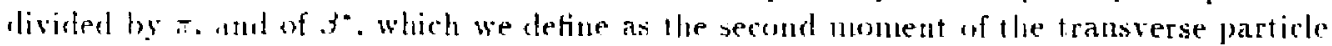
clistribution at the coll: jion goint mormalized on this emittance". If these two paratteters

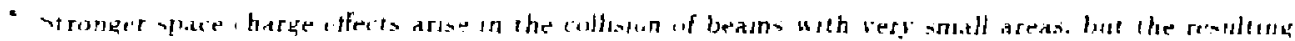

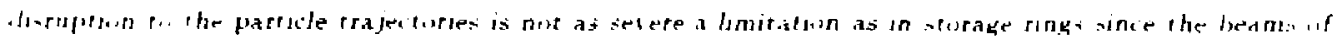

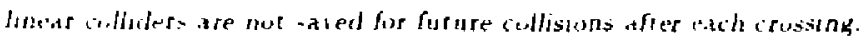

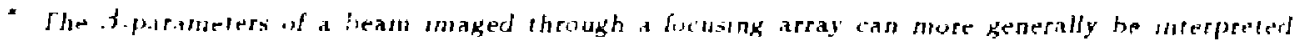


are the same in each transverse plane, one obtains for $A^{3}$ :

$$
A=4 \pi \beta^{*} \epsilon \text {. }
$$

The factor 4 in (2) arises through the overlap of the particle distributions. assumed to be gaussian in this calctlation.

To produce a small interaction area $A$, both the emittance and the $3^{\circ}$-parameter must be small. The enittance can only be made small at the source, of througli radiation - damping, which can be used to cool the beams in a dedicated storage ring. Such a storage is referred to as a "damping ring". The $3^{*}$-parameter can only be redured by focusing the beam to the snallest possible size. This is achieved in a dedicated optical system immediately upstream of the collision point. which must in general be corrected for chromatic aberrations. This optical system is referred to as a "final focus system".

Besides the basic design of the components of a linear collider, the handling of imperfections in the long open structure iuvolved presents conceptually new problems. Such handling must be folded into both the detailed design of the individual components and into the overall system optimization. Considerable effort is in fact required at several stages of the system to preserve the carefully damped emittance and to precisely monjtor and control beam parameters. Both are necessary conditions for the final focusing to work properly and to minimize backgrounds in the experinental apparatus from secondaries produced by tite beam halo and by the tails which are typical of linear accelerators. Solving such issues at reasonable cost appears to be a crucial element in the developuent of inear colliders which must be factored into basic scaing and feasibility argunents.

\subsection{THE STANFORD LINEAR COLLIDER}

The Stanford Linear (ollider (SLC') is the first linear collicler presently operating. It uas conceived and built with the double motivation of :

1. Producing high luminosity elect con-pusitron collisions whth a center of mass energy of about 92 (ieb, to study the plysics of the internediate vector boson $Z^{0}$.

2. Providing the first practical test of the linear collicler approach tuwards high energy: elect ron-posit ron mactines.

A schematic of the SLC is shown in Fig. 2. In the case of the SLC' one single linear acceleratol - the Stanford linear accelerator - is used rather than two opposing systents

as the values raken by an envelope function $f(s)$ This function rescribes the esidution ihrough the array of the square of the beam size curresponding to a phase-space with emitance unity . Wore un rlle correspondanic berween these two definitions san be found in sertion III 
as was described in I.1. to accelerate both electron and positron beams. The beams are then ained into collision by means of two ares. The collision point bean size reguired to, achieve significant luminosity is nominally a lew mucrons.

More specifically, at the begining of each cycle. two elect ron bunches are generated and co-accelerated to $1.116 \mathrm{GeV}$ in the injector and in the first sector of the linear accelerntor'. At $200 \mathrm{Mel}$, they are joined by a positron bunch. The three are then injected tor comling into two damping rings ${ }^{\circ}$. from which they are extracted before the next linac pulse. They: are 1 ken reinjected into the linear accelerator and co-accelerated up to enelgies of 50 Cete ${ }^{7}$. The $6 \mathrm{~mm}$ equilibrium ring bunch-length is compressed to $1.5 \mathrm{~mm}$ before reinjecting into the linear accelerator to minimize wake-field effects produced in the accelerator wave-guile structure by the intense bunches. The last electron bunch is ejected onto a target at $3: 3$ Gev. 10 produce positrons ${ }^{8}$. These are returned along the length of the Linar to the 200 Hev point in the injector. At the end of the linear accelerator. electrons and positrons are bent around and into collision through tho arcs". The final focus system. st raddling the interaction area. provides the necessary optical domagnification and steers the beams into collision ${ }^{10}$

The main parameters of the SLC are shown in Table 1 . Column one lists the desinn paratueters. and column two lists presently achieved paranteters. As can be seen the luuknosity reaches unfortunately only a thousaudh of the nominal value. Also, the vperaling efficiensy for achieving peak lummosity with acceptabie background in the experintental apparatus (not listed in Table 1 ) was lower than $5 \%$ in the last cycle of luninosity runs during the suntmer of 1988.

The discrepancy between expected and achieved performance results parly from insufficient e:uphasis, during the early phases of the project, 0 ? the design of systematic diagnostic and correction schemes to control the beams adequately at each stage of the systen, on ihe development of a global integrated tunjug strategy and on hacdware reliability and stability. Tuning and correction net hods were in most cases cleveloped empirically aud added-on to each of the components of the SLC after the basic optics and gemetry had been fixed. or sometimes in response to specific difficulties rucumbered in the conthnissioning phase. In several cases. the methorls deseloped were therelore imperfiect. ambl their improvenent is the centerpoint of the anguing effurt to bring the SLC tamarals its design performance. The experience gained with the SLC" which is she first hinear collicher w be lirought into operation. will be very useful to the design of future hintar conlicters.

\section{I.3 SCOPE AND OUTLINE OF THESIS}

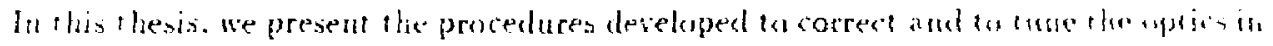

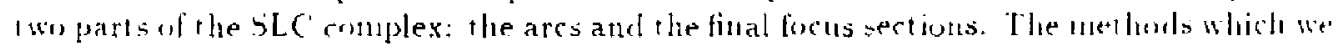


describe were conceived and developed in preparation for and during the commissioning of the SLC, mainly between 1984 and now. They are presented through a selection of technical wotes, conference articles and publications describing in detail their design and the operational experience which has been acquired. Although the entire SLC complex has not yet reached its nominal performance, these methods have enabled to achieva a close to optimized optical transport in these two sections. and to characterize the optical limitations to the SLC performance.

He introduce these papers in an overall presentation where we:

1. Review the basic principles of the optical designs of the arcs and final focus sections: on which our tuning methods are based.

2. Study the effect of focusing errors in the systen on the luminosity and on the background. These errors can result in both recoverable and unrecoverable distortions of the beam phase-space. We give order of magnitudes for resulting tolerances and characterize the different cases in terms of their effect on the phase-space and of their correctability.

3. Describe the context of the SLC commissioning, and the evolution of the global runing strategy. from which the optimization of the arcs and final focus can not be entirely isolated. Some of the methods were developed in preparation for the commissioning, while others were conceived in response to specific difficuities encountered during the first tests.

We then introduce each of the papers presented, show its relevance to the subject of this thesis. and out line its original components.

In the case of the arcs, the papers presented fescribe essentially:

1. A method for correcting long FODO arrays in a compact and economical way. This uethod is inspired from harmonic correction techniques common to circular accelerators. Its application to an open beam-line is new. We have designed and implemented this niethod in the last portion of the arc beam-line, to enable tuning the phase-space at the entrance to the final tocus section. Such tuning has turned out to be crucial to uniniuze backgrounds resulting in the experimental apparatus from seccndary particles. Such secondaries are generated when edges or tails of mismatched beams get scraped off in the tinal focus system, where the aperture (normalized to the nomulnal beam size) is sicuificaurly smaller than in the arcs.

2. The study of the partial moditication of the arc lattice. To follow the terrain of the SLAC' site. the ares were designed in three dimensions: in arddition to the horizontal bendiug. the bean-line is dettected vertically by rotating the beuding nagnets about their axis. In the initial resigu. these rotations were iutroduced abruptly. The coupling between the hurizontal and vertical betat ron motions was suppressed by grouping these rotatjotis in lung-ange cancelling pairs. This strategy. hovever, made the ststem sensitive to systematic errors. It has been possible to modiry the design to produce sumother transitions. illese new transitions almost entirely suppress the sensitivity to systematic errors. and 
render the overall optical transport much more tolerant.

In the case of the final tocus. the papers presented describe essentially:

1. The elaboration of the interaction point beam size minimization procedure. This procedure consists of the sequential application of ten corrections in lle different optical modules of the final focus section. following a strategy which must take into account the ability to measure very small beam sizes and to resolve the various distortions. as well as the specificity of the tinal focus optics. The final focus design is in effect based on balancing several higher order optical aberrations which can individually donunate the minimum beam size if the system is not adjusted correctly. It is now possible to adequately cont rol the interaction point beam size, starting with a very distorted plase-space at the entrance to the system.

2. The practical implementation of this procedure and the operational experience which has been acquired, along with the present optical factors which linut the SLC luminosity. As we explain, these optical limits result principally from the requirenent to minimize backgrounds in the experimental apparatus, and from a somewhat larger than nominal euit tance at the exit to the SLAC linear accelerator.

Finally, we include. in an appendix, papers on the development and on the initial performance of nue of the three measurenent techniques. based on electronagnetic spacecharge effects occuring in the collisions bet ween the two beaus, which wete conceived to diagnose the beams at the interaction point: the "beam-beam deflection" technique. The two ot her methods which were developed are based on "beamstrahlung", or synchrotron radiation from the space-charge forces, and on "disruption". Such methods are particularly important to diagnose the beams at the interaction point. Iut do not belong directly to rle subject of llis thesis. 


\section{OPTICS IN THE ARCS AND FINAL FOCUS}




\section{II.1 OPTICS IN THE ARCS ${ }^{11}$}

\section{II.1.1 Overview}

The arcs are the only part of the SLC which will not be required in linear colliders of the future. They are needed in the SLC system, which uses the same linear accelerator to accelerate both beams, to bend each beam around $270^{\circ}$, and into frontal collision. Because of the linited size of the SLAC site, they have a short bending radius of 279 meters. They are intended to behave passively and to produce a close to exact optical image of the electron and positron beams injected from the linear accelerator. The optics is therefore designed to be an optical itentity from the beginning point to the exit. A schematic of the two arcs is shown in Fig. 3. Two plysical mechanisms must be counteracted to prevent the beam phase-space from being enlarged as it is imaged througli: synchiotron radiation enit tance dilution and filamentation effects from the natural chromaticity of the lattice anci from the fact that the bean has a finite eilergy spread.

\section{II.1.2 Synchratron Radiation}

Syuchrotron radiation photons are emitted at random and causr energy fluctuations. Lower energy particles are bent more and follow curves with shorter average radius. This disperses their trajectories incolerently which in I urn dilutes the phase-space in the plane of the bending. Such trajectorjes execute betatron oscillations in the quadrupole lattice. To minimize the growth. both the photon emission rates aud the oscillation anplitudes nust be suall. This is achieved by making the bending radius large and the betatron period short, through tight focussing. It can be shown that the giowth is proportional to $T_{, 3}^{3} / \rho^{4}$, where $T_{3}$ is the betatron period and $\rho$ the average radiust. The transport therefore nses the lowest possible field compatible with the size of the $S I A C$ 'site, and a strong fccusing alternating gradjent lattice, with optical parameters chosen to uuiuuze the average invariant amplitude of the clispersed ascillations. For a FODO array, the optinum cell phase-shift ${ }^{12}$ to minimize enittance growth is near $135^{\circ}$. For reasons explained below. the design in fact uses $103^{0}$. The packing factor is in addition maximuzed by using combined function magnets.

At 50 Gev, the enittance added in one passage is $1.310^{-10}$ rad-m for one half of the tesign value at the entrance) in the horizontal plane, and $0.510^{-10} \mathrm{rad}-m$ in the vertical plant ${ }^{t}$. The enittance growth occurring in the vertical plante arises becanse of vertical dinpersion introduced by the rolls (described tater).

\section{II.1.3 "Chromatic Filamentation"}


The second mechanism for phase-space growth arises through residual energy spreacl resulting from the bunch-length and the acceleraling linear accelerator RF. Because of the energy dependance in the focussing. or chromaticity. optical distortions at the injection to the arc. or arising from gradient errors within the arc lattice, are not imaged coherently. For example an off-energy shice of a mismatched phase-space is transmitted with a phaseshift $\Delta_{2}=2 \pi \gamma_{3} \frac{d \sigma}{d G} \delta_{E}$ where $\varepsilon_{E}$ is the relative energy etror, $x_{3}$ is the number of betatron periods. and $\frac{d \Phi}{d G}=1.5$ is the ratio of phase change to excitation change in the arc lattice. For large phase-shifts, the overall mismatch areraged over all energies looses its phase relation to the input. The effective volume occupied by the observable pliase-space is thereby enlarged. This effect is referred to as "chromatic filanentation". Enlike the phase-space dilution resulting from synchrot con radiation. this enhancement of the effective volume does not violate Liouville's Theorem. The volume of the full six-dinensional phasespace $\left(x, x^{\prime}, y \cdot y^{\prime}, \frac{\delta E}{E}, z\right)$ is in fact not changed, but acquires a complicated internal structure corresponding to a second order correlation.

The arc beam-lines have $\mathrm{N}_{3}=69$ betatron perinds. For a tractional energy spread of $\sigma_{E} / E \simeq 0.5 \%$. the spread in betatron phases at the output is about $\sigma_{\psi} \simeq \pi$. In order to assure colerent imaging of focusing srrors injected into the arc or generated along the beam-line, over an energy band-pass of $\pm 0.5 \%$, the design uses sext upoles to cancel the first-order chronaticity of the lattice.

\section{II.1.4 Chromatic Correction}

Tise sextupoles are int roduced by shaping the combined function nagnet poles ${ }^{7}$. For the horizontal opties, the vertical component of the magnetic field on the horizontal axis $(y=0)$ is:

$$
B_{y}(x)=B_{y o}\left(1-\frac{Q}{\rho} \cdot x+\frac{S}{\rho^{2}} \cdot x^{2}\right)
$$

where $Q$ and $S$ are the strengt hs of the quadrupole and sext upole componeat s respectively. and $\rho$ is the bending radius. The sext upole provicles additional focusing finc off-energy and off-axis rays with $x=\left\{x-\delta_{E}\right\rangle$, where $\eta$ is the dispersion function. which suppresses the chromaticity if $2 S \eta=Q \rho$. Since sine and cositue-like components are equicalent moduln $\pi .2$ in a repetitive lattice. only one family per plane is needed. Addlitional terms in $r^{2}$ and $h^{2}$ for rays solely off-axis or off-energy are suppressed by grouping the cells to produce cancellations as hocally as possible. Secund orcher arlironats achieve this by pairing the sextupoles $\pi$ phase-shift apart and by requiring at least four-fold sequential symmetry ${ }^{13}$. This is clone by grouping the cells into achromatic superperiods wit the suallest pusibible nutu ple of $2 \pi$ compatible with the cell phase-shift. In the SLC ares. each surh =uperperiod ur achromat - spans a bT phase-adrance and coth sist of ten $10 x^{0}$ cells. This: is a compronuise bet ween achromat compact ness, best with $90^{\prime \prime}$. and quant um dilut inn. matlest near $133^{\prime \prime}$. The resulting latrice functions 3 and $\eta$ are shown in Fig. 4. 


\section{II.1.5 General Layout}

The whole arc consists of 23 such achromats. In adidition, as shown in Fig. 3. specia] matching sections are used at the inflexion points (reverse bends sections) and between the linear accelerator and the main arc (bean switchyard section). The matrhing sections needed at tue exit of the arrs are described in the section on the final forus.

Finaly, for economical reasons, the arc beam-lines are required to follow the terrain of the SLAC' site, and are therefore not planar. To provide the necessary vertical deflections. the achronats are rolled relative to each others. The tistribution of rolls for the north and south arcs are shown in Fig. 5 .

\section{II.2 OPTICS IN THE FINAL FOCUS ${ }^{14}$}

\section{II.2.1 Overview}

The final focus is the lait section of bean-line in the SLC before the interaction point. Its main function is to focus the beam to a Iransverse size of less than $2 \mu \mathrm{ml}$ at the collision point .

Schematically the final focus consists of a demagnifving telescope, simular to a low-3 insertion in a circular machine, with a special section at its entrance to natch the arc lattice paraneters. Because of the finite enuttance and monentum spread in the beam. a $2 \mu m$ beam spot cannot be obtained without carefully minimizing ligher orcler clicomatic aud geometrir aberrations. The dominant optical aberration is the first order chromaticity - or momentum dependance of the locusing - of the demagnifying telescope. Ptysically. this first order chromaticity causes particles with different energies to be imagerl into longitudinally displaced focal points at the interaction point. The unguitude of this effect is easy to estimate by considering solely the cariation with energy of the focal length of the last foctusing elements. Since this focal length. computed as the distance form the interartion point to the principal planes of the final lens system itl eark plane, is about 5 merers. and because the depth of focus of the optical system. measured by the linear 3-function at hie interaction point is rectuired to be about 0.5 centimeters, we see liat particles with energy errors of a few parts per thomsand are totally out of focus i See fing. b)

\section{I1.2.2 First Order Chromaticity}


More quantitatively. we use TRANSPORT ${ }^{15}$ to compute the rate of change $F$ of the interaction point beam size with the fractional energy error $f_{E}$. We have:

$$
\sigma^{2}\left(\delta_{E}\right)=\epsilon \jmath^{*}\left(\delta_{E}\right)=\epsilon 3_{0}^{*}+\frac{\epsilon}{3_{0}^{*}} F^{2} \delta_{E}^{2}
$$

where $3_{0}^{*}=3^{*}\left(\delta_{E}=0\right)$ represents the nominal value of the $3^{*}$ parameter, and where $\epsilon$ is the emittance. One obtains $F \simeq 15 \mathrm{~m}$. where $F \simeq 15$ ineters, where $3_{\mathrm{c} f f}^{*}$ and $J_{l, n}^{*}$ are the effective and linear 3 -functions at the interaction point.

The presence of this first order* chromatic term in (4) leads one to define an energy band-pass over which the demagnifying telescope will perform its function. This band-pass is shown in Fig. Ta. As can be seen it is niuch smaller than the minimum fractional energy spread of 0.002 which can presently be obtained" at the end of the linear accelerator.

An effective value could be calculated for the $3^{*}$ parameter, by averaging equation (4) over the particle's energy dist ribution. This parameter would however not represent the new particle distribution very well since this new distribution is more peaked than a gaussian. and has tails. Rather than calculating this new distribution explicitly, we will directly estimate the effert on the luminosity, by averaging the usual expression over the two beam's energy distributions, which we take to be equal and square. One obtains:

$$
\mathcal{L}\left(3^{*}, \sigma_{E}\right)=\frac{f X^{2}}{2 \pi \sigma_{E}^{2}} \int_{0}^{\sigma_{E}} \int_{0}^{\sigma_{E}} \frac{d \delta_{E^{+}}^{+} t \delta_{E}^{-}}{2 \epsilon^{*}+\frac{\epsilon}{\xi^{2}} F^{2}\left(\delta_{E}^{+2}+\delta_{E}^{-2}\right)}
$$

The result is shown in Fig. 8 for different energy spreads and allows to determine an optimum $3^{*}$. Since this en rgy band-pass scales as $j^{*}$ from (4), and because the optimum occurs when the band-pass is maiched to the energy spread, one expects the optimum $3^{-}$ to scale roughiy linearly with the energy spread in Fig. 8. This can indeed be verified.

\section{II.2.3 Chromaticity Compensation and $2^{n d}$ Order Distortions ${ }^{14.4}$}

The first order chromaticity is corrected by introducing a special clirontatic correction section upstream of the demagnifying telescope (see Fig. 9). This chromatic correction section consists of two - I telescopes, combined with chipoles at their foci, to generate signiticant energy dispersion at the quadrupoles. Sextupoles. where the focusing strength varies linearly with excursion, are put near the quadrupoles. to provide additional focusing proportional to energy. This compensates for the intritsic first order chromaticity. Additional first-order perturbations to the imaging produced by each sextupole are made to cancel iner the length of the section by pairing the sextupoles $\pi$ betatron phase-shift apart

* The chromatk term in question appeats to be a second order term in (1). but it is really dies: cursesfund tu a first order term in the optical ransfer since (A) gives the behaviour of the scurare of the beam size 
and by imposing sequential symmetry for the dispersion function. In this way, all residual perturbations to the focusing are pushed to second order. The parameter $3^{*}$ can thus now be written:

$$
3^{*}=3_{0}^{*}+\kappa_{1}^{2} \frac{\delta_{E}^{*}}{3_{0}^{*}}+\kappa_{2}^{2} \epsilon \frac{\delta}{E}_{3_{0}^{2}}^{2}+\kappa_{3}^{2} \frac{\epsilon^{2}}{3_{0}^{* 3}},
$$

where $s_{1}, n_{2}$ and $n_{3}$ measure the magnitudes of the residual second order perturbations to the focusing.

\section{I1.2.4 Origin and Scaling Laws for $2^{\text {nil }}$ Order Distortions}

Because the SLC is designed for round beams at the collision point, with equal enittances and 3 -functions in each plane, the linear chromaticity must be cortected in both planes. and two families of sextupoles must be used. In order to save space, a single cliromatic correction section, with interleaved fanilies for correction in each plane, is used. The second order perturbations to the focusing come in most part from coupling between these two interleaved families. This consideration allows to develop apprcximate scaling laws for these termis ${ }^{16}$.

Since the largest contribution to the first order chromaticity cones from the final dentagnifying telescope, we can find a mearly exact scaling law for the strengths of tha sextupoles by neglecting the first order chromaticity introduced by the telescopes which make up the chromatic correction section; the scaling law is obtained by equating the first order chromatic contributions to the variation of the interaction point ang iar spread, from the intrinsic first order chromaticity of the final demagnifying telescope and from the sext upoles. Wie obtain:

$$
S \times \frac{l \cdot \frac{1}{l_{e c},}}{B},
$$

where $S$ is the strength of the sextupoles, $l^{*}$ is the distance from the interaction point to the principal planes of the last lenses. $B$ is the angle of bending in the chromatic correction section, and $i_{\text {ecs }}$ is the length of the chromatic correction section (see Fig. 9).

Xext, because the dominant second order perturbations to the focusing in (6) come from the cross-coupling of the sextupole families used to correct in each plane, we can neglect the final demagnifying telescope in the scaling laws for these second order teruns. We find in thus case:

$$
\left\{\begin{array}{l}
n_{1} \times S^{2} l_{c c s}^{3} B^{2} \\
n_{i} \times S^{2} l_{c c s}^{3} B_{1} i \\
n_{3} \times S^{2} l_{c c s}^{3} M^{2}
\end{array}\right.
$$

where $I I=l^{\prime}, L$ is the magnification of the final denagnifying teiescope. and where $L$ is the distance between the lens and the "source" in Fig. 9.

By substituting in ( 8$)$ for the sextupole strength needed to correct the first orclet clemuaticity in (T). we find the lollowing scaling laws for the three contributions to the 
interaction point beam size which arise from the second order terms in (6):

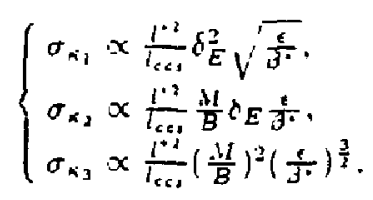

It can in acldition be noted that scaling $l^{*}$ at corstant $. M=l^{\circ} / L$ is equivalent to scaling the full length of the final demasnifying telescone". Thus if we scale the whole length $l_{\text {tot }}$ of the final focus system, we expect all tems in (9) to scale linearly.

The dependence of these three limiting residual second order terms on the design variables: $B, l_{c c s}, l^{*}$ and $l_{\text {tot }}$ has been checked with TRANSPORT, by scaling the SLC final focus design. The restults of such calculations are shown in Fig. 10a,b,c,d.

As can be seen the scaling is not perfect. This arises mainly because the first order chromaticity of the lenses in the chromatic correction section is not, as was pointed out, included in the scaling argument. Because of this. the reduction of the magnitude of thesa terms when increasing the length $l_{c r}$ of the chromatic correction section is less than linear and eventually saturates, in particular for the geometric term $\sigma_{n_{3}}$. Similarly, the reduction of their magnitude when decreasing $l$ is less than quadratic. The actual sinulated power dependances are given in the figure captions.

\section{2.5 Limits on Chromatic Correction Bend Angle}

As can be seen from (9), there is a lower limit on the angle $B$ of the bending in the chromatic corcection section, from both the chromatic term in $h_{2}$ and from the geometric term in $\kappa_{y}$. We will return to this point in the next section.

In addition. there is a sharp upper limit on this bend angle from syuchrotron radiation emittance dilution, which increases as the fifth power of the bend angle and if the beam energy. For the SLC final focus. the limit occurs ${ }^{14}$ when this bend angle $B$ is of the order of about one degree, which corresponds to all emittance growth of about $20 \%$ at 50 (iel.

\section{II.2.6 Optimization Prosedure}

The scaling laws given in (9) allow one to uncterstand the different procedures which cau be followed to optimize the optical design. A full description of the optinuzation process can be found in Ref. i6! .

\footnotetext{
* By length of the telescope. we intend the distance between the image and the source
} 
Several points of views can be taken. Here, we will show one of them, whici consists in considering that the emittance $\epsilon$ and the energy spread $\delta_{E}$ have been set in the werall system specification, that a given $\beta^{*}$ is desired, and that ous wishes to choose the lengths $l_{\text {tot }}, l_{\text {ces }}$ and $l^{*}$, and the bend angle $B$ in an optimal way*.

A reasonable procedure would go as follows:

1. Set the bend angle $B$ to the maximum possible consistent, from the point if view of the syrchrotion radiation enittance ditution, with the chosen minimum beam size $\sigma^{*}=\sqrt{\beta_{0}^{*} \epsilon}$.

2. Set the magnification $M$ such that all terms in $(9)$ be about equal, for the given emittance and energy spread, and for the chosen $3_{0}^{*}$. When this has been clone, it can be seen from the two first equations in (9) that the betatron and chromatic angular spreads at the input io the chromatic correction section are about equal.

3. Decrease the ratio ${ }^{* 2} / l_{\text {ces }}$ until the second order aberration terms and the linear term in (6) are about equal. This determines a minimum for $3^{\circ}$ in (6). As we will also see, this optinum can also be interpreted as the point for which the energy band-pass of the corrected system is matched to the energy spread $\delta_{E}$ of the heam.

Implicit in this is the fact that the sextupole strengths are free parameters. Also, the magnification. II of the final demagnifying telescope is in general not sufficient to chemagnify the input 3 -function to the desired $\mathcal{B}_{0}^{-}$, and an initial demagnifying section is neted upstream of the chromatic correction section. Therefore, when $M$, the magnification of the final demagnifying section, is varied, we assume that this initial demagnifying section can be adjusted to keep the overall magnification of the bean-line (and thus $\beta_{0}^{-}$) ronstant, and that over this adjustuent range, this initial section does not contribute signiticant!y to the chromaticity of the overall beam-line.

In practice, the minimum distance ${ }^{*}$ between the interaction point and the principal planes of the last focusing elements is set by the maximum gradient available in tlose focusing elements, and by the requirement to leave sufficient space for cmuponents of the experimental apparatus. In the case of the SLC final focus system, $l^{*} \geq$ inm. The optimization led to int coduring a denagnification of a factor four in bot lt planes for the final telescope. and of factors eight and three in the horizontal and vertical planes respectively for the initial telescope. Besides initiating the demagnification. the intial tramsfonmer also serves to match the final focus and arc betalcon latlicest. This results. given an energ.

* Twu different points of riew wuuld consist in asking:

1. Fur given constraints on the lengths log and $l^{*}$, what are the requirements un the emitcance alld an the energy spread w reach a giten it" ?

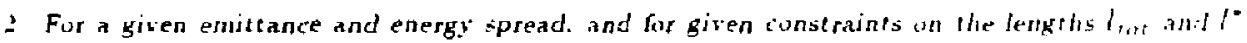

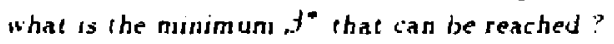


spread of $\varepsilon_{E}=0.002$ and an emittance of $\epsilon \simeq 310^{10} \mathrm{rad}-\mathrm{mu}$, to about a $j_{m_{11}}^{-}=4 \mathrm{~mm}$ (this is also illustrated in II.2.8). The bend angle $B$ is set to about one degree as was mentiontled above.

\section{II.2.7 Optical Bandpass}

The overall effect of the chromatic correction is to broaden the energy band-pass. described above. over which rays are imaged to the same interaction point focal point. From (5), the width of this band-pass senles roughly as $\sqrt{\beta_{0}^{*}}$ (if only the term in $n_{1}$ from (6) is use -1$)$. The bandpass is shown in Fig. ib. Defining it quantitatively as the band of energy deviations for which $\delta^{*} \leq 1.253_{0}^{*}$; it is $\pm 0.5 \%$ for $\beta_{0}^{*}=16 \mathrm{~mm}$ and $\pm 0.22 \%$ for $3_{0}^{*}=4$ nunl.

\section{II.2.8 Optimum $3^{*}$}

Much as in the case without chromatic correction described above, the lumino:ity is maximized by matching this enlarged band-pass, defined by , $3^{\circ}$, to the beam energy spread. This is illustrated in Fig. 11, where the luminosity loss is computed as a function of 3 for two different energy spreads, using the raytracing code $M\left[{ }^{\prime} R T L E^{17}\right.$. The soldd lines indicate the luminosity loss as a function of $3^{*}$ for the chromatically corrected system, and the clashed lines, reproduced from Fig. 8, show for comparison the loss which occurs without the chromatic correction. As can be seen the wider band-pass produced by the chromatic correction results in a smaller uptimum for il". $^{*}$.

\section{II.2.9 General Layout}

The whole system is shown in Fig. 12. Ir includes, in addition to the two telescopes and in the chromatic correction, a section to match the arc dispersion sunction. Also the extraction of the spent bean is built in to the upper telescope. using a kicker and septum magnet. 


\section{IMPERFECTIONS AND TOLERANCES}




\section{III.1 INTRODUCTION}

The goal in this chapter is to describe the effect of errors on the beam phase-space. in order to define tolerances and a global strategy for adjusting the SLC or an SLC-like system. We give this description at a qualitative level, and we give only orders of magnitude for the tolerances.

We first determine the evolution of the phase-space from the different kinds of errors. and in the different cases. We then analyse the effect of the distortions induced by these errors on the performance of the system - i.e. on the Iuminosity and on the backgrounds in the experimental apparatus, and the ability to correct them. As we shall see, this analysis enables to classify the different kinds of errors iu teras of their consequences and in terms of the type of correction they require (local or at the end) and to split up the tuning o: the machine sequentially into several pieces. This then allows to define criteria for setting tolerances, and to outline a global commissioning strategy.

This thought-process results from our accumulated experience in learning to operate the SLC. The actual conmissioning strategy which was followed differed initially from the prescription we give here. We briefly describe this initial strategy in the next rlapter.

Ideally. such conceptual analysis would be carried through at an early enough stage so as to be able to incorporate its most important implications into the basic design and architecture of the optics and of the control system.

\section{III.2 EFFECTS FROM IMPERFECTIONS}

\section{III.2.1 Errors in the Guide-Field and Betatron Oscillations}

Static dipole errors along the entire system cause spurious kicks to the beall, which then as a result executes betat ron oscillations in the quadrupole lattice. Such dipole errors arise trom misaligmments of the lenses in the focusing lattice, or from fied errors in the bending magnets of the transport. This is illustrated in F:n. 1.3a. The frequency of the betatron vicillation is referred to as the betatron frequency.

\section{III.2.2 Errors in Focusing-Field and Phase-Space Distortions}

III.3.1. Regular Focusing Field Errors and Betatron Wismateh 
Static quadrupole - or focusing - errors perturb the beam phase-space ellipse as shown in Fig. 14. Such focusing errors arise from gradient errors in the focusing lenses of the system. or, if the lattice uses sextupole magnets, from horizontal misalignments of the beam in these sextupoles. Because the quadrupole error will kick particles in this ellipse proportionally to their excursion in the quadrupole, its effect is the st ret ching of the ellipse shown in Fig. 14. The ellipse will then rotate in the coordinate system as it is imaged through the rest of the system. The bean envelope, or the projection of this ellipse on the thorizontal axis, will therefore beat along the optical array. Because the ellipse is invariant under ratation of $\pi$, the beating which is genetated has twice the betatron frequency. This is jllustrated in Fig. $13 \mathrm{~b}$.

\section{III.2.2 Skew Focusing Field Errors and Cross-Plane Coupling}

If sextupoles are used in the transport, as in the arcs and final focus sections, ur if the system does not satisfy mid-plane symmetry, such as the arcs, where the beam-line is rolled about the axis to allow following the terrain, then another type of focusing error can arise, which couples the horizontal and vertical planes. Such cross-plane coupling arises from vertical misalignments of the beam in the sextupoles, or from the imaging of beats in the beam envelope, generated by the regular focusing errors described above, across the rolls. In this case, the projections of the - coupled - four-dimensional phase-space onto the horizontal and vertical planes are in general not preserved and can result. if left tuncorrected. in larger effective emittances.

\section{III.3 EVOLUTION OF PERTURBATIONS - CORRECTIBILITY}

\section{III.3.1 Case of Chromaticity Corrected Optical Lattice}

lu parts of the system where the first order chromaticity of the lattice is corrected. such as the arcs or the final focus sections. the distortions of the envelope, from both regular and skew quadrupole errors, and the coherent oscillation of the beam generated by: dipole errors. are transmitted with the same frequency for all energies within the optical band-pass of the chromaticity-corrected optics. Lu this case. the effects of these errors can be corrected furlher downst ream in the system. This is in particular the case lor the arcs.

\section{III.3.2 Case of Lattice Not Corrected for Chromaticity}

In parts of the systen which do not have chomatically corrected optics, such as the 
linear accelerator, both these errors will gradually loose their coherence, due to the finte energy spread in the beam.

\section{III.3.2. I Incolrerent Propagation of Betatron Oscillations - Beam Dispersion}

The propagation of a dipole error will generate linear correlations between the entery and ti.e transverse coordinates. which we refer to as beam dispersion. After the phastshift accumulated between particles with maximum positive and negative energy errors has reached $\pi$. the effective phase-space area containing the beam will become as large as the phase-space $t$ ra jectory described by the betat ron oscillation induced by a dipole error. This occurs without violating the conservation of the six-dimensional $\left(x, x^{\prime}, y \cdot y^{\prime}, \frac{\delta E}{E}, z\right)$ phasespace volume. However this phase-space volume has become correlated, and its projection on one of the $\left(r, x^{\prime}\right)$ or $\left(y, y^{\prime}\right)$ planes cocresponds to an enlarged area. We illustrate this mechanism in Fig, 15a,b.

Such beam dispersion is correctable downstream, through adjustments in a purposely designed matching section.

\section{III.3.2.2 Incoherent Propagation of Betatron Mismatch - Chromatic Filarnentation}

Sinblarly: the propagation of a quadrupole error will cause second order correlations - or abercations - of energy to transverse coordinates. In this case, the puadrupole error generates an anomalous correlation between positions and angles in the transverse $\left(x, x^{\prime}\right)$ or $\left(y, y^{\prime}\right)$ phase-space. This correlated ellipse then rotates in phase-space with different speeds for different energy slices in the beam. as was described in II.1.3. Also here, when the accumulated phase-shift between slices with opposite and maximal energy error has reached $\pi$. the ensemble of trajectories composing the beam will be contained in an effective area as large as the circle in which the clistorted phase-ellipse in inscribed. The actual area orcupied by the trajectories is not enlarged, but acquires a complicated smeared st ructure. This effect, usually referred to as chromatic filannentation. is illust rated in Fig. 16.

In this case, the distortions cannot be corrected downst reant and untst be cortectert locally. This is particularly the case for the linear accelerator. and is important to numinize the eftertive entitance at the injection to the arcs.

\section{III.3.3 Frequency Analysis}

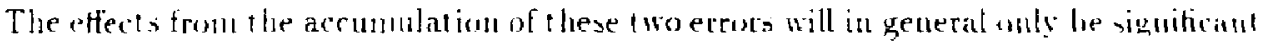
if thes add in phase. In the case of the dipule ertors. subsequent kicks of the same ign arul separated by 20 phase-adrance will add up and cause a bet at rou oscillation with increasing anplitude. In the care of the quadiupole errors, the beats in the benmenvehpe will arow 
if errors with the same sign are separated by $\pi$ phase-advance. In general. since the errors are randon, their Fourier component, at the betatron frequency for the dipole errors. and at twice the betatron frequency for the quadrupole errors, is not more significant statistically than other components in the frequency spectrum of the errors. Also. the specific distribution of the errors can be such that cumulative effects occur in one section of the array and not in the others.

We will examine the case of focusing errors in more detail.

\section{III.3.3.1 Average Growth from Random Forusing Errors}

In a repetitive FODO array, and for an ensemble of error distributions, the average fractional growth $\left\langle\frac{\Delta A}{i}\right\rangle$ of the beam phase-space which occurs at the end, in any of its dimensions, is related to the magnitude of the errors in the following way:

$$
\left\langle\frac{\Delta A}{A}\right\rangle=a^{\operatorname{lin} \sqrt{v}}
$$

Where $\epsilon_{R}$ is the standard deviation of the random errors, $N$ is the number of cells in the array, and $\lambda$ is a parameier or orter unity, which characterizes the lattice. For the arc laitice. we find througl simulation that $\lambda \simeq 2$. In this case, the exponential law arises because the differential growth of the bean size is proportional to the bean size and to the cell number for a quartrupole error at tuice the betatron frequency. The $\sqrt{F}$ in the exponent arises from the fact that the one standard deviation statistical expectation value for the magnitude of any given component in the spectrum of random quadrupole errors is $\epsilon_{R}: \bar{V}$.

Growth occuring through the accumulation of random errors can in some cases be co recled glabally, by deliberate introduction of a perturbation at the resonant frequency. Tlis is explored in more detail in sections VI. 3 and 4 for the case of locusing errors in the arc lattice.

When chromatic flamentation effects ate negligible. the induced sistortions remain coherent and can also be corrected downstream, in a purposely designed matching section. Stch ? matching section has been developed in the fual focus (see chapter VII)

\section{III.3.3.2 Bandwidth Limits from Discontinuties in the Focusing Lattice}

It is not uncommon that there be a rather large component in the spectrum of random errors at zero frepuency. from systematic errors in the calibration of the magnets or in the knowledge of the energy (if the lattice is not chromatically corrected). For a trily repertive lattice. such errors are liarmless and simply cause the betat ron frequency to be shitted.

This is however no longer the case if the lattice includes discontinuties, as is the case for the ares, where large rolls of up to ten degrees were introduced to enable folluwing 
the terrain (see section I1.1.5). These tolls were matched in pairs. to produce lorg-range cancellations of the distortions induced on the phase-space. This arrangeutent produced a relatively strong artificial sensitivity to systematic errors (see also section VI.2).

The distortions of the phase-s, ace ace correctable, either glubally. through systematic adjustments of the focusing elements, or further downstream, in the same conditions as in III.3.3.t.

\section{III.3.4 Electromagnetic Coupling to the Environment - Wake-Field Effects ${ }^{18}$}

Electromagnetic coupling of the beam charge distribution to the environment can result in correlations between transverse and longitudinal coordinates. Orbit excursions of the beam. caused by dipole errors, induce in general asymmetries in the image-currents on the inner walls of the vacuum chamber, or of any other structure seen by the beam. For large enough current, these asymmetries can result in significant transverse fields or wakefields - affecting the trailing part of the bunch, and thus causing a growth of the effec $\dagger$ ive enittance.

The most sensitive part of the system is the linear accelerator. where large wakefield effects are generated in the RF waveguide structure. for typical SLC beams. Beam trajectory errors must be corrected lorally and launch parameters must be stabilized at the entrance for minimization. Such wakefield effects, pertintent to the dynanics of the linear accelerator, are not considered furtler in this Thesis.

\section{III.3.5 Number of Free Parameters of Transverse Phase-Space}

It is important to know the number of independent phase-space distortions which can be corrected downst ream, in order to enable designing appropriate correction schemes. Wie will evaluate this number in the case of the transverse phase-space, and include also the four dispersion terms. We will include in our connting neither corcelations to the longitudinal coorlinate from wakefields, nor second order correlations bel ween energy and transverse cuordinates, from chromatic filamestation effects, both of which must be cortected locally.

\section{IIJ.3.5.1 Optical Transfer M/atrix}

The roptical tcansfer is described through a four by four matrix $R$ (trausport matrix), relating the four input coordinates $\vec{F}_{n}$ to the four output condinates $\vec{l}_{\text {uut }}$ :

$$
\vec{H}_{0,1}=R \cdot \dot{I}_{1,}
$$


Since the particle motions are governed by Hamilton's equations, this transfer matrix is constrained to be symplectic, which means that:

$$
R^{t} S R=S, \text { where } S=\left(\begin{array}{cccc}
0 & -1 & 0 & 0 \\
1 & 0 & 0 & 0 \\
0 & 0 & 0 & -1 \\
0 & 0 & 1 & 0
\end{array}\right)
$$

is the symplectic matrix in four dimensions. It can be shown ${ }^{19}$ that (12) results in six independent constraints on the matrix $R$, which has therefore a total of ten independant parameters.

\section{III.3.5.2 Bean Phase-Space Matrix}

The phase-space is described through a symmetrical four by four variance matrix $\sigma$, describing the correlations between the four $x, x^{\prime}, y$ and $y^{\prime}$ beam excursions. For a linear transformation of the coordinates described by $R$, this matrix is mapped through similarity transformation as follows:

$$
\sigma_{\mathrm{out}}=R \sigma_{\mathrm{in}} R^{t}
$$

\section{III.3.5.3 General Case of ['nequal Input Enittances}

The $\sigma$-matrix is fully specified through ten terms. However, only eight of these terms can be perturbed through a perturbation satisfying Hamilton's equations. This comes about because in general, Hamiltonian systems with two degrees of freedom can be shown to possess two canonical invariants ${ }^{20}$. Including the four linear dispersions, the phase-space can thus it general be perturbed in tuelve inclependent ways.

\section{III.3.5.+ Special Case of Equal Input Emittances}

In the case of equal input enittances, it is possible to show, ssing the symplectic constraint of (12), that two additiona! constraiuts exist on the bean phase-space, restricting the total aumber of independent distortions, including the four linear dispersions, to ten. The proof, which we outline below. is straightforward. What is lacking. however, is a physical understanding of the mechanism by which this symuetry bet ween the emittances can generate these two new constraints.

Proof. The proof consists of showing that because the transport matrix is symplectic, the $\sigma$-llatrix trandortued through this transport nutrix will also be synplectic. if the enuttances are equal in each platte at the input point. This then inposes two additional conditions on the elements of the $\sigma$.matrix. Consider an input phase-space with the two $\left(r, x^{\prime}\right)$ and $\left(y \cdot y^{\prime}\right)$ planes decoupled. The $\sigma$-natrix $\sigma a n$ representing this phase-space is block.diagonal. The entitunce in ench plane is given hy the square root of the determinant of each iwo lsy iwi 
black. Siace we assume that these two emitrances are equal, we scale fach two by two block hy bhis ronnnon value $c$ for the square root of the determinants. We can chus write;

$$
\frac{1}{\epsilon^{2}} \sigma_{i n} S \sigma_{i n}=S
$$

Next we multiply 114 ) by $R$ from the left and by $R^{t}$ (rom the right. and use (12). [n the cest of this proof, we will in additon set $\epsilon=1$. We have:

$$
R S R^{t}=R \sigma_{\text {in }} R^{t} S R \sigma_{i n} R^{t}
$$

In $(15)$, secognize $\sigma_{\text {out }}$ given by (13). We have thus:

$$
R S R^{\imath}=\sigma_{\text {out }} S \sigma_{\text {out }}
$$

To show that the output phase-space matrix $\sigma_{\text {aut }}$ is also symplectic, we need to show that the left hand siale of $(16)$ is equal to $S$. To do so, we start with $\{12)$ and take its inverse, go through the following thanipulations. and note that $S^{z}=-I$, where $I$ is the identity matrix. We have:

$$
\begin{gathered}
S^{-1}=R^{-1} S^{-1}\left(R^{t}\right)^{-1}, \\
R S^{-1} R^{t}=S^{-1}, \\
-R S R^{\prime}=-S .
\end{gathered}
$$

Thus the output $\sigma$-macrix $\sigma_{\text {aut }}$ is symplectie. Writing out the conditions explicitly at a point where positions and angles are simultaneously uncorrelated within each plane. as occurs for example nt the collision point, after A waist has been obtained there in each plane, i.e. where $\sigma_{12}=\sigma_{34}=0$, we obtaim:

$$
\left\{\begin{array}{l}
\sigma_{11} \sigma_{22}\left(1-r_{13}^{2}-r_{14}^{2}\right)=1 \\
\sigma_{33} \sigma_{44}=\sigma_{11} \sigma_{22} . \\
r_{23}=r_{14} \\
r_{24}=-r_{13} .
\end{array}\right.
$$

where we have defined the normalized correlations: $r_{y}=\sigma_{, j} / \sqrt{\sigma_{2,} \sigma_{\jmath}}$. Thits there is a tctal of Iutur constraints restricting the distortions of the phase-space if the input emittances in each of the two transverse plaues are eculal. In this case, there is a towal of ten independent distortions, including the four linear dispersiun terms.

\section{IfI.3.6 Classification of Phase-Space Pert urbations}

The different cases described in this section are summarized in Table 2. where we list. for each basic type of perturbation. the consequences on the bean phase-space if a correction is not applied until the very end, as a function of each location in the SLC: duwnstream of the damping rings. In each case, we then ask if the distortion is in principle correctable at the very end. 
Distortions correctable in principle may however not be tolerable in practice because of the requirement to minimize backgrounds in the experimental apparatus. As we shall explain in the next section, such requirements impose much more st ringent constraints on the distortions which can be allowed to accumulate to the very end. We do not attempt to incorporate such requirements in Table 2

For completeness, we have also included the emittince dilution effects from sinchrot ron radiation which occur in the arcs.

\section{III.4 CONSEQUENCES ON PERFORMANCE AND SENSITIVITIES}

\section{III.4.1 Sensitivities Pertinent to the Luminosity}

\section{III.4.1.1 Phase-Space Distortions}

The luminosity which can be obtained depends strongly on the ability to correct the distortions of the beam phase-space which we have described. The distortions which are uncorrectable at the very end, such as the correlations of transverse to longit udinal coordinates and chromatic filamentation effects, are translated into a larger effective enuttance. and affect the beam size directly through $(2)$ if they are not taken care of locally at their source. For the ten distortions which, in the case of equal emittances in both planes, can be corrected at the very end, the SLC' is equipect with a special matching section just downstrean of the ares. This matching section serves as a buffer section to absort the distortions. Because the beam size at the interaction point is produced by carefully bal. ancing higher order chromatic and geonetric aberrations, all of these ten distortions must be corrected to fully optinize the luninosity. Allowable distortions are of the order of one half to one standard deviations of the optimum parameters at the collision point, for reasonable luninosity to be maintained.

\section{[II. 4.1 .2 Beam Jitter}

Maintaining the luninosity also requires that the beams remain in cullision. The luminosity rechetion for an off:et corresponding to $q$ standard deviations of the beam can be calculated by perturbing the overlap integral performed to abtain (1) and (2). One sbtains. in the case of round beants:

$$
\mathcal{L}(q)=\mathcal{L}_{u} e^{*}+
$$

where $\dot{L}_{11}$ is the luminosity for an offset $q=0$. From ( 21$)$ can be seen that the maxinum offset between the two beams which can be tolecated is about $q \leq 1$ standard deviations. 
In the SLC, the main source of pulse to pulse jitter in the beam position resides in the kicker magnets used to extract the bezms from the damping rings. This pulse to pulse jitter causes variations in the beam trajectory along the whole nachine, and as mentionned in III.3.5, such variation can blow up the emittance through transverse wake-field effects. Therefore, at high intensity, the tolerance to beam position jitter is in fact not set by the requirement to maintain the beans in collision, but rather by the requirement to mininize these wakefield effects. With large local energy spread (several percent.) introduced on purpose along the bunch to reduce the build-up of wake-field tails (so-called BNS Damping), the tolerance to launch jitter is of the order of a tenth of a. standard deviation of the transverse beam distributions, at $510^{10}$ particles per bunch.

\section{III.4.1.3 Intensity Dependance}

SIore generally. the entire system enters a new regime at high intensity. Because of the wake-field effects, tolerances for maintaining a nominal phase-space at the end of the linear accelerator become more stringent with increasing intensity. It is therefore expected that the effective enuitance at the collision point will be a growing function of the beam intensity, and that the gain in the luminosity from raising the current will not be as rapid as that implied through (1).

\section{III.4.2 Sensitivities Pertinent to Backgrounds in the Experimental Apparatus}

\section{III.4.2.1 Introduction}

Beyond the goal of maximizing the lumiuosity, the beam phase-space must also be stabilized to unimize backgrounds in the experimental apparat us. Such backgrounds arise wheti tails of the bean generated by wrkefields in the linear accelerator strike apertures near the interaction point, causing electromagnetic stowers into the detector, or further upstrean in the final focus beam-line. where they produce mutus which can reach the detectur.

Such beam tails are clipped by using collinators at the exit of the linac and in the arcs. Icleally, such collination would be perfect and define a full-proof beam stay-ciear. alluwing to precisely shadow the critical apertures in the final focus.

\section{1.2.2 Principle of Collimation}

The basic principle of a cullimation sytem is illustrated in Fig. 1 $\vec{r}$. as an example. Sinulisticalls. a minimum of thee collinuators is used to enclose a three-dimensional $\left\{I^{\prime} . \frac{J E}{E}\right.$ J phase-space volume in the horizontal plane. The two first ones are upitream of a bending magnet, and serve mostly to define the spatial and angular dimensions. "The" 
third one is downstream. It serves mainly to define the energy.

If the two spatial and angular dimensions of this volume are defined at reference point (0) by $X(0)$ and $X^{\prime}(0)$, and if the desired cut on the energy is $\frac{2 E}{E}$. then the settings of the collimators is obtained by inverting the three linear equations relating the parameters of this rolume at the reference point to the spatial coordinates $X(1), X(2)$ and $I(3)$ of the beam at each collimator. If $R^{(i)}$, for $i=1,2$ and 3 , represent the optical transfer mat rices between the reference point and the three collimators, then these three relations can be writen:

$$
X(i)=R_{11}^{(1)} Y(0)+R_{1 !}^{(1)} X^{\prime}(0)+R_{16}^{(1)} \frac{\Delta E}{E} .
$$

Similar equations can be writen for the vertical plane.

In order that the equations in (22) be far from degeneracy, it is important to choose the locations of the two first collimators with about a $\pi / 2$ betation phase-advance separation. and the third collinator where the natural dispersive size of ihe beam is significantly larger than its betatron size. Moreover, with high current small emittance beams, it is desirable to collimate the beams at relatjvely high values of the 3 -parameter, so as to minimize the power deposition on the collinators".

In many cases, a more sophisticated collimation system is usually required, of which the arrangement described above is only the first stage. There are two basic reasons tor this. The first is that the equations in (22) fully define the specified phase-space volume only to the extent that the optics between them is independent of energy over the specified $=\frac{\partial E}{E}$ range. To minimize such chromatic effects it is important to place the collimators as close to each other as possible. The second perhaps more important reason is that each collimator inpinged by the beam will reradiate some flaction of the incident beam through edge-scattering. For this reason, it is often desirable to add a fourth collimator $\pi / 2$ phase-advance upstream of the first one in Fig. 17. With this redundant collinator. the two following ones can also serve to clean up large angle debris. and the last one to catch components of this debris witl large energy errors.

Even with this extra collimator, the scheme we have described will not enable to entirely suppress particles from forbidten regions beyond the specified bean-stay-clear. and there will be a certain probability for transmitting particles into such regions. This probability can be reduced by adding more stages to the collination system.

\section{[II.4.2.3 Beanu-Stay-C'Jear Requirements}

In relation to the nonumal bean size, the tighest aperture in the system are the quatrupoles near the collision point. Although these qualrupoles only lepresent une be. tatrm phase. it is appropriate to define a full phase-space volume comtesponding lo thej

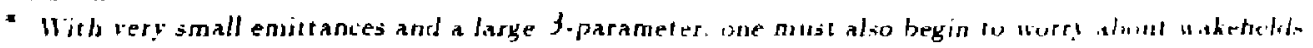
frum the discuntunuties in the beam-pipe genernted by tha collimaturs 
aperture. The reason is that the phase relation between the collimation and these apertures is not well clefined for typical tail particles with large energy errors. if the collinators are far upstream in the system. For nominal enittance and with the optics in the final focus adjusted to minimize the beam size at the collision point, this aperture. and theretore the phase-space volume to be defined by the collimators, corresponds to about five standard deviations of the bean phase-space distribution*.

\section{III.4.2.4 Constraints on Performance from Partial Collimation}

\section{(1) Recingrements on Beam Stability}

During initial conmissioning, the collimation scheme avajlable at the SLC was insufficient. Only a primary cut was being made, and, in addition, the different dimensions of phase-space were cut non-orthogonally and sometimes a significart betatron pliase-distance apart. As a result, not only could secondaries be transmitted, but also primary particles from corners of phase-space which are significantly correlated. C'onsequently, the bean stay-clear defined by these collinators was far from [ull-proof and was very sensitive to the detailed shape of the phase-space at the end of the linear accelerator. This generated an abnormally stringent tolerance on the stability of the phase-space. Small variatiuns would c.ten cause the collimator configuration to no longer be optimal and backgrounds to rise in the experimental apparatus.

With ideal collimation, the end of the linear accelerator would provide a ireak-point in the accumulation of errors, separating the minimization of backgrounds produced by the errors in the sections upstream of the arcs from tuning in the arcs and final focus to maximuze or simply maintain the huthinosity. The fraction of particles outside of the beam siay-clear defined by the collimators would then be small enough not to generate backgrounds. jutlependent of sariations in the beam plase-space upstream of this breakpoint. Variations would of course still need to be stabilized within this (five standard deviation beam stay-clear, in order to maint ain the luminosity. its we clescribed in III.t.1, such stabilization must be done roughly at the level of one half to nne standard deviations.

Shori of such a well defined break-point, the tolerance to changes in the beam shape at the end of the linear accelerator is very tight. In the initial commissioning of the system. it was of the urder of a tenth of a standard deviation, which was very hard to maintain. The collimation scheme is at present heing upgraded with additimal collimators. This is expected to aituce this sensitivity substantially.

This cumes atout from the fact that for the optimum 3- of about $0.5 \mathrm{~cm}$ required at the interaition point to optimize the lummosity. the one standard teriation balue of the beam size in the last lensos. reaches a maxmum of abuut three milumeters, and from the fact that the aperrure of ihese last lenses ls: alwuc rwo tentimeters. Thus. since one must allow for offecentering from nisteering uf about une standatal clevatian. the dseful aperture is about seventera millimeters or just over tive stamiard dewarmas of the beaula size 
(2) Requicement to Operate with Detuned Optics

Operationally, in order to reduce the requirement for a good full-proof five standard deviation cut on the phase-space, the optical demagnification in the final focus is reduced by about a factor two. This reduces the natural beam size in all the high- 3 quadrupoles near the interaction point by a factor two, and lowers the probability that stray particles which have been transmitted through the collimation at the end of the linear accelerator. wander outside of the beam stay-clear specified by the aperture. This is however costly in overall perfornance since the beam size at the collision point is thereby enlarged. and thus the luminosity reduced.

(3) Requirements on Phase-Space at the Arc Exit

The sensitivity to insufficiently collinated beam-tails was in addition enhanced, inirially because the apertures of the final focus beam-line, upstream of the correction elements used as part of the optical buffer section for phase-space distortions (described in [II.4.11, are tighter than those in the arcs, by factors of five to ten, after normalising to the nominal bean size. For this reason, otherwise correctable phase-space distortions could cause scraping off this aperture. This in turn would generate muons which could reach the detector. The tolerance to beam loss along the final focus beam-line was of the order of $10^{\circ}$ particles per crossings, or one part per thousand of a beam with $10^{10}$ particles per bunch. This was a very st ringent tolerance, which required that the beam phase-space be nearly matched at the arc exit. To meet this tolerance special optical controls within the arc lattice had to be designed. to enable controlling the beam size and shape at the end. More recently. special magnetic shielding has in addition been installed in the final focus tunuel. to relax tolerance to bean loss there by almost an order of magnitude.

\section{III.5 TOLERANCES AND TUNING: THREE MAJOR PIECES}

\section{III.5.1 Introduction}

It is appropriate to esaluate lulerances to errors and a global strategy for adjusting t! r youn by dividing up the SLC in three major picces. and in terms of their orerall efferi on pelformance. described in IIl 4.

Ther ihree majol piectsiare:

1. The pasts of the SLC which ace upsteatu of the damping rings: the elect lon aud

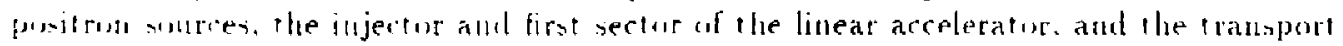


lines feeding the beams into the damping rings:

2. The parts of the SLC! which are between the damping rings and the end of the linear accelerator: the transport lines connecting the damping rings to the the linear accolerator. and the linear accelerator,

3. And the parts of the SLC which are between the end of the linear accelerator and the collision point: the arcs and final focus sections.

\section{III.5.2 Sources and Injector}

The damping rings. thanks to the radiation danping, will "standardize" virtually anything* that is injected into them. Therefore, errors in the sections upstrean of the damping rings matter only if the distortions generated beconte larger than the available aperture, in which case the transmission into the rings is derated. The detailed shape of the beam and its emittance are not very important as long as the beans niakes it into the rings without getting scraped off. The tolerances are 1 'erefore relatively loose on the transport, as long as the phase-space generated at the soicte is not athnormally la:ge. This is true both of static and dynamic errors. In this way, except for possityle losses in beam cutrent, errors and tuning upstream and downstream of the damping rings are independent of each-other. The damping rings provide a breakpoint in the overall accumulation of errors, where the beam "looses memory" of what perturbed it before.

\section{III.5.3 Ring to Linear Accelerator Section and Linear Accelerator}

Errors in the ring to linear accelerator section and in the linear accelerator will cause a combination of effective emitrance growt band bet at ron mismatch. The effective enitt ance

* This is strictly true only for a storage time lage cumpared with the damping rime. If the storaye time. which is equal to the time between pulses in the linear accelerator, is $T$. and the dampiny time is $T$. then output emittance ian be uritter

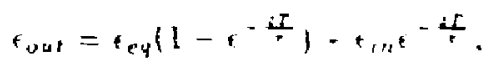

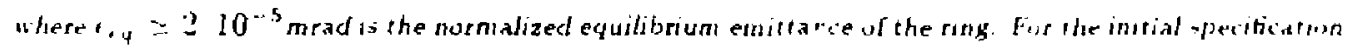
if the sLC at $120 \mathrm{~Hz}, T=3.3 \mathrm{~ms}$. The ramping time is $T=3 \mathrm{~ms}$. This alluws to talculate ptre

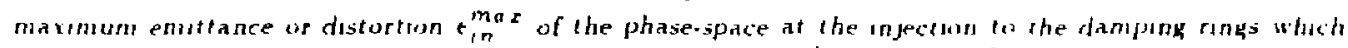

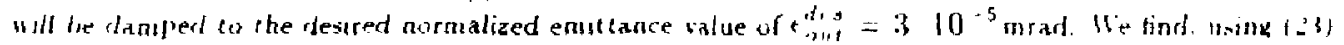

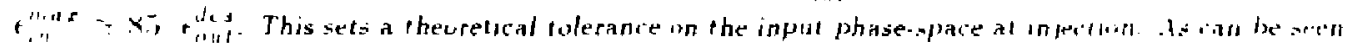

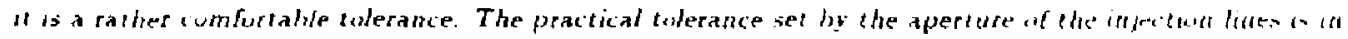
fact mure iringent 
growth is uncorrectable and affects the luminosity that can be achieved dicectly. The mismatch of the phase-space will if left uncorrected combine with the mismatch generated in the arcs and fual focus. As long as such errors are static, 1 his is not a major problem for the luminosity. The effects from, and sensitivity to such mismatch. from the point of view of the luminosity, was described in [1].4.1.

The static tolerances must be specified to avoid significant emitrance growth. If the collimation system at the end of the linear accelerator is close to perfect, and defines a fu!lproof beam-stay-clear, as described in I!1.4.2. then these tolerances must be determined to maintain a stable pliase-space at the end of the accelerator to one half tc one standard deviations of the nominal parameters. In this case, the tolerances in the linear arcelerator are of the order of $100 \mu \mathrm{m}$ for the orbit errors, and of the order of half a percent for the focusing errors, in order to assure emittance growth below a factor 1.5 ". The tolerance on the focusing errors is not difficult to meet for the quadrupole strengths. It is however quite difficult to know the energy of the gradually accelerated bean. locaily, to that precision.

In addition, because this energy depends on the population of klystrous used to generati the RF power which is fed to the beam, and on the correct phasing of this RF at each station, and because both these are not entjrely stabilized, the phase-space mismatch and effective enuttance growth are not necessarily stable. It is therefore most often unreasonable to allow them to accumulate and combine with resiclual ercors in the arcs and final focus, because as we will see, the tuming procedures for the final focus are complex and lack orthogonality, which means that a full reoptimization can be reyuired even for modest changes. In general, this second part of the system can be considered the "active" part of the SLC. Stabilization is still an ongoing effort.

In the case of the ring to linear accelerator transport line, a chromaticity corrected optical system. the tolecances are determined by tie requirement to minimize higher order abertations. This is similar to the requirement: in the final forus which we describe in chapter VII. Work on this is also ongoing.

\section{III.5.4 Arcs and Final Focus: Range of Optical Buffer Section}

Because the arc lattice is achrontatic, the errors are transmulted coherently io the ench, and can therefore be corrected opticaly in the fual focns. The final focus is equipped with special optica! uatching elements to pertorm sucit corrections. Because the SLC has nomually equal emitiances in both transterse planes. this optical matching is rlesigned to correct the ten possible independent phase-space distortions, and uses therefore ten variable elements. The correction range allows to match plase-upaces distorted by as much as four standard eleviations of the nominal paraneters. and in any uf its dimensins.

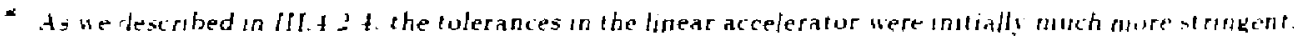
riue to the fact that the collination system was insufficont 
If the beam phase-space is close to matclied at the exit of the linear accelerator, and if for a momeut we do not consider the background problems, then the tule rances in errors in the arcs are in principle set by the correction range available in the final focus, We can then estimate the tolerances through (10). by calculating the standard deviation ${ }_{R} R$ of the random errors compatible with the factor four correction range. We find, since the number of cells is $V=230$, and if require a $98 \%$ confidence level: $t_{A}=0.023$.

This is a rather loose tolerance. Initially the system was uuch nuore sennitive. for the two following reasons.

1. As already mentionned in III.3.3.2, the discontinuities introduced into the are lattice by the rolls of the achronlats resulted before the modification of the rull transitions in a severe tolerance to systematic errors, of the order of 0.005 (see section VI.2)

2. As already mentionned in III.4.2.4, the smaller normalized apertures in the finat locus beam-line required initially that the beam phase-space be negligeably mismatched at the sid of the arc. The tolerances to random errors were in this case also of the order of $\epsilon_{R}=0.005$.

\section{III.5.5 Global Tuning Strategy}

The above considerations on the tolerances and on the tuning in the SLC' are summarized in Fig. 18. Both static and dynamic random errurs naturally cause an average growth of the phase-space with the exponential of the square root of the distance. Two breakpoints exist. where the beam in in principle reset to its nominal condition. Tliese breakpoint separate the tuning in the three parts of the SLC which they define. The first breakpoint. the damping rings, re-standardizes the bean perfectly. The secoml lreakprint. consisting of the colfinators at the end of the linear accelerator. serves to separate lunith related to maximizing the luminosity from the recluirement to minimize hackuromuls in the experimental apparatus. The settings of the collimaturs are leternumed by the optical set-up arrived at in the arcs and hinal forus. This set-np results from tuning buth these sections to maximize the luminosity at the interaction print. After this. he second break point - the collimators - decouples the backgrounds in the detector from thuing in the upstreau sections. The tolerable etror level and performane of feedhack iorrections and tuning are retermined at earh stage by the "capture-range" defined by "ach of these breakpo ..ts.

Much of the present challenge in optimizing the overall tumne st ratcoge if the 4.6 is the detinition of the second breakpoint. by improving the collianat ion it the 'mol of the

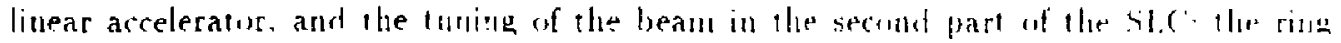
to linear accelerator and linear accelerator sections. Once this i donte. the uplow in the

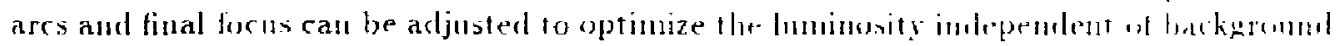
consideral ions. 
IV. SLC TURN-ON PROGRAM 


\section{IV.I INTRODUCTION}

What was destribed above is the current status and understanding of the commissioning of the SLC. Both result trom our accumulated experience with this machine ${ }^{2}$. The initial program proceeded in a rather different way, as we were learning to operate this new kind of ulachine.

Commissioning and tests of successive stages of the SLC were an ongoing enterprise since the fall of 1981 , and throughout the construction period (fall 1984 to spring $198 i$ ). Subsequently, and for a lit the over one year, much dedicated work was devoted to the newly installed arcs and final focus sections, with continuing improvements in the upstream parts. This last half-year, the full extent of the overall tuning problens of the SLC have been recognized. Special efforts are now dedicated to optimize each system according to the general framework described in III.5.5.

\section{IV.2 EARLY COMMISSIONING PHILOSOPHY}

The early commissioning philosophy for the systems downstream of the clamping rings consisted in transmitring the beam rapidly and as far as possible into each newly built and installed systens to enable early diagnosis of major installation and coustruction ecrors. After this was done, the program continued with an overall tuning and correction strategy which treated the final forus section as an optical buffer section for the accumulated errors in the whole machine.

The program therefore began early on trying to focus the beam to a small size at the collision point. although with little success. because of primarily two reasons: the sperific problens with the are laltice. which were not understoud intially and were discovered experintentally, and the lack of understanding and stability of the phase-space at the end of the linear accelerator, die to insulficient time spert commissioning the upst ream parts of the til.c.

\section{IV.3 OPTIMIZING THE ARC PHASE-ADVANCE ${ }^{2.3}$}

Afrer the nuechanisu which cansed the are lattice a chistort the bean phace-space

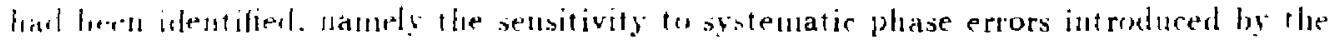


rolls. the program shifted towards minimizing the distortions, through a combination of ineasurements of the phase-advance in each of the achromats. and partial corrections. possible in the combined function magnets, by physically moving magnets and by conibining trim windings in each achromat and a global imbalance between locusing and defocusing magnets set up in a separate circuit.

\section{IV.4 MODIFICATION OF ROLL-BOUNDARIES}

A second cure which was devised consisted in modifying the roll distributions to generate transitions with a greater tolerance to systematic phase errors. The adjust ments of the arc phase-zdvance had brought the system close to specification, and liad minimized the coupling to the point where it could be hantled relatively well by the opticat matching sections in the final focus. It was however felt important for future operability to implement this passive cure, which made the system significantly more error-tolerant, particularly for equal or close to equal input emittances. After implementation of this modification, the ares behaved much as arcs without rolls, and although some cross-plane coupling rmained at the arc exit, the beam envelope was near its nominal size. This, and improvements macle in controlling the phase-space at the eud of the linear accelerator, enabled to obtain and to maintain snall focused spots of about $5 \mu \mathrm{m}$ at the collision point.

\section{IV.5 NEW AND TIGHTER CONSTRAINTS FROM BACKGROUNDS}

In the next conmissioning phase. the problems of mininizing backgrounds in the detector began to be included in the tuning strategy. The initial collimation strategy concentrated the primary collimation in the final forus. where nearly every accessible place un the beam-line is at the same hetatron phase because of the large J-functions, and where only sniall fractions of the phase-space conld be cut without generating excessive ummbers of tumous teaching the cletectnr. Becaitse of these t wo problemis. a nearly mat ched pliase-space was fund to be required at the ebtance to the final focus. For this reason. two arditional modifications had to be introduced. Firstly the collimation st mategy had (1) be modified. and the primary collimation resleployed to the linear accelelator to arc bean line ("bean switchyard") and to the art "reserse bend". Secondly. a new scheme tior dosing optical corrections with the combined funclion are nagnels. Jurungh harmonic

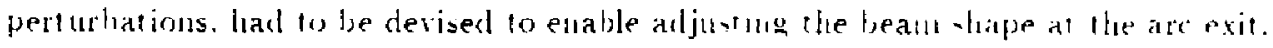

\section{6 COLLIDING BEAM OPERATION}


After this, the program proceeded trying to maintain stable colliding heams at the interaction point, with low backgrounds. This involved both small adjustments 10 the optics in the arcs and final focus and extensive use of the tuew collimators. In this phase. the beams were made to collide and maintained in collision for the first tine by letecting beam-beam deflections, which result if the beams don't collide head-on.

The beam-beam deflection method was proposed at an early stage in the commissioning. as a way to bring the focused micron size beans into collision at the interaction point. using the electromagnetic fields produced by the charged electron and positron bunches. These fields are intense enough to deflect the opposing bunch coherently, if the collision is not head-on. The deflections can be measured with strip-line beam position monitors at the system's high $\beta$-points, where they are magnified.

This method is one of the three obser able effects from the beam-beam interaction at the SLC. The two other methods are the synchrotron radiation emitted as the particles are deflected in the electromagnetic field of the opposing bunch ("beamstrahlung"), and the enlargement of the betatron angular spread at the interaction point from these same fields ("disruption").

With its strong and relatively easy to detect signals, the beam-beam deflection methorl has become the primary method for maintaining the beams ill collision. It also provides a signal st rongly correlated with the luninosity, which may in the future be used to optiunize the beam sizes of the two beans at the interaction point.

\section{IV.7 TOO MANY KNOBS}

With a not fully stabilized phase-space at the end of the linear accelerator. with the presence of still some unstable pieces of hardware in the ares and final focus, and because of insufficient and solely primary collimation, the program often became non-convergent: collimators were set-up one day for a given phase-space; then the bean plase-space would change and particles would begin to get transmitted into forbiditen parts of the required beam stay-clear. The response uould be a combination of re-adjustments of the collimators and of the optics in the arcs and final focus, which images the collimators. This wuld then require further optical adjustmuents to re-optimize the beam size at the interactiun point and so on. It was understood that to sulte this problem. the progran had fo, begin by ancertaining a stable optical set-mp in the arcs and tinal focus. Where currections resuld not lue nate con intally to feedback on rariations in the heam sizes at the collision

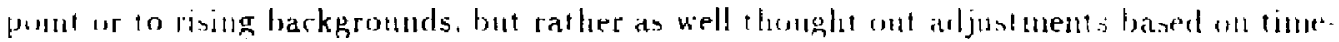

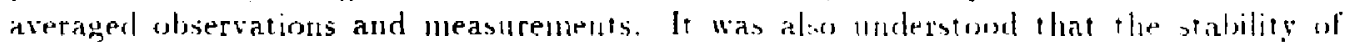
the linear accelerator is essential, and that a more fitl-prest collimation system is decelerl

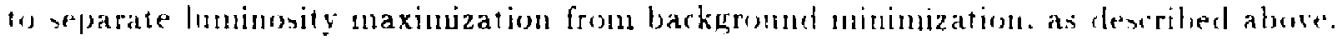


Also, reliability issues began to receive more attention. as the time to reach a well optinuzed set-up became longer, with the ever increasing requirements put on the phase-space. and sometimes alnost reached the time between hardware failures in the marhine.

\section{IV.8 PRESENT GOALS}

The SLC is at present being restarted. A new collination system is to be installed at the end of the linear arcelerator. Also, special magnetic shielding has been installed in the final forus tunnel, to reduce the rate of muons generated by beam tails getting scrapped off the vacuum chamber of the early parts of the final focus. This, combined with the already existing slits. will enable beam tails to be cut more efficiently.

Furthermore, much attention is now given to controlling and stabilizing the beam phase-space at the end of the linear accelerator. This, coupled with iniprovements in handling the backgrounds, is expected to enable using the nominal rather than the det uned optical configuration in the final focus, and thus to reach a lower $3^{*}$-parameter for the beam at the interaction point.

In addition, tests are being made with larger intensities ( 2 and $310^{10}$ particles per bunch), to begin exploring experimentally the hich current dynamics and the possibility to reduce wakefield effects through the introduction of a large energy spread along the bunch in the early sectors of the linear accelerator (so-called BNS damping).

The combination of these improvements, and of others not nentionned here, is expected to enable producing a useful rate of detectable $Z^{\circ}$ particles. 


\section{OVERVIEW OF OPTICAL TUNING IN THE ARCS AND FINAL FOCUS}




\section{V.1 INTRODUCTION}

The optical tuning procedures which were devised to adjust the arcs and final focus sections of the SLC result from:

1. The basic principles of the optical design of these two sections, which were described in chapter II, and

2. The global evaluation of the sensitivity to imperfections and of the overall tuming considerations described in chapter III.

These procedure where furthermore developed in the framework of the actual strategy followed to commission the SLC, as sketched in chapter IV.

\section{V.2 OPTICAI, TUNING IN THE ARCS}

The SLC ares have an achromatic lattice consisting of a FODO array. To maximize the packing factor. combined function magnets are used. All magnets except in the "beam switchyard" and "reverse bend" are on the same power supply. The magnets are equipped with backleg windings, on a achromat by achromat basis. These backleg windings were originally introduced to provide a gradual adaptation of the magnet excitation to the bean energy, which decreases by about $1 \mathrm{GeV}$ because of the synchrotron radiation enitted in the bending field. In addition. an imbalance belween focusing and defocusing magnets can be set up globally over the length of the arc, through a separate circuit.

The goal of the tuning in the arss is primarily to render the optical transfer as close as possible to an identity transformation. To some extent, tuning in the arcs is also used to supplentent the tuning of the final focus, as expiained in III.4.2.4. in order to correct for erors in the incoming phase-space. Three nethods were clevised for this purpose. 'The two first mes are specifically geared towards optimizing the lattice in the presence of the large discontuities introduced by the rolls. The third one is a more general method for ardjustug long repetitive FODO artays.

\section{V.2.1 Phase-Adjust ments ${ }^{3}$}

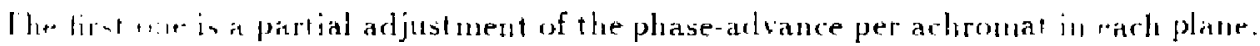

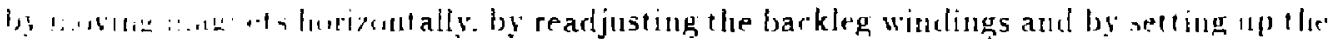


global imbalance. This had to be done since the rolls were in the original clesign grouped in compensating pairs separated by one or several achromats, to adjust the phase-advance in each plane near its design value of $0 \pi$ per achromat.

\section{V.2.2 Smoothing out Roll Discontinuities}

The second one is a modification to the roll distribution, enabling a smoother transition, which was shown to reduce the sensitivity of the lattice to systematic errors substan:tially. This was a passive correction. which reduced the need for doing precise adjust ments of the phase-advance.

This modification is clescribed in section Vl.2.

\section{V.2.3 Harmonic Corrections}

The third correction is a harmonic correction. It applies more generally to FODO arrays, and allows to correct any kind of error generated in the arcs. It is designed in particular to correct for coherent build-up of the phase-space clistortions due to errors at twice the belatron frequency. including both in-plane phase-space distortions and crossplane coupling. The required trim corrections were introduced in the conbined function unagnets by rewiring the backleg windings in each magnet of the last seven achronats of each arc into sets. spatially modulated at twice the betatron frequency. By driving the upper and lower windings of each magnet separately, it is possible to generate both regular and skew quadrupole perturbations in the magnets. In total, the lattice san be perturbed in nine itclependent ways through this method. These additional adjustments allow both to take out errors in the lattice and to correct disiortions of the phase-space injected into the arcs.

This nethod is clescribed in sections YI.3 and 4.

\section{V.3 OPTICAL TUNIYG IN THE FINAL FOCLS}

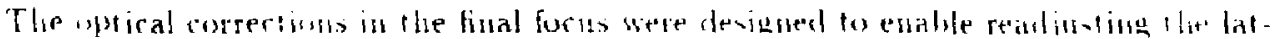

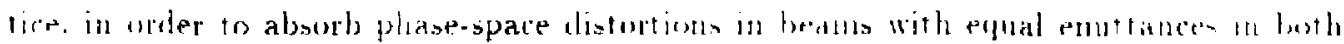
plances at the injection to the section. The design of these adjustments and el the s antegy

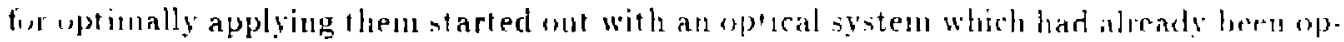

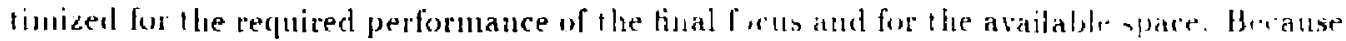


of this. the design work had to take a pragmatic approach, and only slight modifications to the lattice were possible. As a result, some of the correction modules straddle several of the telescopes of the final focus, and are therefore strongly coupled. In uddition, one of them has a practical range severely limited by perturbations to the trajectory produced on the outgoing spent bean.

The maximum adjustments of the optirs which are possible enable absorbing distortions of up to a factor four in any of the orientstiuns of the plase-space. All components of the transverse phase-space and the couplings between them must be controlled to minimize the beam size at the collision poir:- This involves ten independent distortions of the phase-space, and thus the correction algorithm uses ten variable quadrupoles.

The adjustments are grouped in three sets:

1. Four corrections to minimize the spatial and angular dispersion in both planes. using two pairs of quadrupoles to perturb the matching of the clispersion in the first section of the final focus. The pairs are separated by $\frac{\pi}{2}$ and consist of regular and skew quadrupole for control in each plane.

2. Three corrections to the betatron angular spread at the interaction point, by controlting the nagnitude of $\left\langle x^{\prime 2}\right\rangle$ and of $\left\langle y^{\prime 2}\right\rangle$, and by minimizing the $\left\langle x^{\prime} y^{\prime}\right\rangle$ correlation. This is done using the two last erect quadrupoles and a skew quadrupole in the upper telescope, where the demagnification of the final focus is initiated.

3. Three adjustments to position the waists in both planes at the interaction point, by tninimizing the correlations between the positions $x, y$ and the angles $x^{\prime}, y^{\prime}$ in both planes. This is done using trim windings on the next to last two quadrupoles of the final telescupe. and with a skew quadrupole just upstream.

The ten variable quadrupoles used for these corrections are shown in Fig. 12. Because each correction is coupled to the ones downstream, they must be applied sequentially. A flow diagram illustrating this sequential application is shown in Fig. 19.

The seven first adjustments are st congly coupled and depend non-linearly on plasespace parameters. The final three waist corrections can be orthogonalized independent of the input phase-space. A ten-dinensional non-linear fitting program has been dereloped to match the latcice in the final focus to the input bean. Local orthogonal "knobs" are alsn rlefined for tine-tweaking around the initial solution, although this is not always practical becallse of steering from the lenses.

The nprical tuming of the final fusus is the subject of chapter VIt. 


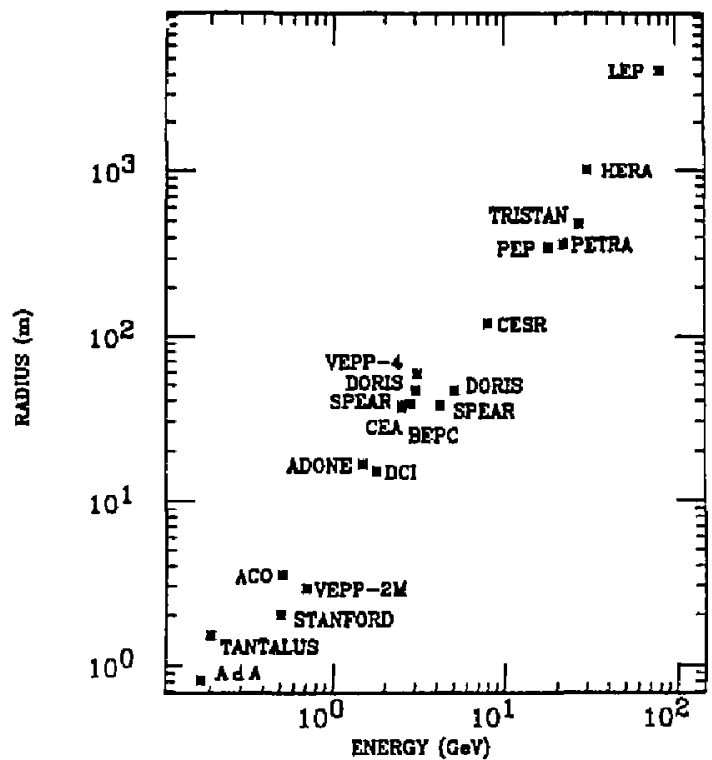

Figure 1. A verage radius of the majority of the storage rings as a function of their energy. The dependance is close to quadratic.

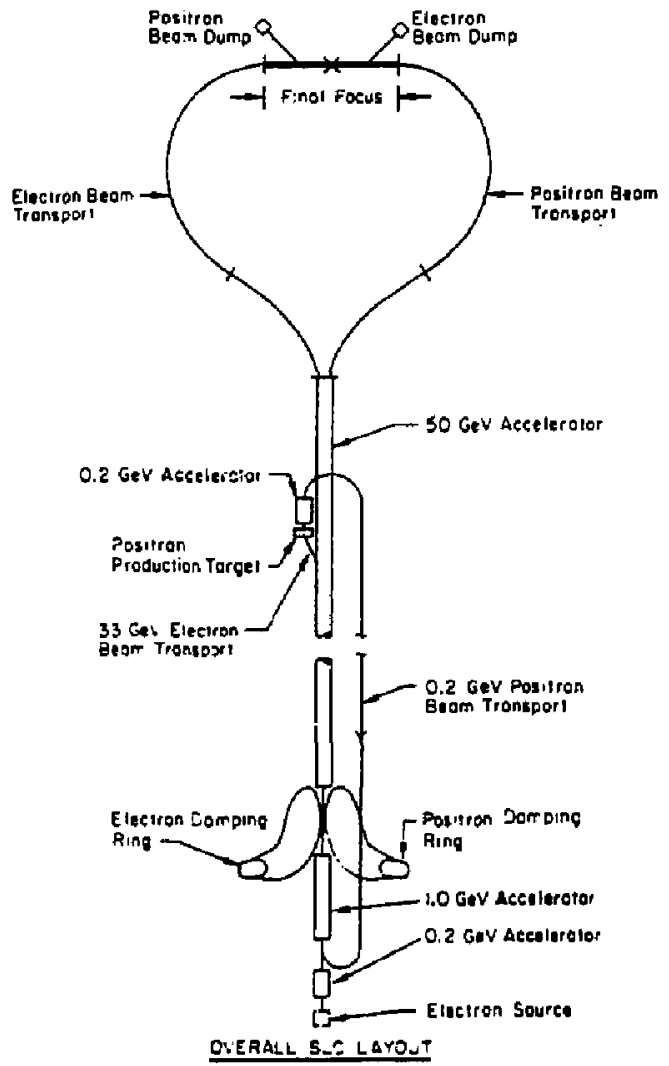

Figure 2. The Stanford Linear Collider. 


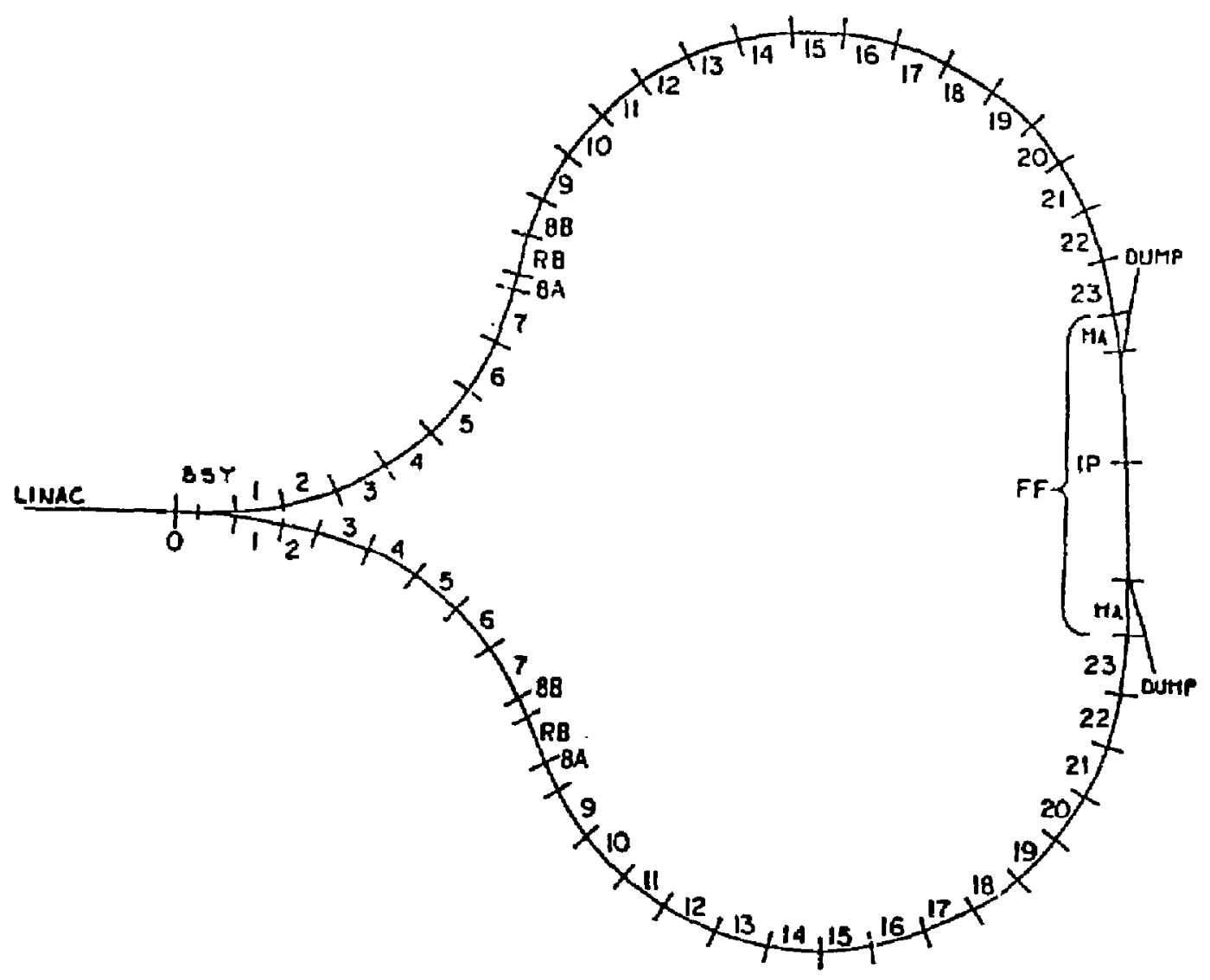

Figure 3. General layout of the arcs. The numbers refer to each achromat.
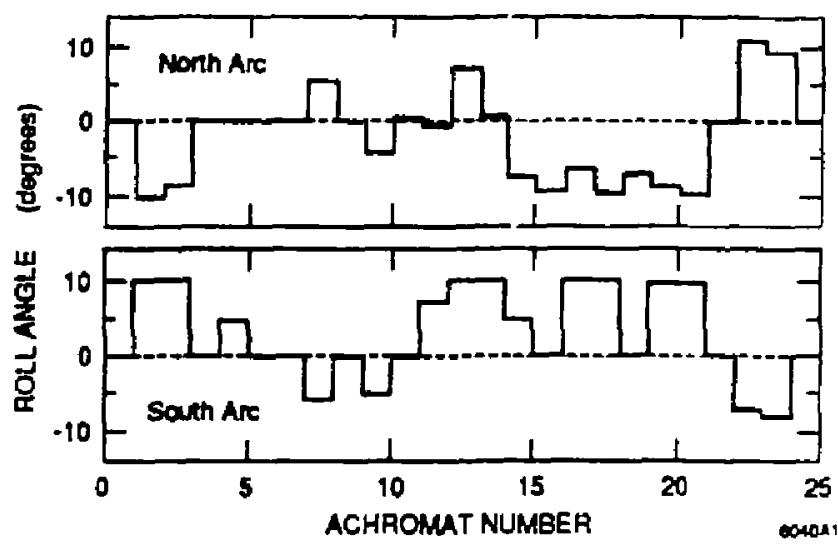

Figure 5. Rolls about axis of achromats in north and south arcs. 

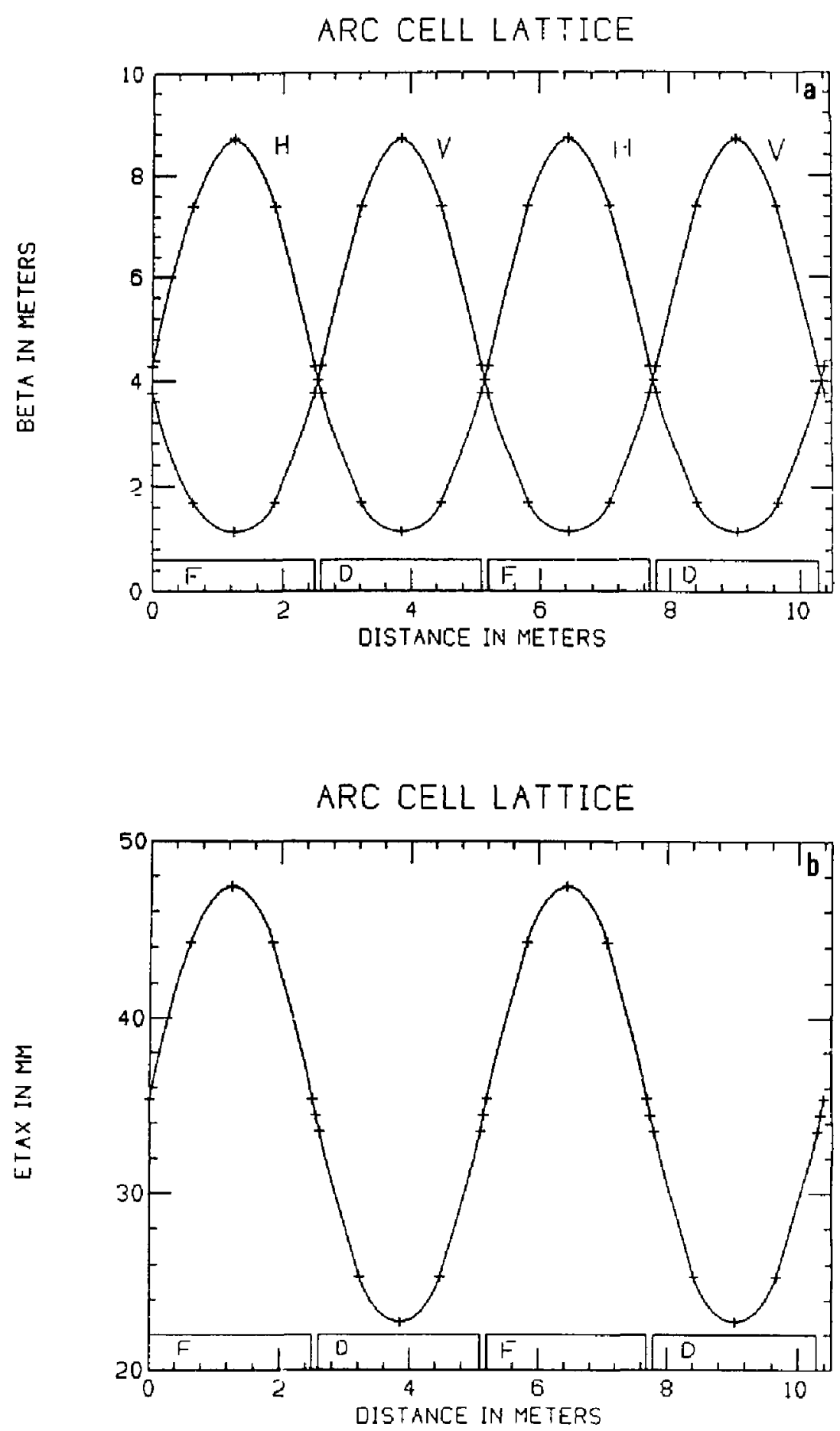

Figure 4. Optical functions of the arc lattjce: in (a) the $\beta$-function, and in (b) the $\eta$-function. 


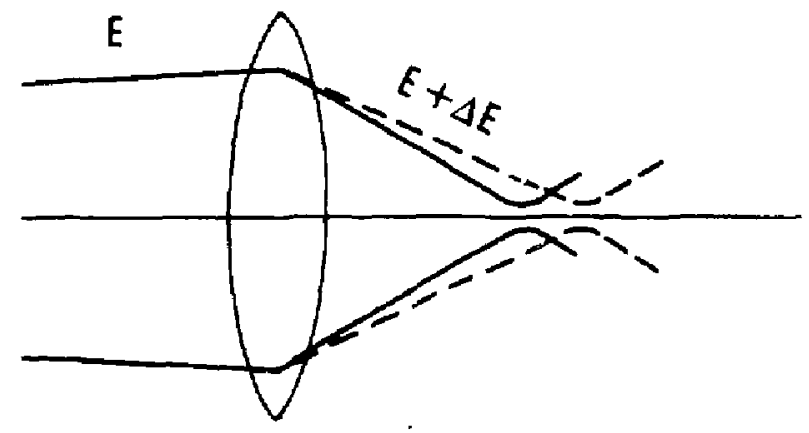

Figure 6. Longitudinal displacement of the focal point with the energy.

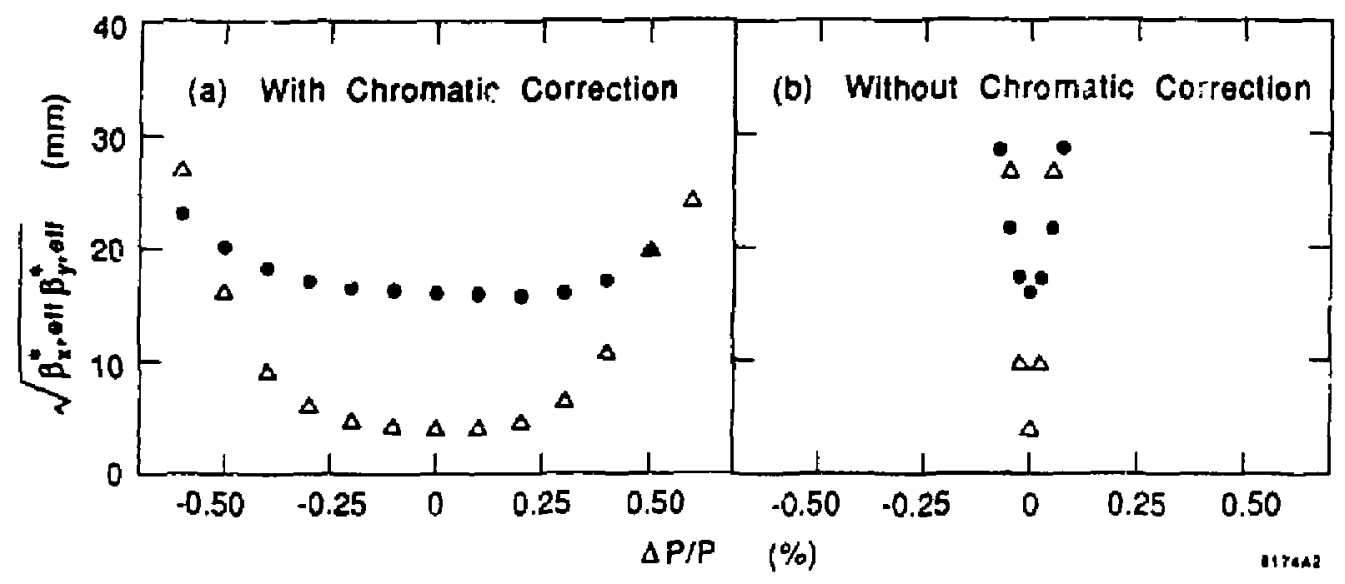

Figure 7. Variation of the $\beta$-parameler with the fractional energy error in the final focus section, with chromaticity correction (a), and without chromatjcity correction (b). As can be seen, the optical bandpass of the system is enlarged by the chromaticity correction. 


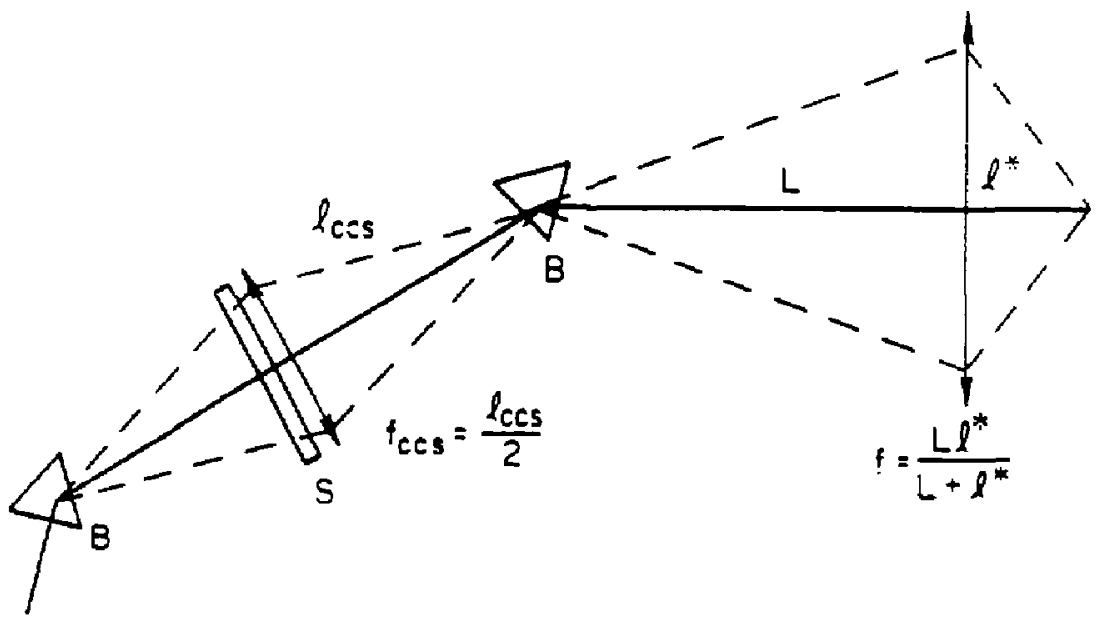

Figure 8. A simplified final focus section.

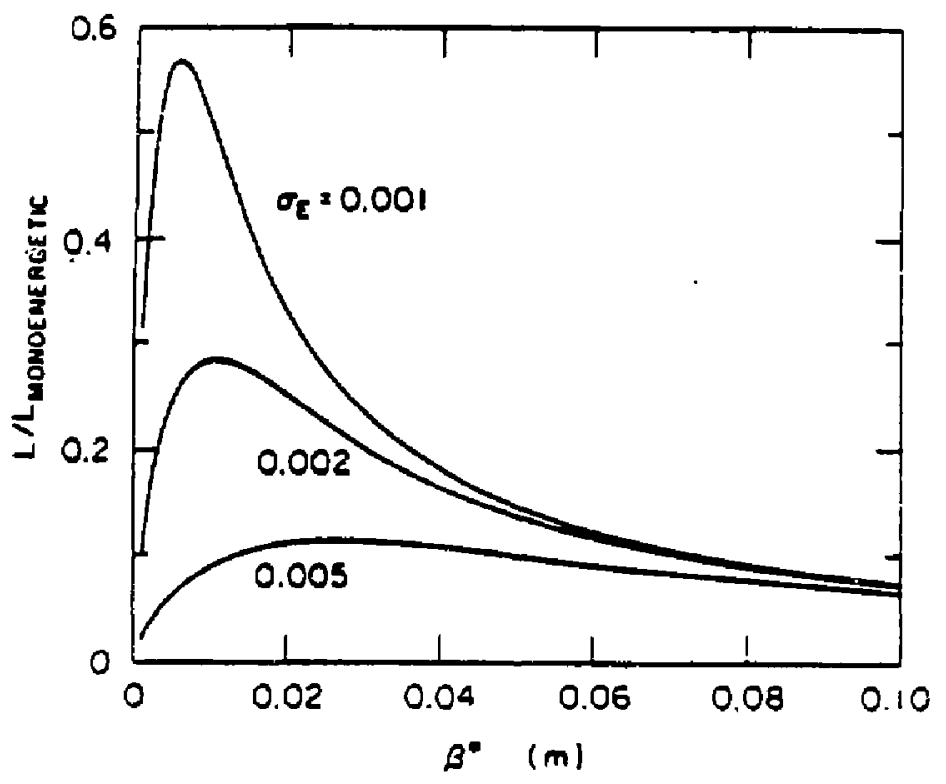

Figure. 9 Relative variation of the luminosity with the $\beta^{*}$-parameter in the final focus section, without chromaticity correction, and for several values of the energy spread. 

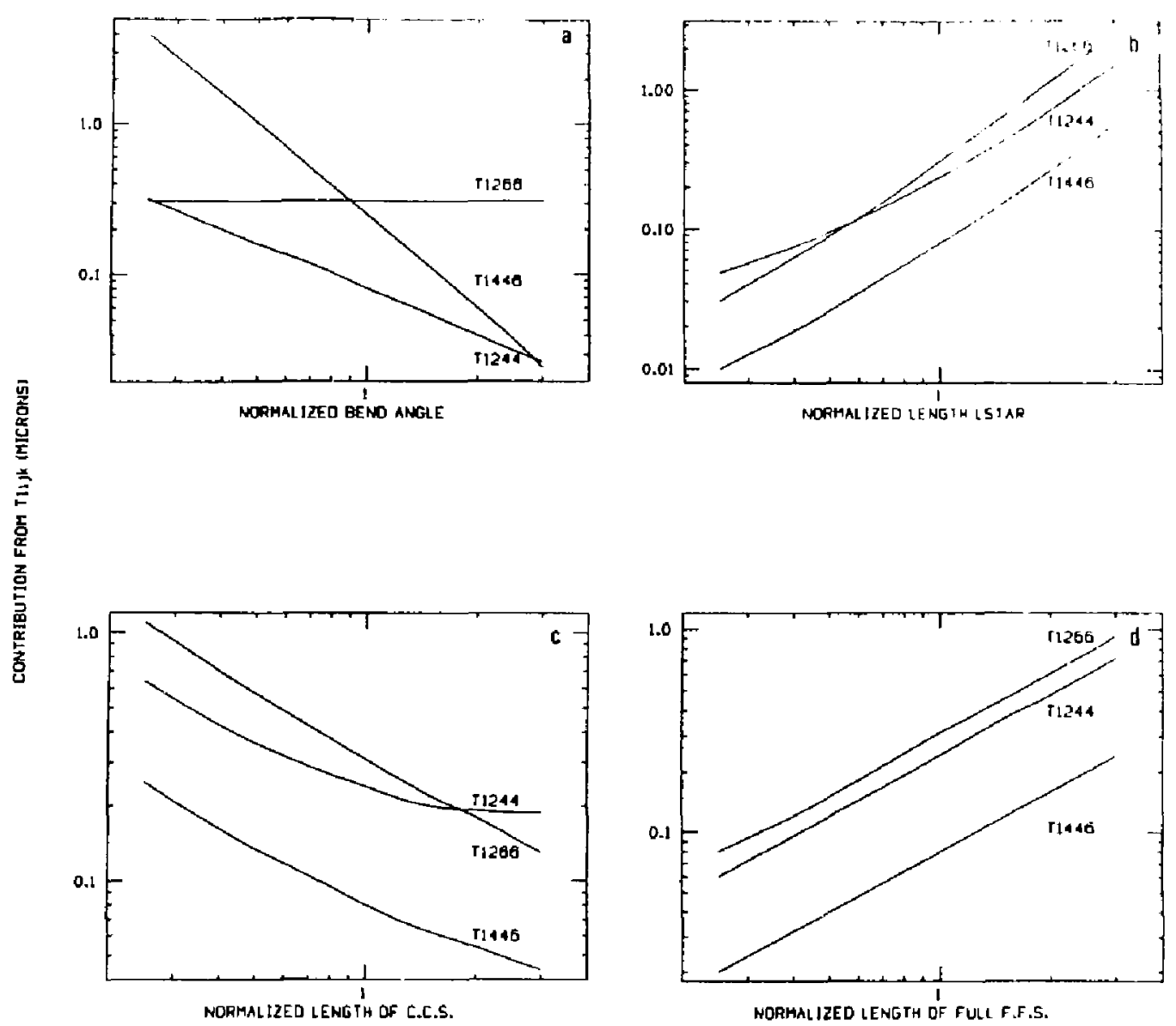

Figure. 10 Contribution to the interaction point beam size of the main second order aberrations, as a function of the bend angle of the chromatic correction section (a), of the distance bet ween the last quadrupole and the collision point (b), of the length of the chromatic correction section (c), and of tbe iotal length of the system (d). The real power dependances for each of these aberrations are:

(a) $T_{1286} \propto B^{0}, T_{1416} \propto B^{-1}, T_{1241} \propto B^{-2.05}$

(b) $T_{1 i j k} \propto\left(l^{*}\right)^{1.7 g}$

(c) $T_{1266} \propto\left(l_{\text {cce }}\right)^{-0.86}$

(d) $T_{1 i j k} \propto\left(I_{t o i}\right)^{-0.38}$

In the case of $(c)$, the power dependance is slightly weaker for $T_{1244}$ and for $T_{1446}$, for large values of $l_{\text {ccs. }}$. 


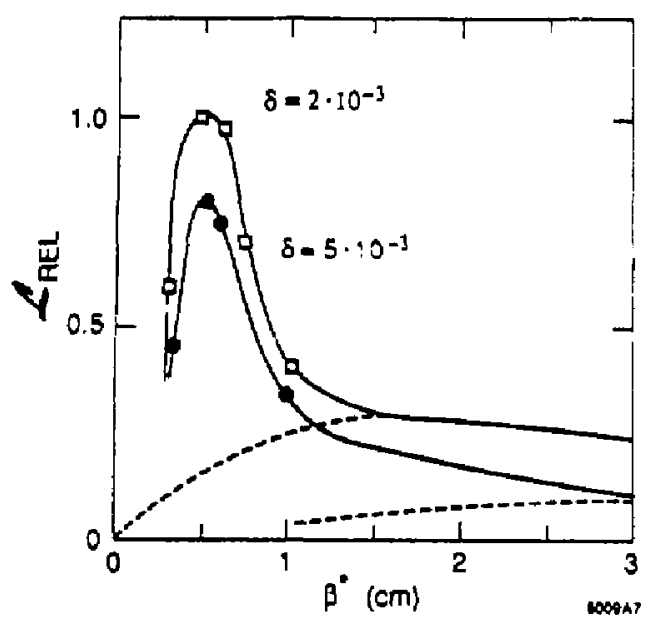

Figure. 11 Relative variation of the luminosity with the $\beta^{*}$-parameter, with chromatic correction (full line), and without chromatic correction (dotted line).
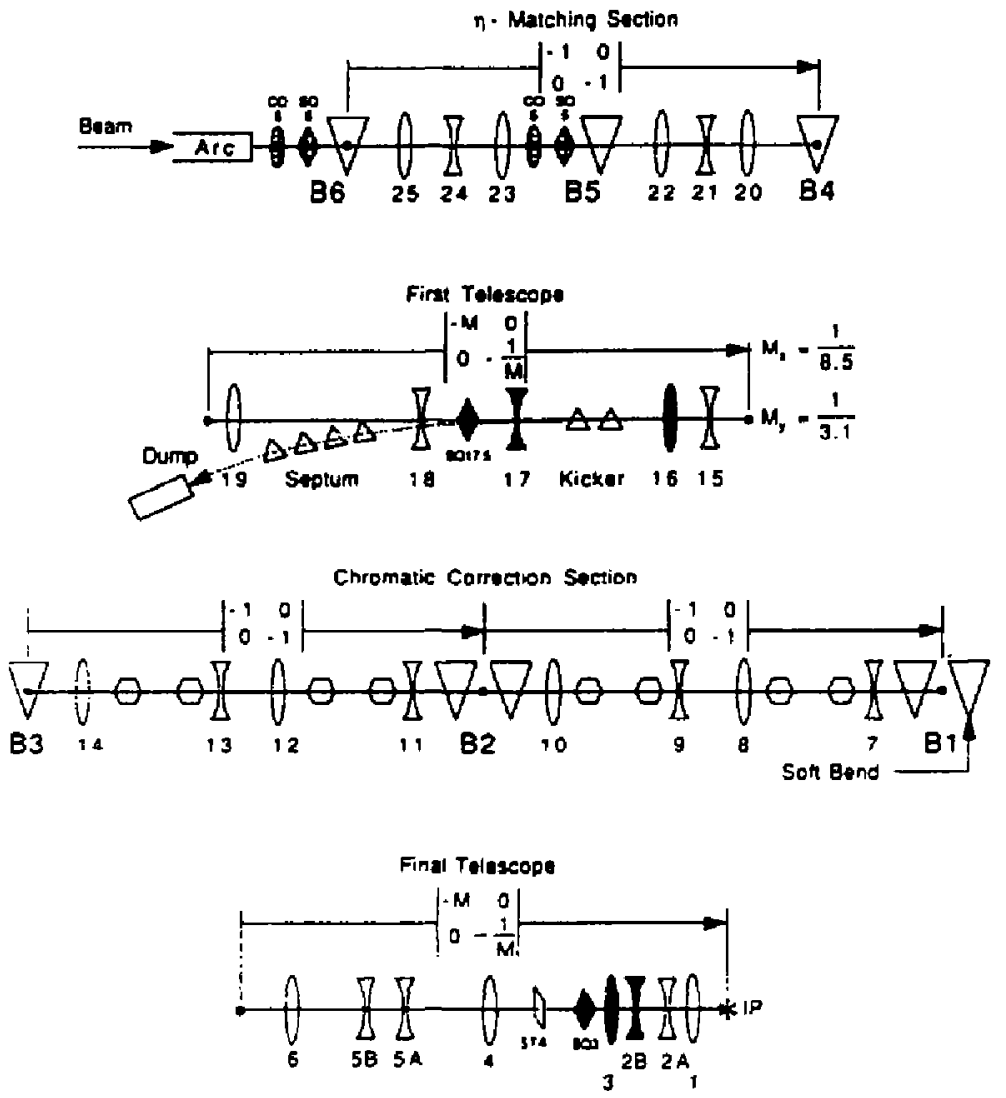

Figure. 12 Schematic of the final focus optics. Correctors for betatron matching are shown shaded, and for dispersion matching cross-hatched. 

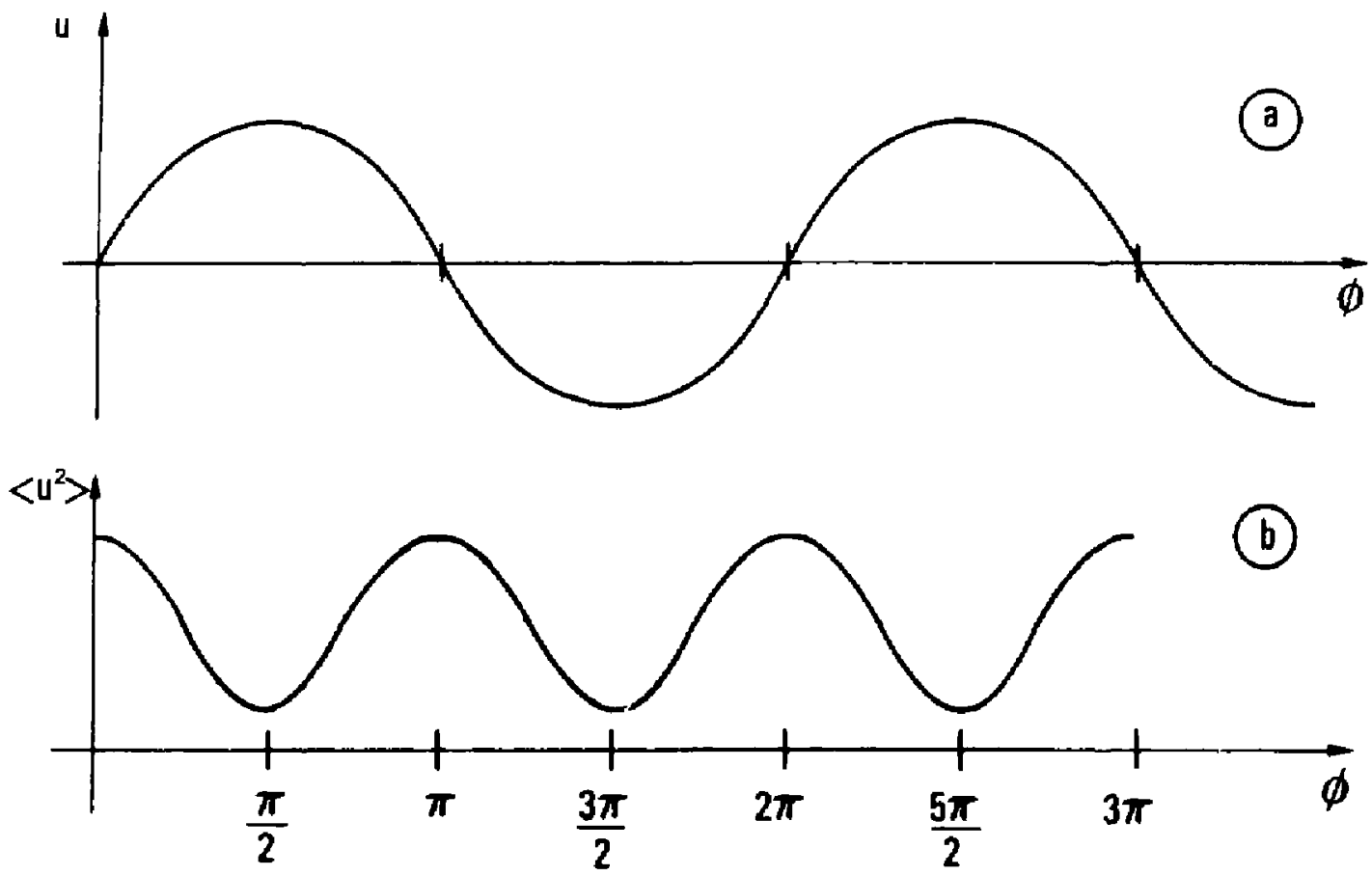

Figure. 13 Betatron oscillation (a) and ascillation of the square of the beam envelope (b) in the focusing lattice. The oscillation of the square of the envelope is at twice the betatron frequency.

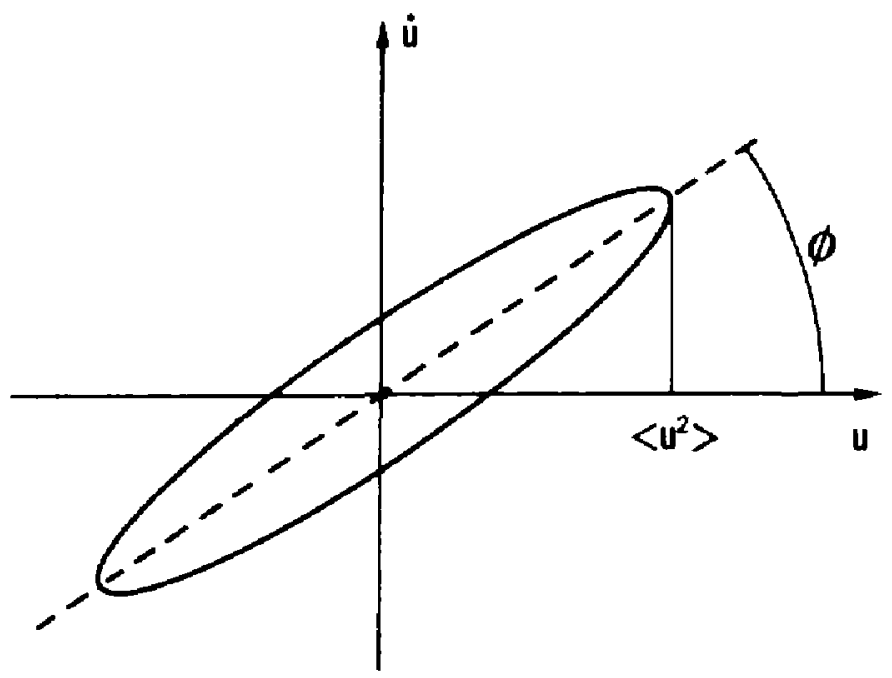

Figure. 14 Distortion and mtation of beam phase-space from a focusing error. 

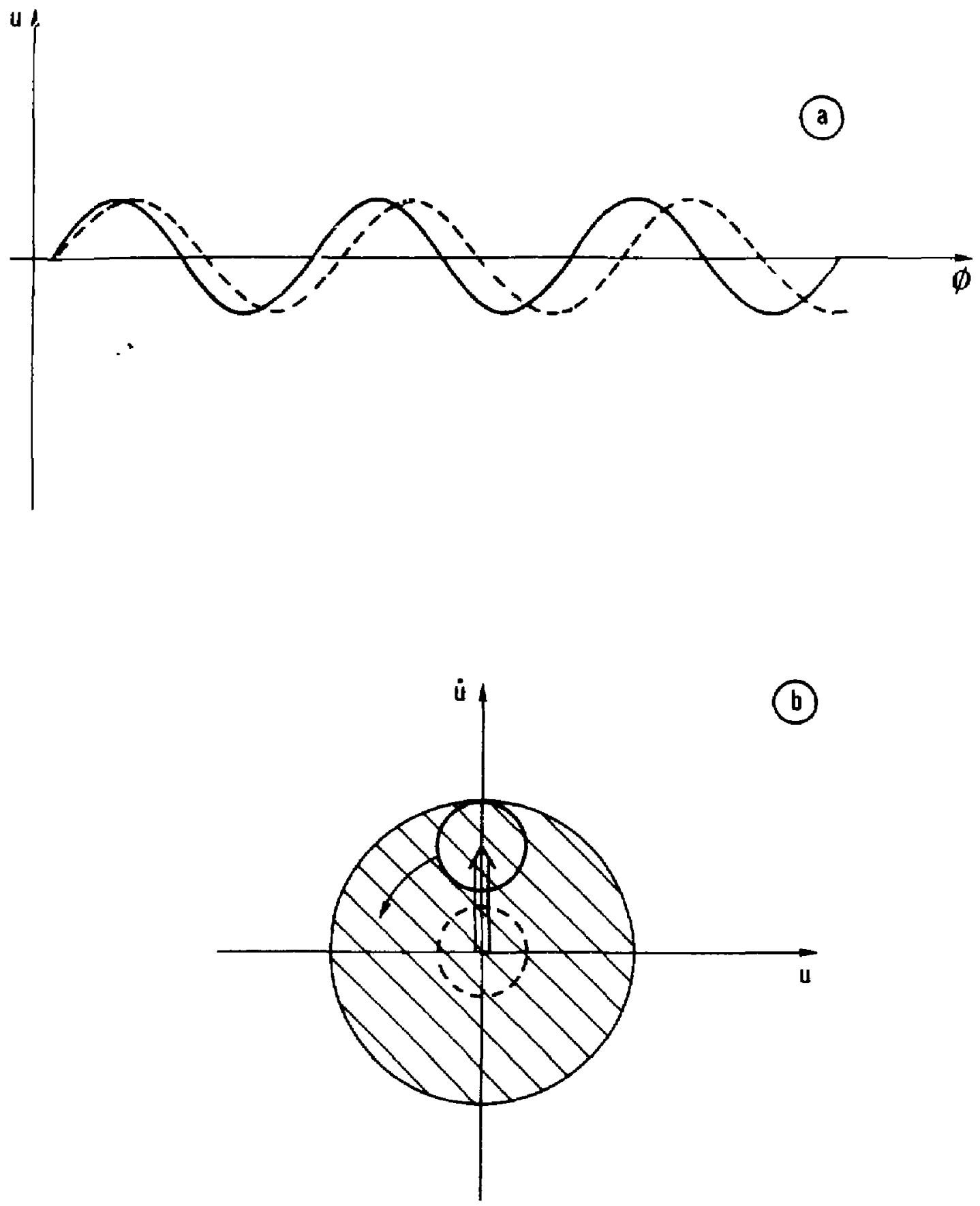

Figure. 15 Incoherent imaging of a betatron oscillation of a beam with finite energy spread (a). This resuits in an abnormal correlation between the transverse coordinates and the energy of the particles in the beam. The projection of the phase-space $\left(x, x^{\prime}, \frac{6 E}{E}, z\right)$ on the plane $\left(x, x^{\prime}\right)$ is thereby enlarged. 


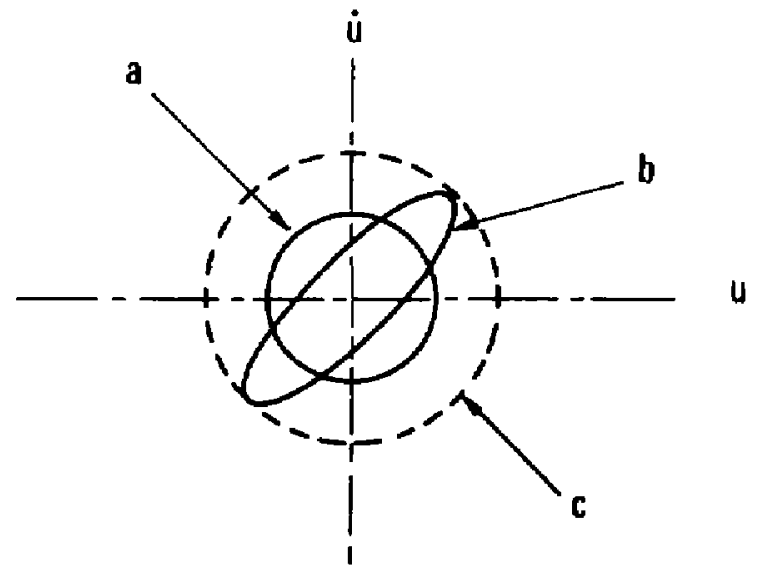

Figure. 16 Incoherent imaging of a phase-space distortion. In (a), we show the nominal phase-space, and in (b) the distortion of this phase-space from a focusing error. The incoherent imaging of this distortion generates a second order correlation between the transverse coordinates and the energy of the particles. When this correlation becomes large, the projection of the phase-space on the plane $\left(x, x^{\prime}\right)$ will correspond to the larger cercle in which the ellipse is inscribed.

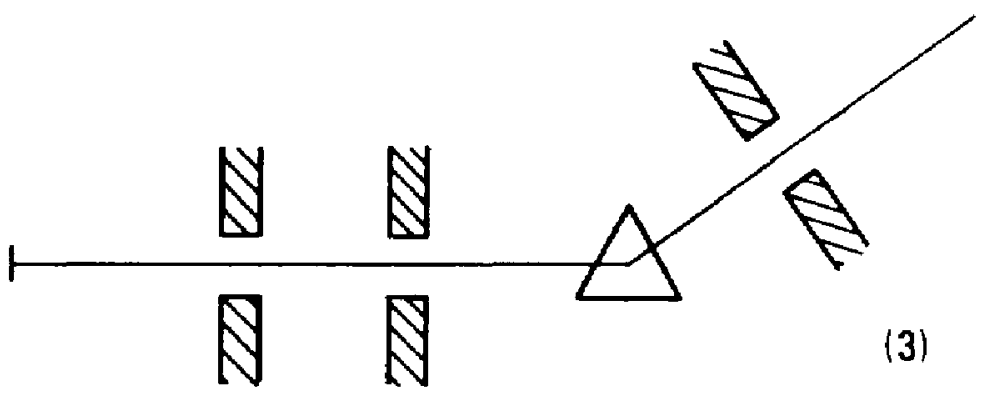

(0)

12)

Figure. 17 Basic principle of collimation. 


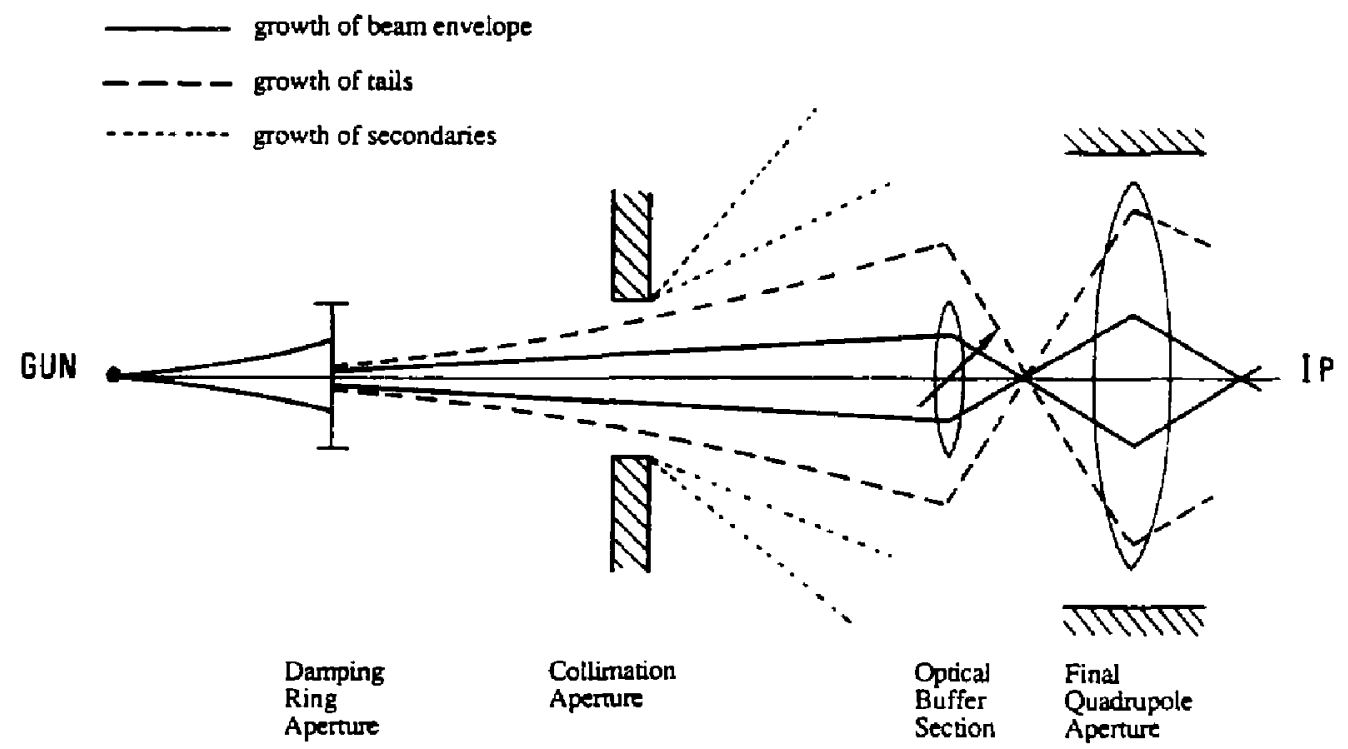

Figure. 18 Wustration of the separation in three major pieces of the tuning in the SLC. There are two breakpoints in the accumulation of the errors, from the radiation damping in the ring, and, in a more imperfect way, from the collimators at the end of the linear accelerator. 


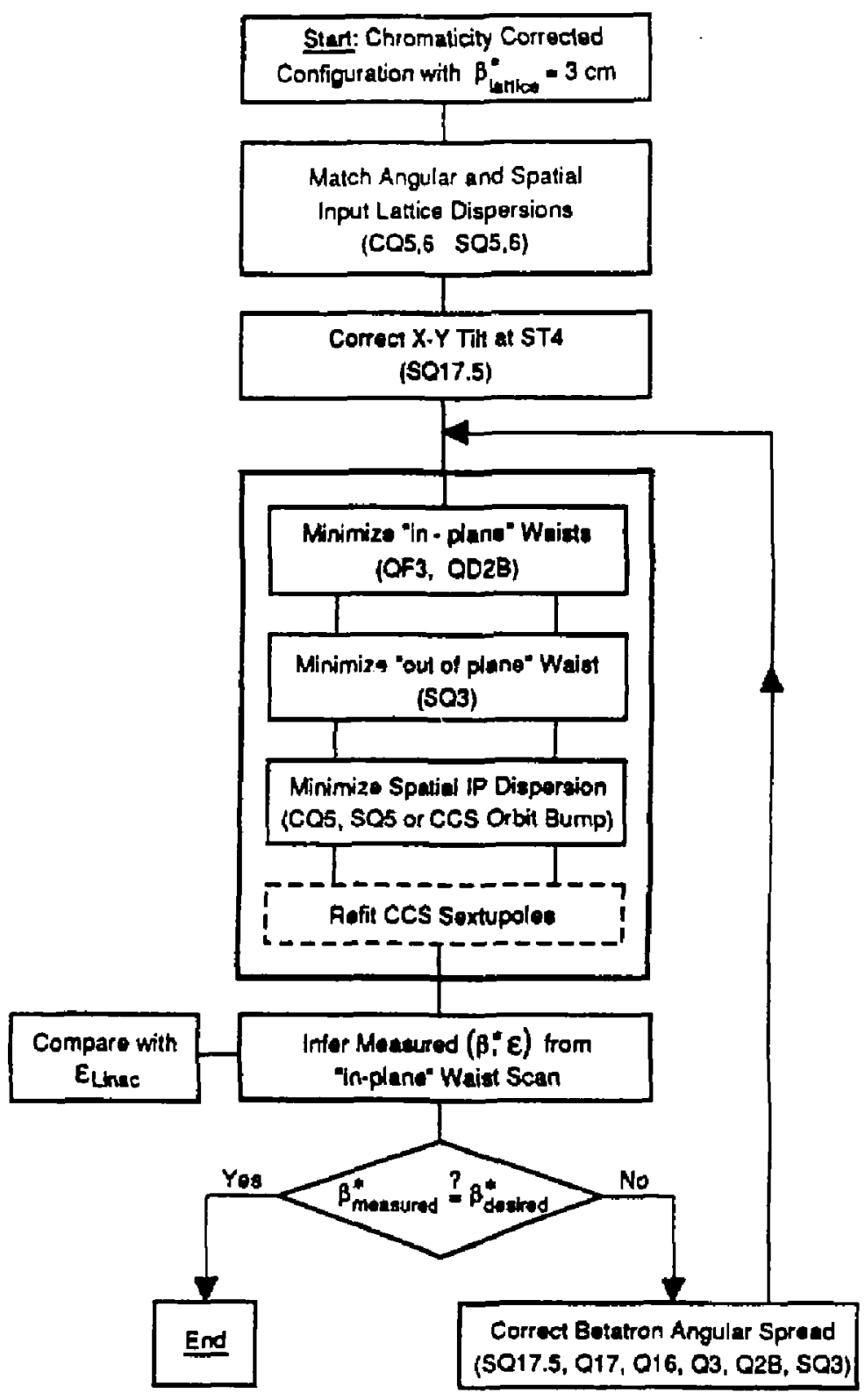

Figure. 19 Sequential application of the ten optical adjustments necessary to minimize the interaction point beam size. 


\begin{tabular}{l|c|c|c} 
& Design & Achieved & Unit \\
\hline$f$ & $120 \mathrm{~Hz}$ & $30 \mathrm{~Hz}$ & $\mathrm{~Hz}$ \\
\hline$\varepsilon_{\mathrm{N}}$ & $3.10^{-5}$ & $5.410^{-5}$ & m.rad. \\
\hline $\mathrm{N}\left(\mathrm{e}^{+}\right)$ & $7.10^{10}$ & $5.10^{9}$ & \\
$\mathrm{~N}\left(\mathrm{e}^{-}\right)$ & $7.10^{10}$ & $10^{10}$ & \\
\hline$\beta^{*}$ & 0.75 & 3 & $\mathrm{~cm}$ \\
\hline$\sigma^{*}$ & 1.5 & 4 & $\mu \mathrm{m}$ \\
\hline $\mathscr{L}$ & $2.310^{30}$ & $0.810^{27}$ & $\mathrm{~cm}^{-2} \mathrm{sec}-1$ \\
\hline
\end{tabular}

Table 1 


\begin{tabular}{|c|c|c|c|}
\hline & $\begin{array}{l}\text { Location } \\
\text { in SLC }\end{array}$ & $\begin{array}{c}\text { Consequence on } \\
\text { Phase-space }\end{array}$ & $\begin{array}{l}\text { Downstream Correc- } \\
\text { tability (Excluding } \\
\text { Background Constraint) }\end{array}$ \\
\hline $\begin{array}{l}\text { Synchrotron } \\
\text { Radiation }\end{array}$ & Arcs & Incoherent Dilution & $\mathrm{NO}$ \\
\hline \multirow{2}{*}{ Betratron } & \multirow{2}{*}{ R.T.L. } & $\left\langle x_{i}, \frac{\delta E}{E}\right\rangle$ & YES \\
\hline & & $\left\langle\mathrm{X}_{\mathrm{i}}, \mathrm{Z}\right\rangle$ & NO \\
\hline Oscillations & \multirow{2}{*}{ Linac } & $\left\langle X_{i}, \frac{\delta E}{E}\right\rangle$ & YES \\
\hline \multirow{2}{*}{ Errors } & & & NO \\
\hline & Arcs & $\left\langle X_{i}, X j\right\rangle^{*}$ & YES \\
\hline \multirow{2}{*}{ Guide-Field } & \multirow{2}{*}{ Final Focus } & $<X i, X j>$ & YES \\
\hline & & $\left\langle X_{j}, \frac{\delta E}{E}>\right.$ & YES \\
\hline \multirow{2}{*}{ Phase-Space } & \multirow{2}{*}{ R.F.L. } & $\left.<X_{i}, X_{j}\right\rangle$ & YES \\
\hline & & $<X_{i} X_{j}, \frac{\delta E}{E}>$ & NO \\
\hline \multirow[t]{2}{*}{ from } & \multirow{2}{*}{ Linac } & $<X_{i}, x_{j}>$ & YES \\
\hline & & $\left\langle x_{i}, x_{j} \frac{\delta E}{E}\right\rangle$ & NO \\
\hline Errors & Arcs & $<\mathrm{X}_{\mathrm{i}^{*}} \mathrm{X}_{\mathrm{j}}>$ & YES \\
\hline \multirow[t]{2}{*}{ Focusing } & \multirow{2}{*}{ Final Focus } & $<\mathrm{X}_{\mathrm{i}}, \mathrm{X}_{\mathrm{j}}>$ & YES \\
\hline & & $<X_{i}, \frac{\delta E}{E}>$ & YES \\
\hline
\end{tabular}

* The arc transfer is sensitive to steering for large steering errors. 
VI. REPORTS AND PUBLICATIONS ON OPTICAL CORRECTIONS IN THE ARCS 
This collider note describes one of the experimental methods used to determine the beam phase-space injected into the arcs. The method consists of:

1. Varying the phase-advance per cell in the optical lattice of the linear accelerator, in order to cause the beats in the $\beta$-parameter which result from the accumulation of focusing errors upstream of the arcs to move longitudinally, and of,

2. Measuring the beam-size on a fixed phosphor profile monitor screen.

This method is being successfully applied to monitor the phase-space at the end of the linear accelerator. 


\section{SINGLE PASS COLLIDER MEMO CN-367}

ALTHOR: Philip Bambade DATE: October 2, 1988

TITLE: BETATRON PHASE-SPACE DIAGNOSTICS

IN A FODO ARRAY*

\section{INTRODUCTION}

As has been shown ${ }^{2}$, betatron mismatch is conveniently diagnosed in long repetitive FODO arrays by systematically varying the cell phase-shift $\mu$ and by measuring beam size at the end. For small variations, lattice parameters $\alpha$ and $\beta$ change negligibly. This can be seen calculating ${ }^{2}$ for example $\beta$ as a function of $\mu$ in a thin lens FODO array. One gets:

$$
\beta_{ \pm}=\mathbb{L} \frac{1 \pm \sin (\mu / 2)}{\sin \mu}
$$

where $\beta_{ \pm}$are the minimum and maximum values, occurring at the $D$ and $F$ lens respectively, and $L$ is the cell length. The beam-size remains therefore nearly constant if the phase-space is matched to the lattice. If it is not matched, beam parameters beat at twice the betation frequency along the array, and the size at the end will vary as the beats are moved back and forth. $A$ total phase-shift of $\pi$ is needed to go through a full cycle of variation. In the Linac, where the total phase-advance is $60 \pi, a \pm 2.5 \%$ systematic variation of $F$ and $D$ lenses is sufficient.

In this note, we show by parametrizing the mismatch in normalized coordinates, that the beam phase-space is readily determined from the measured size-variations. The parametrization given is general and applies to any transfer channel. The insensitivity of lattice parameters to systematic phase-shift applies however only in a FODO array. In addition, the calculation assumes uncoupled motion. The proposed method is therefore not applicable in the Arcs or Final Focus Sections. It is relevant however at the end of the Linac, where it enables rapid diagnostic, in both planes simultaneously, and without extensive set-up, of the beam injected into the passive part of the Collider.

-Work supported by Department of Energy Contract DE-AC03-76SF00515. 
Phase-space parameters can also be determined without var ying any focussing element, through measurements at at least three different betatron phases. The four Sector-30 off-axis screens ${ }^{3}$ may be used for this, allowing monitoring without intercepting the beam during running. Practical formulas can be derived. An approximate expression is givtn for the emittance as an example.

As noted, the spacing in betatron phase of the four screens is not regular. Also, two out of fout screens are at $\beta_{\max }$ whereas the two others are at $\beta_{\min }$. This gives about a factor two assymetry in their sensitivities, which the error analysis of this method must take into account. This, and a comparison with the traditional methods ${ }^{5}$ is rot included here.

\section{NORMIALIZED COORDINATES 5,6}

The transverse motion of a particle in a focussing array is governed by Hill's equation:

$$
\frac{d^{2} z}{T^{2}}+k_{2}(s) z=0
$$

where $z$ and $s=c t$ are the tra.sverse and longitudinal coordinates respectively, and $k_{z}(s)$ represents the strength of the time-varying restoring force from the focussing array.

Introducing the normalized variables $\tilde{z}=\frac{x}{\sqrt{\beta}}$ and $d \phi=\frac{d}{\beta}$, where $\beta$ satisfies:

$$
\frac{1}{2} \beta \frac{d^{2} \beta}{d s^{2}}-\frac{1}{4}\left(\frac{d \beta}{d s}\right)^{2}+k_{s} \beta^{2}-1=0
$$

transforms the governing equation (2) ints that of a pure harmonic oscillator:

$$
\frac{d^{2} \bar{z}}{d \phi^{2}}+\bar{z}=0
$$

Thus the solution of $(2)$ is:

$$
z=a \sqrt{\beta} \cos (\phi+b)
$$

where $a$ and $b$ are integration constants. 
It is easily verified that:

$$
\gamma z^{2}+2 \alpha z z^{\prime}+\beta z^{\prime 2}=a^{2}
$$

where $z^{\prime}=\frac{d x}{d x}, \gamma=\frac{1+\frac{\omega^{\prime}}{\alpha}}{\beta}$, and $\alpha=\frac{-\beta^{\prime}}{2}$. The quantity in (6a) is called the Courant-Snyder invariant. It can also be written in matrix form:

$$
\left(z, z^{\prime}\right) \mathrm{T}^{-1}\left(\begin{array}{l}
z \\
z^{\prime}
\end{array}\right)=a^{2}, \text { where } \mathrm{T}=\left(\begin{array}{cc}
\beta & -\alpha \\
-\alpha & \gamma
\end{array}\right) \text { and } \operatorname{det}(\mathrm{T})=1
$$

(6) defines the closed phase-space trajectory - an ellipse - of a particle with initial condition a. It also defines the envelope of the phase-space matched to the lattice. The Twiss parameters $\alpha, \beta$ and $\gamma$ are here properties of the lattice. The area $\pi a^{2}$ of the ellipse is identified as $\pi$ times the emittance $\epsilon$. In the matched condition, the beam-matrix ${ }^{7} \sigma=\epsilon \mathrm{T}$.

\section{PARAMETRIZATION OF MISMATCH}

In the normalized coordinates $\bar{z}=\frac{z}{\sqrt{\beta}}$ and $\bar{z}^{\prime}=\frac{d \bar{\alpha}}{d \phi}=\sqrt{\beta}\left(z^{\prime}-\frac{\beta^{\prime}}{3 \beta} z\right)$, the matched phase-space is a circle of radius $\epsilon$. This can be verified by direct substitution in (6a). A mismatch amounts to distorting this circle into an ellipse. We characterize the mismatch by the ratio $M$ of the radius of the larger circle in which the ellipse is inscribed to that of the initial circle corresponding to the matched case, and by the angle $\phi_{0}$ between its major axis and the abscissa (see Fig. 1): $M$ and $\phi_{0}$ can be thought of as the splitude and phase of the mismatch.

The equation of the distorted ellipse is calculated in terms of $M$ and $\phi_{0}$, first in the coordinates rotated by $\phi_{0}$ in which it is erect, and then transforming back in to the unrotated coordinates. This gives:

$\tilde{z}^{2}\left(M^{2} \cos ^{2} \phi_{0}+\frac{1}{M^{2}} \sin ^{2} \phi_{0}\right)+\left(\tilde{z}^{\prime}\right)^{2}\left(M^{2} \sin ^{2} \phi_{0}+\frac{1}{M^{2}} \cos ^{2} \phi_{0}\right)+2 \tilde{z} \tilde{z}^{\prime} \cos \phi_{0} \sin \phi_{0}\left(M^{2}-\frac{1}{M^{2}}\right)=\epsilon$.

The corresponding beam-matrix, written in the normalized system, is:

$$
\bar{\sigma}=\epsilon\left(\begin{array}{cc}
M^{2} \sin ^{2} \phi_{0}+\frac{\cos ^{2} \phi_{0}}{M^{2}} & -\cos \phi_{0} \sin \phi_{0}\left(M^{2}-\frac{1}{M^{2}}\right) \\
-\cos \phi_{0} \sin \phi_{0}\left(M^{2}-\frac{1}{M^{1}}\right) & M^{2} \cos ^{2} \phi_{0}+\frac{\sin ^{2} \phi_{0}}{M^{2}}
\end{array}\right)
$$


Transforming back into real coordinates gives:

$$
\left\{\begin{array}{l}
\sigma_{11}=\beta \bar{\sigma}_{11}, \\
\sigma_{12}=\tilde{\sigma}_{12}-\alpha \bar{\sigma}_{11}, \\
\sigma_{23}=\frac{1}{\beta}\left(\bar{\sigma}_{23}+\alpha^{2} \bar{\sigma}_{11}-2 \alpha \tilde{\sigma}_{12}\right) .
\end{array}\right.
$$

In the matched condition, characterized by $M=1$, (9) reduces to (6b) as expected.

\section{PHASE-SPACE DETERMINATION}

From (8) and (9), the beam-size measured at an arbitrary point at the end of the array an a function of the total induced phase-shift $\phi$ is:

$$
\sigma^{2}(\phi) \simeq \frac{\epsilon \beta}{2}\left(\left(M^{2}+\frac{1}{M^{2}}\right)+\left(M^{2}-\frac{1}{M^{2}}\right) \cos 2\left(\phi-\phi_{0}\right)\right) .
$$

The period of this function is $\pi$ as expected. The mismatch parameters $M, \phi_{0}$ and the emittance $\epsilon$ are easily computed:

$$
\left\{\begin{array}{l}
\epsilon \simeq \frac{\sigma_{\text {Ming }}}{\beta}, \\
M^{2} \simeq \frac{\sigma_{\text {Min }},}{\sigma_{\text {Mat }}}, \\
\phi_{0} .
\end{array}\right.
$$

In (11), $\phi_{0}$ is the difference in phase between $\sigma(\phi)=\sigma_{\text {Max }}$ and the starting point $\sigma(0)$.

The full beam-matrix can then be zeconstructed from (9), using the lattice parameters $\alpha$ and $\beta$ at the measurement point. Equations (10) and (11) are approximate because Twiss parameters $\alpha$ and $\beta$ change slightly with the induced phase-shift from (1). The contribution to the error from this is however small as it is bounded by their variation over the $2.5 \%$ phase changes which are applied.

We can also use (10) to determine the phase-space from measurements on the four off-axis screens ${ }^{3}$, without varying any focusing element. Approximate expressions are easily written if the slight mismatch present in the design lattice is neglected $^{\dagger}$. Using $\Delta \phi_{12} \simeq \Delta \phi_{34} \simeq 22.5^{\circ}$, and $\Delta \phi_{23} \simeq 67.5^{\circ}$, where $i=1,4$

t The deaign Liase lattice is not fully periodic. It ineludes deviations in quadrupole strongtha required to match acroes rector boundaries. Tbese local deviations can be as large as 20 $\%$. However because the syntematic strength variations induced in this method are about $2.5 \%$, effectu trom these irregolarities are omall. This has been checked ${ }^{l}$ using the on-line model. 
refer to the four screens, the horizontal electron emittance is:

$$
\epsilon^{2}=\left(\frac{A_{1}+A_{2}+A_{3}+A_{4}}{4}\right)^{2}-\left(\frac{A_{1}-A_{3}}{2}\right)^{2}-\left(\frac{\sqrt{2}\left(A_{2}-A_{4}\right)-\left(A_{1}-A_{3}\right)}{2}\right)^{2},
$$

where $A_{i}=\frac{\sigma_{3}^{2}(i)}{\beta_{i}}$, in which $\beta_{1,3}=\beta_{\min } \simeq 20 \mathrm{~m}$. and $\beta_{2,4}=\beta_{\max } \simeq 50 \mathrm{~m}$. The same expression applies for the vertical plane by exchanging $\beta_{\min }$ for $\beta_{\max }$, and for the positron emittance, by exchanging horizontal for vertical electron results.

Similar expressions can be obtained in the same way for mismatch parameters $M$ and $\phi_{0}$.

\section{ACKNOWLEDGEMENTS}

I wish to thank K. Brown, J. Haissinski, T. Himel, T. Lohse, J. Seeman and J. Sheppard for useful comments on this method.

\section{REFERENCES}

(1) T. Himel, Private Communication (August 1988).

(2) For Example: E. Wilson in: "Circular Accelerators - Transverse", SLAC Summer School on Physics of Particle Accelerators (July 1985), AIP Conference Proceedings 153, page 21.

(3) J. Seeman et al.; "Transverse Wakefield Control and Feedback in the SLC Linac", IEEE Particle Accelerator Conference, Washington, D.C.(March 1987).

(4) J. Seeman, Private Communication (August 1988)

(5) J. Rees and L. Rivkin, "On Measuring Emittances and Sigma Matrices", SLAC-PUB-3305, (March 1984), and M. Ross et ad., "High Resolution Beam Profile Monitors in the SLC", SLAC-PUB-3640 (April 1985).

(6) E. Courant and H. Snyder, "Theory of the Alternating Gradient Synchrotion", Annals of Physics, Vol. 3,1-48 (1958).

(7) K. Brown et al., "TRANSPORT", SLAC-91, Rev. 2 (May 1977). 


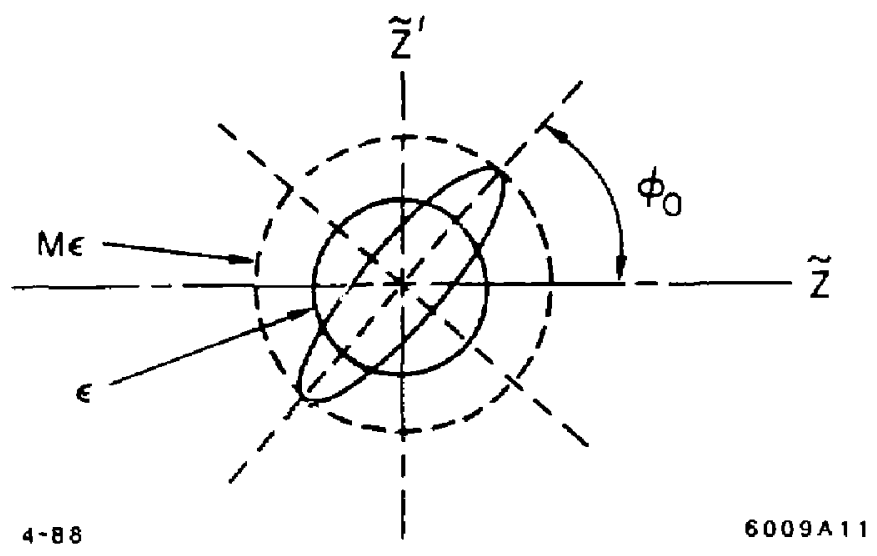

Fig. 1: Matched (inner circle) and mismatched (ellipse) phase-space in normalized coordinates. 
VI.2 "Roll-flx - An Adiabatic Roll Transition for the SLC Ares"

This conference article describes the modification of the roll transitions which were used to provide the vertical deflections necessary to follow the terrain of the SLC site. The boundary was modified into a smoother transition, which almost perfectly suppressed the coupling of horizontal lattice dispersion into the vertical plane, and substantially reduced the sensitivity of the betatron lattice to systematic focusing errors. This modification was proposed and implemented to help control the magnification and build-up of errors from the arc lattice, by making the system more error-tolerant. The paper summarizes the evaluation and performance of this scheme. 


\title{
ROLLFDX - AN ADIABATIC ROLL TRANSITION FOR THE SLC ARCS:
}

\author{
P. Bambade, K. Bhow, T. FiEgUTh, A. Hutton, D. RITSON, M. Sangs and N. TOGE \\ Slanford Linear Acceleralor Center, Slanjond Universty, Slanford, Coliforma 94909
}

Contuidated to the IEEE Particle Acceleralor Conjerence, Caicugo, Illinois, Morrh 20-29, 1989.

\begin{abstract}
The SLC Ares were rolled at achromat boundaries to follow the terrain of the SLAC gite. This makes the linear optics sensitive to systemat:c geadient ertors, from which severe crossplane coupling effects may arise. As a partial correction, a smoother roll transition was introduced which relieves much of this sensitivity. We present an evaluation of this scheme and seport on the observed improvements.
\end{abstract}

\section{INTRODUCTION}

The two Ares of the Stanford Linear Collider (SLC) are designed to bend the elexirons and positrons around and into collision, without significant emittance dilution.' To minimize emittance growtb from syncbrotron radiation in the bend field, the focusing must be strong ard compact and, therefore, use combined function magnets. To asure achromatic imaging. the magnets must also include sextupole componests, and be grouped into sets (achromsts) appropriately symmetrized for the suppression of optical aberrations. ${ }^{2}$ In addition, for economical reasons, the two Arcs were designed to follow the terrain ol the SLAC site (see Fig. 1), and thus include vertical deflections. For maximum compactness, these deflections were produced by rolling the magoets around their axis by up $1010^{\circ}$ at achromal boundasies (see Fig. 2). To provide an overall cancellation of the induced cross-plane coupling, the rolls were grouped in pairs separated by one or several achromats, corresponding in the ideal system to an identity transfer matrix with $6 n \pi$ phaseadvance.
However, these long-cange capcellations resuited in a limited bandwidth for the optical transfer, which had relatively st tingent tolerances to systematic focusing ettors. This lact was realized in several stages, before and during the initial beam tests. At first, stringent tolerance to syatematic horizontal displacement errors, which in the combined function magnets generate systematic focusing errors. was doticed through computer simulations. For example, see Ret. 3.

During the first beam tests, it was observed that the transfer of betatron oscillations and of the dispersion function across rolled achromat boundaries could be asuociated with a large magnification. A detailed calculation of the magnification associaled with this transfer can be found in Ref. 4 . In the initial commissioning, this magnification was observed to be as large as a factior of three over the whole length of the Arc because the systernatic errors exceeded the specified design tolerances of 0.602 . $^{3}$

Althol:gh, for the case of equal input emittances (corresponding to the SLC design specification), such gtowth is in principle recoverable downstream, in the Final Focus, where a set of skew corrections are jostalled, ${ }^{6}$ its megnitude required inilial correction within the Arcs. Aloo, as was later found, the projected trangverse $r$. ittance must in fact be close to the aominal desiga value at the exit to the Areg, in order to minimize detector backgrounds induced by beam-tails striking the smafler (notmalized) aperture in the Final Facus region. Two basic cures were therefore devised. The first consisted of adjusting the
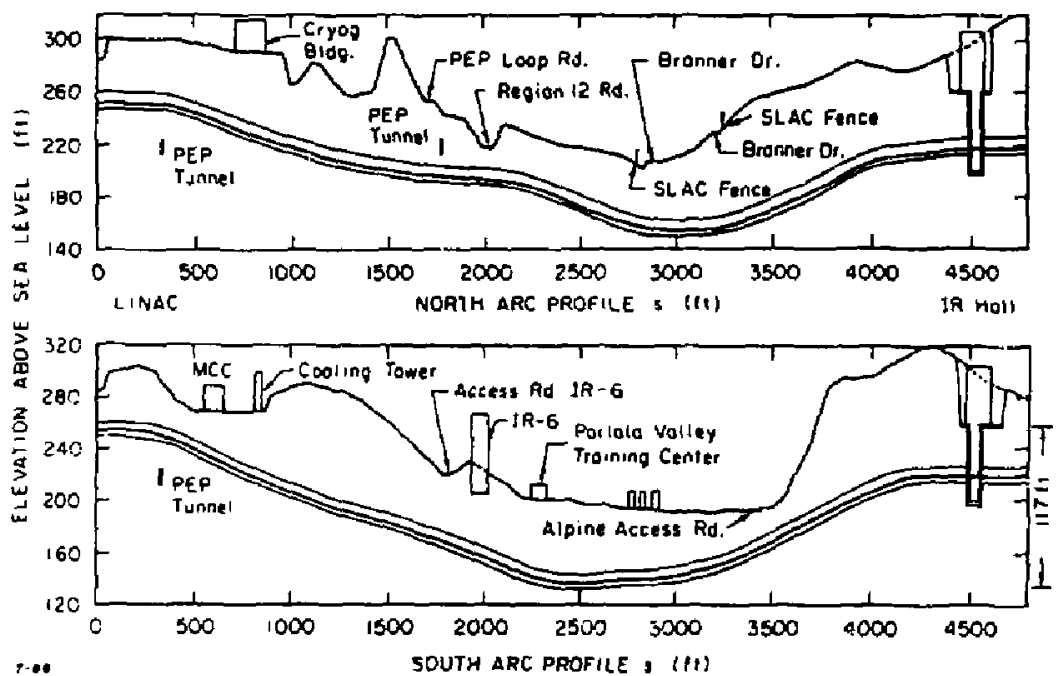

Fig. 1. Vertical profiles of the SLC Arss.

* Work supported by the Deputment of Energy, cont ract DE-AC03-7ASF00515

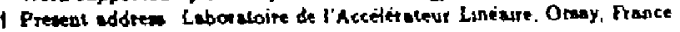




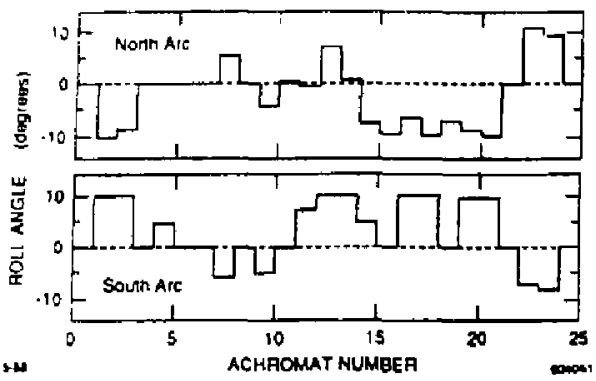

Fig. 2. Roll angle aboul beam aris veraw achromat number for North and South Ares.

Focusing gradienta, based on mearurements of the phye-edvance in each achromat, to their nominal values. Sueb edjustmente belped bring the growth in the betatron transfer down to within - fuctor of about two, but further seductions by this procedure were limited by menuremeat errors and by the leck of fully separate controls in each plene and in each echromat."

A verond cure convisted of splitting each roll over weveral maguete on each side of the boundries, to yield inoother transitions having - grester tolerance to phasedvance errors, and to nearly supprea the coupling of horisontal latice dispersion into the vertical plane. The adjustments of the phese edvance bad brought the syoten close to specification, and had miniraized the coupling to the point where it could be handled relatively well in the Final Focus. It wa however felt important for future operability la implement this pusive nollfis cure. which makes the oyatem sigaificantly more eror-tolerant, particularly for equal or close to equal jnitial emituances in both planes, as was noted above.

In addition, the neariy uppreased vertical dispernion is expected to reduce synchrotron radiation induced emittance growtb in the vertical plane. The scheme could be installed without major disruption to the benm-line, and roulted in some observed improvements. The reference trajectory could be kept unperturbed througb andl vertical displacements of the magnets involved.

In this paper, after introducing an epproximate measure for the cross-plane coupling in the betatron tranafer, we evaluate the seasitivity to errors in the initial and modifed desigas, and characteriz: the predicted inprovements. We theo repori on observed improverneots.

\section{APPROXIMATE CHARACTERUZATION OF CROSS-PLANE COUPIING}

The practical consequences of crom-plane coupling are different in a beam-line than in a circular machine. In a circular mechine, the motion stable only for tune wuch tbat num-rewonances (corresponding to $\mathrm{pw}_{\mathrm{x}}+\mathrm{q} \mathrm{w}_{\mathrm{y}}=\mathrm{n}$ ), have negligible effects. Residual coupling arise in this case exelusively from difference-resonances (correaponding to $p \nu_{z}-q \nu_{y}=n$ ), which can be shown to result in alable beating between the two projected transverse emittuaces. In a beam-line, distortions from cross-plane coupling can correspond both to growing and decaying solutions. ${ }^{10}$, It The two projected emitunces can in this case botb grow. It hes been shown that the severity of such grawth cas be characterized by the determinant of the off-diagond two-by-two submatrix $\mathrm{C}$ of the general four-by-four ireosfer matrix: 10

$$
R=\left(\begin{array}{llll}
R_{11} & R_{12} & R_{13} & R_{11} \\
R_{21} & R_{22} & R_{23} & R_{21} \\
R_{31} & R_{32} & R_{33} & R_{34} \\
R_{11} & R_{43} & R_{43} & R_{41}
\end{array}\right)=\left(\begin{array}{ll}
A & B \\
C & D
\end{array}\right)
$$

This cap be seen from calculating the projected emultances $\epsilon_{x}$ and $c_{y}$ onto eacb plane of a four dimensional phase-space transfered througb a fully coupled system, and by using the six symplectic conditions imposed by Hamiltajian Mechanics." With the simplifying saumption of upright phase-elipses and of beams initially uncoupled in both planes, one cas show that the following inequality holds: ${ }^{10}$

$$
e_{x, y} \geq c_{x, y}(0)|1-\operatorname{det} \mathrm{C}|+e_{y, x}(0)|\operatorname{det} \mathrm{C}|
$$

In most canes, the inequality sign in Eq. (2) can be repluced by 2 equality. This has been observed in several computer simulationa. An andytical attempt to show this is rescribed in the Appendix.

From $E_{q}$. (2), we that the most severe coupling effect: arice if: $\operatorname{det} \mathrm{C}<0$, or if: det $\mathrm{C}>1$. In this case, the phasespece projections will grow in botb planes, itrespective of the initia emittance vilues. Oo the other hand, coupling effects with $0<\operatorname{det} \mathrm{C}<1$ are evere only if the initial emittance valuea are very anymetric (and if it is desirable to prenerve auch n enymetry). In this cue, the coupling will kend to equalize the two emittince projection. In the SLC, where the initial emiteraces are close to equal, coupling effects with $0<$ det $C<1$ are beniga.

\section{DESCRIPTION OF ORIGINAL AND TAPERED ROLL TRANSITIONS ${ }^{15}$}

Figura $3(a)$ and (b) thow the principle of the original and tapered roll tranuition pain. The firat tranaition bas \& total angle $A$. It is matched by a second trancition, witb a total andle $-\theta$, located an integer number of echromats downatream. In this way, all cros-plane coupling effects cancel after th: second tranistion, if the achromnts in between are perfect, and corrempond to en ideptity transier matrix.

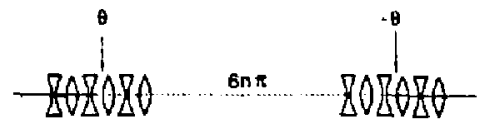

(a)

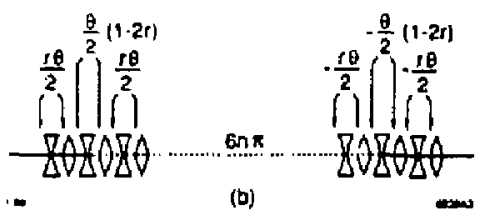

Fige. S(a) and (b). Principle of original (a) and tapered (b) rol trantion pairs. In the original set-up (a). pairs of rolis wei concentmited at achromat doundaries separated by 6 ax betatro; chace-adoance. In the tapered solution ( 6 ), each roll vas spli abol five mognets to yield a smoother transition. The optimisi. oulue for the ratio $\mathrm{I}$ of the total roll of the first call to the tota roll of the transition is near $\mathrm{r}=0.28$.

In the origind transition [see Fig. $3(a)$ ), the full roll i concentrated at the achromat boundary. In the tapere transition [ee Fig. 3(b)], the rolls are distributed across thrcells around the boundary. As we will show, this taperin mppreses the most damaging component of tbe cross-plar coupling induced in the betatron transier. Within each cell the rolls are split equally across the defocussing magnet. Thi was found to nearly cancel the coupling of horizontal lattic dispersion into the vertical plane, and is due to the fact tha the vercical phase-advance across a defocusing magnet is onl about $22^{\circ}$, and because the angular horizontal lattice dispersion 
has opposite sign at the entrance to focusing and to defocusiog magaets. We define by $r$ the ratio of the roll of the firat cell to the total roll of the iransition. In the original proponal, $r=0.38 \mathrm{was}$ used. by analogy with the coefficiente for a matched trajectory bump. It was later found that $r=0.28$ gives a slightly better Tesuits. ${ }^{24}$

\section{TOLERANCES WTTH ORIGINAL AND TA PERED ROLL TRANSITIONS}

Following the above deacription of crowe-plane coupling effects. we use the magoitude and the aign of det $\mathrm{C}$ to char acterize the severity of the crosu-pine coupling effects which arise from errors in the Arc lattice.

The original deaign mat especially wensitive to the total

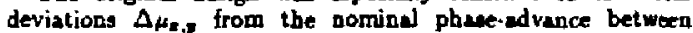
trangition pairs. For two original roll tranaitione, each of angle A, and exparsed by a regular FODO lattice wilh an integer number of betatron periods [us in Fig. 3(a)], it can be shown thas after the second tranoition

$\operatorname{det} C=\sin ^{3}(2 \theta)\left[\sin ^{2}\left(\frac{\Delta \mu-\Delta \mu_{y}}{2}\right)-\alpha^{2} \sin \Delta_{\mu_{x}} \sin \Delta \mu_{y}\right]$,

where $\alpha$ is the usual Twiss purasater at the transition (in the Are, $\alpha=2.65 \mathrm{~T}$.

For phase-advance errors with the rame sign in each plane, det $\mathrm{C}<0$, while for phase-advance errors with opposite sigs, det $\mathrm{C}>0$. The magaitudes of det $\mathrm{C}$, computed with simulation ${ }^{15}$ to confirm Eq. (5), are shown in Fig. 4 as a function of $(1 / 2)\left(\Delta \mu_{x} \pm \Delta \mu_{y}\right)$, for a typical tranition with $\theta=10^{\circ}$. Ao expected, the maximum value for $\mid$ det $\mathrm{C}$, which in elose to one, is reached for $(1 / 2)\left(\Delta \mu_{x} \pm \Delta \mu_{m}\right)= \pm 90^{\circ}$. The unme quantity is ahow $d$ in Fig. 5 for the tapered transition. As can be onset of a negative det $C$ is searly suppreased by the tapered transition, and the onset of a positive det $C$ is reduced by a factor of two

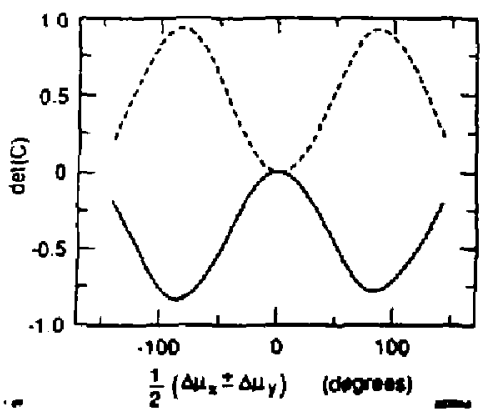

Fig. 1. det $C$ as a function of systematic phase-adoance errors of equal sign (dashed fine) and of opposile sign (solid line), bet ween two onginal roll transitions.

The actual roll dintributions in the tro Arc are mose complicated (we Fig. 2), but are euperpotitions of the bue ones abown in Fig. 3. We therefore expect the wine overall features as for the aimple examples exwmined above. To verify this, we show in Figs. 6 and 7 , the ame quantities a in Figs. and 5 , under the same conditions - but for the whote North Arc, as a function of the fractional phaseadvance devitutions $(1 / 2)\left[\left(\Delta \mu_{x} / \mu_{x}\right) \pm\left(\Delta \mu_{y} / \mu_{y}\right)\right\}$. As cap be seen is Fig. 6 (solid line), the tolerance to ayutematic phese-advance errors, for negligible coupling to occut, was about \pm 0.006 in the origind North Ars.

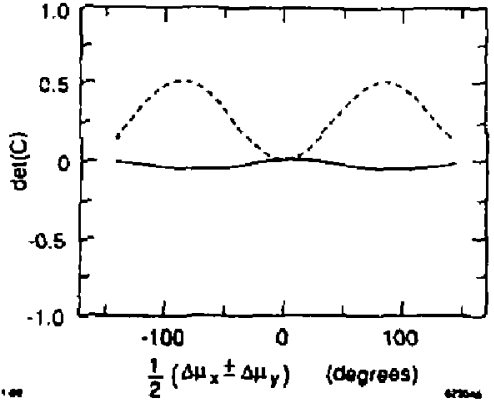

Fig. 5. det $C$ as a function of systematic phast-adtance errors of equal sign (deshed line) and of opposite sigh (solid line), between two tapered roll transitions.

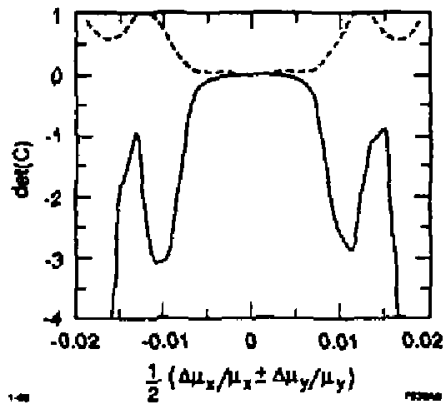

Fig. 6 . det $\mathrm{C}$ as a function of systemafic phase-advance errors of equal sign (dashed line) and of opposite sign (solid line) in the entire North Are with original roll transitions.

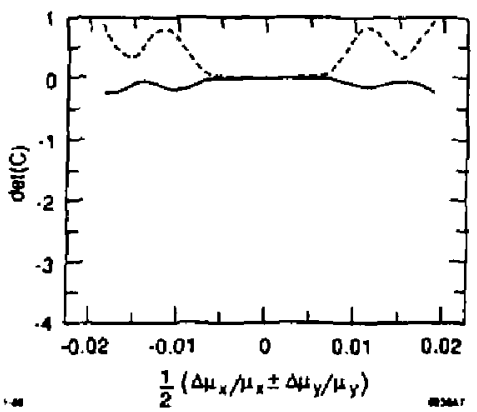

Fig. 7 det $C$ as a function of systematic phase-advance errors of equal sign (dashed line) and of opposite sign (solid line) in the entire North Arc with tapered roll trantitions.

In the modified Nortb Arc, and in the cuse of equal emittances in botb planes (for whict cross-plane coupling with 0 < det $C<1$ is benign), this particular tolerance is very broad and one expects the seovitivity to ertors to be comparable to that of a Bat Arc. For unequal $x$ and $y$ emittances, the improvemeds are, bowever, nat expected to be st grest fsee Fig. 7 - dashed line).

In practice, the system can be perturbed by both random and systematic erroes. Also, the Lolerance to errors depends on the requirement pul or the phase-space at the Arr exit. As was mentioned in the introduction, the cross-plane coupling distortions of the phase-space are correctable in the Final 
Focus, but large distortions of the bean envelope - from any kind of error - can result in unacceptable background in the experiment, and must therefore be svoided. This leads us to define a colerance in terms of the maximum deviation from the gominal size reached by the beam eavelope at the end of the Arss, as generated through the mixing of the distortiong from both random and systematic errors. Extensive computer simulations ${ }^{15.16 .19}$ were performed to evaluate the sensitivity of the Arcs, witb both tandom and systematic ertors. It was found that in the case of equal emit tances, the Atcs with modified roll traositions are about as seasitive to errors as a fat Arc without rolls. We Illustrate this point wijt the result from one of these simulations in Figs. 8(a)-(c), where the geometric mean of the maximum growth of the horizontal and vertical monochramatic bearn sizes at the end of the Arcs is calculated for the ariginal Nortb Arc. for the tapered Nortb Arc, and for a hypotbetical flat Arc. ${ }^{\text {s }}$ The errors are the same in each case and carrespond to cyatematic erm-s of 001 and to a sample of randomly distributed errors with a standard deviation of 0.005 , in botb the focusing and the defocusing magnets. As can be seen. the distortions are amost the same for the tapered Arr [case (b)] and for the flat Arc [case (c)]. Similar results were obtained for the South Arc.

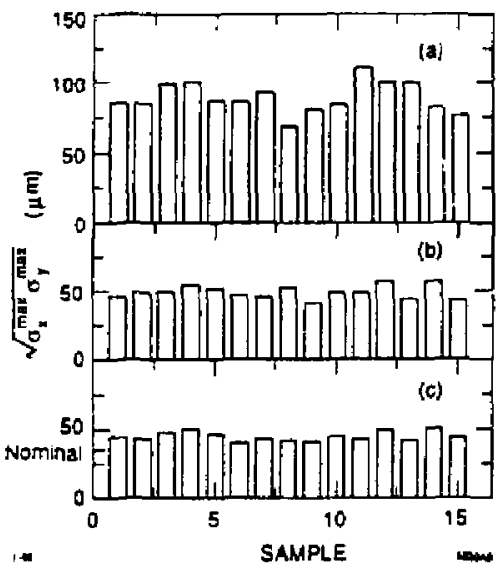

Figs. $8(a),(b)$ and (c). Marimum growth of the geometric mean of the monothromatic beam sizes in the vertical and horianial planes at the end of the North Are perturted by systematic errors with the same sign in each plane, of 0.01 , and nandom errors. with a standard detiation of 0.005 . The oame sample of errory are tued for camparing the Narth Ancs wish the onginal roll transitions (a), with the tapered roll transitions (b), and without rolls (c). The nominal valre is $35 \mu \mathrm{m}$ in each plane.

\section{OPTIMIZATION OF TAPERED ROLL TRANSITION}

A perfect malch of the betatron transfer can be achieved by including at least five cells in the transition. but is not practical because the siga of the rolls must alternate in this case (presumably because the phase-advance actoss more than iwo cells becomes larger $($ han $x$ ), and because the solution depends in this case very nonlineariy on the cotal roll of the transition.

The distribution of rolls indicated in Fig $3(b)$, however, can be improved. An example of such an optimuation js shown in Fig 9, where the maximum positive and negative values of del $C$, occuring when the phase-advances between tbe pairs of roll transitions shown in Fig. 3 are perturbed to satisfy $\left(1 / 2 \mu 13 \mu_{x} \pm 3 \mu_{y}\right)= \pm 90^{\circ}$. is computed as a function of $r$. The dependarce is fairly qat. The optimum value of $r=028$ results in sligh: improvements in the overall performance. ${ }^{14}$

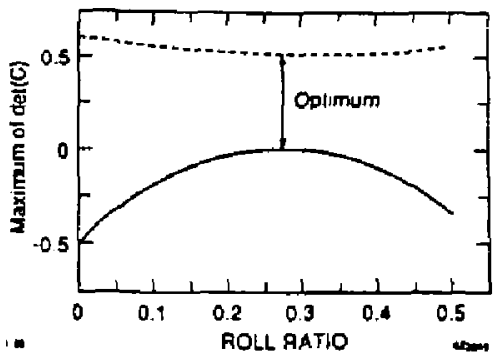

Fig. 9. Maximum value of det C resulting from systematic emors with opposite sign (dashed line) and with the same stgn (colid (inc) between the tapered irassitsong of Fig. S(b), as a funetion of the noll rotio 5 .
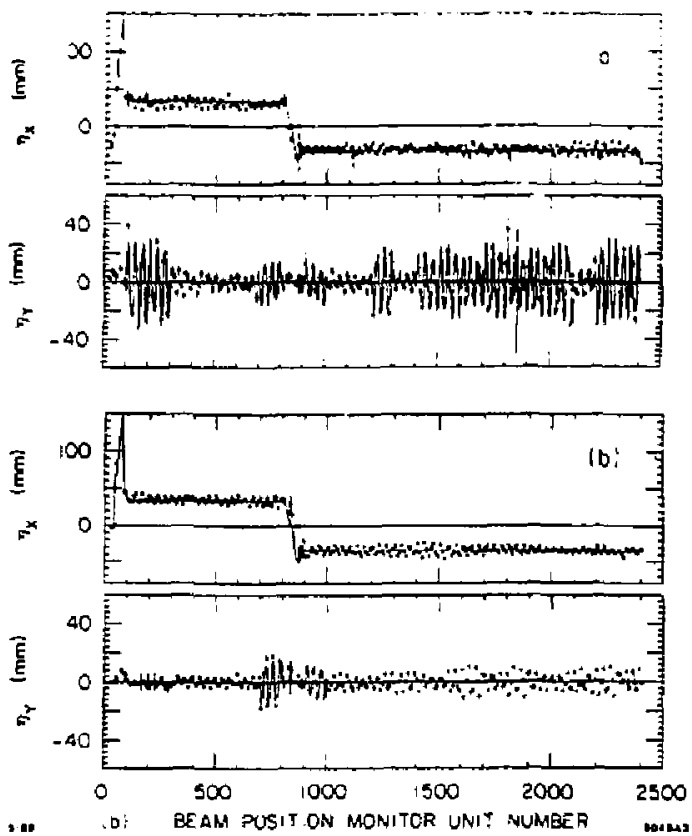

Figs. $10(a)$ and (b). Harizontal and pertical dispersion meostred in the North Arc, before (a) and after (b) the installation of the capered roll tmansitions. The vertical dispersion wat essentially suppressed by the madification.

\section{PERFORMANCE AND CONCLUDING REMARKS}

The performance of the modification wa particularly clear for the suppression of vertical lattice dispersion. This can be seen from the mearurements in the North Arc, befote und after the modification of the roll transitions [uee Figs. 10(a) and (b)].

The minimization of the coupling in the betatron transfer which was achieved during the recommissioning of the Accs after the instalation of the modification canool be attributed solely to this design change. It was also the result of the previous phaseadvance adjustmenta, of several other empirical adjusiments and ratering performed at turn-oD.

The modification did in addition improve the overall per. formance of the Atc, by reducing. as expected, the need for 
feeding back on the optic to cuncel variations lrom teering or other changes. However, later, expectations on the quality of the phase-apace at the exit to the Arcs were also enhanced, in particular from the sequiremeat to minimize beam tail induced backgrounds in the detectos. ${ }^{7}$ Becaune of this it became neces. ary to implemeot further and more procise correctiona, ']

More receatly, it ha been pasible to determine the transfer matrix along the Arc bean-line, by fitting betatron oacillationa launched at wever in in put phaves." Such ealculations have abown that prevencly det $\mathrm{C} \cong 0.2$ at the exit to the North Arc, lesding to small coupling eftects in the cure of equal emittances.

\section{APPENDXX}

It is possibie to calculate the projected emittances exactly with the implifying anumption of upright phese-ellipees and of beam initially uncoupted in both planew, by tolding the contributions to each emitinec projection from the two plenes. Beceuve the phave-ellipaes are nol in general upright. the derived expresion is an upper bound of the caees with phuse-ellipses of arbitrary orientation. Ooe obtains:

$$
c_{x, D}=\left[\epsilon_{x, p}(0)|l-\operatorname{det} C|+\epsilon_{y, x}(0)|\operatorname{det} C|\right] \sqrt{1+\mathcal{F}},
$$

where $c_{3}(0)$ and $e_{0}(0)$ are the initial values, and where the factor:

$$
F=\frac{c_{x}(0) c_{1}(0)|\operatorname{det} C \||-\operatorname{det} C \mid}{\epsilon_{\pi, x}(0)|1-\operatorname{det} C|+c_{n, x}(0)|\operatorname{det} C|}\left(\frac{\lambda_{z}^{2}}{\lambda_{1}^{2}}+\frac{\lambda_{2}^{2}}{\lambda_{x}^{2}}-2\right)
$$

describes the mixing which results from folding the phase-ellipwes if they are not aimilu. Such divimilarity aries from upright quadrupole perturbations to the lnttice, both through random and, in the presence of rolh, systemulic etratu. The parameter $\lambda_{x, y}$ describe the twonit ude of the mitmuch which result in each plane. These parameters are defined in rormalized phave-opece a the ratio of the radius of the circle in which a distorted phace ellipae is inscribed to that of the smaller circle which correspands to the malched case. ${ }^{12}$ If tbere are ao upright quadrupole earora $\left(\lambda_{x, y}=1\right)$ or if the two phave-ellipes are similar $\left(\lambda_{*}=\lambda_{p}\right)$. then the mixing term $F=0$. For minmstehe with $\lambda_{1,} \delta 2$, correaponding to upright quadrupole error of up to $1 \%$, then $\sqrt{1+f} \leqslant 2.5$. For wuch canes, the inequality tign in the expresion given in Eq. (2) can be replaced by an equality sigo. for approximale calculation.

\section{ACKNOWLEDGEMENTS}

The ensitivity of the Are beam transfer to syntematic error was a central inue in the commiegjoning of the SLC during the nummer of 1987. At that time, large number of people contributed to underatending and to solving thene problems, each one trom bis or bet point of view. In particular. we would like to alkowledge the work and enthusiasm of $F$. Bulus, D. Burke, J. Haisninaki, J. Murray, N. Phinney, M. Plachd. L. Bivkin, J. Stepperd and R. Stieuing.

Lat, but not leant, we would like to thank the Mechanical Engineering and SLC Alignment Groupg for the very rigorous and efficient installation and aligament wotk related to the initial set-up of the Aros and to this tapered "rollfix."

\section{REFERENCES}

1. S. Kbeifets et al., "Beam Optical Deaigo and Studies of the SLC Arca," SLAC-PUB-4013 (1987).

2. K. L. Brown, "A Second-Order Magaetic Optical Achromat," SLAC-PUB-2257 (1979).

3. T. H. Fiegutb et al., "Studies of Anomalous Dispersion in tbe SLC Second Order Acbromats," SLAC-PLB-\$096 (1987).

4. M. Sands, "Betatron Oraillations and Rolled Achromat," SLAC-CN-355 (1987).

5. J. J. Murray, private communication (1984).

6. P. S. Bambude, "Benm Dynamics in the SLC Final Focus System," SLAC-PUB - 227 (June 1987).

7. D. L. Burke el al. "Bean Collimation and Detector Backgrounds at $e^{+} e^{-}$Lives Colliders," these proceedings.

8. J. Haissingki et al. "A Technique for Correcting the Betatron Tunes of the SLC Arcs," theae proceedings.

9. E. D. Courtent and H. S. Snyder, "Theory of the Alternating Gradient Synchrotron," Ann. of Phys. 3, 1-48 (1958).

10. K. L. Brown and R. V. Servranclk, "Croas-Plane Coupling and its Effects on Projected Emitlances," SLAC-PLB4679 (July 1988).

11. P. S. Bumbede and A. Hutton, "Specification of Harmonic Correction: (Wirefix) for the SLC Arcs," in preparation.

12. P. S. Bambade, "Betatron Pbase-Space Diagnostic in a FODO Array," SLAC-CN-367 (October 1988).

13. See dro: T. H. Fieguth et al. "Tuning the Arcs of the SLAC Linear Collider," SLAC-PUB-4528 (May 1988).

14. This was the reault of work by M. Sands and, independently, L. Rivkin.

15. D. M. Riteon, ARCSIM, privale program.

16. R. V. Servraitelex and K. L. Brown, DIMAD program; SLAC-REP-285 (May 1985).

17. J. J. Murray and T. Fiegutb, MURTLE, private program.

18. T. L. Barklow, "A Technique for Measuring the $4 \times 4$ Tranofer Matrix for Beamline Sections with Crosg-Plane Coupling," these proceedings. 
VI.s "Speciflcation of Harmonic Corrections (Wirefix) for the SLC Arcs"

This collider note describes the theory and the design and implementation of barmonic corrections in the arcs. Normally a method which applies to circular accelerators, harmonic corrections can under certain conditions be used efficiently for doing optical corrections in long open FODO arrays. Such corrections were introduced in the last third of the arcs to supplement the optical corrections in the final focus. They are necessary to enable adjusting the beam at the entrance to the final focus, where a nearly matched phase-space is requirs: $\dot{d}$ in minimize backgrounds in the experimental apparatus. 


\title{
SINGLE PASS COLLIDER MEMO CN-370
}

\author{
AUTHOR: Philip Bambade, Andrew Hutton \\ DATE: February 1, 1989 \\ TITLE: \\ SPECIFICATION OF HARMONIC CORRECTIONS (WIREFIX) \\ FOR THE SLC ARCS**
}

\section{INTRODUCTION}

In the original SLC commissioning plans, it was thought that accumulated optical mismatch, generated by focusing errors in the whole machine, would be corrected at the very end, in the Final Focus. Dedicated correctors for optical matching and a special adjustment strategy were planned for this purpose, with a large tuning range of up to about a factor four in any dimension of the beam phase-spacel.

For several reasons, this does not appear to be feasible. One major constraint limiting the magnitude of mismatches which cas be absorbed in the Final Focus is the background generated in the detector, from electromagnetic debris and from muons produced when beam-tails strike apertures there. The apertures in the Final Focus, normalized to the nominal beam size, are in effect significantily smaller than in the Arcs, both upstream and downstream of the dedicated optical correctors. Because of this, otherwise correctable optical distortions can result in enhanced backgrounds, as imperfectly collimated beam tails get magnified by the optical distortions, and can get scraped off.

With the present collimation and shielding arrangements, it is necessary to control the bearn upstream of the Final Focus in order to inject a nearly matched phuse-space there. Following work by Stiening in the Linac ${ }^{2}$, and by Fieguth in the $\mathrm{Arcs}^{3}$, we have developed and installed a new system of harmonic focusing corrections at the end of the SLC Arcs, to provide such control.

* The protection collimator $\mathrm{PC} 18$, for example, has a radius of 4 millimeters. After normal. izing by the nominal beam size, it is smaller than the Arc 6 millimeter vacuum chamber by a lactor 6.7 .

* Work supported by the Department of Energy Contract DE-AC03$765 F 00515$. 
The scheme consists of introducing smi'i regular and skew focusing deviations at specific harmonics of the betatron frequency which the phase-space is specially sensitive to. The harmonics in question are the zeroeth harmonic and the second barmonic of the betatron frequency". The focusing deviations are introduced in the Arc lattice by perturbing the strengths of the combined function magnets with a set of appropriately rewired trim windings at their backleg ${ }^{\dagger}$. The corrections provide an efficient way for adjusting both for errors in the Arc lattice and for mismatch at the injection to the Arc, generated by the upstream systems.

In this note, we describe the specification of this correction procedure as well as the present installation. Initial operational experience with this new method for adjusting bearn-lines is presented elsewheret.

We begin with a description of the theoretical work which grided the specification. We then define the harmonics in the case of the Arc lattice, and describe the wiring modification and the strength of the regular and skew quadrupole components which can be generated, as calculated with POISSON ${ }^{5}$. We also describe the intra-magnet wiring arrangement through which the spatial strength modulations are produced.

Finally we present the predicted effects from the present installation, and outline possible improvements.

* In a circular accelerator, these harmonics cortespond to the half-integer resonance in each plane, and to the sum and difference coupling resonances.

t The backleg windings were introduced in the Arc magnets originally to provide a step-wise adaptation of the strength of the lattice to the energy of the beam, which loses about $1 \mathrm{Gev}$ through the emission of synchrotron radiation in the guide-field. 


\section{THEORY OF HARMONIC CORRECTIONS}

\section{1 Concept of Harmonic Correction in a Beam-Line $e^{2,3}$}

In a beam-line where the focusing lattice consists of a periodic FODO array, the optical mismatch which occurs from focusing errors is conveniently described by an ellipse which rotates in phase-space with the betatron phase-advance ${ }^{6}$. Because ellipses are invariant under rotation of $\pi$, the beats in the beam envelope occur at twice the betatron frequency. Therefore, focusing errors which are separated by $\pi$, and more generally, which occur at twice the betatron frequency, wi'l build up and enhance the optical mismatch.

Thus it is natural to consider adjusting the lattice and the phase-space in a FODO array by introducing controllable focusing perturbations at twice the betatron frequency. In general, the focusing errors in tie lattice are random. Such focusing perturbations will thus add to or subtract from the strength of the Fourier component of the random errors which is at twice the betatrun frequency, Controlling the strength of this harmonic thus enables to ther make an overall correction of the lattice, or to purposely distort the lattice to minimize optical mismatch in the injected beam. This notion can be applied both to regular focusing errors and to skew focusing errors.

Because the perturbations from the errors are random and contain in generai a systematic component, the accumulating optical mismatch will not remain indefinitely in phase with the focusing perturbations which are introduced. The longer the array, the larger the phase-shift between the two will be, and the weaker the effects from the controls become as the system becomes more narrow-band. Because of this, 'larmonic corrections cannot be performed over a region that is too long, without losiag much of their efficiency.

A second reason why harmonic corrections can in general not be applied over a region that is too long arises if the momentuin dependance of the focusing, referred to as the chromaticity of the lattice, is not or is imperfectly corrected. In this case, the finite momentum spread in the beam will cause the optical mismatch from the focr-ing deviations to filament into a larger effective phase-space. The efficiency of a harmonic correction can in this case be reduced. Because the SLC Arcs are designed to be achrumatic, such effects arise only to the extent the chromaticitycorrection, because of the ertors, is imperfect. For the range of etrors which we 
consider ${ }^{\star}$, this is a small effect which we will not consider here.

\section{II.2 Scope of Theory}

The scope of this theoretical description is not that of full geterality or rigor. The goal is rather to show the basic features of betatron oscillations and of transverse phase-space, as they are inaged through a FODO array which has been perturbed by a periodical focusing deviation. We will calculate the effects for each perturbation separately, in an idealized system with no errors, and ignore mixing effects which arise when several perturbations are applied simultaneously. Such mixing effects can change the magnitude of the effects, but do not change the basic features of the solutions which we will derive.

The only case of mixing which we will treat is that of a systematic focusing deviation, from the random errors or applied as an independent perturbation, combined with a periodic focusing perturbation at twice the betatron frequency. Rather than calculating the explicit solution for this case, we will indicate the magnitude of the reduction factor which results.

A practical case of periodic focusing deviation applied to a lattice with random errors will be explored through simulation in section VI.

In order to calculate the effects to be expected from harmonic focusing perturbations in a repetitive lattice, it is convenient to introduce and work with normalized variables.

\section{3 Normalized Variables ${ }^{7}$}

The transverse motion of a particle in a focusing array is governed by Hill's equation:

$$
\ddot{z}+K z=0
$$

where $z=x, y$ and $s=c t$ are the transvers and longitudiral coordinates respectively, and where $K$ represents the strength of the varying restoring force from the focusing array.

\footnotetext{
* We consider focusing pertarbations of up to about one percent.
} 
The use of the normalized variables $u_{x, y}=\frac{x}{\sqrt{\phi}}$ and $d \phi=\frac{d y}{f}$, where $\beta$ satisfies:

$$
\frac{1}{2} \beta \ddot{\beta}-\frac{1}{4} \dot{\beta}^{2}+K \beta^{2}-1=0,
$$

transforms (1) into the equation of a pure harmonic oscillator:

$$
\ddot{u}_{z, y}+u_{x, y}=0 \text {. }
$$

In (1) and (2), $\dot{f} \equiv \frac{d f}{d s}$, where $f=z, \beta$. In $(3), \dot{u} \equiv \frac{d u_{x, y}}{d \phi}$. The solution of $(1)$ is thus:

$$
z=a \sqrt{\beta} \cos (\phi+b),
$$

whure $a$ and $b$ are integration constants, and is referred to as a betatron oscillation. By definition the frequency of this oscillation, written as a function of the phase variable $\phi$, is one.

Althougli the form in (4) is general, it is specially sujted to periodic arrays consisting of repeated cells. In this case $K, \beta$ and $\phi$ are periodic with the cell length and the betatron oscillation is pseudo-harmonic. An equivalent harmonic oscillator can be defined by sampling (4) at each cell;

$$
\left\{\begin{array}{l}
z_{n}=a \sqrt{\beta} \cos \left(\phi_{n}+b\right) \\
\text { with } \phi_{n}=n \mu
\end{array}\right.
$$

where $\mu$ is the phase-shift per ceil. In what follows, we consider perturbations of (3). The solutions we will derive coincide with those of (1) at the sampling points, after rescaling by $\sqrt{ } \beta$. It is therefore possible to use (1) or (3) interchangeably, as long as one considers the restriction of the solution to the sampling points. We will use this fact to write simplified expressions for the perturbed motion.

\section{II.4 Periodical Focusing Perturbations}

\section{I1.4.1 Regular Quadrupole Perturbations}

* From the sampling theorem, (4) is not undersampled by this procedure as long as the cell phase-shift is less than $\pi$, which is always the case ${ }^{3}$. In the SLC Arcs, $\mu=\frac{3 \pi}{5}$. 
To study the effect from a focusing modulation with regular quadrupoles, we replace $K$ by $K+k \cos (\nu \phi+\psi)$ in the governing equation $(1$,$) , where k \cos (\nu \phi+\dot{\psi})$ represents a periodic deviation in the restoring force $K$, of amplitude $k$ and of phase $\psi$, and with $\nu$ cycles per radians of phase-advance along the array. For the horizontal motion $(z=x)$, the most important deviations are the ones in the focusing lenses. Conversely, for the vertical motion $(z=y)$, the most important deviations are the ones in the defocusing lenses ". We consider smail errors so hiat $k \ll K$.

In the normalized system, the equation of motion in (3) becomes:

$$
\bar{u}_{x, y}+\left(1+g^{r} \cos \left(\nu \phi+\psi^{r}\right)\right) u_{x, y}=0,
$$

with $g^{r}=\beta^{2} k$, and $\dot{\psi}^{r}=\psi$. The function $\beta$ is sampled at the center of each focusing magnet for the horizontal motion, and at the center of each defocusing magnet for the vertical motion. Since only errors in the focusing (respectively defocusing) magnets affect the motion significastly in the horizontal (respectively vertical) planes, the factor $g^{r}$ can be considered constants in (6).

\section{IJ.4.2 Skew Quadrupole Perturbations}

Similarly, we write the equations which govern the motion when focusing modulations with skew quadrupoles are applied. In this case, the restoring focusing force, proportional to the beam excursion in each transverse plane, acts on the perpendicule.i plane. This generates cross-plane coupling. The equations of motion are in this case:

$$
\left\{\begin{array}{l}
\ddot{u}_{x}+u_{z}+g^{s} \cos \left(\nu \phi+\psi^{s}\right) u_{y}=0 \\
\ddot{u}_{y}+u_{y}+g^{s} \cos \left(\nu \phi+\psi^{s}\right) u_{x}=0,
\end{array}\right.
$$

where $g^{s}$ and $\psi^{s}$ represent the am-plitude and phase of the skew focusing modulation

- This results from the fact that in a FODO array, the beam size is naturully larger in each lens, in the plane in which the lens focuses. Because of this, the set of $F$ and $D$ magnets form elose to orthogonal sets, ia terms of their effect on the one dimensional beam motion. A measure of this orthogonality is given by the ratio of the maximum to minimum beam size in the array. In the Are lattice, this ratio is 2.8 . The two sets are orthogonal for all practical purposes. 
along the array ${ }^{\dagger}$.

Defining $u_{ \pm}=u_{x} \pm u_{y}$, we can rewrite (7) in a form similar to (6):

$$
\ddot{u}_{ \pm}+\left(1 \pm g^{3} \cos \left(\nu \phi+\psi^{\prime}\right)\right) u_{ \pm}=0
$$

Next, we show that the perturbed motions, solutions of (6) and (7), are affected significantly only for two specific values of the frequency $v$ of the modulations, namely:

$$
\nu \simeq 0 \text { and } \nu \simeq 2 \text {, }
$$

corresponding respectively to the systematic component in the errors and to the secor! harmonic of the betatron frequency.

\section{II.5 First Order Solution}

\section{II.5.I Method of Variation of Constants}

Following Nayfeh, and along with the method of variation of constants, we

t The factor $g^{3}$ can be related to the amplitude of skew quadrupole modulations in the focusing and in the defocusing rnagnets as follows:

$$
g_{D, F}^{d}=\beta_{\varepsilon, y}^{t} \beta_{y, x}^{b} k_{D, F},
$$

where $k_{D}$, r represent deviations to the focusing ind defocusing magnet strengths in the regular coordinate system. The largest effect in the first (respectively second) of the two equations in ( 7 ) occurs in the defocusing (respectively focusing) magnet, since $u_{y}$ (respectively $u_{r}$ ) is naturally the largest there. Therefore the term $g^{a}$ in the first (tespectively secand) equation in (7) is essentially $g_{D}^{\prime}$ (respectively $g_{F}^{\prime}$ ). Since in a FODO array, $\beta_{t, y}^{F}=\beta_{y, x}^{D}$, we have $g_{D}^{\prime}=g_{F}^{\prime}$. For this reason, we use the same $g^{\prime}$ in the two equations in (7).

Furthermore, it has been shown (through computer simulation) that modulations where the skew quadrupole components bave opposite signs in the focusing and in the defocusing lenses produce effects which cancel over one betatron periad, except in the case of the systematic component, corresponding to $\nu \simeq 0$. For this reason, we use the same phase $\psi^{\prime \prime}$

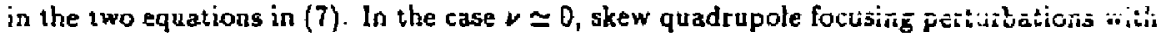
opposite sign in the focusing and in the defocusing magnets correspond to a rotation of the coordinate systern. It can be shown that such a rotation can be expressed in the normalized variables by simply exchanging the sign of $g^{3}$ in one of the two equations in $(7)^{9}$. In this case, the two equations cannot be decoupled simply as in (8). 
solve (6) and (7) by searching for solutions of the form ${ }^{10}$ :

$$
u=a \cos (\phi+\varphi)) .
$$

where $a$ and $\varphi$ are functions of $\phi$ to be determined. In (10) and in the rest of this paragraph, $u$ represents $u_{z, y,+,-}$. By taking the first derivative of (10), letting a and $\phi$ vary, and by requiring that the result be what it would be if $a$ and $\phi$ were constants (i.e. $\dot{u}=-n \sin (\phi+\varphi)$ ), an equation relating the first order derivatives $\dot{a}$ and $\dot{\phi}$ is found. By calculating the second derivative of $u$, and after inserting it in an equation of the form of $(6)$, we find a second equation relating $a$ and $\dot{\varphi}$. Thus we have replaced a second order differential equation in $u$ by two coupled first order differential equations in $a$ and in $\varphi$. By solving this coupled system, we find that $a$ and $\varphi$ satisfy:

$$
\begin{aligned}
\frac{d a}{a} & =\frac{g}{4} d \phi \sin [(2-\nu) \phi+2 \varphi-\psi] \\
& +\sin [(2+\nu) \phi+2 \varphi+\psi], \\
d \varphi & =\frac{g}{4} d \phi(2 \cos [\nu \phi+\psi] \\
& +\cos [(2-\nu) \phi+2 \varphi-\psi] \\
& +\cos [(2+\nu) \phi+2 \varphi+\psi]),
\end{aligned}
$$

where $\psi=\psi^{r, s}$ and $g=g^{r, s}$.

\section{II.5.2 A veraging Method}

To find the behavior of the solutions in the limit of small $g \phi$, we solve (11) to first order. To do so, we first note the fact that for values of $\nu \neq 0,2$, the solutions of (11) are rapidly oscillating functions with amplitudes of order $g$. We will neglect such contributions, as they are bounded by $g$. Thus for $\nu \neq 0,2$, the motion, solution of (6) and (7), is perturbed negligibly.

The amplitudes of the functions $a$ and $\varphi$ cas only become significant if the functions on the right hand side of (11) are slowly varying functions. This occurs for $\nu \simeq 0,2$.

From now on, we will write go for the magnitude of the systematic focusing deviations, corresponding to $\nu \simeq 0_{1}$ and $g_{2}$ and $\psi_{2}$ for the amplitude and phase of focusing modulations at twice the betatron frequency, corresponding to $\nu \simeq 2$. 
We first solve for $\nu \simeq 0$. The solution is obtained by integration of (11b). This gives:

$$
u \simeq a_{0} \cos \left(\left\{1+\frac{g_{0}}{2}\right) \phi+\varphi_{0}\right\}
$$

where $a_{0}$ and $\varphi_{0}$ are integration constants.

Next we solve for $\nu \simeq 2$, integrating this time both (11a) and (11b). From (1Ib), and including only the slowly varying term $\cos \left[(2-\nu) \phi+2 \varphi-\psi_{2}\right]$, we note that $d \varphi \leq \frac{1}{4} g_{2} d \phi$. The total variation of $\varphi$ over the interval of integration is thus bounded by $\frac{1}{4} g_{2} \phi$. Thus the right hand sides of (11) stay about constant if $\frac{1}{4} g_{2} \phi \ll \pi$. We can in this case treat the slowly varying terms on the right hand side of (11) as constants in the integration. We obtain in this case:

$$
u \approx I_{0} \mathrm{e}^{\lambda \phi} \cos \left[(1+\kappa) \phi+\varphi_{0}\right] .
$$

where:

$$
\left\{\begin{array}{l}
\lambda=\frac{2}{4} \sin \left(2 \varphi_{0}-\psi_{2}\right) \\
\kappa=\frac{\phi_{2}}{4} \cos \left(2 \varphi_{0}-\psi_{2}\right)
\end{array}\right.
$$

\section{II.6 Physical Description of Perturbed Motion}

\section{II.6. I One-Dimensional Oscillations}

From (14), (15) and (16), we characterize the effect of segular focusing modulations on the one-dimensional motion, in the limit of small perturbation, as follows: Case $\nu \simeq 0$ :

The fundamental frequency of the oscillations is shifted by systematic regular focusing deviations, corresponding to $\nu=0$. This shift is indepesdent of the initial phase of the betatron oscillation.

Case $\nu \simeq 2$ :

The amplitude and frequency of the oscillations are perturbed resonantly by regular focusing modulations at the second harmonic of the fundamental betatron frequency, corsesponding to $\nu=2$. Depending on the phase $\psi_{2}$ of this modulation and on the initial phase $\varphi_{0}$ of the betatron oscillation, the amplitude will initially decay or grow exponentially with a growth rate $\lambda_{\text {, and the frequency will increase }}$ 
or decrease linearly by the slippage parameter $\kappa$. These two effects are out of phase: maximum frequency-slippage coincides with a constant amplitude, and zero frequency-slippage with a maximally perturbed amplitude".

We will describe the consequences for the phase-space and for the beam envelope in the next section.

\section{II.6.2 Two-Dimensional Oscillations}

Case $\nu \simeq 0:$ (Systematic Skew Component)

We can use (12) and $u_{ \pm}=u_{x} \pm u_{y}$ to calculate the effects from systematic skew perturbation. We obtain:

$$
\begin{aligned}
u_{x} \simeq\left[u_{x}(0) \cos \phi\right. & \left.+\dot{u}_{x}(0) \sin \phi\right] \cos g_{0}^{5} \phi \\
& +\left[-u_{y}(0) \sin \phi+\dot{u}_{y}(0) \cos \phi\right] \sin g_{0}^{5} \phi
\end{aligned}
$$

and:

$$
\begin{aligned}
u_{y} \simeq\left[-u_{x}(0) \sin \phi\right. & \left.+\dot{u}_{x}(0) \cos \phi\right] \sin g_{0}^{j} \phi \\
& +\left[u_{y}(0) \cos \phi+\dot{u}_{y}(0) \sin \phi\right] \cos g_{0}^{A} \phi
\end{aligned}
$$

The results in (15a) and (15b) show that in the case of a systematic skew focusing perturbation ( $\nu \simeq 0$ ), oscillations originating in one pläic are gradually transferred into the other plane, and that the sum of squares of the oscillation amplitudes in both planes remains constant. Thus there is beating between the two planes. The period of this beating is determined by $g$. This is true for both the positions $u_{x, y}$ and the angles $\dot{u}_{x, y}$, as can be seen from differentiating (15). Therefore the beating phenomenon which arises is between the two full phasespaces in both planes.

\section{Case $\nu \simeq 0$ : (Coordinzte Rotation)}

The solutions are in this case simple harmonic oscillators, in the rotated coordinate system. We will not write these solutions explicitly.

\section{Case $\nu \simeq 2$ :}

* The separation between the two initial phases $\varphi_{0}$, corresponding to maximum growth or decay, and to maximum phase-slippage, depends on the magaitude of the perturbation. It is strictly $45 \mathrm{deg}$ only in the limit of small perturbation. We will describe the behavior for larger perturbation in the next section. 
From (8) and (i3), the form of the solutions are:

$$
u_{ \pm}=a_{0}^{ \pm} \mathrm{e}^{\lambda \pm \phi} \cos \left[\left(1 \pm \kappa_{ \pm} \phi+\varphi_{0}^{ \pm}\right]\right.
$$

For initial conditions contained in one of two $u_{x}, \dot{u}_{x}$ or $u_{y}, \dot{u}_{y}$ planes, corresponding to the propagation of betatron oscillations launched from one plane at a time (for example: $u_{y}(0)=\dot{u}_{y}(0)=0$, one can show that the solutions can be written as the sum of two functions, one exponentially growing and one exponentially decaying:

$$
u_{x, y}=\frac{a_{0}}{2}\left[e^{\lambda \phi} \cos \left[(1+\kappa) \phi+\varphi_{0}\right] \pm e^{-\lambda \phi} \cos \left[(1-\kappa) \phi+\varphi_{0}\right]\right\}
$$

In this case, the oscillations will grow exponentially in both planes for arbitra:y initial phase.

It is however possible to find initial conditions such that both $u_{+}$and $u_{-}$ decay simultaneously initially. This has been stown independently by solving (8) numerically ${ }^{9}$. One example of such initial conditions is $u_{x}\{0\}=\dot{u}_{x}(0)=u_{y}(0)=$ $\dot{u}_{y}(0)$.

In the next section, we will analyse the consequences from this cross-plane coupling on the areas of the beam phase-space, projected in each plane (projected emittances), and for the beam envelopes, in each of the above cases.

\section{II.7 Physical Description of Perturbed Phase-Space}

\section{II.7.1 Matched Phase-Space}

In the case of a perfect lattice and when the beam phase-space is matched at the input, it remains matched as it is imaged through the array by the optics.

The equation of the envelope of the matched phase-space is easily constructed from the form of the betatron oscillation in (4). One obtains an ellipse:

$$
\gamma z^{2}+2 \alpha z \dot{z}+\beta \dot{z}^{2}=a^{2}
$$

where $\dot{z}=\frac{d x}{d s}, \gamma=\frac{1+\frac{e^{2}}{d}}{s}$, and $\alpha=\frac{-\dot{d}}{2}$. The quantity in (18a) is called the Courant- 
Snyder invariant ${ }^{7}$. It can also be written in matrix form:

$$
(z, \dot{z}) \mathrm{T}^{-1}\left(\begin{array}{l}
z \\
\dot{z}
\end{array}\right)=\alpha^{2}, \text { where } \mathrm{T}=\left(\begin{array}{cc}
\beta & -\alpha \\
-\alpha & \gamma
\end{array}\right) \text { and } \operatorname{det}(\mathrm{T})=1
$$

The equations in (18) also define the closed phase-space trajectory of a particle with initial condition $a$. The parameters $\alpha, \beta$ and $\gamma$ are called Twiss parameters. They characterize the lattice. They also describe the beam phase-space if and when it is matched to the lattice. In this case, the beam-matrix ${ }^{11} \sigma=\epsilon \mathrm{T}$. The area $\pi a^{2}$ of the ellipse is identified as $\pi$ times the emittance $e$.

In the normalized coordinates $u=\frac{\xi}{\sqrt{\beta}}$ and $\dot{u}=\frac{d u}{d \phi}=\sqrt{\beta}\left(\dot{z}-\frac{\dot{\beta}}{2 \beta} z\right)$ defined in II.3, the matched phase-space is a circle of radius $\epsilon$. This can be verified by direct substitution in (18a).

II.7.2 Perturbed One-Dimensional Phase-Space

Case $\nu \simeq 0$ :

In II.6.1, we described the effect of a systematic regular focusing deviation, with maguitude $g_{0}^{r}$, corresponding to $\nu \simeq 0$. The motion is in this case simply frequencyshifted, and the solutions in (12) will satisfy the equation of the matched circle defined in II.7.1. The beam phase-space remains in this case matched".

Case $\nu \cong 2$ :

When the lattice is per . 'rbed by focusing errors, this matched circle is distorted into an ellipse. We descri' * 's condition of the beam phase-space by mismatch. We characterize this misma: $n$ by the ratio $M$ of the radius of the larger circle in which this ellipse is inscribed to that of the initial circle corresponding to the matched case, and by the angle $\phi_{0}$ between its major axis and the abscissa (see Fig. 1): $M$ and $\phi_{0}$ are the amplitude and the phase of the misntatch.

* This is strictly speaking odly approximately true, as the Twiss parameters in (18b), which characterize the FODO array, depend on the phase advance per cell. For example, it can be shown that for a thin lens FODO array'?

$$
\beta_{ \pm}=L \frac{1 \pm \sin (\mu / 2)}{\sin \mu}
$$

whese $\rho_{ \pm}$are the maximum and minimum values, occurring in each plane in the lenses which are focusing, and respectively defocusing, in that plane. For small systematic perturbations of up to one percent, the variation of the Twiss parameters is of the order of one percent. We neglect such variations. 
In 11.6.1, we described the effect of a regular focusing modulation at twice the betatron frequency, with magnitude $g_{2}^{r}$ and with phase $\psi_{2}^{r}$, corresponding to $\nu \simeq 2$. We found that the maximum oscillation amplitude which can be reached is: $u_{\max }=\mathrm{e}^{\frac{5}{4}}$.

The phase $\phi_{0}$ of :ne mismatch depends on how far along the array the mismaich has propagated, i.e. on the accumulated phase advance $\phi$, and on the phase $\psi_{2}^{r}$ of the regular focusin; modulation. We can thus write:

$$
\phi_{0}=\phi+\psi_{2}^{r}
$$

Since to can take an arbitrary value, we can identify:

$$
M=u_{\max }=\mathrm{e}^{\frac{5}{6} \phi}
$$

The equation of the distorted ellipse is calculated in terms of $M$ and $\phi_{0}$, first in the coordinates rotated by $\phi_{0}$ in which it is erect, and then transforming back into the unrotated coordinates. This gives:

$$
\begin{aligned}
u^{2}\left(M^{2} \cos ^{2} \phi_{0}+\frac{1}{M^{2}} \sin ^{2} \phi_{0}\right) & +\dot{u}^{2}\left(M^{2} \sin ^{2} \phi_{0}+\frac{1}{M^{2}} \cos ^{2} \phi_{0}\right) \\
& +2 u \dot{u} \cos \phi_{0} \sin \phi_{0}\left(M^{2}-\frac{1}{M^{2}}\right)=\epsilon .
\end{aligned}
$$

The corresponding beam-matrix, written in the normalized system, in terms of the amplitude $g_{2}^{r}$ and of the phase $\psi_{2}^{r}$, is:

$$
\Sigma_{g_{2}^{*}, \phi_{2}^{*}}(\phi)=\epsilon\left(\begin{array}{ll}
\Sigma_{11} & \Sigma_{12} \\
\Sigma_{12} & \Sigma_{22}
\end{array}\right)
$$

where:

$$
\left\{\begin{array}{l}
\Sigma_{11}=\cosh \left(\frac{q_{2}^{r}}{2} \phi\right)+\sinh \left(\frac{q_{2}^{r}}{2} \phi\right) \cos \left(2 \phi+\psi_{2}^{r}\right) \\
\Sigma_{12}=-\sinh \left(\frac{q_{7}^{r}}{2} \phi\right) \sin \left(2 \phi+\psi_{2}^{r}\right) \\
\Sigma_{22}=\cosh \left(\frac{q_{r}^{r}}{2} \phi\right)-\sinh \left(\frac{q_{2}^{r}}{2} \phi\right) \cos \left(2 \phi+\psi_{2}^{r}\right),
\end{array}\right.
$$


and where $\epsilon$ is the emittance. Transforming back into real coordinates gives:

$$
\left\{\begin{array}{l}
\sigma_{11}=\beta \Sigma_{11} \\
\sigma_{12}=\Sigma_{12}-\alpha \Sigma_{11} \\
\sigma_{22}=\frac{1}{\partial}\left(\Sigma_{22}+\alpha^{2} \Sigma_{11}-2 \alpha \Sigma_{12}\right)
\end{array}\right.
$$

For the ideal lattice, characterized by $g_{2}^{r}=0,(24)$ reduces to $(1 \mathrm{Sb})$ as expected.

In summary, when the lattice is perturbed by a regular focusing modulation at twice the betatron frequency, with amplitude $g_{2}^{\mathrm{T}}$ and phase $\psi_{2}^{\mathrm{T}}$, the phase-space gradually becomes elongated into an ellipse with a major axis which grows exponentially with the accumuiated phase advance, at a growth $\mathrm{rt}_{\mathrm{c}} \frac{i}{2}$. This ellipse rotates in phase-space ac the betatron frequency. The initial value of the phase of the mismatch is determined by the phase $\psi_{2}^{r}$.

From (23), we see that the beam size beats between minimum and maximum values, of $\mathrm{e}^{-\frac{2 \pi}{2} \phi}$ and $\mathrm{e}^{\frac{24}{2} \phi}$ respectively. The seating occurs with a period of $\pi$, as illustrated in Fig. 1. Equation (23) (anc graphically Fig. 1) also allows us to study the behavior of the solutions, both for large and for small perturbation. For small perturbation $\left(g_{2}^{\top} \phi \ll 1\right)$, the first equation in (23) reduces to $\Sigma_{11} \simeq 1+\frac{23 \phi}{2} \cos (2 \phi+$ $\left.4_{2}^{*}\right)$. In this case, the separation in the phase $\phi$ between maximally growing or decaying solutions, and solutions with an unperturbed amplitude (corresponding to $M=11$, is exactly $45 \mathrm{deg}$. This is in agreement with the results from the first order calculations described in I1.6.1. For larger $g_{2}^{+} \phi$, the initial phases corresponding to an unperturbed amplitude move closer and closer to the phase corresponding to a maximally decaying solution. For infinite $g_{2}^{T}$, all solutions become eventually exponentially growing, except for the "single" one for which, strictly, $\cos \left(2 \phi+\psi_{2}^{*}\right)=$ -1 . This has been shown independently by solving (6) numerically".

\section{II.7.3 Coupled Two-Dimensional Phase-Space}

In II.6.2, we gave expressions describing the cross-coupling of betatron oscillations which occurs from systematic skew focusing errors, corresponting to $\nu \simeq 0$. and from skew focusing modulations at twice the betatron freguends, corregnond. ing to $\nu \geq 2$. We use these expressions to infer the evolution of the areas of the phase-space projections in each plane. The correctness of all forms (except (2S) given in this section has been verified through simulation. Results from these simulations are presented in section IV.

$M$ assume an input phase-space where the horizontal and vertical planes are not coupled, but where the emittances are not necessarily equal. Let $r=\epsilon_{y} / \epsilon_{x}$ 
be the ratio of the initial vertical to the initial horizontal emittances (we assume $r \leq 1$ ), and let us normalize the results to the initial horizontal crnittance, by putting $\epsilon_{z}(\phi=0)=1$.

Case $y \simeq 0$ : (Systematic Skew Component)

In this case, it is possible to calculate, from (15), the projected emittances. as the beam is imaged through the array. We obtain:

$$
\left\{\begin{array}{l}
\epsilon_{z}(\phi)=\frac{1}{2}\left[(1+r)+(1-r) \cos g_{0}^{3} \phi\right] \\
\epsilon_{y}(\phi)=\frac{1}{2}\left[(1+r)-(1-r) \cos g_{0}^{\prime} \phi\right] .
\end{array}\right.
$$

Pitting $r=0$ in (25) shows explicitly the beating pheaomenon which we described in II.6.2. One obtains in this case:

$$
\left\{\begin{array}{l}
\epsilon_{x}(\phi)=\cos ^{2}\left(g_{0}^{3} \phi\right) \\
\epsilon_{y}(\phi)=\sin ^{2}\left(g_{0}^{g} \phi\right)
\end{array}\right.
$$

The sum of the two emittaaces is in this case constant, and transverse oscillation energy is transferred back and forth between one plane and the other. This condition describes adequately the imaging of betatron oscillations launched from one plane at a time.

In the special case of equal emittances, corresponding to $r=1$, we have from (25) that:

$$
\epsilon_{x}(\phi)=\epsilon_{y}(\phi)=1
$$

The motion remains in this case unperturbed by systematic skew focusing deviatinns.

For an arbitrary value of $r$, the two emittances will beat between minimum and maximum values of $r$ and 1 . As can be seen, the variations of the two emittances tre out of phase.

\section{Case $\mu \simeq 0: \quad$ (Coordinate Rotation)}

In this case, the projected emittances are calculated by transforming a fourdimensional uncoupled beam-matrix through a rotation of the coordinate svstem. We obtain:

$$
\left\{\begin{array}{l}
\epsilon_{x}(\phi)=\cos ^{2}\left(2 g_{3}^{3}\right)+r \sin ^{2}\left(2 g_{0}^{3}\right) \\
\epsilon_{y}(\phi)=\sin ^{2}\left(2 g_{0}^{3}\right)+r \cos ^{2}\left\{2 g_{0}^{3}\right\} .
\end{array}\right.
$$

The same features apply as for the systematic skew component: for ${ }^{+}=0$, the 
sum of the projected emittances is preserved, and for $r=1$, the phase-space is not perturbed.

\section{Case $v \simeq 2$ :}

In this case, we write, by nudogy with (25):

$$
\left\{\begin{array}{l}
\epsilon_{x}(\phi)=\cosh ^{2} \frac{g^{2}}{4} \phi+r^{2} \sinh ^{2} \frac{q^{2}}{4} \phi \\
\epsilon_{y}(\phi)=\sinh ^{2} \frac{g^{2}}{4} \phi+r^{2} \cosh ^{2} \frac{g^{\prime}}{4} \phi .
\end{array}\right.
$$

In the special case of zero initial emittance in the vertical plane $(r=0)$, we obtain:

$$
\left\{\begin{array}{l}
\epsilon_{x}(\phi)=\cosh ^{2}\left(\frac{\theta^{2}}{4} \phi\right) \\
\epsilon_{3}(\phi)=\sinh ^{2}\left(\frac{g^{2}}{4} \phi\right)
\end{array}\right.
$$

in accordance wich (17), from which all solutions grow exponentially if the initial conditions are restricted to one plane.

The difference between the two emittance projections is in this case constant, and the projected emittances grow exponentially in both planes. This condition descrives adequately the imaging of betatron oscillations launcher from nne nlane a! a time

In the special case of equal initial emittances in each plane $(r=1)$, we cbtain:

$$
\epsilon_{z}(\phi)=\epsilon_{y}(\phi)=\cosh \frac{9_{2}^{3}}{2} \phi
$$

The two projected emittances remain in this case equal, and grow exponentially".

\section{II.8 Summary Description - Number of Independent Perturbations}

Thus we find that, in total, the trangverse motion can be perturbed by ten incependent parsmeters:

* Although the envelope grows, there can exist, as we found in (16), individual solucicas which are decaying. 
1. The one-dimensional horizontal motion can be perturbed in tbree ways, namely through a systematic strength deviation in the focising quadrupoles, and through the amplitude and phase of a periodic focusing deviation at twice the betatron frequency in the focusing quadrupoles.

2. The one-dimensional vectical motion can be perturbed in three ways, namely through a systematic strength deviation in the defocusing quadrupoles, and through the amplitude and phase of a periodic focusing deviation at twice the betation frequency in the defocusing quadrupoles.

3. The two-dimensional coupled motion can be perturbed in four ways, namely through a systematic s'iew quadrupole component, through the amplitude and phase of a periodic shew focusing deviation at twice the betatron frequency ${ }^{\dagger}$, and through an overall coordinate rotation.

These ten perturbations correspond to the number of free parameters in a fully general two-dimensional transfer matrix ${ }^{23}$.

To the extent that the errors are small and that only one perturbation is applied at a time, it is possible to simply parametrize the perturbed motion as a function of these ten farameters, as was shown above. In general, bowever, a full parametrization will be complicated as the mixing between the perturbations, not considered bere, will yield higher order dependances.

In the case of the four-dimensional beam phase-space, it would appear from our calculations that it only can be perturbed in seven indefendent ways, since the two systematic strength deviations, in the focusing and in the defocusing magnets. do not distort the phase-space, and since the coupling effect from the overall coordinate rotation can be reproduced through the systematic skew quadrupole component.

This is however in contradiction with the courting of the number of invariants imposed by Hamiltonian Mechanics. As can be shown, two invariants exist for hamiltonian systems with two degrees of freedom: the volume of the fourdimensional phase-space and the sum of the projections onto each cuordinate plane of any two-dimensional surface in the four-dimensional phase-space ${ }^{14}$. From this counting, one expects eight degrees of freedom for the fully coupled fourdimensional phase-space. We have not resolved this discrepancy.

In the sperial case of equal initial emittances in both planes $(r=1)$, our cai-

i With in bach cases the same sign skew quadrupole component in the focusing and in the defocusing quadrupoles. 
culations show that the fully coupled four-dimensional phase-space has six degrees of freedom. This is in agreement with an independent proof ${ }^{15}$, and has also been verified in extensive computer simulations of optical corrections in the Final Focus System ${ }^{16}$.

\section{II.9 Case of the SLC Arcs}

Not all perturbations are equally important in the case of the SLC Arcs.

Effects from the systematic coordinate rotation can in the SLC be ignored for all practical purposes, because the magnitude of the rotation angle can never be very large: for skew focusing errors of one percent, the rotation angle is only 1.15 degree.

In addition, effects on the phase-space from both the systematic skew quadrupole perturbation and the overall coordinate rotation are vanishing if the beam has, as is nominally specified, equal emittances in both planes.

In the case of the systematic skew quadrupole perturbation, there can however be significant effects on the betatron oscillations and on the transfer matrix. For beams with unequal emittinces, the beats which are produced in each of the two emittances out of phase and are bounded by the larger emittance (see equation (25)). In the case of the SLC, where beams with emittance ratios of about one to three are presently measured at the end of the Linac, this is not a large effect*

Effects from systematic focusing errors, over the whole length of the Arc, in the focusing and in the defocusing quadrupoles, would bo small in entirely flat Ares, except for mixing effects with the other perturbations. In the original design, the Arcs were rolled around their axis to enable following the teriain of the SLAC site, and this generated a strong sensitivity to systematic focusing errors. With the adiabatic roil transition which was introduced to remedy this problem, such systematic errors will produce coupling effects similar to the ories produced by a systematic skew focusing perturbation ${ }^{17}$. In the case of close to equal emittances, the effects on the phase-space from this are small, as was described above, and the modified Arcs have sensitivities which are similar to those of a flat Arc.

* This argument ignores mixing effects between several perturbations. It has in particular been shown that the upper bound described above can be significantly larger if the systematic skew deviation is mixed with a systematic phase difference between the motions in both planes and a one-dimensional mismatch from regular quadrupole errors ${ }^{13}$. 


\section{II.10 Bandwidth Limits}

The effect from the focusing modulation at ihe second harmonic of the betation frequency, corresponding to $\nu=2$, is weakened if a systematic focusing deviation, corresponding to $\nu=0$, is simultaneously present, from either the errors in the array, or applied externally. This results from the gradual phase-shift which accumulates in this case between the resonantly growing optical mismatch and the induced focusing modulation (see Fig. 2). The mixing betwen the two perturbations will cause the resonant growth of the oscillations to rex.ch a maximum and to then decay, in a long-range beating effect. The nomisal phase-space will be fully restored when the phase-shift between the resonantly growing optical mismatch and the induced focusing modulation reaches $\pi$.

We wi]] not derive the explicit form of the perturbed solution. We can however evaluate, at any given point along the array, the reduction in the growth of the oscillation amplitudes, by considering the modulation in frequency domain. The harmonic strength - or spectrum - of the focusing perturbation introduced along the array is given by the modulus of the Fourier transform of the perturbation. Here, we consider periodic perturbations - or modulations - which are applied along an array with finite length, corresponding to a total phase shift of $\phi_{\max }=n \mu$, where $\mu$ is the phase-shift per cell, and $n$ is the number of cells in the array. The harmonic strength is therefore given by:

$$
\begin{aligned}
\left|F\left[d_{n \mu}(\phi) g \mathrm{e}^{i \nu_{\text {perl }} \phi}\right]\right| & =\left|g \delta\left(\nu-\nu_{\text {pert }}\right) \otimes \frac{\sin [n \mu \pi \nu]}{n \mu \pi \nu}\right| \\
& =\left|g \frac{\sin \left[\pi \mu \pi\left(\nu-\nu_{\text {pert }}\right)\right]}{n \mu \pi\left(\nu-\nu_{\text {pert }}\right)}\right|,
\end{aligned}
$$

where $\delta(\nu)$ is the Dirac distribution, symbolizes the convolution product, and $d_{n \mu}(\theta)$ is a function of $\phi$ which is equal to unity between 0 and $n \mu$, and zero everywhere else".

This calculation is illustrat:d in Fig. 3. The range $\Delta v$ over which the harmonic strength is still reasonably large is given by about half the separation between the zeros of the function in (32), or $\Delta \nu= \pm 1 / 2 \pi \mu$ cycles per cadians. This correspords to a maximum phase-shift of $\frac{\pi}{2}$, accumulated between the growing mismatch and

* This funcion is usually called the "door" function. 
the iocusing modulation, along the total length of the array, or to a maximum systematic phase-error per cell of:

$$
\Delta \mu_{\operatorname{nax}}=\frac{\pi}{2 n} .
$$

Equation (33) gives the requirement on systematic errors in the array, as a function of its length, to maintain strong effects from the induced focusing modulation. The system of harmonic focusing corrections installed in the SLC Arcs extends over seven achromats, or $n=70$ cells. The requirement on systematic phase-errors is in this case $\Delta \mu_{\text {max }}= \pm 1.3^{0}$ per cell. 


\section{HARMONIC PERTURBATIONS IN THE SLC ARCS}

\section{1 Definitions}

A systematic perturbation in the focusing or in the defocusing magnets means that each focusing or defocusing magnet is perturbed the same way.

The cell phase-shift in the SLC Arc lattice is $108^{\circ}$ or $\frac{3 \pi}{5}$. Thus a perturbation at twice the betatron frequency is a perturbation whose strength is modulated by $\exp \left(i \frac{6 \pi}{5} k\right)$ along the cells in the array, where $k$ is the cell number.

A total of nine independent barmonic perturbations can be generated in the SLC Arc lattice. We will represent the strengtb perturbations in the focusing and defocusing magnets respectively by $F$ and $D$. We have:

1. Cosine-like in-plane (regular) harizontal second harmonic component:

$$
F(k)=g_{2}^{r} \cos \left(\frac{6 \pi}{5} k\right)
$$

2. Sine-like in-plare (regular) horizontal second harmonic component:

$$
F(k)=g_{2}^{r} \sin \left(\frac{6 \pi}{5} k\right)
$$

3. Cosine-like in-plane (regular) vertical second harmonic component:

$$
D(k)=g_{2}^{r} \cos \left(\frac{6 \pi}{5} k\right)
$$

- Only nine adjustments can be generated, out of the ten which are needed to fully control the optical transfer. The missing one would be a regular focusing perturbation, as in 7 . below, but with the same sign in the focusing and in the defocusing magnets. Such a perturbation can be generated electrically through the harmonic correction system degcribed in this note. or through the aiready installed backleg windings, but has no optical effects because of the achiomaticity of the lattice. The only way in which it can be generated in the SLC lattice is physically moving the focusing magnets closer of fatthet horizontally from the defocusing magnets. 
4. Sine-like in-plane (regular) vertical second barmonic component:

$$
D(k)=g_{2}^{5} \sin \left(\frac{6 \pi}{5} k\right)
$$

5. Cosine-like cross-plane (skew) second barmonic component:

$$
F(k)=D(k)=g_{2}^{3} \cos \left(\frac{6 \pi}{5} k\right)
$$

6. Sine-like cross-plane (skew) second harmonic component:

$$
F(k)=D(k)=g_{2}^{3} \sin \left(\frac{6 \pi}{5} k\right)
$$

7. Systematic regular focusing strength difference between focusing and defocusing magnets (FD-Imbalance):

$$
F(k)=-D(k)=g_{0}^{r}
$$

8. Systematic skew tocusing perturbation in the focusing and defocusing magnets:

$$
F(k)=D(k)=g_{0}^{5}
$$

9. Overall coordinate rotation:

$$
F(k)=-D(k)=g_{0}^{\prime}
$$

\section{2 Strength Perturbations in the Alternating Gradient Magnets}

\section{[I1.2.1 Backleg Wiring Modification}


The combined function magnets in the Arc lattice are equipped with backleg windings on each coil. These backleg windings have 29 turns and are connected in series along one achromat. Their purpose is to provide a step-wise adaptation of the lattice to the energy of the beam, which loses about $1 \mathrm{Gev}$ through the emission of synchrotion radiation in the guide-field. The strengths of each magnet in the $i$ last achrornats afe perturbed individually by separately connecting and powering 4 out of 29 backleg winding turns on the upper and lower coils. The remaining 24 turns" are connected as for the original use of the backleg windings. A schematic of the modified wiring arrangement is shown in Fig. 4.

\section{III.2.2 Strength Calculation}

The separate four-turn-windings are inter-connected to produce periodic and systematic perturbations along the 7 last achromats in a way which we will describe below. Each circuit is presently powered with bi-polar HCOR12 supplies limited to \pm 5 amperes by the voltage reguirement. With four turns in each circuit and with the main Arc magnets powered with about 4000 amperes, the maximum strength perturbation of each magnet is of the order of \pm 0.005 .

Hore precisely, the magnitudes of the nominal and incrementa! dipole and quadrupole components ${ }^{\dagger}$ which are generated on the central trajectory have been calculated with POISSON, for Arc-type magnet, norninally powered with 3766 amperes in the main coil, and trimmed with 20 ampere-turns in the backleg windings ${ }^{5}$.

When the top and bottom windings are perturbed with the same polarity. mid-plane symmetry is preserved and the strengths of the horizontally deflecting dipole field (vertical magnetic field), and of the regular quadrupole component in the combined function magnets are perturbed.

When the top and bottom windings are perturbed with opposite polarity a vertically deflecting dipole field (horizontal magnetic field), and a skew quadrupole component are generated. The magnitudes of the components are listed below:

1. Nominally powered magnet: horizontal dipole $=5701.60$ Gauss, regular quadrupole $=715.64$ Gauss $/ \mathrm{mm}$.

2. Trimmed magnet with same polarity for top and bottorn coils: incremen-

- The bottom windings on each coil have in some magnets been observed to droop and to cause partial shorts. As a preventive measure, the last turn was cut off and disconntected on each of the modified coils.

f We neglect the perturbation to the sextupole component, which is very small (less that 0.005 of the nominal value) and for which the colerance for sizeable effects on the beam is inose (a few percent). 
tal borizontal dipole $=27.87$ Gauss, incremeutal regular quadrupole $=3.49$ Gauss $/ \mathrm{mm}$. The magnitudes of the perturbations are close to equal in the focusing and defocusing magnets, and correspond to a 0.00488 of the nominal values.

3. Trimmed focusing magnet with opposite polarity for top and bottom coils: incremental vertical dipole $=14.05$ Gauss, incremental skew quadrupole $=$ 1.59 Gauss $/ \mathrm{mm}$. This corresponds to 0.0022 of the nominal values.

4. Trimmed defocusing magnet with opposite polarity for top and bottom coils: incremental vertical dipole $=16.58$ Gauss, incremental skew quadrupole $=$ 2.15 Gauss $/ \mathrm{mm}$. This corresponds to 0.0030 of the nominal values.

It can be noted that the skew components have about half the strength of the regular components. In addition, the magnitudes of the effects from trimming the focusing and defocusing magnets with opposite polarity for top and bottom coils are slightly assymetric. This may arise from an assymetry in the pole shape which exists between the two magnets.

\section{III.2.3 Polarities of Components}

We use the TRANSPORT polarity convention ${ }^{11}$. We have determined that:

1. A positive trim current in both top and bottom coils, to generate regular quadrupole components, will strengthen both focusing and defocusing magnets. This means that both the total bending angle and quadrupole component become stronger.

2. A positive trim current in the top coil and a negative trim current in the bottom coil, to generate skew quadrupole components, will generate a negative vertical kick in the defocusing magnets, a positive kick in the focusing magnets, and a regative skew quadrupole in both focusing and defocusing magnets.

\section{III.3 Magnet Interconnections - Wiring and Layout}

\section{III.3. I Intermagnet Wiring}

Each trim winding, top or bottom, is connected in series with equivalent windings, top or bottom, exactly five cells (corresponding to $3 \pi$ betatron phase-advance) (or ten magnets) apart, along the 7 last achromats (70 cells or 140 magnets) in the 
Arcs. Since focusing and defocusing magnets are wired separately, there are thus 20 independent circuits. The wiring arrangement is illustrated in Fig. 5.

III.3.2 Nomenclature

The database formal names for the 20 supplies are listed below.

SMLS, CA13,1703=Top coii of defocusing magnet.

SMPS,CA13,1704=Bottom coil of defocusing magnet.

SMPS,CA13,1708=Top coil of focusing magnet.

SMPS,CA13,1709=Bottom coil of focusing magnet.

SMPS,CA13,1713=Top coil of defocusing magnet.

SMIPS,CA13,1714=Bottom coil of defocusing magnet.

SMPS,CA 13,1718=Top coil of focusing magnet.

SMPS,CA13,1719=Bottom coil of focusing tragnet.

SMPS,CA13,1723=Top coil of defocusing magnet.

SMPS,CA13,1724=Bottom coil of defocusing magnet.

SWPS,CA13,1728=Top coil of focusing magnet.

SMIPS,CA13,1729=Bottom coil of focusing magnet.

S.TPS, CA13,1733=Top coil of defocusing magnet.

SMPS, $\mathrm{CA13}, 1734=$ Botton coil of defocusing magnet.

SMPS, CA13,1738=Top coil of focusing magnet.

SMIPS,CA13,1739=Bottom coil of focusing magnet.

S.MPS, CA 13,1743=Top coil of defocusing magnet.

SMPS,CA 13,1744=Bottom coil of defocusing magnet.

SMPS,CA13,1748=Top coil of focusing magnet.

S.IPS,CA13,1749=Battom coil of focusing magnet.

The last digit of the unit number $(3,4,8,9)$ refers to top or bottom windings and to focusing and defocusing magnets. The next to last digit $(0,1,2,3,4)$ refers 
to the cell number of the first coil in the each string. The first two digits (1T) refer to the achromat number of the first coil on each string.

\section{III.3.3 Multiknob Definitions}

The nine harmonic perturbations defined in III.1 are produced by linearly combining the 20 above supplies according to the coefficients given in the same paragraph. using the software multiknob facility ${ }^{18}$. The table below gives the mapping relating the 20 supplies to the 9 knobs:

\begin{tabular}{|c|c|c|c|c|c|c|c|c|c|}
\hline & $\operatorname{sinxx}$ & $\cos x \mathrm{x}$ & SI.NYY & Cosy $\mathrm{Y}$ & SINXY & $\cos \mathrm{XY}$ & SYSEEW & SYSROI & FŨisiB \\
\hline 1703 & +0.00 & +0.00 & -0.59 & -0.81 & -0.59 & -0.81 & +1.00 & +1.00 & $\div 1.00$ \\
\hline 1704 & +0.00 & +0.00 & -0.59 & -0.81 & +0.59 & +0.81 & -1.00 & -1.00 & +1.00 \\
\hline lios & -0.59 & -0.81 & +0.00 & +0.00 & -0.79 & -1.09 & +1.35 & -1.35 & -1.00 \\
\hline 1709 & -0.59 & -0.81 & +0.00 & +0.00 & +0.79 & +1.09 & -1.35 & +1.35 & -1.00 \\
\hline 1713 & +0.00 & +0.00 & +0.95 & +0.31 & +0.95 & +0.31 & +1.00 & +1.00 & +1.00 \\
\hline $171 t$ & +0.00 & +0.00 & +0.95 & +0.31 & -0.95 & -0.31 & -1.00 & -1.00 & +1.00 \\
\hline 1718 & +0.95 & +0.31 & +0.00 & +0.00 & $+1.2 \mathrm{~s}$ & +0.42 & +1.35 & .1 .35 & -1.00 \\
\hline 1719 & +0.95 & +0.31 & +0.00 & +0.00 & -1.28 & -0.42 & -1.35 & +1.35 & -1.00 \\
\hline 1723 & $\div 0.00$ & +0.00 & .0 .95 & +0.31 & -0.95 & +0.31 & +1.00 & +1.00 & +1.00 \\
\hline 1724 & +0.00 & +0.00 & .0 .95 & +0.31 & +0.95 & -0.31 & -1.00 & -1.00 & +1.00 \\
\hline 1725 & -0.95 & +0.31 & +0.00 & +0.00 & $-1.2 \mathrm{~s}$ & +0.42 & +1.35 & -1.35 & -1.00 \\
\hline 1720 & .0 .95 & +0.31 & +0.00 & +0.00 & $+1.2 \mathrm{~S}$ & -0.42 & -1.35 & +1.35 & -1.00 \\
\hline 1733 & +0.00 & +0.00 & +0.59 & -0.81 & +0.59 & -0.81 & +1.00 & +1.00 & +1.00 \\
\hline 1734 & +0.00 & +0.00 & +0.59 & -0.81 & -0.59 & +0.81 & -1.00 & -1.00 & +1.00 \\
\hline 1.35 & +0.59 & -0.81 & +0.00 & +0.00 & +0.79 & -1.09 & +1.35 & -1.35 & -1.00 \\
\hline 1,30 & +0.59 & -0.81 & +0.00 & +0.00 & -0.79 & +1.09 & -1.35 & +1.35 & -1.00 \\
\hline $17+3$ & +0.00 & +0.00 & +0.00 & +1.00 & +0.00 & +1.00 & +1.00 & +1.00 & +1.00 \\
\hline 174 & +0.00 & +0.00 & +0.00 & +1.00 & +0.00 & -1.00 & -1.00 & -1.00 & +1.00 \\
\hline $17+5$ & +0.00 & +1.00 & +0.00 & +0.00 & +0.00 & +1.35 & +1.35 & -1.35 & .1 .00 \\
\hline $1 \div+9$ & $\div 0.00$ & +1.00 & +0.00 & +0.00 & +0.00 & -1.35 & -1.35 & +1.35 & -1.00 \\
\hline
\end{tabular}


Only seven of these nine knobs bave been connected and used. The two missing ones are the second harmonic skew modulations: SINXY and COSXY. It can be noted that for the skew multiknobs, the focusing magnets have coefficients which are scaled by a factor of 1.35 with respect to those of the defocusing magnets, in order to account for the assymetry between the skew components which was noted in III. 2.2 


\section{SIMULATION OF EFFECTS}

\section{V.1 Introduction to Simulation}

We have used ARCSIMI1 ${ }^{19}$ and TRANSPORT ${ }^{11}$ to simulate the effects from focusing perturbations at zero and twice the betatron frequency.

ARCSIMI is a fast simulation which treats small perturbations of the Arc magnets through a linear expansion around the design optical transfer matrix. The overall perturbed optical transfer is then reconstructed by multiplying each individually perturbed Are matrix.

ARCSINI is ideally suited to study the physics of the harmonic focusing perturbations. However, in its current version, ARCSAMl does not include steering effects from the combined function magnets. Such steering effects do not modify the physics but do modify the magnitude of the effects. In effect, through the sexcupoie component in the combined function magnets, horizontal deflections will cause regular quadrupole perturbations and vertical deflections will cause skew quadrupole perturbations. Because the strength perturbations are at zero or twice the betatron frequency, the trajectory excursions from these deflections, and the induced optical effects, will also be at zero or twice the betation frequency. The optical effects will thus add to or subtract from the optical effect from the perturbations of the quadrupole components themselves. In addition, the perturbations will also cause net trajectory deviations" at the end. As will be seen, the trajectorv deviations are large enlough to require a correction. To correctly estimate the magnitude of the combined effects, we have used a pert,urbed TRANSPORT deck of the Arcs, in second order and including steering effects.

We first show the maximum effects of the first order optical distortions from the nine multiknobs defined above, in a perfect planar SLC Arc lattice. We do rot consider second order optica! distortions which arise from deviations in the achromaticity of the optical transfer caused by the first order distortions. Such effects have not been calculated in detail, but are estimated to be small.

Effects from rolls, which are present in the stretch of Arc lattice where the harmonic correction is introduced, will result in not fully orthogonal controls. The

\footnotetext{
- In theory. trajectory deviations would be resonant if the deffections were at the betatron frequency, and not at zero or twice the betatron frequency In res.lity, because of the phaseslippage induced by the second harmonic (see II.6.1), the steering effects will grow slightly as the cancellations of deffections $\pi$ apart no longer occus perfectly.
} 
magnitude of the effects is however not strongly affected, since the component of the perturbed phase-space which is coupled into the other plane across each rol! is not driven in that plane. In addition the component of the perturbed phase-space which is coupled into the other plane is not very large. To illustrate this point, we show the magnitude of one of the optical distortions in a lattice with the actual rolls installed in the present North Arc.

The plots shown are made with ARCSIM1, but the strengths of the knobs have been adjusted so that the maximum effect at the end of the seven achromat long stretch corresponds to the magnitcie calculated with TRANSPORT, inciuding the steering effects (this fudging exercise nas however not been done for the phasing of the knobs).

We then show one simulated example of empirically correcting a lattice j-erturbed by errors with the harmonic corsectors defined above. In the case of a systematic regular focusing error, we also illustrat the bandwidth limit discussed in II. 10.

\section{IV.2 Simulated Effects of Harmonic Correctors}

\section{IV.2.1 One-Dimensional Oscillations}

Case $\nu \cong 0: F D$-Iribalance

A nominal horizontal oscillation is shown in Fig. 6a. The same oscillation, but with the focusing and defocusing magnets perturbed through systematic regular focusing errors of \pm 0.005 respectively is shown in Fig. 6b. As can be seen the amplitude of this oscillation is perturbed negligibly, but its frequency is stifted. It can be calculated that the corresponding shift, includiug optical effects from the displacement of the trajectory, is ${ }^{9}$ :

$$
\left(\Delta \mu_{x}, \Delta \mu_{y}\right) \simeq( \pm 87 \mathrm{deg} . \mp 115 \mathrm{deg}) .
$$

The trajectory displacement can also be calculated: it is $42 \mu \mathrm{m}^{9}$. Both agree well with simulated values.

A plot of the unperturbed and porturbed horizontal beam envelopes is shown in Fig. 7a,b. As can be seen, the keam envelopes are negligibly perturbed.

Case $\nu \simeq 2$ : Regular Second Harmonic

Two one-dimensional horizontal oscillations with the same initial amplitude, but with two different initial phases separated by $90 \mathrm{deg}$, and with the focusing 
magnets perturbed through regular focusing periodic modulation at twice the betatron frequency, with amplitude \pm 0.005 , are shown in Fig. 8a,b. The two initial phases have been chosen to obtain a maximally growing and a maximally decaying oscillation. As can be seen, the predicted maximum growth is about a factor four. The beats generated in the horizontal and vertical beam envelopes are shown in Fig. 9a,b. As can be seen, the orthogonality of the focusing and defocusing magnets which was noted in 11.4 .1 is almost perfect (i.e. there is almost no effect on the vertical beam envelope). This orthogonality is however not fully preserved if rolls are present in the lattice. To illustrate this point, we show in $9 c, d$ the beats produced in the horizontal and vertical beam envelopes, from the same perturbation as in $9 a, b$ applied to the North Arc with the present roll configuration. A reduction of the effect in the horizontal plane and some coupling into the vertical plane can be seen".

Identical effects can be obtained for vertical oscillations with the defocusing magnets perturbed through regular focusing periodic modulation at the twice ine betatron frequency.

The displacement of the trajectory is nearly $300 \mu \mathrm{m}$ from these knobs. This is large and requires a correction at the very end in order to be able to launch into the Final Focus.

\section{IV.2.2 Two-Dimensional Oscillations}

\section{Case $\nu \simeq 0:$ Systematic Skew Component}

The coupling of an initially fully horizontal oscillation into the vertical plane, from the systematic skew knob set to its maximum of 5 amperes, is shown in Fig. $10 a, b$. As can be seen the maximum effect is just under $50 \%$, which is rather weak. The same coupling would be obtained for an initially fully vertical oscillation into the horizontal plane, and for any input phase of the oscillation.

Plots of the corresponding beam envelopes are shown ${ }^{\dagger}$, for a nominal initial phase-space in the horizontal plane and for zero initial phase-space in the vertical plane (Fig. 11a,b), and for a nominal equal initial phase-space in both planes (Fig. 12a,b). These two cases correspond to the beating effect described in II.7.2 through (26) and (27) respectively. In the case of nominal initial phase-space in

* A slightly smaller effect from these rollg would have perhaps been seen if the harmonic correction had been installed in a region where the major rolls are matehed. Such a region could have been the region between the beginning of achromat 14 and the end of achromat 20.

$\ddagger$ In order to make the effects more visible, we have calculated the perturbations of the envelopes for a systematic skew perturbation with three times the maxirnum knob strengeh. 
both planes (Fig. 12a,b), the envelopes are not perturbed, as was found in (27).

The displacement of the trajectory from setting this knob at its maximum of 5 amperes is about $30 \mu \mathrm{m}$. This is a small effect (partly because the knob is weak).

Effects from the systematic coordinate rotation are very small and are not illustrated here.

\section{Case $\nu \cong 2$ : Skew Second Harmonic}

The coupling of an initially fully horizontal oscillation into the vertical plane resulting from the skew second harmonic knob set to its maximum of 5 amperes is shown in Fig. 13a,b". As can be seen the maximum effect is quite small. The same coupling would be obtained for an initially fully vertical oscillation into the horizontal plane, and for any input phase of the oscillation.

Plots of the corresponding beam envelopes are shown ${ }^{\dagger}$, for a nominal initial phase-space in the horizontal plane and zero initial phase-space in the vertical plane (Fig. 14a,b), and for a nominal initial phase-space in both planes (Fig. 15a,b). These two cases correspond to the exponentially growing cross-plane coupling effects which were described in II.T.2 through (30) and (3l) respectively.

The displacement of the trajectory from setting this knob has not been calculated in detail but appears to be large enough to require a correction at the very end. to launch properly into the Final Focus System.

\section{TV.3 Systems with Errors}

\section{IV.3.1 Bandwidth Limit from Systematic Error}

In Fig. 9.a, we showed the beat in the one-dimensional horizontal beam envelope caused by a regular second harmonic focusing perturbation. In Fig. 16, we show the same envelope, but now perturbed also by a systematic focusing perturbation cortesponding to $3^{0}$ per cell, or about twice the maximum systematic perturbation which can be caused by the FD-Imbalance. This corresponds to just over twice the maximum cell phase-shift which can be alloved for the harmonic knobs to work properly, as stated in II.10 by (33). As can be seen, the growth

* The two skew second harmonic knobs have not been tried experimentally.

$\dagger$ In order to make the effects mare visible, we have calculated the perturbations of the envelopes for a skew second harmonic perturbation with five times the maximum knob strength. 
in the beating envelope is in this case reversed half way through, and is nearly cancelled at the end.

\section{T.3.2 Empirical Correction of Randomly Perturbed Lattice}

Fig. 17 sbows the horizontal beam envelupe imaged through the seven achromat long stretch, perturbed by random regular focusing errors only, with strengths normally distributed with a standard deviation of 0.01 . The particular "seed" shown in Fig. 17 was chosen as one which generates significant growth of about a factor two. With this magnitude error, such a large growth represents a rather improbable case*. Fig. 18 shows a correction of the case presented in Fig. 17, by combining the sine-like and cosine-like regular focusing second harmonic correctors. Correction is found empirically rather easily for such one-dimensional cases. In lattices fully perturbed with regular and skew focusing errors, the empiriral method is in some cases difficult.

* As pec IV.2.2, a factor two growth can be generaled with a regular focusing modulation at twice the betatron frequeney of amplitude 0.0025 (half the knoo strength). For zandomly distributed regular focusing errors with a standard devjation of 0.01 , the one standard deviation expectation value fot the strength of the component at twice the betatron frequency is $\frac{0.01}{\sqrt{n}}=0.0012$, where $n=70$ is the number of cells in the seven achromats. Thus a 0.0025 stength would cortespond to about two standard deviations of the strength distribution, or about $5 \%$ of the seeds. 


\section{LIMITATIONS AND PROSPECTS}

\section{V.I Strength Limitations}

The strength of the controls provided in the installation described above is severely limited in the case of the cross-plane coupling correctors (the "skew knobs"). Stronger "systematic skew knobs" in particular would be important for future operation with assymetric emittances, to fully cancel the coupling between the two emittances. They do however not appear to be essential for the present operation with close to equal emittances, a case for which they have no or little effect on the beam envelope (see II.7.3).

In addition, in the presence of systematic regular focusing errors of more than one degree per cell, the strength of the correctors for one-dimensional mismatches are significantly weakened. More generally, the strengths of all the correctors are weakened by phase errors distrit ited in the stretch where the harmonic correction is installed.

\section{V.2 Guide-line for Upgrade}

The obvious upgrade would consist in raising the maximum current through the rewired backleg windings, from the present value of about 5 amperes, to twice or three times that. There may be an impediment to doing this from the limited elasticity in the copper wires composing the windings: after the controls have been iurned on. and off a large number of limes, the repeated thermal contractions and dilatations may cause the windings to sag. An enhanced support mechanis $n$ would perhaps be required. The possibility of this sagging is presently being examined.

Another possible upgrade would consist in repeating the insiallation in one or two new stretches upstream of the one described above. Because of the observed tendency of the bottom turn of the backleg windings to droop (see the footnote at the bot tom of page 22), it may be desirable, as a preventive measure, to implement the wiring modification in the remaining 16 achromats. In this case, two new installations similar to the one in achromats $17-23$ could be installed in achromats 9-16 and in achromats 1-7. The existing supplies could in this case be kept, as knobs in adjoining stretches coulc be combined to enhance the effects. The drawback of this is evidently a much increased complexity, as one would then have to deal with 
a theoretical total of 27 knobs, with their relative phasing " and so on".

In summary, if the possible sag in the wires from heating can be solved with a better clamping mechanism, or ntherwise shown not to be a problem, and if $j$ is not essential to implement the wiring modification as a preventive measure for the sagging of the last wire, our recommendation would be to upgrade the power supplies.

\section{V.3 Predictive and Precision Control}

The harmonic corrections are presently performed successfully as approximate and empirical adjustments to control the beam shape at particularly sensitive places at the entrance to the Final Focus, where significant backgrounds can be generated by optical mismatches, or to adjust the overall lattice of the Arcs to be close to nominal'.

More work would be required to design fully predictable model-driven corre:tions for precision control of phase-space parameters at the end of the Arcs. This may be doable by using empirically determined transfer matrices for the Arcs, together with a perturbation technique similar to the one described in IV.1 ${ }^{20}$. Some attempts which were made in this direction have shown that this is not straightforward, and requires a detailed understanding of the propagation of measurement errors involved in the empirical matrix determination. More work in this direction may enable precision controlling the phase-space for future optical optimizations of the SLC Arcs.

- In practice, the coordinate rotations do not matter, so "only" 24 knobs would be used. 


\section{ACKNOWLEDGEMENTS}

Several people have contributed to this work in a major way, and should be acknowledged. Rae Stiening was the first to propose harmonic corrections as a method for adjusting long open FODO arrays. Ted Fieguth pioneered the method in the case of the SLC Arcs, through a system of barmonic steering bumps.

In addition, we have benefited from discussions with Karl Brown, Fatin Bulos, Dave Burke, Dick Early, Jacques Hajssinski, Thomas Lohse, David Ritson, Matt Sands and Nobu Toge.

Finally, we would like to thank the Mechanicai Engineering and Power Supply Groups, and the cable instaliation crews for their support in the installation of "Wirefix". 


\section{REFERENCES}

(1) P. Bambade, "Beam Dynamics in the SLC Final Focus", SLAC-PUB-4227 (June 1987), C. Hawkes and P. Bambade, "First Order Optical Matching in the Final Focus Section of the SLAC Linear Collider", Nucl. Instr. and Meth. A274 (1989) 27 and P. Bambade et al., "Operational Experience with Optical Matching in the SLC Final Focus System", SLAC-PUB-4776 January (1989).

(2) R. Stiening, "Focusing Errors in the SLC Linac Quadrupole Lattice", SLAC-CN-161 (February 1982).

(3) T. Fieguth, "Harmonic Orbit Distortions", Minutes of GIAT Group Meeting (October 1985).

(4) P. Bambade et al., "Initial Operational Experience with Harmonic Corrections in the SLC Arcs", To be published at the Particle Accelerator Conference, in Chicago (March 1989).

(5) D. Early, "Steering Effects in the Main E Bend Magnet", SLAC-CN-216 (January 1983).

(6) P. Bambade, "Betatron Phase-space Diagnostic in a FODO Array", SLACCN-367 (October 1988).

(7) E. Courant and F. Snyder, "Theory of the Alternating Gradient Synchrotron", Anu. of Phys. 3,1-48 (1958).

(8) E. Brigham, "The Fast Fourier Transform", New Jersey, Prentice-Hall, Inc.

(9) J. Haïssinski, Private Communications (April 1987 through January 1989).

(10) A. Nayfeh, "Introduction to Perturbation Techniques", New York, Wiley (1981).

(11) K. Brown et al., "TRANSPORT", SLAC-91, Rev. 2 (May 1977).

(12) E. Wilson. "Circular Accelerators-Transuerse", AIP Conf. Proc. 153, p. $3,(1987)$.

(13) K. Brown and R. Servranckx, "Cross-Plane Coupling and its Effects on Projected Errittances", SLAC-PUB-4679 (July 1988).

(14) L. Teng, "Concerning N-Dimensional Coupled Motions", FermiLab Report F.Y.229 (May 1971). 
(15) The first of the three references in (1).

(16) At this point, this is common knowledge.

(17) P. Bambade et al., "Rollfix - An Adiabatic Roll Transition for the SLC Arcs", SLAC-PUB-4835 (January 1989). (18) Julian Kupiec, "User Guide to the Multiknob Facility".

(19) D. Ritson, ARCSIM, private program (March 1988). A modified version (ARCSIM1) exists with harmonic focusing corrections using regular quadrupoles. Botb versions are installed on the shared SLC 197 disk.

(20) T. Barklow, "A Technique for Measuring the $4 X_{4}$ Transfer Matrix for Beam-Line Sections with Cross-plane Coupling", To be published at the Particle Accelerator Conference, in Chicago (March 1989). 


\section{FIGURE CAPTIONS}

(1) Mismatched one-dimensional phase-space. The beam-size beats between maxima and minima of $M$ and $1 / M$ respectively.

(2) If a systematic regular focusing error is present, the focusing modulatinn being applied and the beat of the beam size become gradually out of phase. This weakens the effect of the harmonic corrector.

(3) Harmonic strength associated to a focusing modulation over a finite length.

(4) Wiring modification of backleg trim windings. Four of the twenty-nine turns are connected to a separate circuit, via the upper right terminal. The twenty-five remaining turns are connected as for the original use of the backleg windings, via the top terminal, except for the bottom, which is removed from the circuit.

(5) There are twenty independent circuits connecting the 280 top and bottom coils of the last seven achromats in the Arcs. In each circuit, top and bottom coils of magnets separated by five cells are connected in series.

(6) Horizontal betatron oscillation in seven achromats of the SLC Arcs, with no errors (a), and with a $1 \%$ FD-Imbalance (b).

(7) Horizontal beam size in seven achromats of the SLC Arcs, with no errors (a), and with a $1 \%$ FD-Imbalance (b).

(6) Horizontal betatron oscillation in seven achromats of the SLC Arcs, with a cosine-like regular quadrupole modulation at twice the betatron frequency in the focusing magnets, corresponding to $\operatorname{COSXX}=5$ Amperes, and for two initial phases amouni'ing to maximum growth (a) and maximum decay (b).

(9) Horizontal and vertical beam sizes in seven achromats of the SLC Arcs, with a cosine-like regular quadrupole modulation at twice the betatron frequency in the focusing magnets, corresponding to $\cos X X=5$ Amperes. Cases (a) and (b) correspond to respectively the horizontal and vertical beam sizes in entirely tat Arcs. As can be seen, the modulation affects mostly the horizontal motion. Cases (c) and (d) correspond to respectively the hurizontal and vertical beam sizes in the last seven achromats of the North Arc, with the present roll distribution. As can be seen, some coupling is generated in the vertical plane, and the beats in the horizontal plane are weakened by about $25 \%$.

(10) Horizontal (a) and vertical (b) betatron oscillation in seven arhromats of the SLC Arcs, with a systematic skew focusing perturbation, corresponding to 
SYSKEW $=5$ Amperes.

(11) Horizontal (a) and vertical (b) beam size in seven achromats of the SLC Arcs, with a systematic skew focusing perturbation, corresponding to SYSKEWV $=15$ Amperes. In these plots, the initial emittance is zero in the vertical plane. An exchange of ascillation energy from the horizontal plane to the vertical plane can be seen.

(12) Horizontal (a) and vertical (b) beam size in seven achromats of the SLC Arcs, with a systematic skew focusing perturbation, corresponding to SYSKEW $=15$ Amperes. In these plots, the initial emittances are equal in the borizontal and vertical planes. In this case, the skew focusing perturbation has no effect on the beam envelopes.

(13) Horizontal (a) and vertical (b) betation oscillation in seven achromats of the SLC Arcs, with a skew focusing modulation at twice the betatron frequency, corresponding to $\operatorname{COSXY}=5$ Amperes.

(14) Horizontal (a) and vertical (b) beam size in seven achromats of the SLC Arcs, with a skew focusing modulation at twice the betatron frequency, corresponding to SYSKEW $=25$ Amperes. In these plots, the initial emittance is zero in the vertical plane. Growth of the phase-space in both the horizontal and vertical planes can be seen.

(15) Horizontal (a) and vertical (b) beam size in seven achromats of the SLC Arcs, with a skew focusing modulation at twice the betatron frequency, corresponding to SYSKEW $=25$ Amperes. In these plots, the initial emittances are equal in the horizontal and vertical planes. Growth of the phase-space in both the horizontal and vertical planes can be seen.

(16) Horizontal beam size in seven achromats of the SLC Arcs, with a cosinelike regular quadrupole modulation at twice the betatron frequency in the focusing magnets, corresponding to $\operatorname{COSXX}=5$ Amperes, and with a systematic focusing perturbation of $1.33 \%$ in the focusing magnets. As can be seen, a beat in the beam size is initiated as in Fig. 9a, but is reversed in the middle of the section and vanishes almost at the end.

(17) Horizontal beam size in seven achromats of the SLC Arcs with the focusing magnets perturbed by random quadrupole errors with a $1 \%$ standard deviation. The particular "seed" was chosen as one which generates large beats at the end.

(18) Horizontal beam size in seven achromats of the SLC Arcs with the focusing magnets perturbed by the same random quadrupole errors as in (17), and corrected 
by superimposing a quadrupole modulation at twice the betation frequency in the focusing magnets, corresponding to $\operatorname{COSXX}=3.9$ Amperes and to SINXX $=0.7$ Amperes. As can be seen, the correction is alniost perfect at the end. 

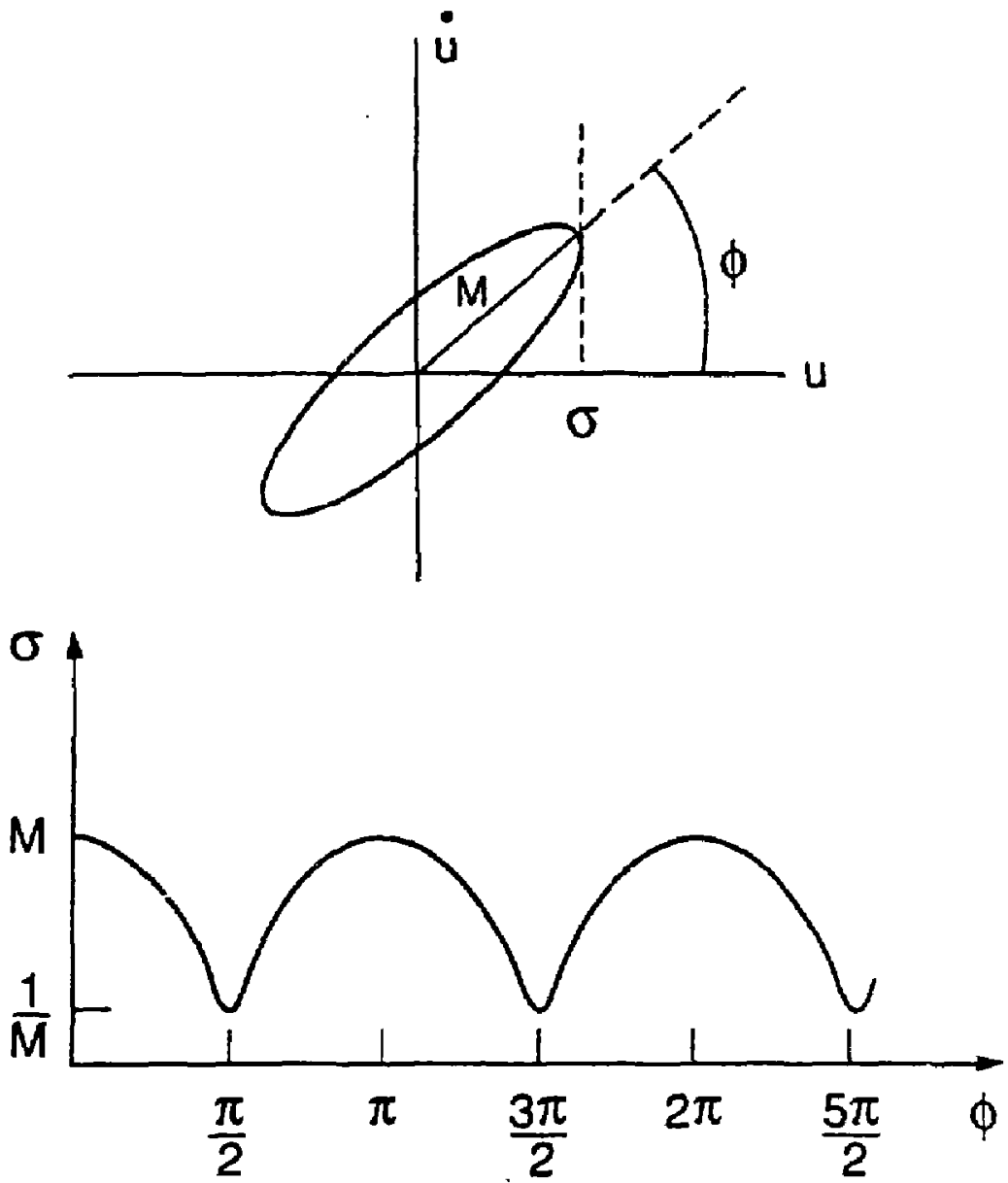

6231A1

Fig. 1 

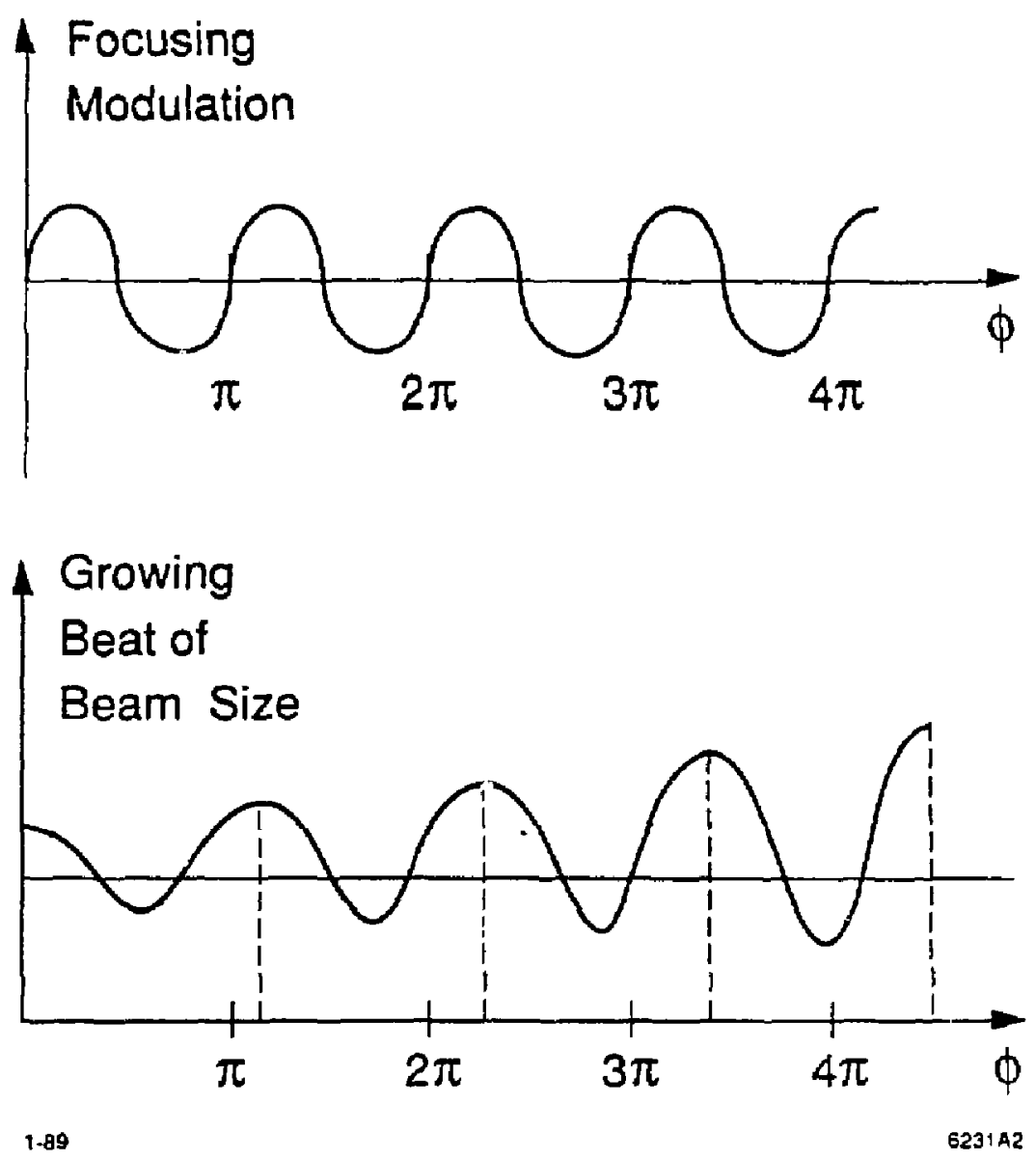

Fig. 2 

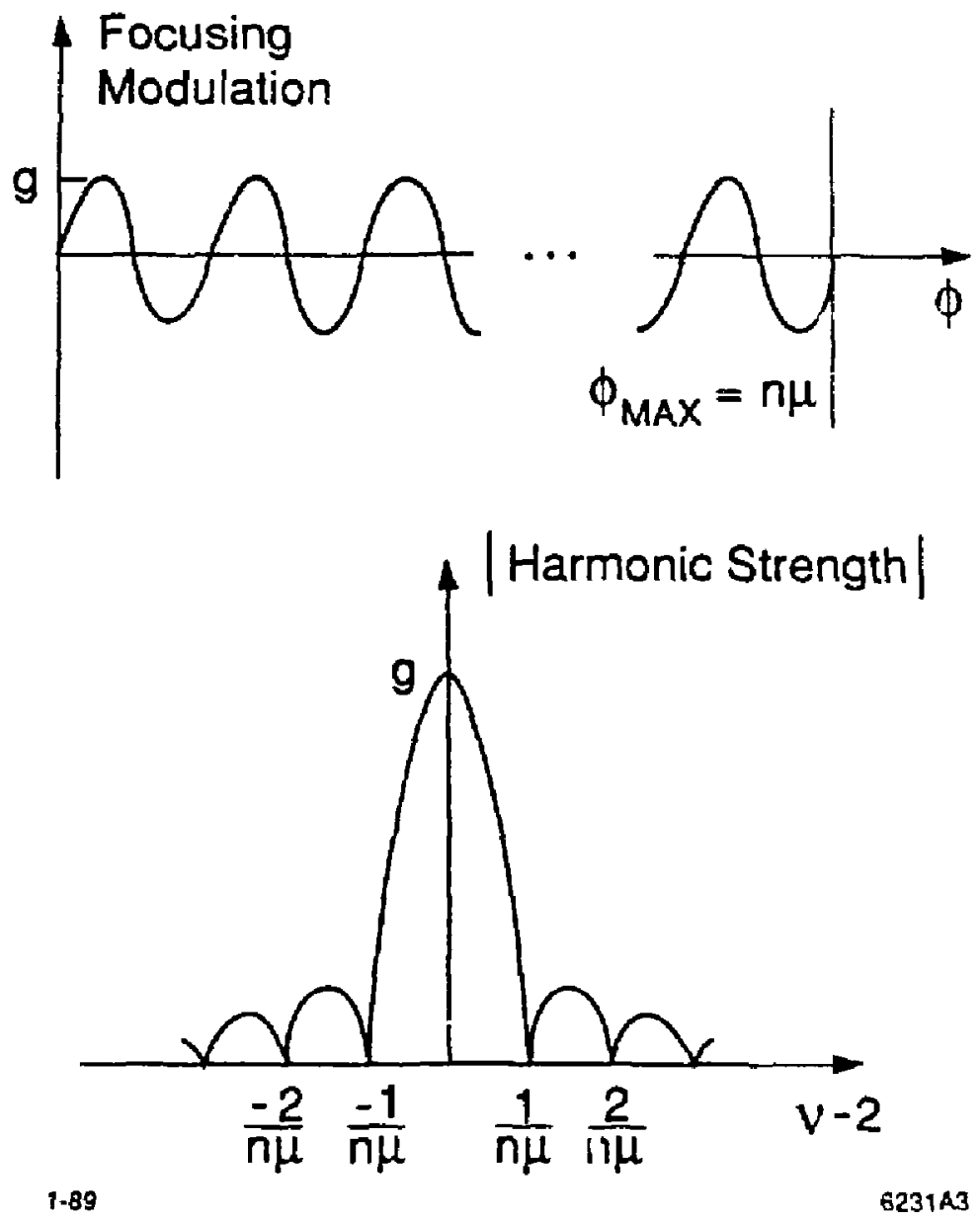

Fig. 3 


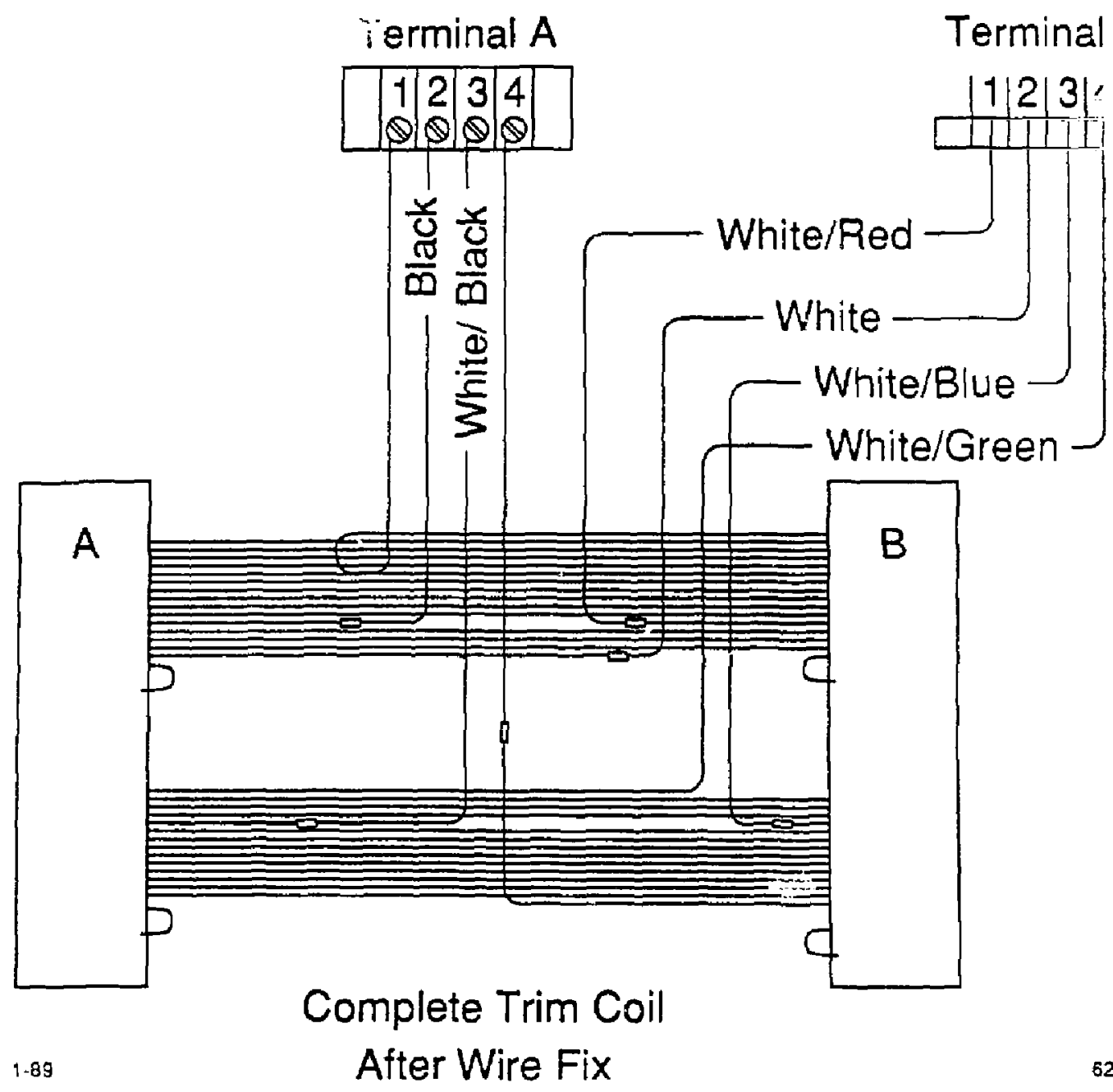

Fig. 4 


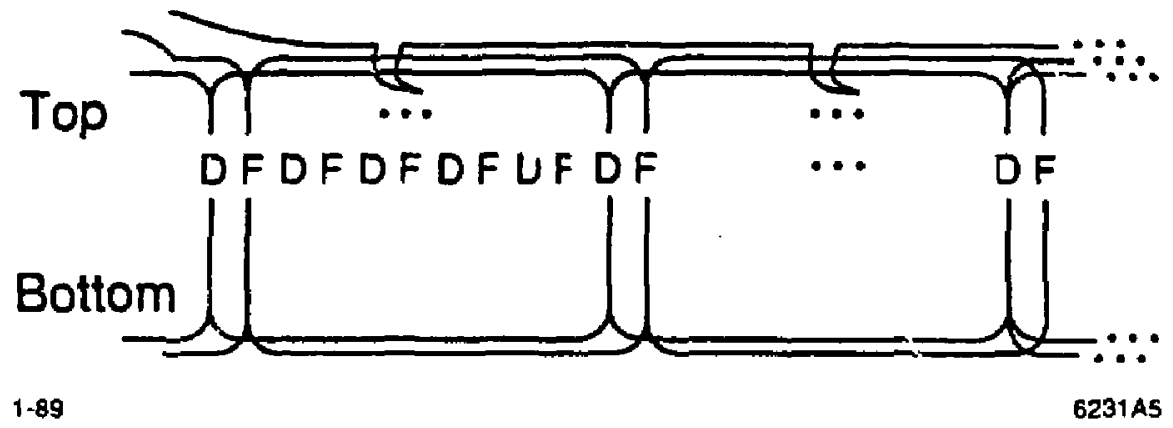

Fig. 5 
$\vec{\Phi}$

$X$ OSCILLATION (mm)

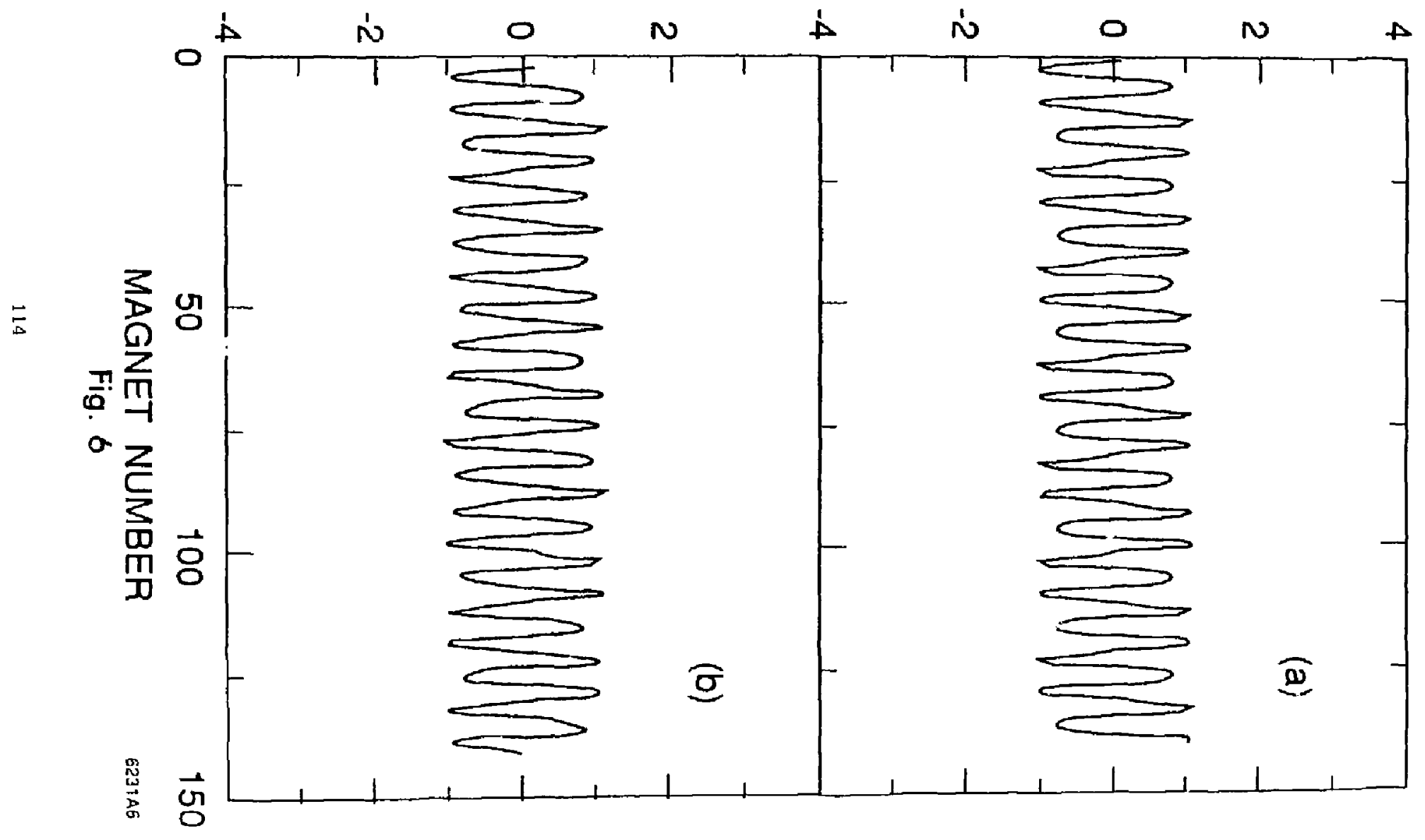




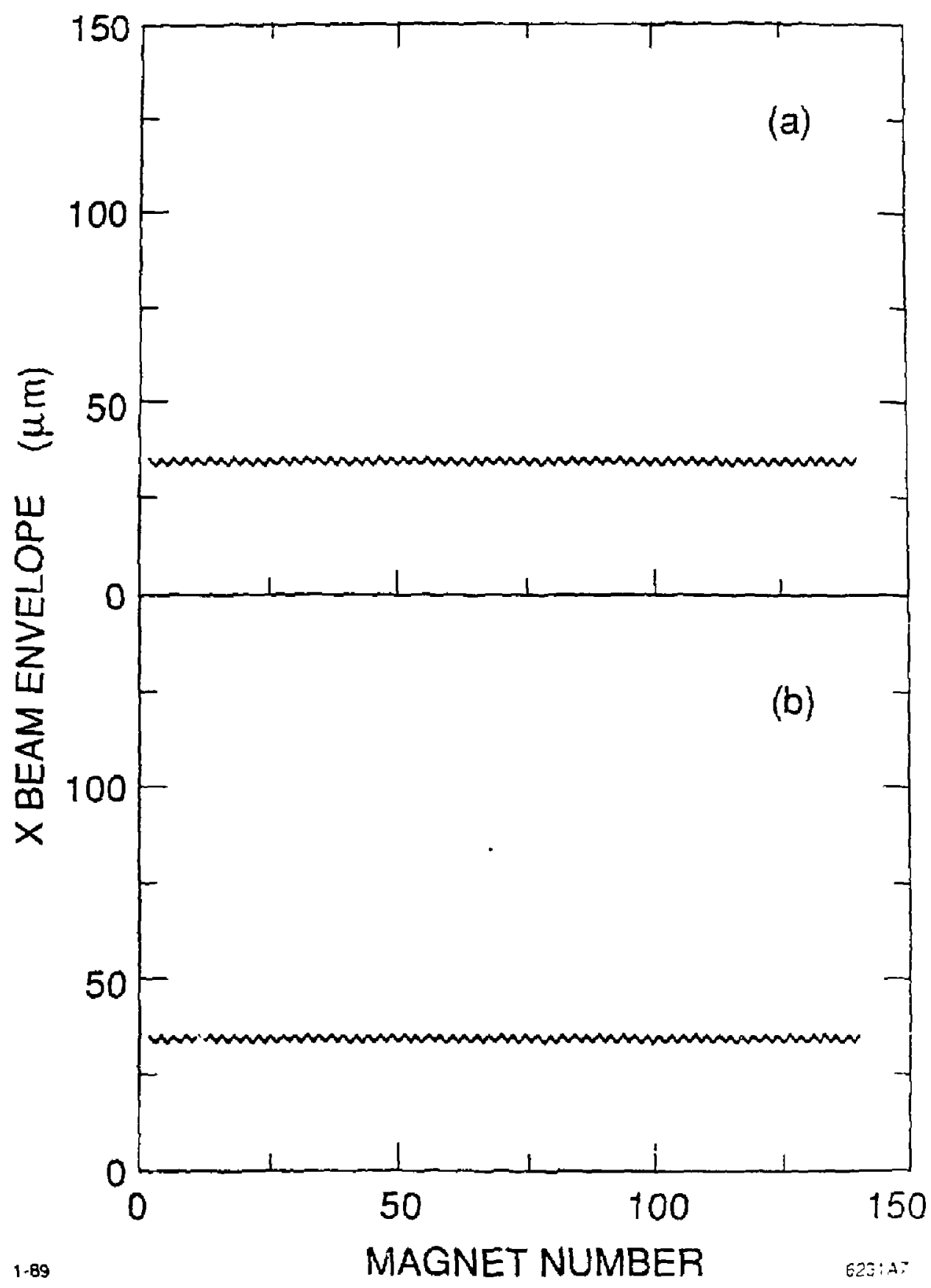

Fig. 7 


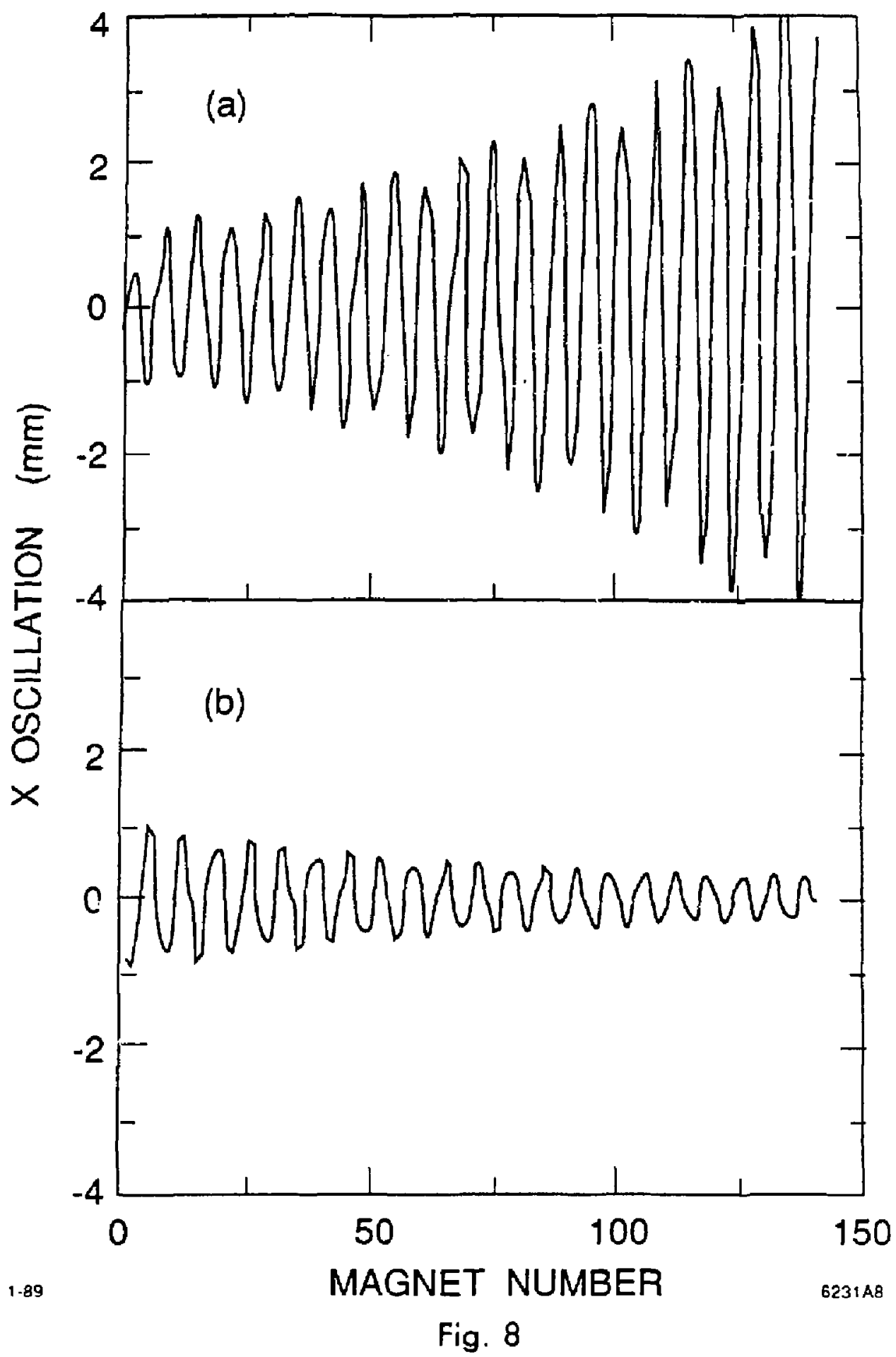




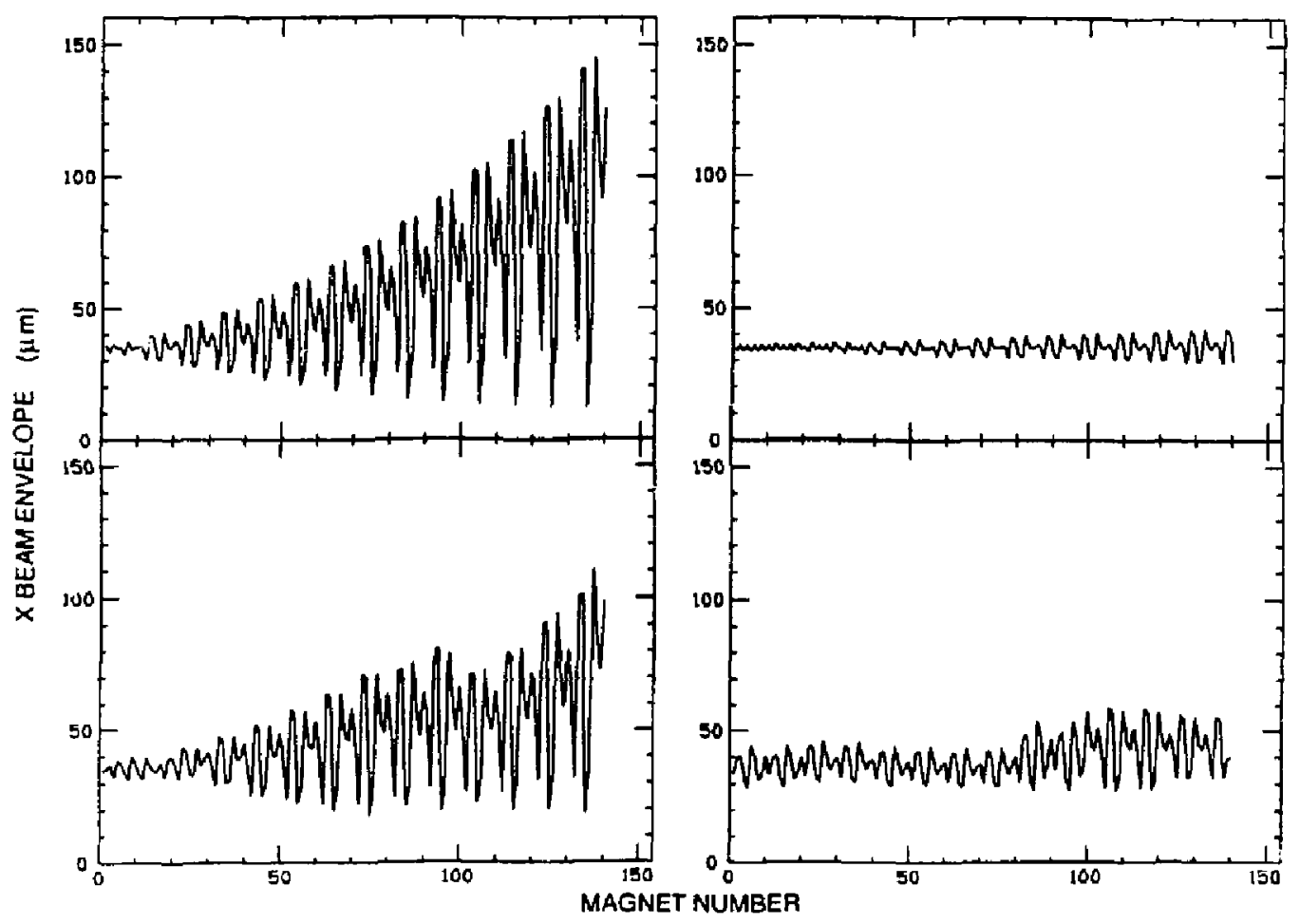

Fig. 9 


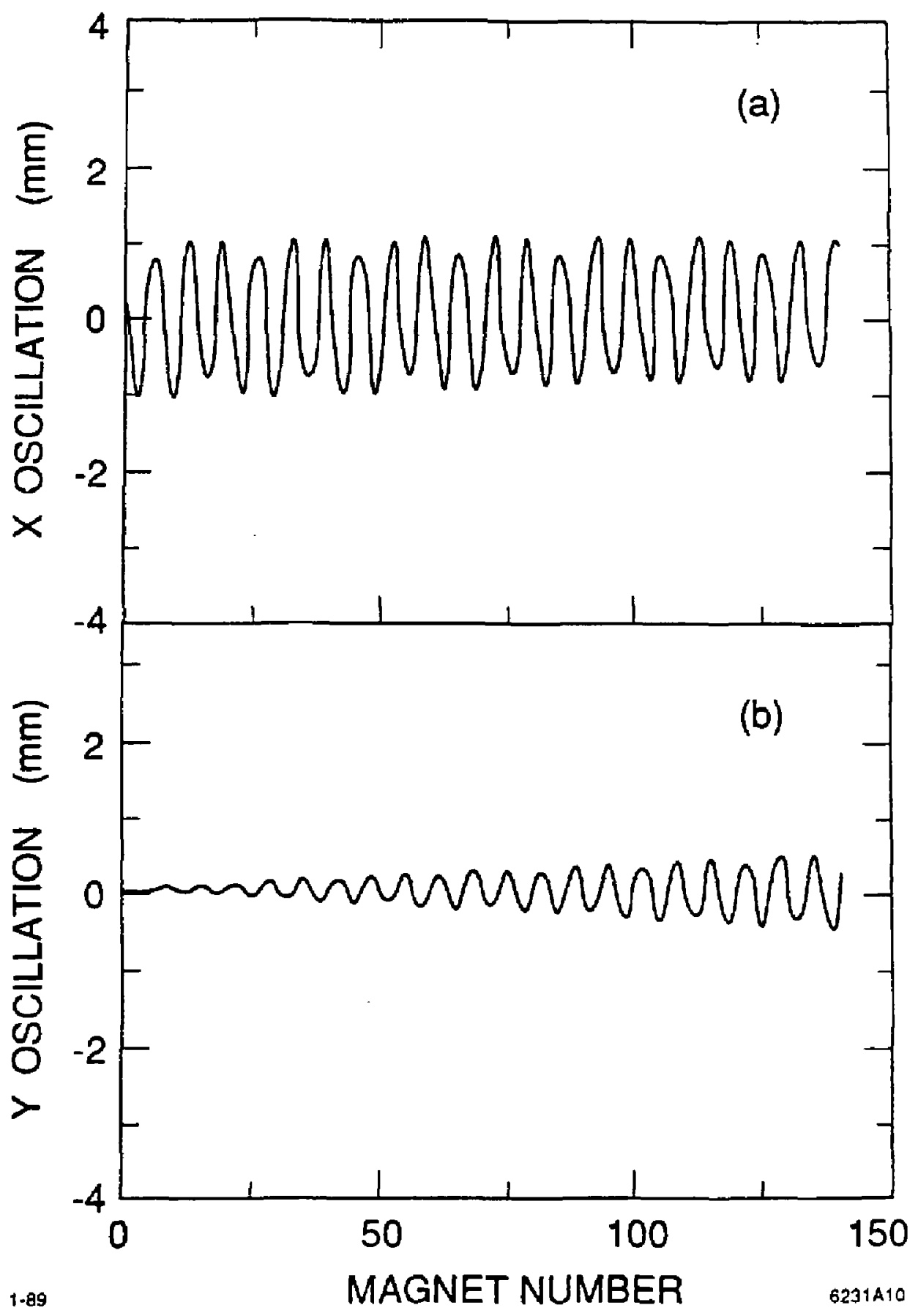

Fig. 10 


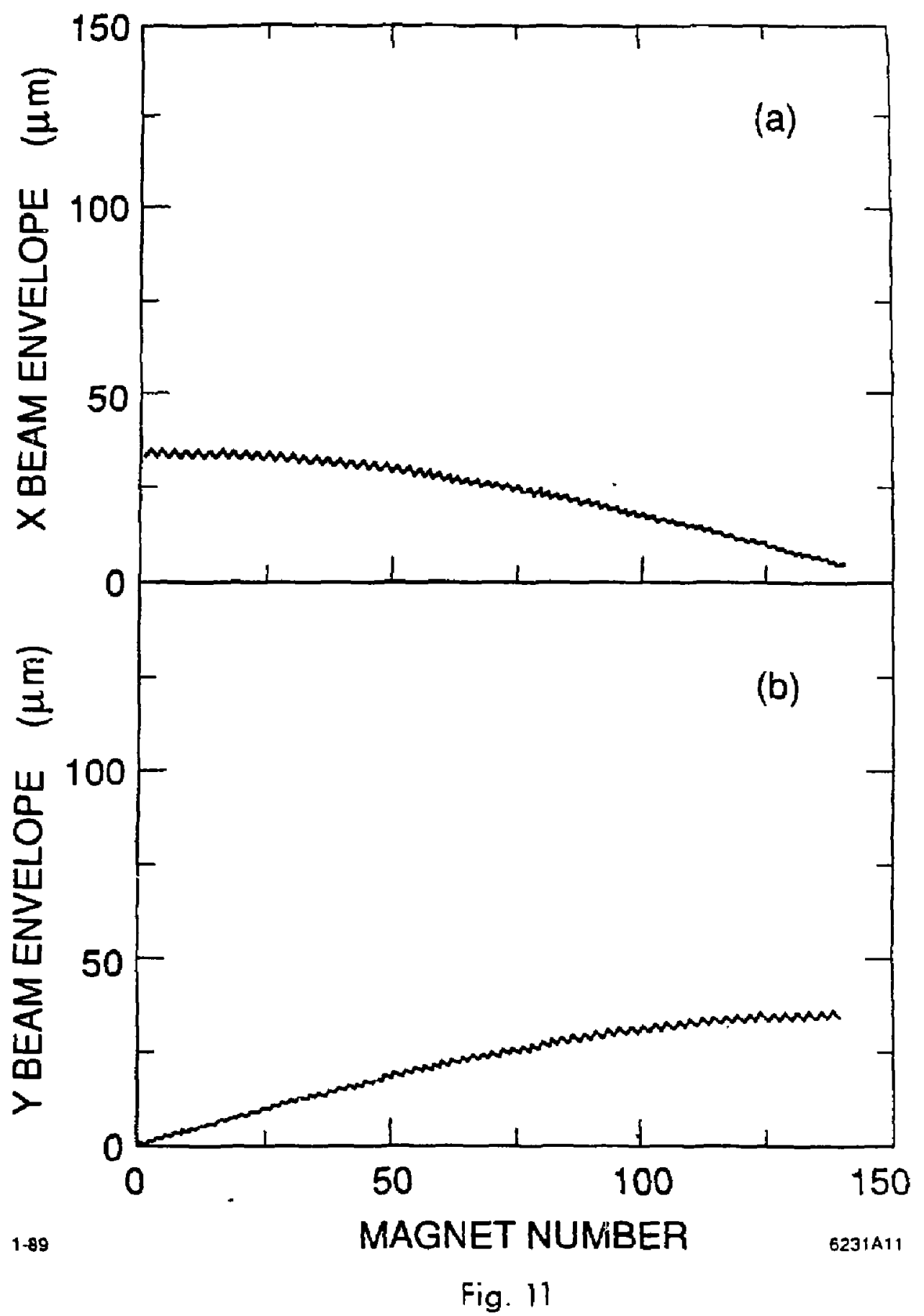




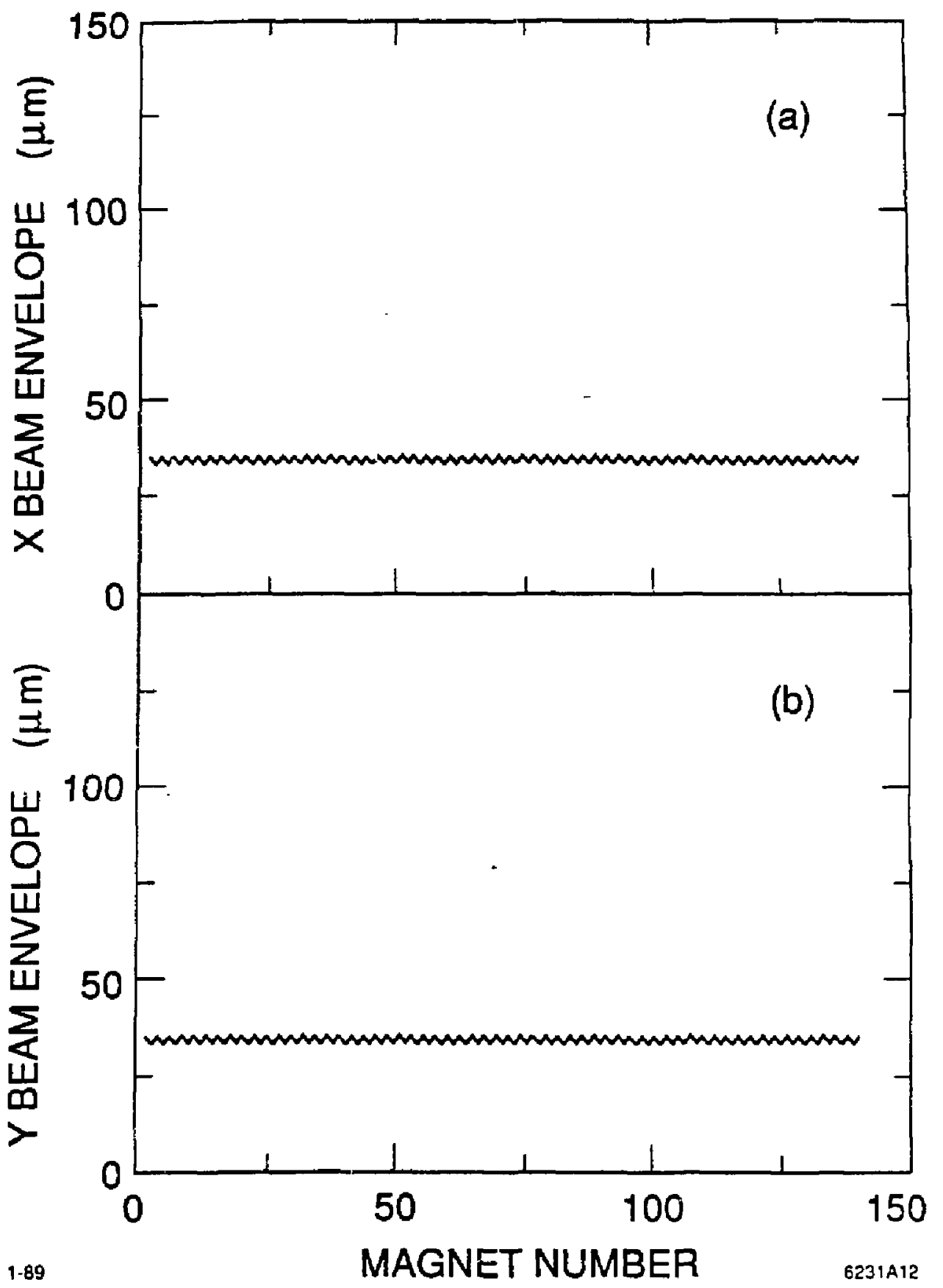

Fig. 12 
$\overrightarrow{8}$

Y OSCILLATION (mm)

$X$ OSCILLATION (mm)

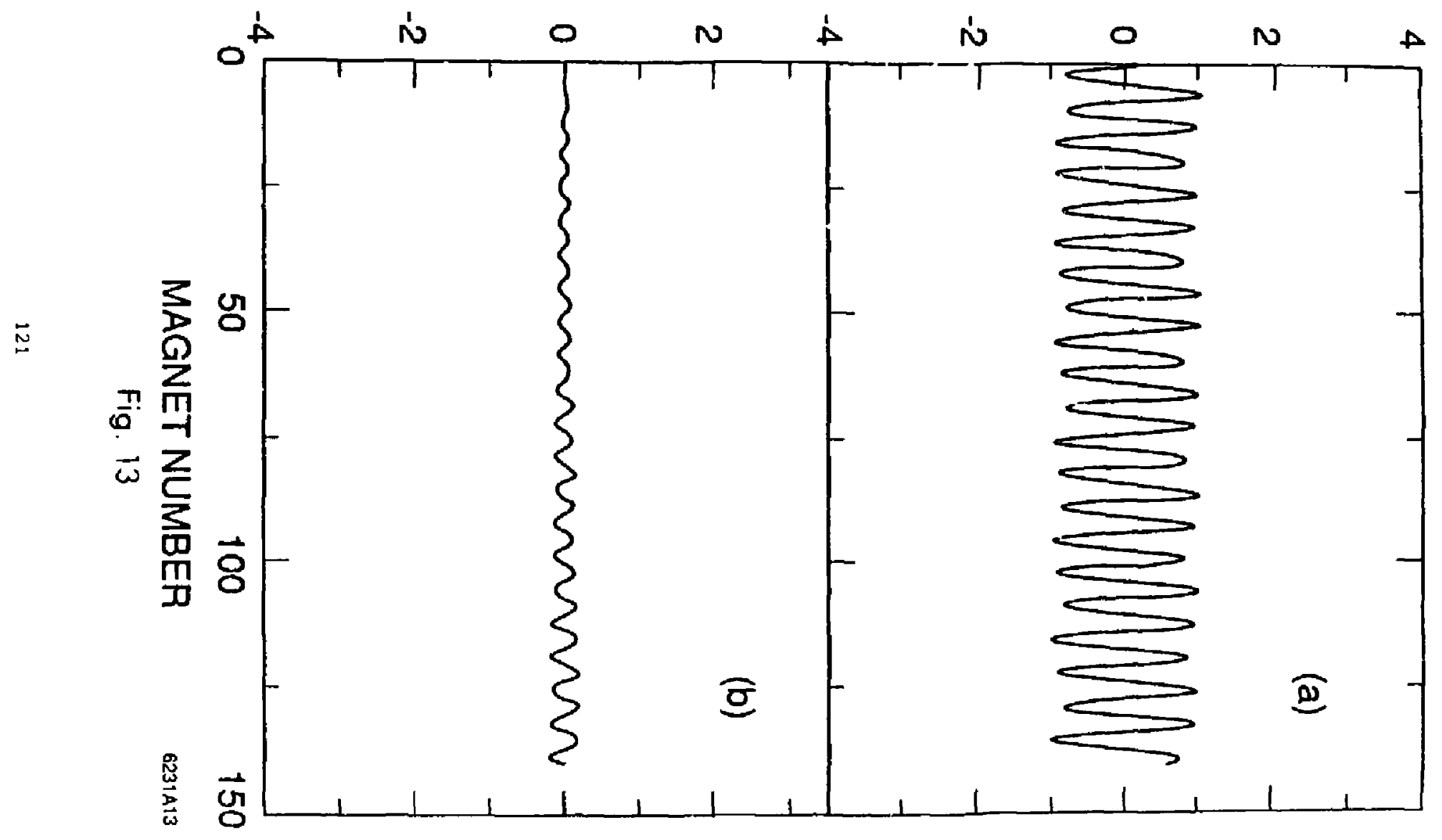




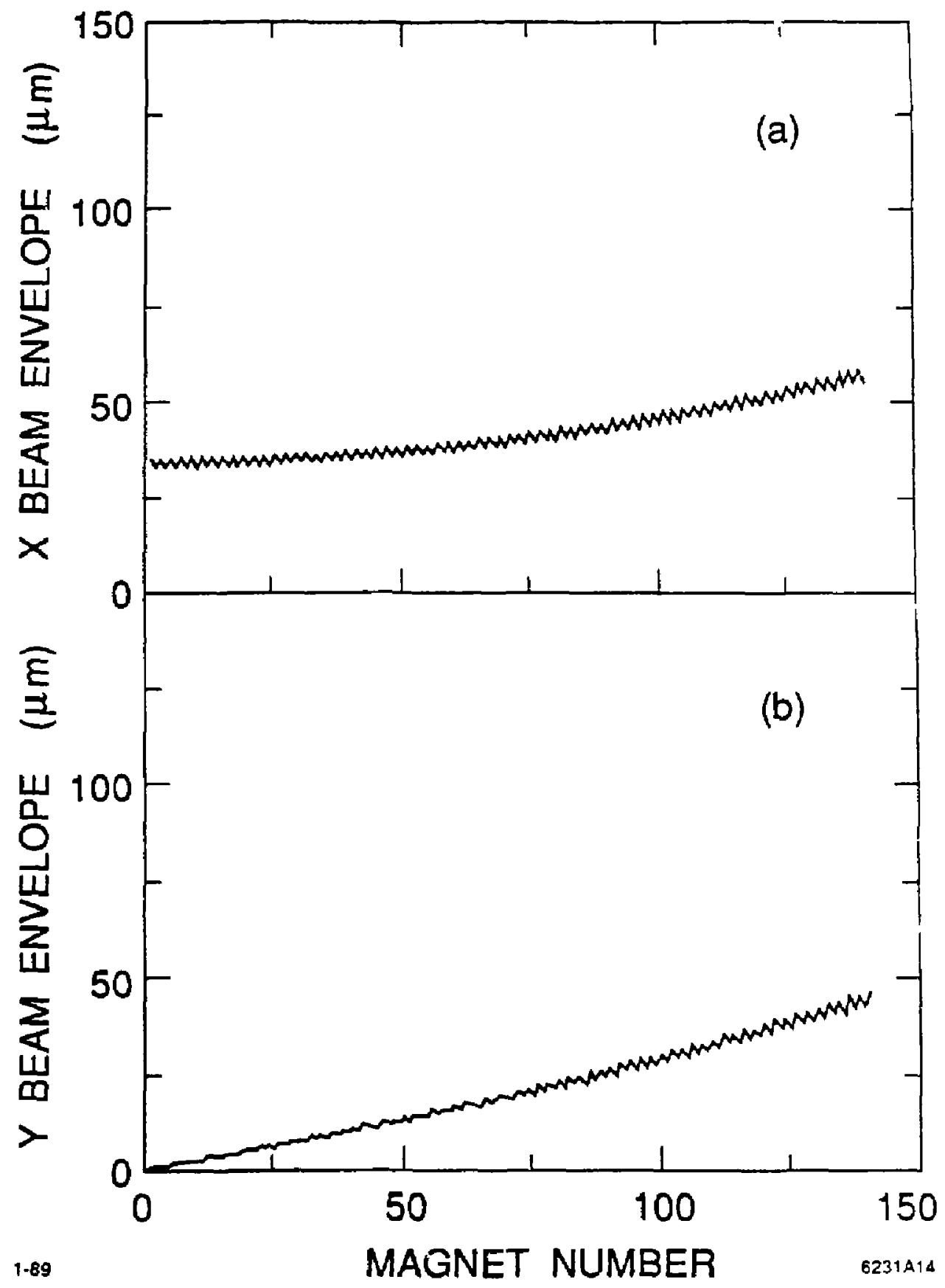

Fig. 14 


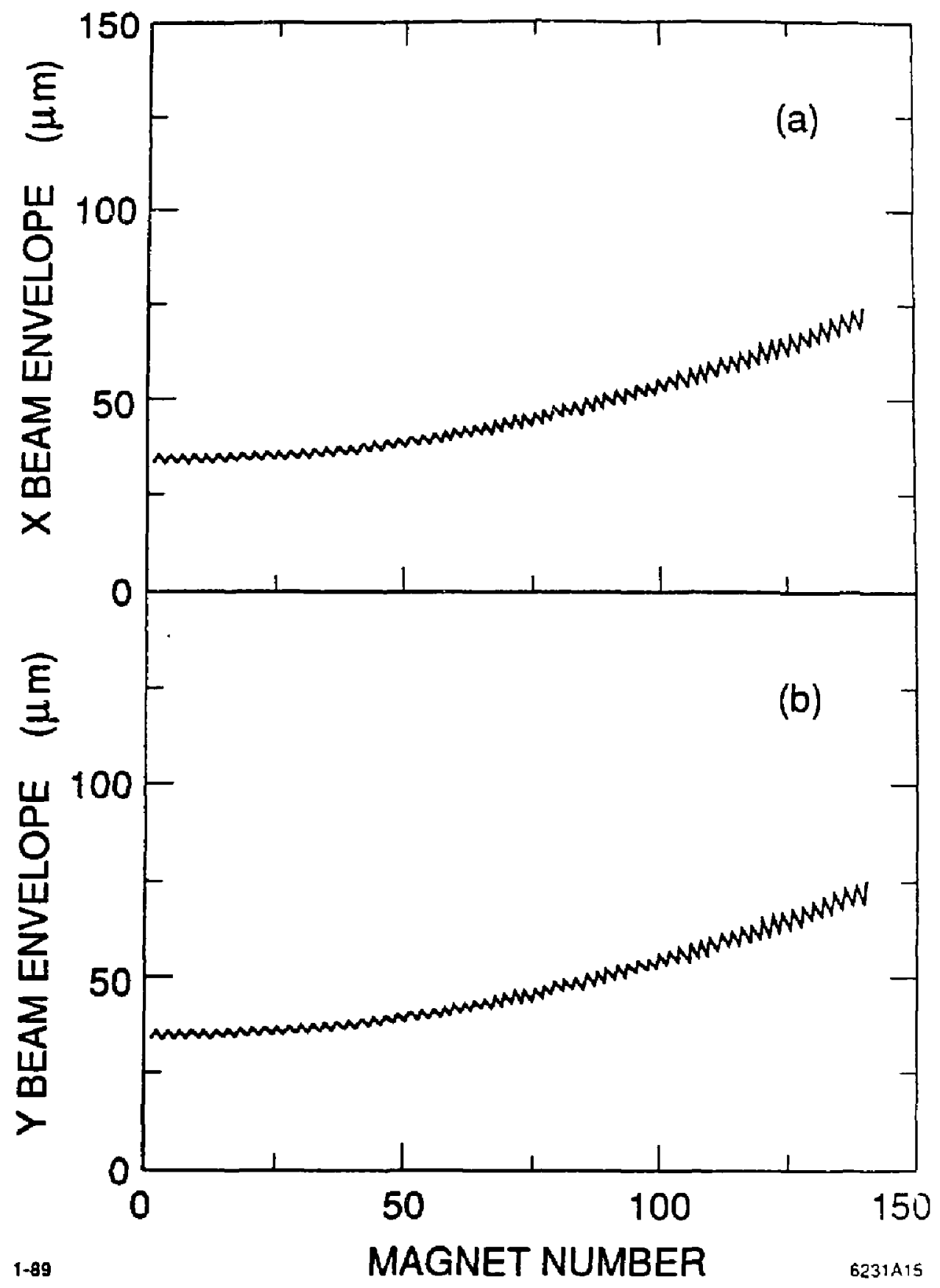

Fig. 15 


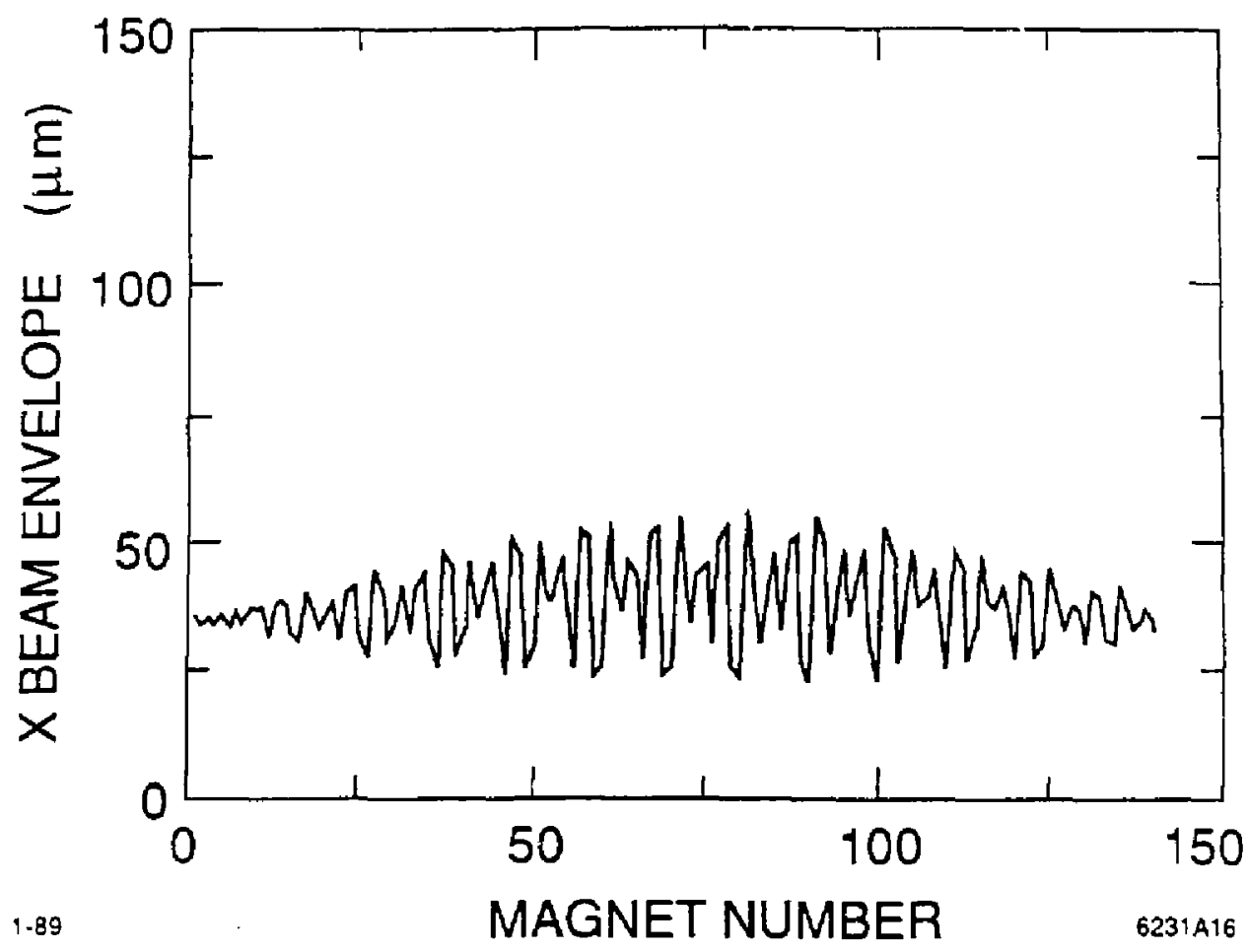

Fig. 16 


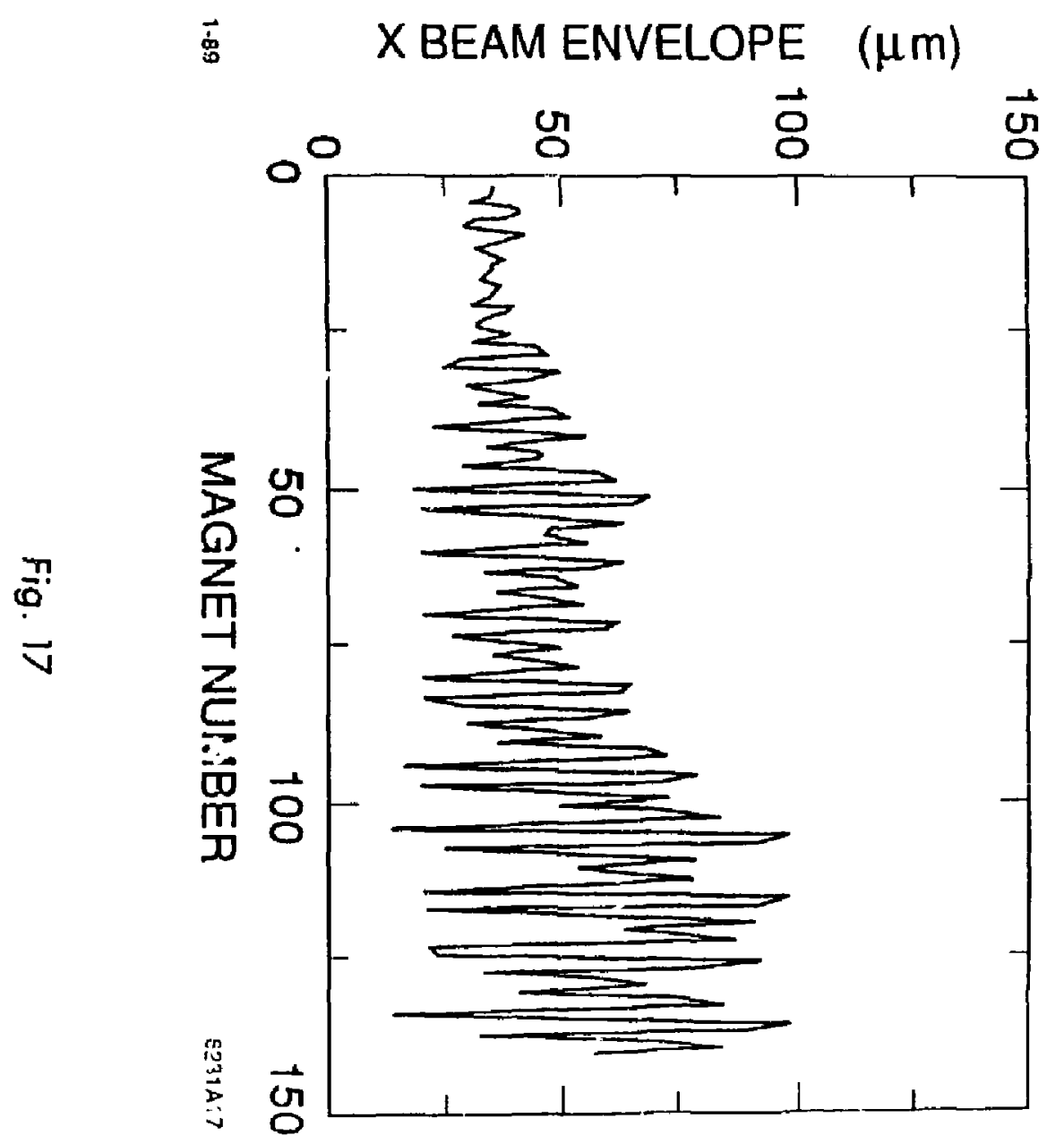




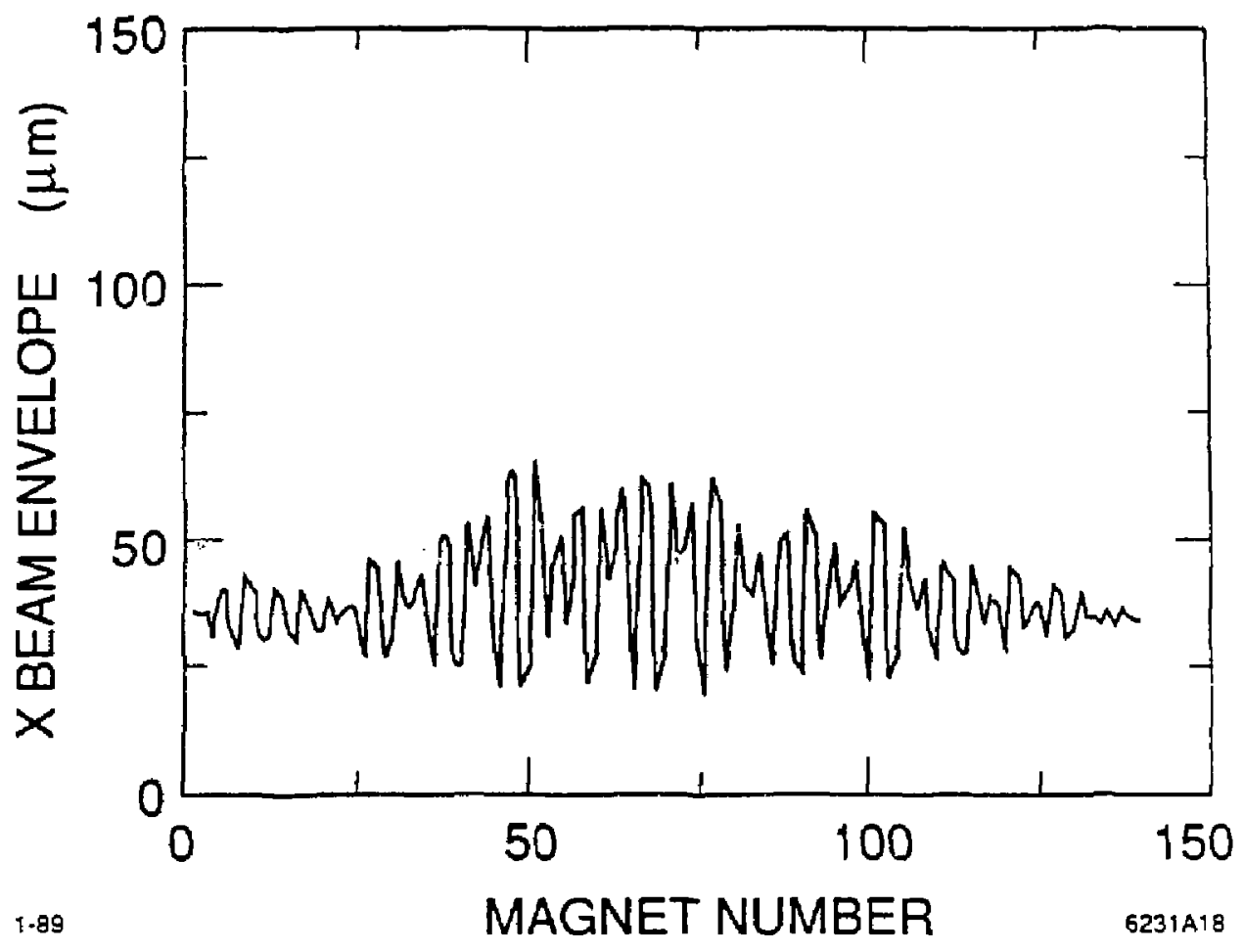

Fig. 18 


\section{VI.4 FIRST TESTS OF HARMONIC CORRECTION METHOD}

In this section, we present the first tests made with the system of harmonic corrections described in VI.3. These tests have shown that it was possible to adjust the phase-space, empirically, at the exit to the arcs, to minimize backgrounds produced by misulatches. Although it has not been possible to study all the cases which were explored in the colluder note above, the measurements we have made confirm qualitatively the theoretical results presented in this note, and the validity of this approach towards adjusting open beam-lines. 


\section{VI.4.1 Resonant Amplification of One-Dimensional Motion}

In Figs. 20a-f and 21a-f, we show a set of betatron oscillations in the north arc and in the first third of the north final focus section*. These oscillations are launched horizontally in the last sector of the linear accelerator. The plots correspond to two different settings of the harmonic correction at twice the betatron frequency, and to two different initial phases for the betatron oscillation. The harmonic correction is applied in the last third of the arc. Figs. 20a-f represent the horizontal transfer of the these oscillations, and Figs. 21a-f the coupling into the vertical plane of these oscillations.

We first concentrate on Figs. 20a-f. Fige. 20a-c and $20 \mathrm{~d}-\mathrm{f}$ correspond to oscillations which are separated by $\pi / 4$ at the origin. In Figs. $20 \mathrm{a}$ and $20 \mathrm{~d}$, no perturbation has been applied. As can be seen, the amplitudes of the oscillations are almost regular in Fig. 20a, while they are slightly damped in Fig. $20 \mathrm{~d}$. In $20 \mathrm{~b}$ and $20 \mathrm{e}$, we have applied, respectively a sine- and a cosine-like regular focusing perturbation at twice the betatron frequency, with an amplitude close to 0.005 of the nominal focusing strength. As can be seen in both cases, the amplitudes of the two oscillations are damped. In $20 \mathrm{c}$ and $20 \mathrm{f}$, we have applied the same perturbations to the same oscillations, but with opposite sign. In this case, the oscillation which was slightly damped (Fig. 20d) becomes regular, and the one which was regular (Fig. 20e) becomes rather strongly amplified.

The effects are in good qualitative agreement with the description given in section II.6.1 of the collider note presented in VL.3: solutions with a maximally perturbed amplitude (damped or amplified) correspond to harmonic perturbations which are $\pi$ out of phase. Furthermore, the effects (damping or amplification) remain unchanged if the phase of the launched betatron oscillation is varied by $\pi / 4$ and if the phase of the harmonic perturbation is varied by $\pi / 2$. This is in agreement with the phase dependance of the effects, as calculated for small perfurbation in section II.5.2 of the collider note mentionred above.

In Figs. 21a-f, we show the coupling into the vertical plane corresponding to the oscillations in Figs. 20a-f. As can be seen, this coupling, already present for the unperturbed oscillations (Figs. 21a,d), is modified rather appreciably by the perturbations we apply. As was explained in section TV.2.1 of the collider note above, this modification results from the rolls introduced in the arcs to follow the terrain of the SLAC site (see section VT.2). This coupling contributes to weaken the perturbation in the horizontal plane. This explains partially the fact that the amplitude of this perturbation is weaker than was predicted, by almost a factor two. The two other reasons for a weaker perturbation than predicted are:

1. The fact that the relative phase of the perturbation and of the betatron oscillation at its origin has not been tuned to better that $\pi / 2$ in these first tests. This relative phase

* In the arcs, the data are row, while in the final focus section, they are scaled with the square roat of the ratio of the $\beta$-functiona in the two respective sections. This enables to make the oscillation comparable in the two section. 
is a priori arbitrary, because of the large distance between the end of the linear accelerator, where the betatron oscillations are launched, and the part of the arc which we perturb.

2. The focusing errors in the part of the arc which we perturb. Such errors can reduce the effects from the harmonic perturbations, as was shown in section II.10 of the above collider note, especially in the case of systematic errors.

\section{VI.4.2 Separation of Horizontal and Vertical Betatron Frequencies}

We show in Figs. 22a,b the vertical phase-shift produced by a systematic regular focusing deviation of 0.0035 of the main field, and of opposite sign for the focusing and defocusing magnets*. In Fig. 22a, a negative perturbation has been induced in the focuping magnets, and in Fig. 22b, a positive perturbation.

As can be seen, the variation of the vertical phase is positive in Fig. 22a, and negative is Fig. 22b, for the part of the arc where the perturbation has been applied (units 1700 to 2400 ). The cumulative phase-shift is about $80^{\circ}$, which is close to what is expected for a relative perturbation of $\mathbf{0 . 0 0 3 5}$.

\section{VI.4.3 Other Perturbations and Empirical Adjustments}

As shown in chapter IV of the colliaier note above, the skew focusing perturbation are too weak to have significant effects. We have however used the full set of pert urbations to tune, the lattice on the one hand, and the spots at the entrance to the final focus section on the otber hand, in an empirical way. We give exemples of this below.

We show in Figs. 23 and 24 a full set of betatron oscillations, horizontal and vertical, with their couplings in the opposite plane, and for four initial phases separated by $\pi / 4$. These oscillations were measured after empirical adjustments of the harmonic correctors.

* The phase-shift is calculated in the following way ${ }^{24}$ : By noting that consecutive mesurement points are separated by the phase-shift per cell, which is $\mu=108^{\circ}$, and by considering that a weakly perturbed osciflatory mouvement can be represented by letting the phase and the amplitude of the oncillation rary slowly, one can solve, for each couple of consecutjue dats points $\left(x_{n}, x_{n+1}\right)$, the system:

$$
\left\{\begin{array}{l}
x_{n}=a_{n} \cos \left(n \mu+\Delta \phi_{n}\right), \\
x_{n+1}=a_{n} \cos \left((n+1) \mu+\Delta \phi_{n}\right),
\end{array}\right.
$$

where $a_{n}$ and $\Delta \phi_{n}$ represent the variable amplitude and phase in question. An exact calculation would also give terms proportional to the derivatives of $a$ and $\phi$. Since we congider that these functions are slowly varying, we can neglect these terms. 
As can be seen, they are essentially regular.

We show in Figs. 25a,b the beam shape on a phosphor screen, photographed before and after adjusting it empirically. The two sets correspond to two different experiments, where the optical parameters of the beam at the entrance to the arc were different. In both cases, it was possible to adjust the harmonic correctors to cancel the variations.

\section{VI.4.4 Conclusions from the First Tests}

These first tests have shown the feasibility of the harmonic correction method which we have proposed, have contributed to improve the optical transfer of the arcs, and given the system an additioral "knob", upstream of the final focus section, to adjust the matching of the phase-space there.

These tests have also shown that the effects are weaker the predicted, in most part because of systematic errors and because of the rolls along the part of the arc where the harmonic correction system is installed. To obtain larger effects, the current of the power supplies feeding the correction windings would have to be raised, by a factor of two or three. 
(a)

(d)
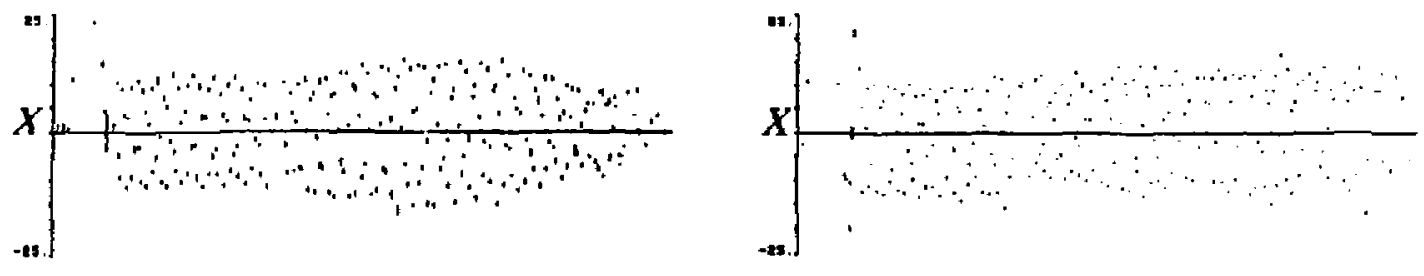

(b)
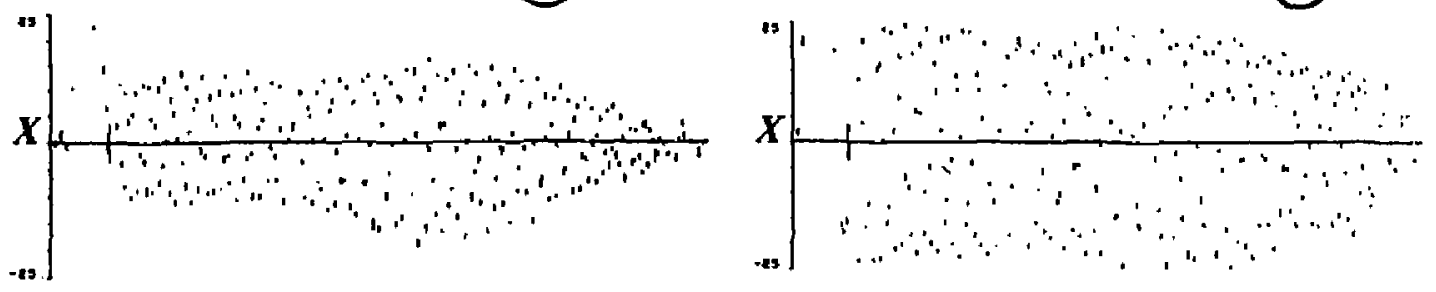

(c)
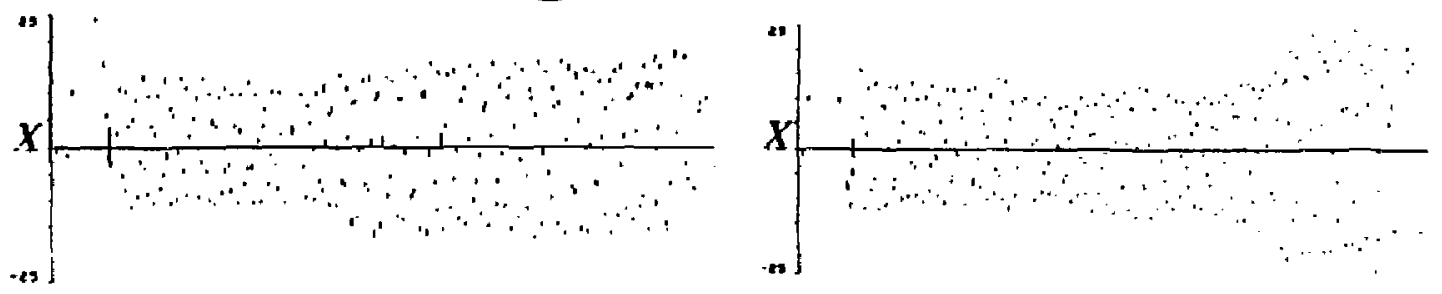

Figure. 20 Fescnant growth of the horiznntai betatron oscillations, from a focusing perturbation at twice the betatron frequency. 

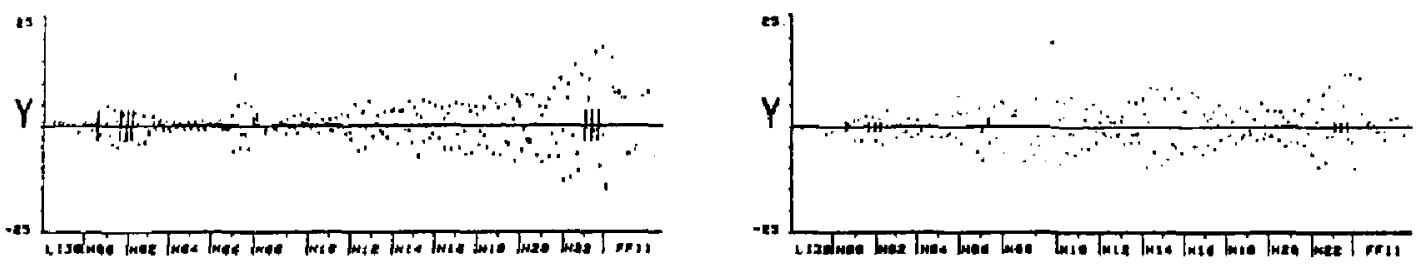

(b)
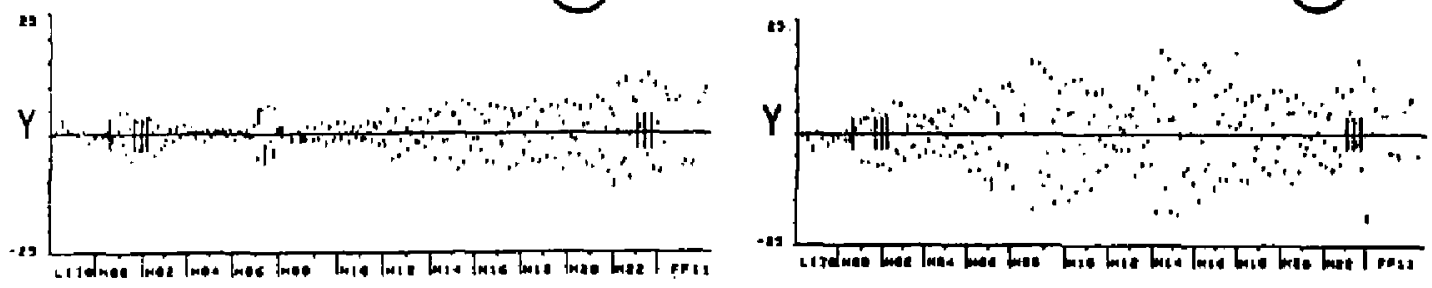

(c)
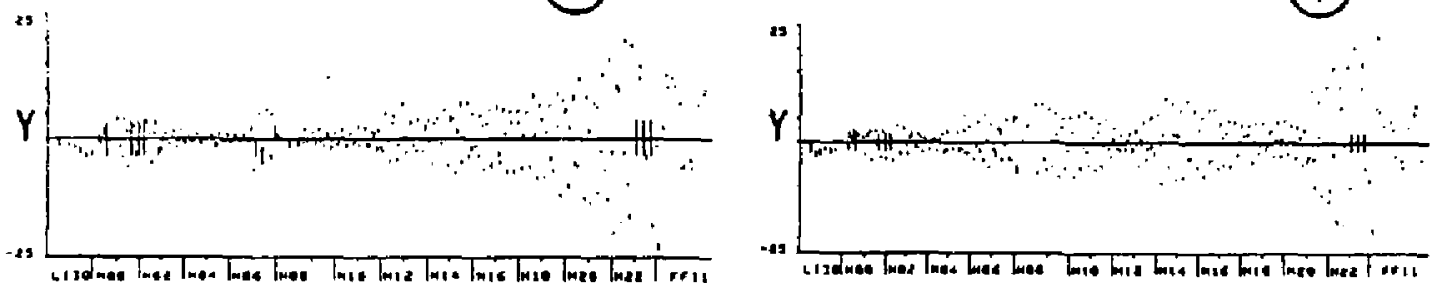

Figure. 21 Coupling into the vertical plane corresponding to the oscillation in figure 20. 

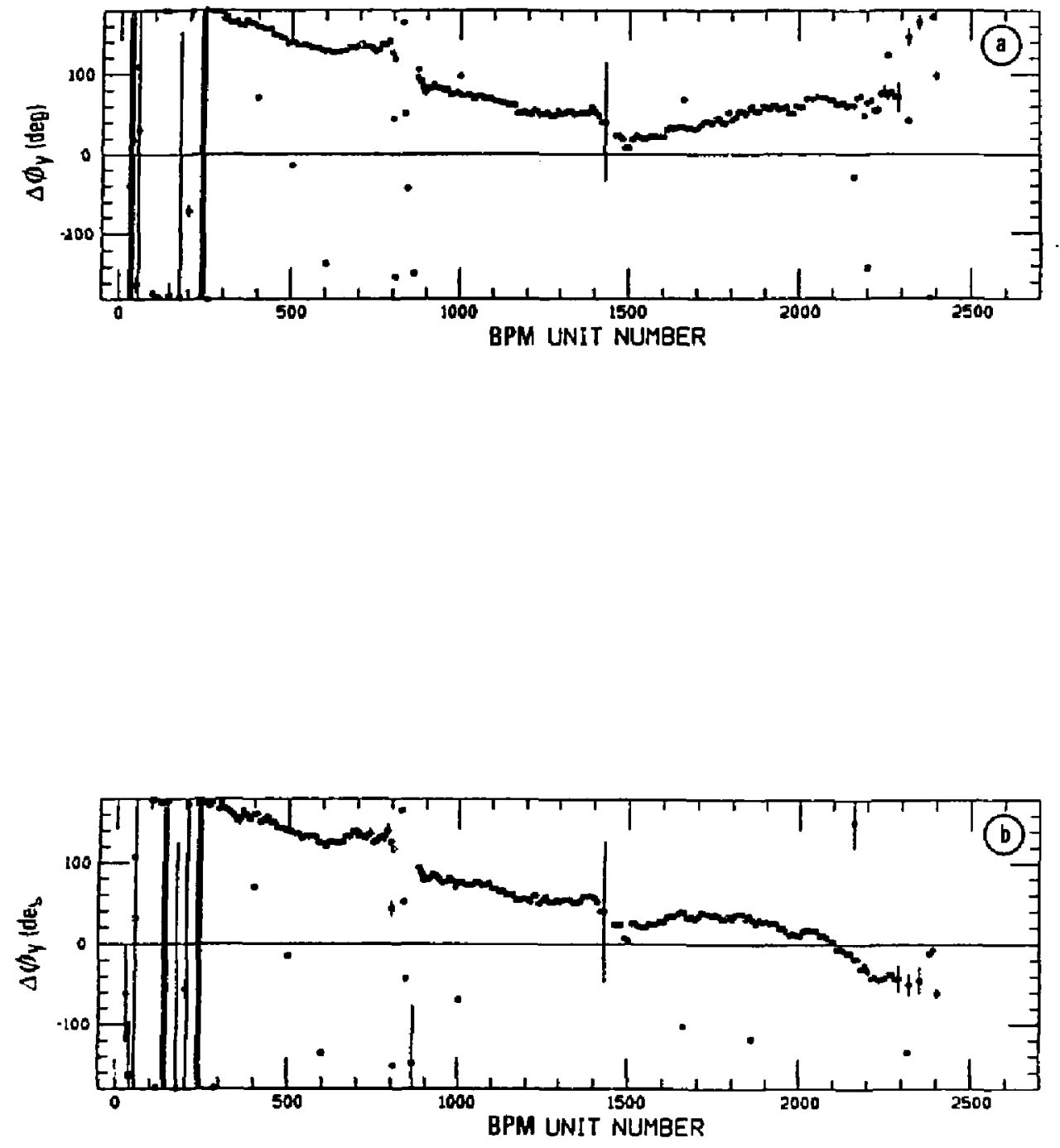

Figure. 22 Shift in vertical betatron frequency from systematic perturbations of focusing and defocusing magnets with opposite signs. 

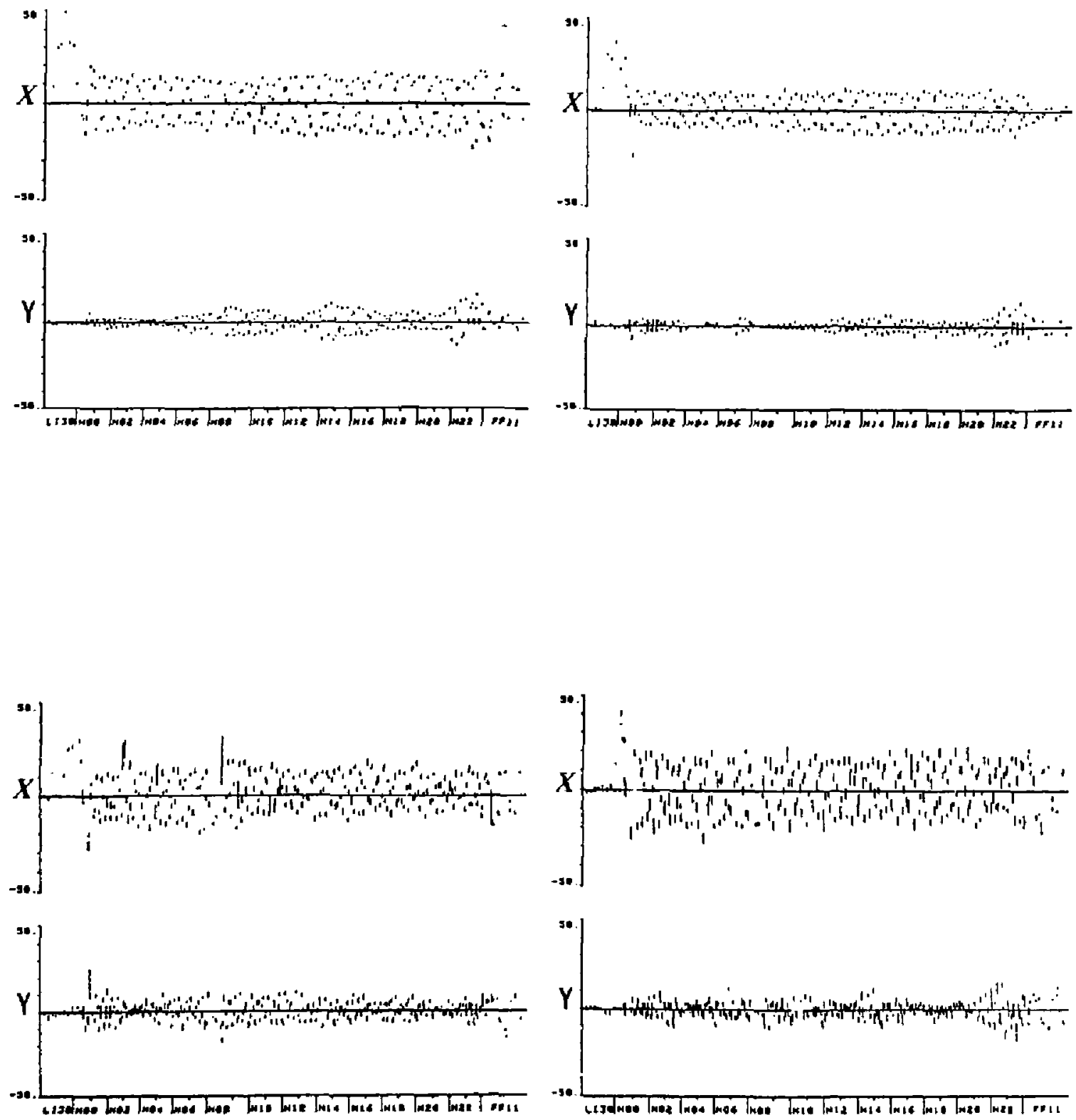

Figure. 23 Transfer of horizontal betatron oscillations, and coupling into the vertical plane, for four initial conditions corresponding to phases separated by $45^{\circ}$, after optimization of the harmonic correctors. 

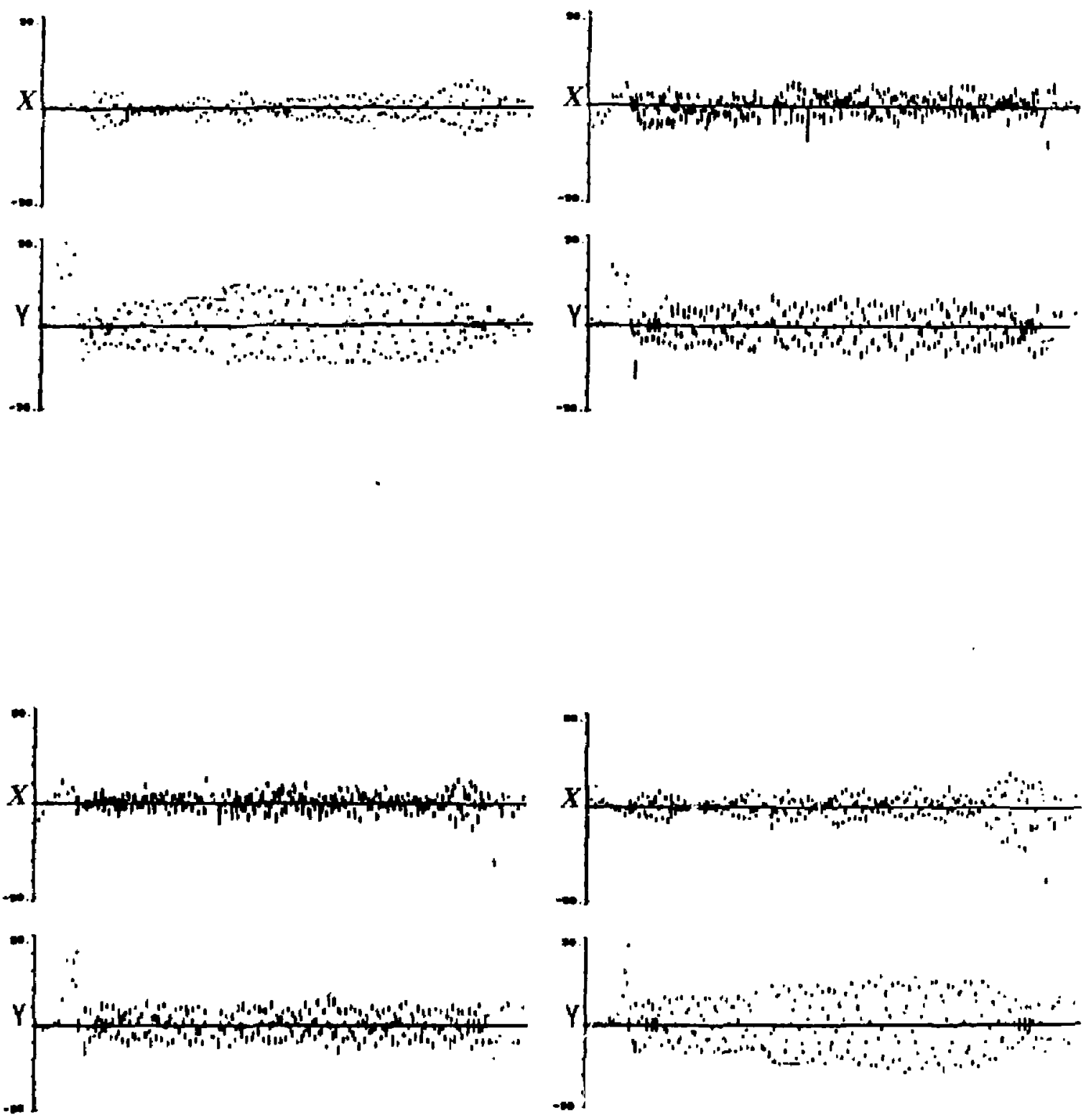

Figure. 24 Transfer of vertical betatron oscillations, and coupling into the horizontal plane, for four initial conditions corresponding to phases separated by $45^{\circ}$, after optimization of the harmonic correctors. 


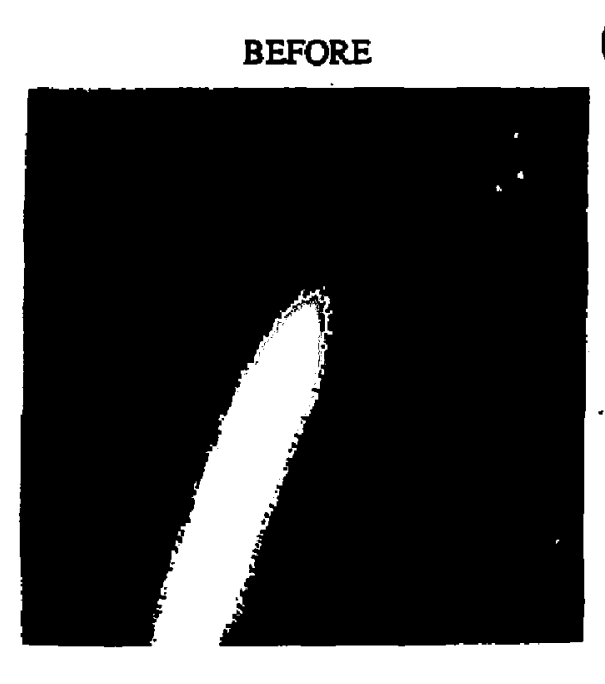

a)

\section{AFIER}

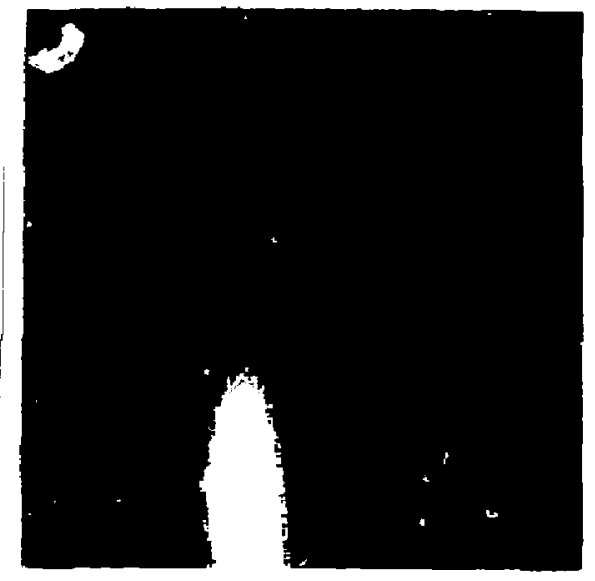

BEFORE

(b)

\section{AFTER}
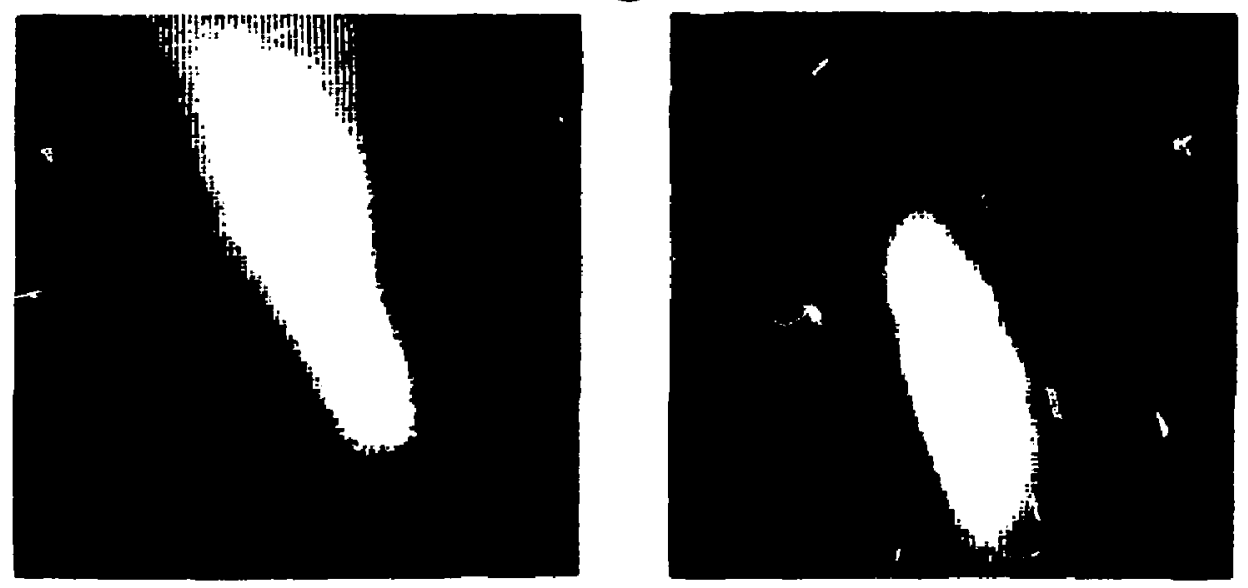

Figure. 25 Empirical adjustments of the beam size at the end of the arc, on two occasions ((a) et (b)). The photographs on the right show the beam before correction, and those on the left the beam after correction. 


\section{REPORTS AND PUBLICATIONS ON OPTICAL CORRECTIONS IN THE FINAL FOCUS}


VII.1 "Orthogonality of Final Waist Corrections at the IP of the SLC"

The optical corrections in the final focus are all strongly coupled except for the three final adjustments to position the waists at the interaction point, which can be made fully orthogonal. This enable to simultaneously correct and measure phase-space parameters at the interaction point. The orthogonalization is shown :n this collider note, together with a summary of the experimental algorithm. 


\title{
SINGLE PASS COLLIDER MEMO $\quad$ CN-369
}

\author{
AUTHOR: Philip Bambade $\quad$ DATE: October 27, 1988
}

TITLE: ORTHOGOVALITY OF FINAL WAIST CORRECTIONS IT THE IP OE THE SLC*

\section{INTRODUCTION}

Because the SLC finai IP spot is produced by an aberration-dominated optical system ${ }^{1}$, all components and couplings between dimensiors of transverse phase-space must ${ }^{2}$ be controlied in the experimental tuning algorithm. For equal emittances $\epsilon_{z}=\epsilon_{y}$, this amounts to ten linear optics adjustments ${ }^{\dagger}{ }^{\dagger}$. These adjritments are coupled and depend non-linearly on phase-space parameters. A ten-dimensional non-linear fitting pre gram ${ }^{3}$ is therefore used to match the lattice in the Final Focus to the input beam. Local orthogonal "knobs" are also defined for fine-tweaking around the initial solution, although this is not always practical because of steeting from the ienses.

The three final yaist corrections ${ }^{4}$ are however fully orthogonal to the other seven optical adjustmen:s. This means that they do not cause any of the other seven optical distortions. We refer to this as external orthogonality.

They can also be made internally orthogonal. This means that each one of the three orthogonalized controls can be applied independently of the two others. It also allows one to simultaneously correct and determine the phase-space at the IP.

"Work aupported by Department of Energy Contract DE-AC03-76SF00515.

t In the case of equal emittances these ten adjugtnients are grouped ${ }^{2}$ in three sets:

1. Four corsections to minimize the spatial an J angular disperaion in boch planes,

2. Three corrections to the betairor, angular apread at the IP, by controlling the maynitude of $\left\langle x^{\prime \prime}\right\rangle$ and of $\left\langle y^{\prime \prime}\right\rangle$, and by minimising the $\left\langle x^{\prime} y^{\prime}\right\rangle$ correjation, and

3. Three adjustmencs to ,osition the waists in both planes at the IP, 'Jy minimizing the correlations between the positions $x, y$, and the angles $x^{\prime}, y^{\prime}$ in both planes.

The ten variable quadrupoles used tor these conections are shown in Fig. 1. Because each correction is coupled to the ones downstrean, they mut be applied sequeatially. A flow diagram illustrating this sequentiäl application is shown in Fig. 2. 
Both have been demonstrated to work experimentally ${ }^{5,6}$, and form the basis for the on-line tuning algorithm?. In this note, we show the orthogonality of the corrections and outline the experimental procedure and its limits.

\section{EXTERNAL ORTHOGONALITY OF WAIST CORRECTIONS}

The three final waist corrections are designed to cancel the correlations between the positions $x, y$, and the angles $x^{\prime}, y^{\prime}$ in both planes at the IP. The $\left\langle x x^{\prime}\right\rangle$ and $\left\langle y y^{\prime}\right\rangle$ correlations" are minimized by combining trim windings in two regular quadrupoles of the Final Triplet, one defocusing (QD2B), and one focusing (QF3). We refer to them as the in-plane waist adjustments. The $\left\langle x y^{\prime}\right\rangle$ correlation, which at the waist and for $\epsilon_{z}=\epsilon_{y}$ is equal to $\left\langle y z^{\prime}\right\rangle$, is minimized with a single skew quadrupole (SQ3) just upatream of the Final Triplet. Thio one is referred to as the out of plane waist adjustment. The correction elements are indicated in Fig. 1.

These final adjustmenta are normally decoupled from disporsion correction: because the dispersion is nominally zero in the Final Triplet.

They are also close to decoupled from the angular spread corrections. To see this, we consider for eimplicity an uncorrelated $\left(\left\langle x x^{\prime}\right\rangle=0\right)$ beam focused to a waist by a thin lens in the horizontal plane. From linear optics, we compute (to first order) the variation of the spatial beam size $\sigma_{x}$ and of the angular beam size $\sigma_{x^{\prime}}$ as a result of varying the strength of the lens:

$$
\left\{\begin{array}{l}
\sigma_{2}^{2}\left(\delta_{Q}\right)=\sigma_{2}^{2}(0)+\delta_{Q}^{2} \sigma_{Q}^{2}, \\
\frac{\Delta \sigma_{x^{2}}}{\sigma_{z^{\prime}}} \simeq \frac{-\delta_{0}}{1+\left(\frac{\delta_{0}}{\sigma_{Q}}\right)^{2}} .
\end{array}\right.
$$

In (I), $\sigma_{Q}$ is the beam size at the lens and $\delta_{Q}$ is the fractional strength variation of the lens.

For $\sigma_{Q}$ large compared to the minimum beam size $\sigma_{2}(0)$, small adjustments are sufficient to change the size at the waigt significantly, with only a small perturbation to the angular spread. At the Final Triplet, $\sigma_{Q}$ is typically a thousand times larger than $\sigma_{z}(0)$. The quadrupole adjuatments need thezefore be only a few percent. Over that range, the change in angular spread is negligible.

Thus the three final waist adjustments are essentially orthogonal to the other seven optical corrections. The opposite is not true: the other seven corrections

- In Transporto notation, $<I x^{\prime}>=\sigma_{2 x},<y y^{\prime}>=\sigma_{43}$, and wo on... 
are $s_{i}$ ongly coupled to the three final waist adjustments. The experimental tuning algorithm is therefore sequential. It is summarized in the flow diagram in Fig. 2.

\section{INTERNAL ORTHOGONALITY OF WAIST CORRECTIONS}

We begin by considering the in-plane waist adjustments (i.e. the minimization of the $\left\langle x x^{\prime}\right\rangle$ and $\left\langle y y^{\prime}\right\rangle$ correlations). A regular lens perturbs each plane proportionally to the beam size at the lens in that plane. Since this beam size is naturally larger in each lens in the plane in which it is focusing, QF3 and QD2B can be combined to control the horizontal and vertical waists independently. The coupling coefficients, found using TRANSPORT ${ }^{9}$, are:

$$
\left(\begin{array}{l}
\Delta f_{z} \\
\Delta f_{y}
\end{array}\right)=\mathrm{C}\left(\begin{array}{c}
\delta_{Q 3} \\
\delta_{Q 2 B}
\end{array}\right), \text { with } \mathrm{C}=\left(\begin{array}{cc}
-1.89 & 0.70 \\
0.80 & -1.37
\end{array}\right)
$$

In (2), the fractional quadrupole strengths $\delta_{Q 3}$ and $\delta_{Q 2 B}$ are in parts per thousand, and the longitudinal waist motions $\Delta f_{x, y}$ are in centimeters.

The couplings in $\mathrm{C}$ are close to independent of input mismatch. This is evident for a mismatch of the IP angular spreads from the form of (2). It is also true in the case of a largely correlated input phase-space into the Final Triplet (in-plane correlations), which the waist corrections are designed to cancel. This can be seen by considering the nominal IP phase-spare obtained after the correction, and by back-tracking the beam into the lenses: since the changes in the strengths of QF3 and QD2B required by the correction are small, the beam sizes are perturbed negligibly in the lenses. Since their effect is proportional to this beam size, the couplings in $\mathrm{C}$ do not change significantly. The linear combinations defined in (2) can thus be used for orthogonal control independent of the mismatch of the input beam phase-space ${ }^{*}$.

In the case of the out of plane waist-adjustment, (the $\left\langle x y^{\prime}\right\rangle$ and $\left\langle y x^{\prime}\right\rangle$ correlations) using the skew lens SQ3, orthogonality to the in-plane corrections is obtained by requiring an upright beam shape near the Final Triplet (i.e. <

* This is not the case for the other optical adjuntments in the Final Focnu, where the relative settings munt be calculated through a non-linesr fitting programs and depend thus on the initial condition. Since tbi initial condition muat be obtained from meanremente with possibly large errors, the calculated solution can be signifirantly of $\mathbf{a}$ s reault. The correction can in thi care require several iterations. This in sapecially the cae for the angular spread corrections. Operationally, some improvement is oblained by besiag the calc ${ }^{-1}$ ations on time-averaged quantities. 
$x y>=0$ ). Operationally, this condition is obtained by observing the beam on a profile monitor (ST4) near the Final Triplet, and by adjusting a second skew lens, SQ17.5, located in the First Telescope, which is part of the angular spread corrections.

Wo show this by the following thin lens argument ${ }^{10}$. Consider two lenses, one regular and one skew, of strengths $K$ and $S$ respectively, with parailel to point focusing to the waist. The change in angular apread at the waist is in this case strictly zero. The combined effect of the two lenses on the beam is:

$$
\sigma^{\text {out }}=R \sigma^{\text {in }} R^{t} \text {, with } R=\left(\begin{array}{cccc}
-K & 1 & -S & 0 \\
-1 & 0 & 0 & 0 \\
-S & 0 & K & 1 \\
0 & 0 & -1 & 0
\end{array}\right) \text {, }
$$

where $\sigma_{\text {ous,in }}$ are the beam matrices' describing the four-dimensional phase-space at the waist and in the lenses. From (3), the beam-size at the waint is, in the horizontal plane:

$$
\left(\sigma_{x}^{\text {out }}\right)^{2}=\sigma_{x^{\prime}}^{2}+\sigma_{x}^{2} K^{2}+\sigma_{y}^{2} S^{2}-2<x x^{\prime}>K-2<y x^{\prime}>S+2<x y>K S,
$$

where the subscript in has been omitted on the right hand side. The coupling between the two corrections cancels if the correlation $\langle x y\rangle=0$. This has been verified independently in two simulations ${ }^{11,12}$ of the optical corrections for the Final Focus Systers.

IV. EXPERIMENTAL PROCEDURE AND PHASE-SPACE DETERMINATION AT THE IP

After the $\langle x y\rangle$ correlation has been minimized at ST4, by adjusting the skew lens SQ3, the cut of plane waist adjustment $S Q 3$ and in plane controls $\Delta f_{z, y}$ defined in (2) form an orthogonal set of correctors. For minimization of the IP spot, they can therefore be applied independently and in any order.

From (4) or from (1), we see that the beam sizes depend parabolically on the controls. This enables one to find the optimal corrections by symmetry even if the minimum of the pare ola is not resolved instrumentally. This minimum is not resolved for beam sizes smaller than the carbon flament target ${ }^{12}$ used for diagnostic purposes (there are three wires, with diameters of 4,7 , and 20 microns). 
In order to simultaneously measure the phase-space parameters at the IP, wc first apply the SQ3 correction, to minimize the otst of plane correlations. After this, the beam sizes at the IP can be written as a function of the in-plane Final Triplet orthogonal controls $\Delta f_{x, y}$ as:

$$
\sigma_{x, y}^{2}=\epsilon_{z, y} \beta_{x, y}+\frac{\epsilon_{x, y}}{\beta_{x, y}} \Delta f_{x, y}^{2}
$$

where $\epsilon_{x, y}$ and $\beta_{x, y}$ are the emittances and the $\beta$-functions in each plane respectively. Fitting a parabola to each measurement gives $\epsilon_{x, y}$ and $\beta_{x, y}$. Although the angular spreads $\sigma_{x_{1} y^{\prime}}=\sqrt{\frac{f_{s, x}}{\theta_{x, y}}}$ are well determined from the branches of the parabolas, the minirnum linear beam sizes $\sigma_{x, y}=\sqrt{\epsilon_{x_{1}, \beta_{2, x}}}$ are not if the minimum of the parabola is not resolved. This lack of resolution can occur because of the finite wire-target size mentioned sbove, and because of the following optical reasons:

1. For unequal emittances, because the correlations $\left\langle x y^{\prime}\right\rangle$ and $\left\langle x^{\prime} y\right\rangle$ are not ${ }^{8}$ necessarily equal, there may be residual uncorrected crosse-plane coupling terms in the spot.

2. Before full implementation ${ }^{3}$ of the seven other optical corrections, the size of the third order chromatic aberrations can dominate ${ }^{5}$ the linear component of the beam size at the IP ${ }^{\star}$.

In both cases, the linear variables $\beta_{z, y}$ and $\epsilon_{z, y}$ will be over-eatimated. In order to relieve the effects from 2., a detuned optical configuration, with purposely small angular spreads, can be used, such that the third order aberrations are negligible compared to the linear beam size. For the resson, such a configuration, with $\beta_{x, y}=3$ centimeters, instead of the nominal $\beta=0.75$ centimetern, was used in the initial commissioning phase.

Effects from 1. cannot be haviled in the Final Focus with the present correction scheme, which is designed for equal emittances in both planes. Operationally, it is therefore desirable to maintain equal emittances throughout the upstrcarn parts of the SLC.

- The second order aberrations muat aleo be eancelled by fitting the chromatic correction sextupole after each siynificant optical adjuntment, to take into eccount the deviations in the lattice caued by the optical matebing. This sewule from the fect that the six quadrupoles used to conect the betatron phase-epece atraddlo the chromatic correction. 


\section{ACKNOWLEDGEMENTS}

I wish to thank K. Brown, A. Hutton, J. Hailssinski and W. Kozanecki for useful comments on this note. In addition, I would like to acknowledge the contribution of $\mathrm{D}$. Ritson, with whom I collaborated on these problems.

REFERENCES

(1) "SLC Design Handbook" (December 1984).

(2) P. Bambade, "Beam Dynamics in the SLC Final Focus", SLAC-PUB-4227 (Jure 1987),

(3) C. Hawkes and P. Bambade, "First Order Optical Matching in the Final For:us Section of the SLAC Linear Collider", SLAC-PUB-4621 (May 1988)

(4) J. Murray et al., "The Completed Deaign of the SLC Find Focus System", SLAC-PUB-4219 (February 1988)

(5) P. Bambade, "Recent Progreas at the SLAC Linear Collider", SLACPUB-4610 (April 1988)

(6) P. Bambade et al., "Operational Experience with Optical Matching in the SLC Final Focus Syatem", To be publiahed at the Particle Accelerator Conference, in Chicago (March 1989).

(7) N. Phinney et al., "An Automated Focal Point Pooitioning and emittance Measurement Procedure for the Interaction point of the SLC*, To be published at the Particle Accelerator Conference, in Chicago (March 1989).

(8) P. Bambade, "Number of Dimensions of Optical Correcticn Space in the SLC Final Focus System", Collider Note in preparation.

(9) K. Brown et al., "TRANSPORT", SLAC-91, Rev. 2 (May 1977).

(10) This argument was suggested by $D$. Ritson.

(11) D. Ritson and P. Bambade, private simulation.

(12) R. Servranclox and K. Brown, private aimulation.

(13) C. Field et al., "High Resolution Wire-Scanner for Micron Size Profile Measurements at the SLC", SLAC-PUB-4605. 

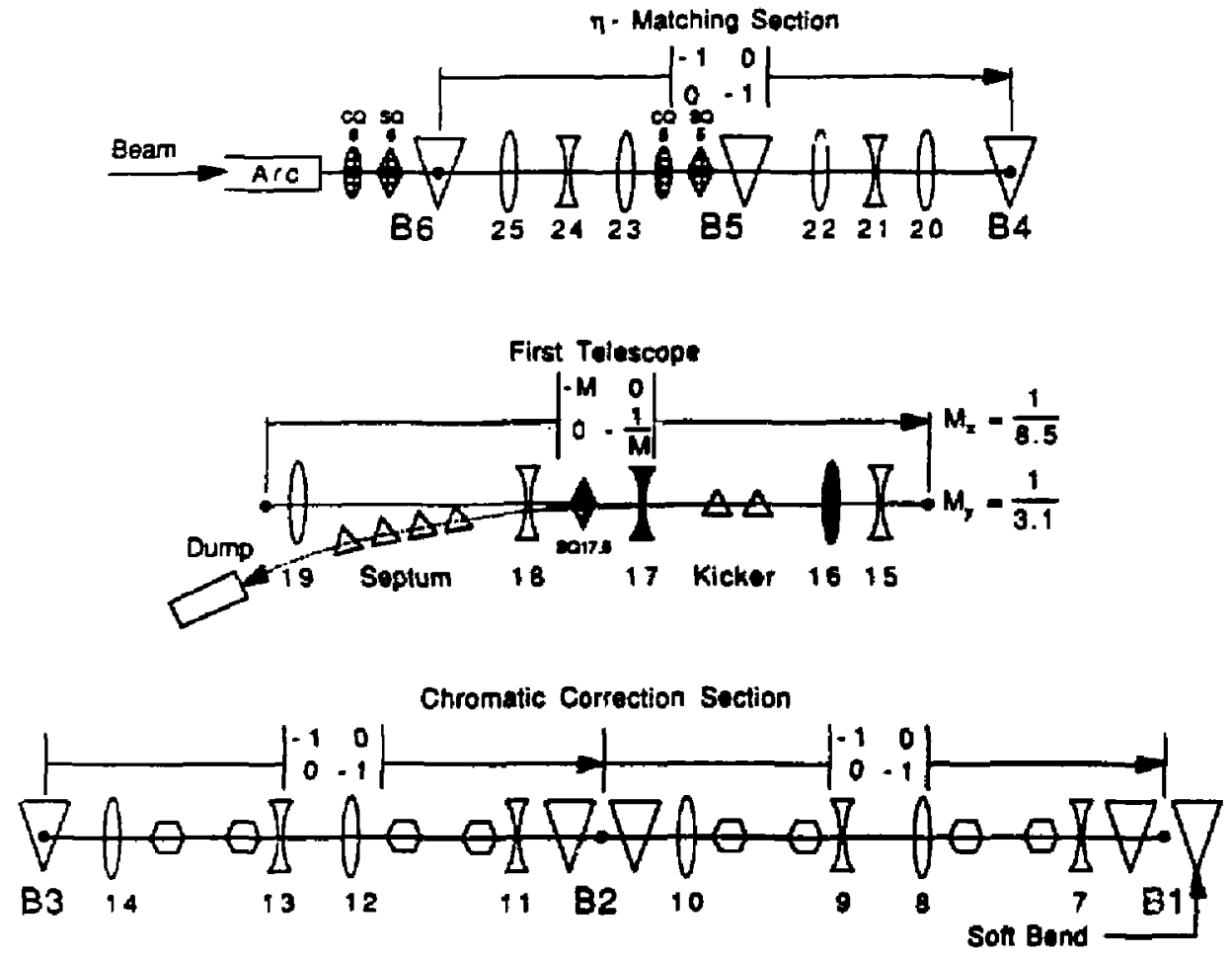

Final Tuloscope

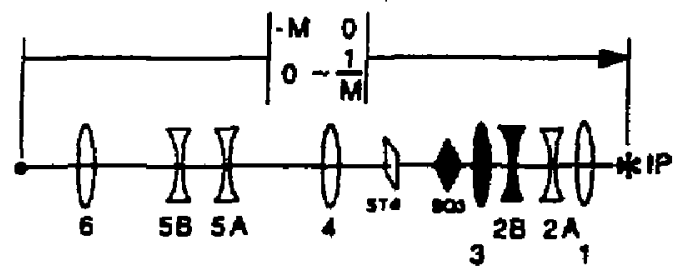

wases

Fig. 1: Schematic of the SLC Final Focun Syatem optica. The six variable quadrupoles used for adjuating the betatron phane-apace are ahown ahaded. The four quadrupoles used to correct the dispernion function are shown crons-hatched. The profile monitor ST 4 is located immediately upatream of the akew quadrupole SQ3. The sequential application of these ten adjustments is summarized in the flow-diagram in Fig. 2. 


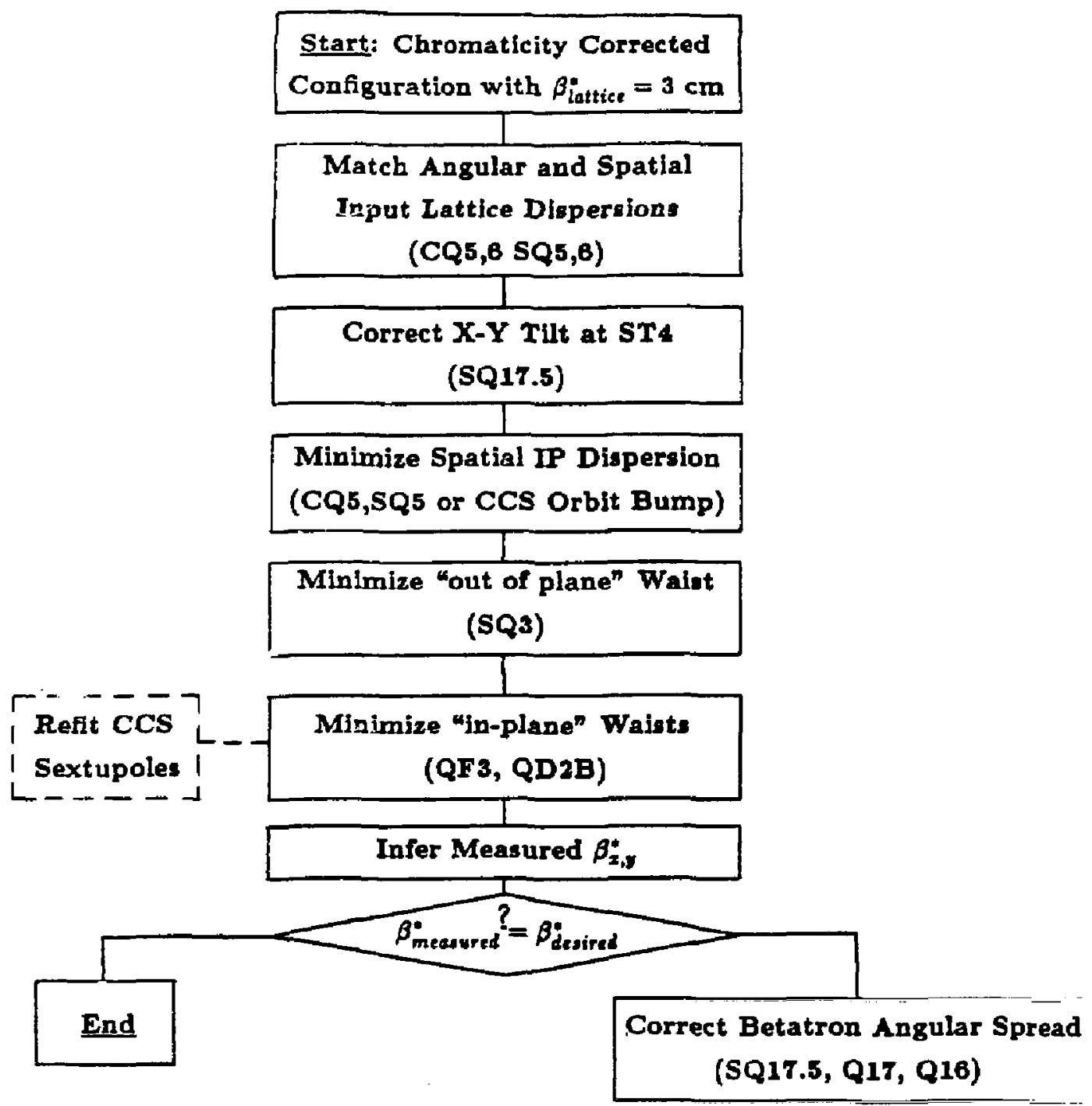

Fig. 2: Flow-diagram summarizing the ten linear optics adjustments required to minimize the beam size at the IP of the SLC. These adjustments are grouped and applied sequentially as shown in the boxes. Some iteration of the waist and IP dispersion minimization is usually performed. In addition, the sextupoles are refitted after each significant wist correction. The main correctors used at each step are indicated in parenthesis. The full system is shown in Fig. 1. 
VIL2 "Beam Dynamics in the SLC Final Focus System"

This conference article describes the initial development of the experimental tuning algorithm, enabling to adjust the final focus for maximum luminosity, given a perturbed input phase-space. The correction algorithm had to be designed pragmatically and given the already built final focus beam-line. It is based on the general framework of the optical design of the system. The paper gives an overview of the general commissioning strategy for the final focus. It also provided the basic groundwork for the specification of the on-line computer control and modeling of the final focus, and served as a building block towards further work on this subject. 


\author{
P. 5. DAMBADE
}

Slanjord Linear Accelemer Center

Stanjord Univeraily, Stanford, California o,sos

\begin{abstract}
Abotract
The SLC luminosity' is reached by colliding beams foeussert to about 2 ans transverte size. The Find Focus Syotem (FF5) must ensbite, byond ite bevic optical design, to the de tection and correction of error mecurulated in the rytam. In

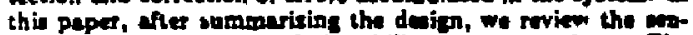
sitivity to anch emors and the ability to cosnet them. The overall cuning existary involve throt phase: single beam mot minimistion, atering the bame in colliaion and inminodity optimization with bevorben sfich.
\end{abstract}

\section{Summary of Optleal Dealen 1 ,}

Focunint the bean to amull trenovered dize would be eary if the input phwe ipece (tranverwe emittance and entery opread) ware imall enouch. A monetnarretie beam with hori. zoned emintance c, = of" of would, for exemple, be focuend to $\sigma_{z}^{\prime}=\sigma_{0} / \cdot / L$ a dinunce $I^{\prime \prime}$ from a lens with focal longth $\| /=1 / l^{*}+1 / L$ (see Fig. 1 ). In a more realiatic beam with frite energ spread oc, rays on the edge of the energ diatribution are focuased at an axial ponition diapleced by $f^{\circ} \sigma \mathrm{s}$, thus adding $2 l^{\prime} \circ \mathrm{g}^{\circ}$ to the overall size (- fector 2 is put in sinet $\mathrm{st}$ least iwo lenes are uned to focus both plane). This ehromatie aberrotion is negligible if $\cos _{\mathrm{z}} \leq \sigma_{\mathrm{z}}^{2} / 2 l^{*}$. In the SLC where

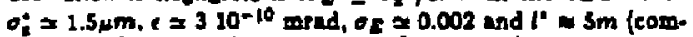
puted to the principle plane of the ind lantea), it amounts to osirem $=1 \mu \mathrm{m}$, and thu doranates the apot.

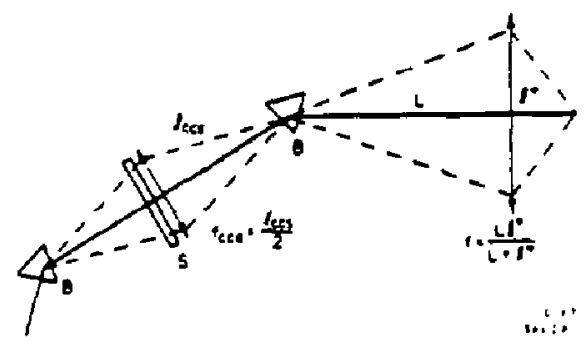

Fig. 1. A simplified Fian Focus.

The resulting luminoaily, compuled by averaging the unual expresion owet the two beams' energ diatributione (asumed square with helf-width $\sigma_{\Sigma}$ ), is given by:

$$
\mathcal{C}\left(\theta^{\circ}, \sigma_{E}\right)=\frac{\int N^{2}}{2 \pi \sigma_{E}^{2}} \int_{a}^{\infty} \int_{0}^{\infty} \frac{d \sigma_{E}^{+} d \delta_{E}^{-}}{2 \sigma^{0}+\frac{1}{\delta F^{2}}\left(\delta_{E}^{+2}+\delta_{E}^{-2}\right)}
$$

where $F=0 ;\left(\delta_{E}\right) / 0 ; \sigma_{z}$ is a measure of the sberration. It is shown versus $\beta^{\circ}$ in Fig. 2, nortnalised to $\mathcal{C}\left(\beta_{n}, \sigma_{C}=0\right)$. lor an a-built FFS with no chromatie compenstion $(F=15 \mathrm{~m}$ ). lndependent of $c$, it tives the optimum $\rho^{*}$ for wuch an FFS. For $\sigma_{E}=0.002$ and $\beta_{i x}=1.5 \mathrm{~cm}, C$ is down by a factor 3.5. This is not a low 18 what we sel directly with the size etimale because the aberration epreads the edges of the bunch out more than its core, and because the luminanity is a sum of iquare.

\footnotetext{
- Work supported by the Department of Enercy, contract DE-ACO3-T6SFOOSis.
}

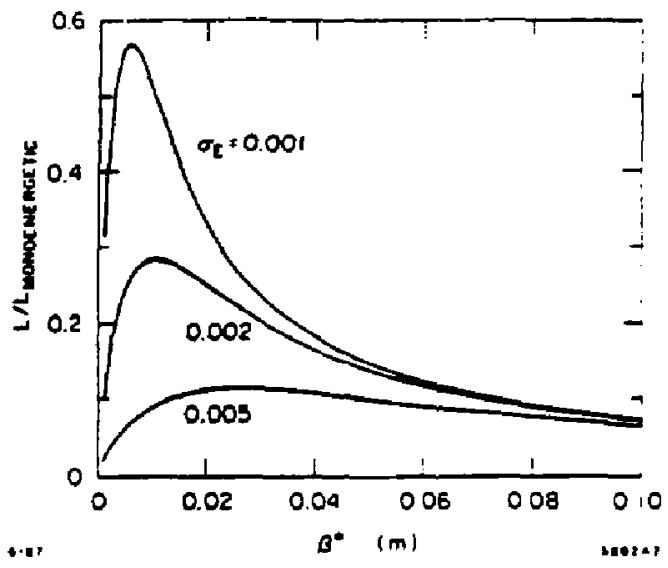

Fle. 2. Raletive luminosity lows vortus $\beta^{*}$ and $\sigma_{E}$ for $\&$ Find Foew without chromutic correction.

Compensation require apecial Chrometic Correction Section (CCS) upstren of the End lens. In a simplified CCS, two dipole of etrength $B$ eaparated by $2 l_{\text {cect }}$ ere imaged by qued with focel length $t_{\text {sed }} / 2$, noulting in 211 order achro mat (ase Fis. 1). Near the quad is a extupole of atrength $S$, in which the beld verie quadratically with excuraion, and in which rays with anergy deviation of are trasuported of axil. This produce a otronger ovarall quad for says both of.

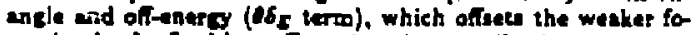
cussing in the fad lons. Equating the contributions to ocen from the final lens and from the sextupole (neglestine the CCS qued), we find that $M=l^{*} / L, R_{1}=l^{*} / l_{\text {eca, }}^{2} B$ and $S$ acale like $S \propto R_{1} / M B$.

Unfortunalely, the sextupole wo deflecte raye solely orfenergy or off-angle, thus tiving teras in $t^{2}$ and $\delta f$. Cancellins these two new aberrations require that the CCS be made of two consecutive and identical sections, with eextupoles in peirs 7 phese shift apart and sequential symmetry for the $\eta+$ [unction. The real syatem, ${ }^{2}$ designed to focus achromatically in both planes, usea telescopes, exch consiasint of two triplets. instesd of the lenses. This minimizes.s the dominant of $\sigma_{E} \phi \delta_{E}$ terms and suppreses the other $2^{\text {ad }}$ order term in $I \delta_{E}, y_{E}{ }_{E}$ while demagnitying in both planes. The chromasic correction, also done in both plane, requires two extupole families. Coupline effect between them can significently enhance $3^{\text {rat }}$ order chromatic and geometric contributions and must be minimized. The three dominast terms are $\theta \delta_{E}^{2}, \theta^{2} \delta_{E}$ and $A^{3}$. Feglecting the

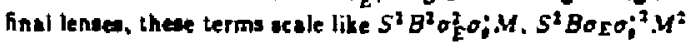
and $S^{2} \sigma^{3} M^{3}$. Substituting for the sextupole strength needed

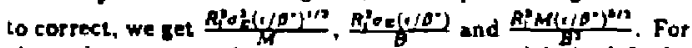
eiven phese-space voiume, spece constraints and desired $g^{*}$ the overall effect of these aberpations is minimized adjusting M.B and $S$ to balance them out. An upproximate criterion ${ }^{1,2}$ is abtained equating the two $1^{\prime \prime}$ terms giving of" $=\sigma_{E} B$. Physically this means that monochromatic and chrornatic sizes should be about equal in the sextupoles. 
These considerations are relevant not only to the design, but ss we shall see, to the experiment of tuning strategy: We must in effect insure proper optical matching into the CCS to maintain the optimization. The whale system is shown in Fig. 3. It includes two mare sections to mintch the Are lattice.
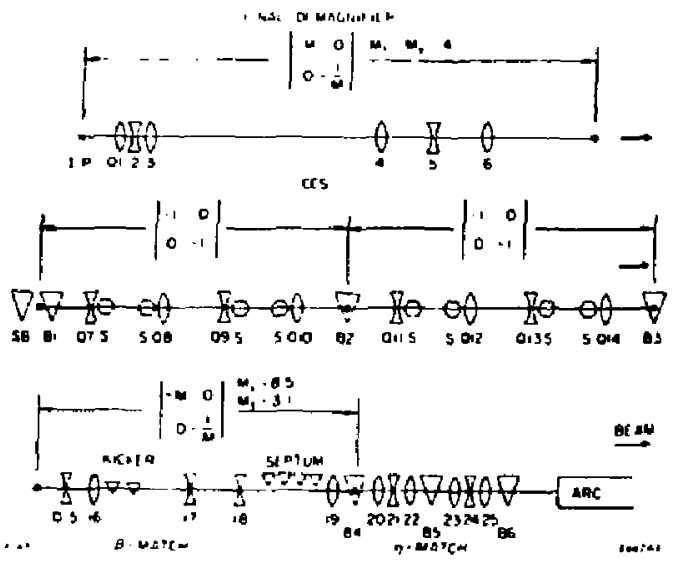

Fig. 3. Schematic of FFS.

\section{Sensitivity to Optical Mismatch}

In real operation, both the volume and shape of the injected whase space differ from spexification because of ertors, thereby inereasing $a^{\circ}$.

Enhancements in t, from wakefields in the Linac or synthrotron radiation in the $A r c$, as well as an imperfectly miniInized $a_{E}$, are vncorreclable in the FFS. The damage can, how=ver, be reduced somewhat by retuning the betatron motch into the CCS. For larger $e_{1} \sigma^{\circ}$ is first dominated by the s'd $^{\text {rotder }} \theta^{2} \delta_{E}$ term. Varying $\beta^{*}$ to minimize the overall $\left.: \beta^{\prime}+\left(\sigma_{E} c / \beta^{\prime}\right)^{2}\right)^{\prime}$ size gives $L \alpha \epsilon^{-5}$ for $\beta_{o p 1}^{\prime} \alpha d$. Similarly or $\sigma_{E}$ (now with $\delta_{E}^{2} \theta$ ) we would get $L \propto \sigma_{E}^{-2}$ for $\beta_{o p t}^{*} \propto \sigma_{E}^{2}$ ir he bunch remained gaussian. Actual simulation (MURTLE') hows close to linear loss with weak dependance on $\beta^{*}$ (see his. 1).

Oplical distortions from gradient errord upstream are nostly linear" and can be corrected within come bounds. Ihe primary effect results from $1^{\prime \prime}$ set enhancing $a^{*}$ diectly by correlating positions with angles or with $\delta_{E}$ at the P. This amounts to axial offsets in the waists, $x-y$ coupling I $\phi$ or $y \theta$ terms) and anomalous $\eta_{x, y}$. The axial woist offet $A_{\text {waict }}$ must be corrected to better thas $\beta^{\prime}=0.75 \mathrm{~cm}$, since $4_{\text {eff }}^{*}=\beta^{*}+\Delta_{\text {wais }}{ }^{2} / \beta^{*}$, asd $\eta_{x, y}$ to better than $1 \mathrm{~mm}$. $\mathbf{2}^{\mathrm{nd}}$ af aftects luminosity inditectly by perturbing the IP ango. ar spread, through the magnitude of $\left\langle\hat{\gamma}^{2}\right\rangle$ and $\left\langle\phi^{2}\right\rangle$, and hrough anomalous $\eta_{1,}$ and $\phi \phi$ coupling terms. Smalles spreat ñcreases $\beta^{*}$ linearly. Inversely, a larger sprend reduces it but Leo enhances the $3^{\text {rd }}$ order $\delta_{E}^{2}, \theta^{2} \delta_{E}$ and $\theta^{3}$ aberrations, w he criterion for optimal bslancing is no longer antisfied. The elative luminesity loss vermus $\beta^{*}$ is shown from aimulation in Fig. 4 for different $\sigma_{E}$. The shape is the same so in the chro matically uncorrected case in Fig. 2. Here, although the CCS has left $3^{\text {th }}$ aberratione, it hes removed the dominent $2^{\text {nd }}$ order terms, thereby raising $C^{\text {max }}$ and shiftin $\beta_{\text {opt }}^{\prime}$ towards amaller velues. The colerance on $\beta^{\circ}$ is about $\pm 20 \%$. Outside this range, the spot will not have the design aize even if the $1^{\text {or }}$ set of distortions are correcled. For $\beta^{*} 100$ lerge, $L \times 1 / \beta^{*}$ from

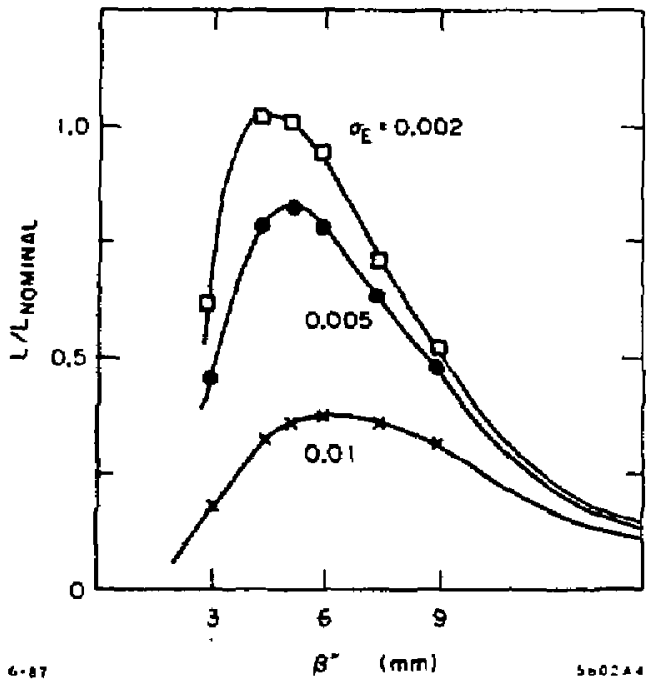

Fis. 4. Relative luminofity loen versus $\theta^{*}$ and $\sigma_{\text {. }}$ for a Final Focus chromatically corrected to $2^{\text {nid }}$ order.

linear optics. For $\beta^{*}$ too amall, $\mathcal{L}$ fullo off, firt linetyly, then parabolieally, as it becomes dominaled by $3^{\text {rt }}$ aberration.

\section{Optjeal Correctlon:}

The FFS could in principle be designed to fully match optical distortions generated upatream. Accumulated errors of any size would then be left for correction there. This it not feasible in the SLC because of the AJC, where minimizing aynchrotron radiation induced emittance growth requires a reasonable optical match throughoul. The $1^{\text {ot }}$ tep to apecify the matching solution is to set tolerance for the Arc and its input malch to evoid larze growth. Invertigatin such tolerances is beyond the scope of this puper. Let us uimply mention that the blow up is omalf for random imperfectione three times the specificd tolerance. ${ }^{7}$ Input mirmatch and systematic errors are more demagine.

The distortion's size then determine the range over which the FFS munt be tunable, and the above nenitivities how well one must match. Not all parametera are important. With no pinch effect initivlly and with of besically eet by the Linac, we consider distortions only in the four belatron dimensions and in their couplings to enercy. Betatron apuce is described by the voual o-matrix, with ten cerms $\theta_{j j}=\left\langle x_{i} x_{j}\right\rangle$. The $\boldsymbol{P}$-matrix deacribing the lattice has 16 terms. Output phase space in related to inpul by $\sigma_{\text {out }}=R \sigma_{\text {in }} R^{t}$ where $R^{t}$ is the tranopose of $\boldsymbol{R}$. For lines optice and neglecting aynchrotron radiation. Poincarre invariance requires, 10 that $\boldsymbol{F}$ be symplectic:

$$
R^{\prime} S R=S \text {, with } S=\left(\begin{array}{cccc}
0 & -1 & 0 & 0 \\
1 & 0 & 0 & 0 \\
0 & 0 & 0 & -1 \\
0 & 0 & 1 & 0
\end{array}\right)
$$

thereby retrictint the number of free terms in $R$ to ten. Applying the sume algebra lo the o-matrix, we find that the betatron space ear only be perturbed by the optics in aix independent weys. With the four dispersions $\eta t, 0, y, t$, we thus have a total of ten independent distorliont to correct. 
We choose to represent them by those for which tolerances were given above: the five IP angular sizes $\left\langle\theta^{2}\right\rangle,\left\langle\phi^{2}\right\rangle$, $\langle\theta \phi\rangle$ and $\eta_{1,0}$, and the five correlations of IP positions to angles and energy $<x\rangle,<y \phi>,<y \theta>$ (or $<x \phi>$ ) and $\eta_{x, y}$. Ideally correction would be done upetream of the CCS, to avoid perturbing it optimiratian relative to the Final $D_{\text {- }}$ magnifier. This is possible lar $\eta$, but only partially ponsible for betatron space.

Dispertion is correcled perturbing the g-match with fous quads, intualled in pairs $\pi / 2$ and $\pi$ phase shift from the IP, to control spatial and angular $\eta$, respectively. Each pair conaists of an erect and a akew quad for control in both planea. Natutally orthogonal for small input $\eta_{r, t}$ (the two erect onea perturbing the match of the borizontal lattice disperaion, and the two skew ones coupline it into vertical), they are coupled if $\mathrm{it}$ is large. Correction range is limited by quad strengths but also by the particular orientation of in put $n$. Some specific values make them ineffective. This happens, for example, when

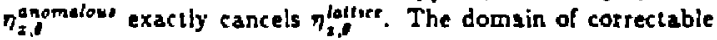

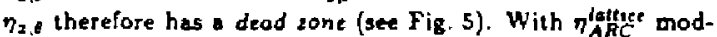
ulating around 35 cam with maximum slopes of $\pm 18 \mathrm{mrad}$, uncorrectable cases appear if, for instance, the horizontal betatron phase in the back of the Arc is off by a quarter of modulation cycle with the appropriate sign. Gross control is thus required upstream to bring 7 within eapture range.

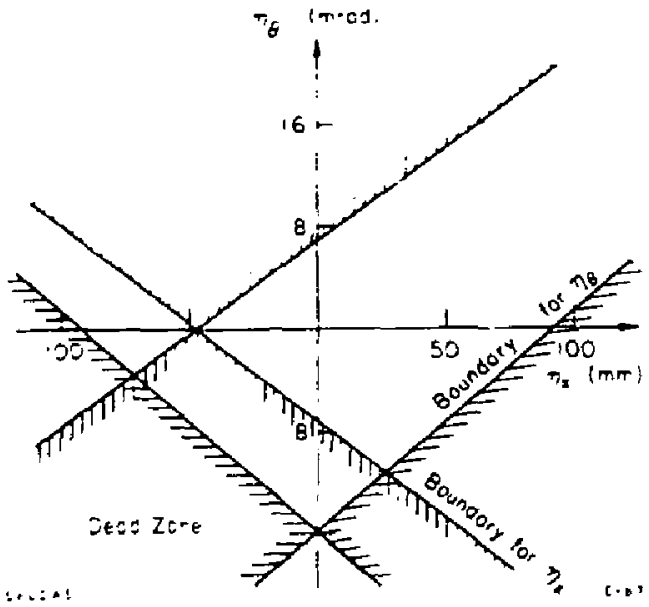

Fig. 5. Domain of correctable horizontal dispersion.

Betatron matching is oplit cross the CCS, in the B.match and Final Demagnifier. The three angulat terms $\left\langle\theta^{2}\right\rangle,\left\langle\phi^{2}\right\rangle$ and $\langle\phi\rangle$ are adjusted upstream of the CCS with two of the $\beta$-match quads and 2 skew quad, allowing to control the ratio of betatron to chromatic sizes in the sextupoles. The three waist offsets $\langle x\rangle,\langle y \phi\rangle$ and $\langle y \theta\rangle$ cannot be edjusted within the $\beta$-match independently of the angular terms. The are taken out at the very end, with iwo of the final lenses and a $2^{\text {nd }}$ kew quad. This atrategy does not maintain txactly the relative optimization of the Final Demagnifier and CCS, as the lat lenses are twesked. The luminosity, somewhat derated as nome $2^{\text {ad }}$ order aberration reappears, can be restored readjusting the sextupoles. Except for some cases of lasce errors, this in amall effect. With present hardware, the correction range is aboul $\pm 10 \times \beta^{*}$ for waist control (or $30 \mathrm{~cm}$ for $\beta^{\circ}=075 \mathrm{~cm}$ ) and a fector 3 in either direction for angular spread. Simulations and calculations ohow that random errors three times those expected in the Are can be handled.

\section{Overvlew of Tuning Strategy}

Turning 12 coupled knobs guided only by the IP spot-let alone optimining two colliding besms-would be very difficult without an ordered prosedure Since measuring $\mu$ m-sire beams is not straightforward, we must minimize the number of tuning experiments requiring IP infornation by diagnosing as many aspects of phase epace as possible before demagnification or wherever conventional instrumen ts are adequate.

Our tuning otrategy begins with optical matching of a single bean using strip-line Beam Position Monitors (BPMs) with about $20 \mu \mathrm{m}$ resolution and phosphor ecteens with about $35 \mu \mathrm{m}$ resolution. At the IP, crude measurements are done using thin carbon wires, about $5 \mathrm{\mu m}$ in diametet, from which spot bizes and centroids are inferred by bcanging. Such wire targets and BPMs neat the IP have been designed fot initial commissioning, but are also part of the early delector configuiation.

After both beams are minimiced, they are broughi and kegt in collision, first with BPMS near the IP, and then exploiting the electromagnetic fields from the bunches, which fot small enough size and large enough population cause them to be deflected if they miss each other " The ability to detect this effect delermines the size and intensity la be reached in the single beam phase. We shall see that about $5 \mu \mathrm{m}$ and a few $10^{10}$ particles is adequate.

Finally we maximize luminosity looking at beam-beam elfects in three ways: magnitude of deflection, synchrotron radiation from the collisions (Beamstrahlung) ${ }^{12}$ and distuption imaged in the extraction-lines. " Delector background'4 minimizalion is not covered here.

\section{Single Beam Spot Migimisation}

We first correct anomalous in put $\eta$. BPMs at the end of the Arc and in the FFS are used to measure bearn motion versus energy. This does not give the position-energy correlation within the bunch if anomalous $n$ exists where the energy is varjed, but gives a good estimate if the Arc is (as expected) the dominant contributor. As more accuracy is needed, one must constrain $\eta$ in the Linac or measure spot sizes directly. With $1 \%$ energy scans, we determine $\eta$ to a few $\mathrm{mm}$, or about $5 \%$ of the Arc average. Using a model, we determine $\eta_{1, d y, 6}^{\text {angmalo }}$ from a least-square $5:$ to the measurements and csleulate the matching solution. Locally orthogonal "knobs" are derived for fine-tweaking. We first correct the $\beta$-match, to enable measuring betatron phase space there. Final settings for IP corsection will differ if the $\mathrm{CCS}$ and Fina| Demagnifer generate $\eta$, for example, through orbit errors. The sesu/ting n/P is mostly spatial as the lenses there are $\pi / 2$ phase shift from the IP. Final IP correction is inferred from BPA1 measurements and from measuring $v^{\circ}$ versus the derived orthogonal knobs, using the wire targets.

Betatron phase space is then diagnosed measuring spo: size on acreen versus the strength of an upstream quad, and fitting a parabola.'s Special emphasis is put on emittance, to help guide tuning in the Arc. Since c enters as a overall scale, we must resolve the parabola's minimum. For a quad and a screen separated by $I$, this requires $d^{2}, \beta>r^{2}$, where $r$ is the scjeen resolution. This condition is met beloce demagnification, in the $\beta$-match. A setup with about $100 \mu \mathrm{m}$ minima in both planes is installed. Twiss parameters, found from the parabola's axis and branches, serve to diagnose gross betatron mismatch. $x$ $y$ coupling obscuring the s-measurement is diagnosed looking al the tilt of the spol versus quad strength, giving another parabola and three mors terms. With nine terms in total, we can in principle fully determine betatron space. In practice, being mostly interested in . while brtatron mismatch is better 
easured elsewhere, we simply correct for the measured $x-y$ supling.

Betatron mismatch is best diagnowed in the Final Demacifier, where angular and spativ sizes are naturally eparated. sing the three $\beta$-mulch knobs, we frat at the three anguler त्wis $\left\langle\phi^{2}\right\rangle,\left\langle\phi^{2}\right\rangle$ and $\langle\phi\rangle$, looking at a nominally sund spot on $a$ high-p ecteen upstrem of the Final Triplet. Je begin, first ignoring $t_{x, y}$, by wanding the beam upricht -ine the akew quad. Then, taking $c_{x, y}$ into account, we peritb the two sixc with the erect quids, so at to best antinfy ie fopt $\propto$ ct rcaling law (minimizine $3^{\text {rd }}$ order aberration). (ter this, the beam will have the predicted vive at the axivd sition where it comes to a waist, although that place may - offet from the IP. Correction, amountine to cencel $\langle x\rangle$, yt $>$ und $<x \phi>$ terms, is talculated sweeping the three

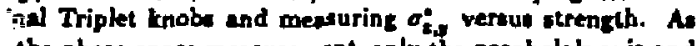
the phare-space menurement, only the parabola's axis and anches are needed. The axis give the offsets and the branches c derivatives of size versus atrength. In principle, resolving e minima is not essertial. With all lenses $\pi / 2$ from the IP, can be shown that the skew quad is fully orthogonal to the o other waist controls, provided the $\langle\phi\rangle\rangle$ coupling term properly nulled; however, the latter two are coupled. The -orithm first orthogondizes them using the derivative and en eets them based on the measured axis. The skew quad is justed equivalently before or after. MIP can loo be cancelled th tuch parabolic oweeps. Find minimization is schieved itting all the corrections.

\section{Steerine the Beama in Collinion}

The average mutual defiection of two gaussiun beams coling at an offset $\Delta$ inll

$$
\theta(\Delta)=\frac{-2 r_{\varepsilon} N_{T}}{\gamma} \frac{1-\exp \left(\Delta^{2} / 2 \sigma_{T}^{2}\right)}{\Delta} F\left(\sigma P / \sigma_{T}\right)
$$

ere $r_{e}$ is the classical electron radius, $\rightarrow$ the relativistic factor, the nuraber of particles in the target and $\sigma_{T, P}$ the turget 1 probe sizes. $P(r)=\frac{L\left(1+r^{2}\right)}{r^{2}}$ is a form factor computed for all $\Delta$ by folding in the probe diatribution. It reduces the rate for $\Delta=\sigma$, whereas it should be dropped for $\Delta>\sigma$, the beams then wee each other a poiat charges. Deflection sus ofiset is thown in Fis. 6 lor $50 \mathrm{GeV}$ beams with $5 \times 10^{10}$ ticles and 2,5 and $10 \mathrm{\mu m}$ izes. Detection is best done the system's high- $\beta$ points, near the Einal Triplet, where ranslates into the lartest possible thift. Special BPMs are ifoed ${ }^{16}$ for this purpoes.

The tuning method proceeds in three stepu:

1. Initiv bean foding: Atter bringing the beans close I BPM near the IP, one of them (the most intense) is led on and of while the other it measured at the outgoing h-p point. This tives the ohift induced by the collision, the in velting in which direction vo uteer. For hrge $\Delta$ and with cerical factort, we get $\delta X_{\text {out }}(\mu \mathrm{m}) \approx \frac{t 0 N_{r} / 5 \times 10^{10}}{\delta / 100 \mu m}$.

2. Beam centering: Scannint one beam across the other enoptimal centering on the tero-deflection bymmetry point = Pis. 6). The lareent shift occurs for $A \simeq 1.50 \mathrm{~g}$ giving

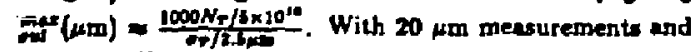
-ut $2 \times 10^{10}$ particles initially, we expect good signals for = 5 mm. This cets the goal for angle beam optimization.

3. Feedback: The mutual defiectiona are tro used to keep iwo beamp in collinion. In timple vernions of weh feedbeck, sample position devistions from an initial reference at rexipal high-p points an ingoing and outgoing paths. Drifs in the the approximated by $\Delta(t) \propto\left(6 X_{\text {ouf }}(t)-\delta X_{\text {in }}(t)\right)$. A slow

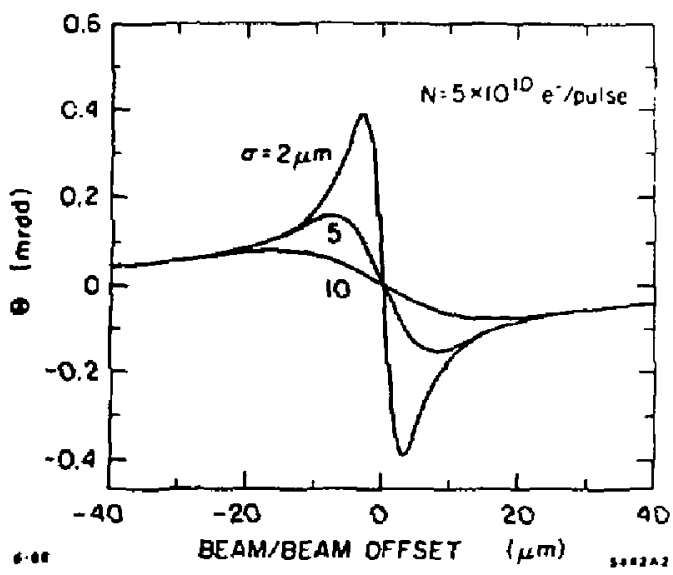

Fig. 6. Beam-beim deflection versus offet for three spot sizes.

version of such a syttem, with a time constant of about 20 seconds, is aviilable an an extension of existing software. ${ }^{27}$ More sophisticated pulse to pulse schemes with optimal filtering ${ }^{18}$ of specific frequencies are in probres.

Luminosity Optimiestion with Beam-Beam Efiect.

Algorithms based on three besm-beam signals are in prosress.

1. Magnitude of deflection: The maximum deflection pro duced in the centering scan is atrong function of beam sizes and can be used for tuning. For centered beams, this signa] allows separating improvements in the two planes, but unfortunately not in the two beams. This can be seen noticing that after normalizing by $N_{T, P}$, conservation of the total transverse mornentum implies equal average deflections for each bearn. The maxinum must thus be oymmetric in the two beam sizes, making it herd to know which one needs to be optimized (except by rensing derivatives).

2. Beamstrahlung: ${ }^{12}$ The total photon flux enitted in the collisions is atrong function of beam sizes. For each particle, $N_{2} \propto \sigma_{n} / \rho^{2}=\sigma^{2} / \sigma_{n}$, where $\sigma_{n}, 0$ and $\rho$ are the bunch-length, deflection angle and radius of curvature. For centered beams of equal aize $\sigma_{R}$ and populations $N_{1,2}$, Beam 1 radiates $N_{1}^{\text {lotal }} \propto$ $N_{1} N_{2}^{2} / 0_{r} \sigma_{R}^{2}$. Without the ahepe of the photon beams it is hard to separate the two planes. One can however distinguish the lefget from the amaller by ceanning one ecross the other. This is the reversed situation from the deflection gignal, making the two methods complementary. The resson is the $\theta^{2}$-dependence of $N_{r}$. leading to a num of aquares for $N_{\gamma}$ iotal. The dependence of $N_{7}^{\text {fotal }}$ from the probe versus $\Delta$ is indicated in Fig. 7 for a target with equal, larger or amaller tize.

3. Diaruption: When beams have been made small and intense enough, they act s lenses for each other, thereby increating their angular apread der collision. In the linear approximation, of grows by about $50 \%$ for besms with $2 \times 10^{30}$ particle and $2 \mathrm{~mm}$ tranverne cizen. Monitoring of this effect will be possible in the extrection lines, by imaging IP anales on acreens through optics designed for the planned energy spectrometers. 13 Tuning for the fugeat possible spot on these acreens will then meximize luminosity. 


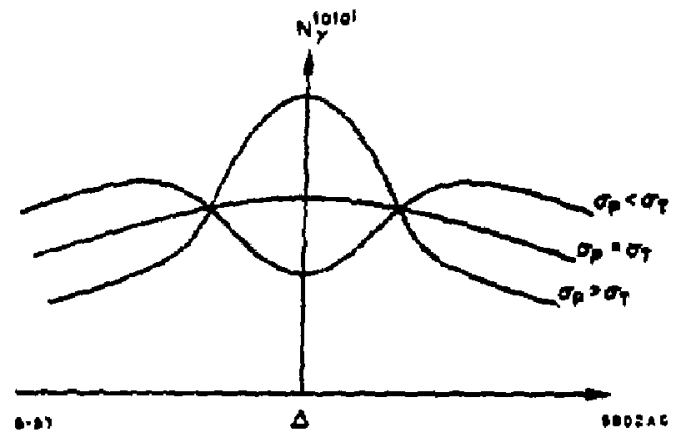

Fis. T. Tota/ beamstrahlung tex vereus oficet for unequel kronoverue beem tsen.

\section{Acknowledramants}

I aro indebted to neveral SLC and Mart II colinesue for fruitful diecussion. Specisl credkt ehculd be glven to K. Brown, T. Fieguth and J. Murrey for designins the optics, to W. Kontnecki and R. Erickson for leadine our group, and D. Riteon for reviewing many of our aleorithons.

\section{Beferences}

1. SLC Design Handbook (December 1984).

2. J. J. Mursay ef al, The Completed Deaign of the SLC Final Focw Syotem, these Procendinge.

3. K. L. Brow'n, privale cammunieation (June 1987).
4. J. J. Murtay and T, Fiesuth, MURTLE, Privale Procram.

\$. K. L. Brown el al, GIAT Commitlee Report (February 1956).

b. R. Y. Saruranckx, private commugication (Jure 1987).

7. S. Khellat ei C., Beam Optical Denion and Studies of the SLO Ares, BLAC-PUB-1013 (June 1DBe).

จ. K. L. Brown of al., 8LAC-01, Rev. 2 (May 1977).

D. E. D. Courant at al. Theory of the Altemaling-Gradient Synchrotron, Ann. of Phym. 3, 2-48 (1058).

10. L. C. Tent, Conearbire A-Dimerrional Coupled Motion, Fermilab Interael Report FN-320 (MSay 2071).

11. P. 8. Bumbede at el., Beam-Bean Dofection of on Jnics. action Point Diopnastic Jor the SLC, SLAC-PUB-3070 (May toto).

12. G. Bonvieint at d., Beamulpaklung Monitor for SLC Final Focue Voing Gammo Ray Enerpies, SLAC-PUE3900 (Muy 2006).

13. Mark II Coll. and BLC Sind Focu Group, EatractionLine Spetrometere for SLC Eneroy Mearurements, GLAC-SLC-PROP(3) (3nst).

14. W. Komancel3, GIAT Committe Report (Junuary 1086).

15. M. C. Row et al, Bigh Revalution Beam Profile Monitora in the SLC, SLAC-PUB-3040 (April 1988).

16. J. C. Denerd of al., Moniloring the Bcam Ponition in the SLC dinteration Region, then Proceeding.

17. K. A. Thompeon et al., Fredhack Sustems in the SLC, theare Procendinge.

18. A. Btienint, Sampied Peesdact in the Linear Collider, SLAC CN-14 (November IOSO). 
VII.3 "First Order Optical Matching in ${ }^{4}$ : $:$ Pinal Focus Section of the SLAC Linear Collider"

This publication describes the modeling and fitting algorithms that were developed to perform on-li... model-driven optical corrections. The tuning algorithm is summarized, and some initial experimental results presented. 
SLAC - PUB - 4021

CALT - 68 - IAOB

Mey 1980

(A)
FIRST ORDER OPTICAI MATCHING IN THE FINAL

\section{C.M. Hawkes}

Califormia Inditule of Technolopy, Peudena, Odifornia olres

and

\section{P.S. BAMADE}

Sesnord Linur Acealerutor Center, Stanjard Univervity, Stanfond, Californie afsoo

\section{ABSTRACT}

The procedure uned for dinperion and betatron matching in the lint order opticel taning of the SLC Final Foew ure dacribed. Anociated modelligy and Giting urorithou are presented.

A straightorwerd anthod for artending untoupled optieal models to incorpotale croneplane coupling is axplained.

\section{Submitsed to Nucjart Entruments ard Metbod A}

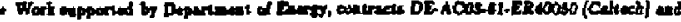 DEAvas-7aspoonts (BLAC)}

\section{INTRODUCTION}

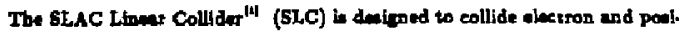

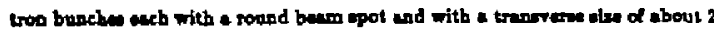

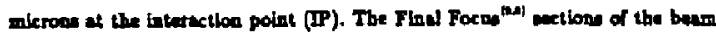

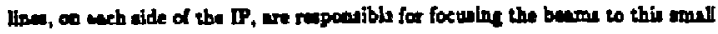
ine.

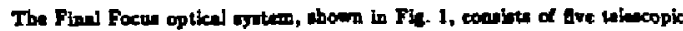
module. Demagnifiention is achjerwd in tro etages, in the Fint and Find Telowcopes. The chrometleity of the quidrupols arity lo compenested throuth wextupelea is the Chromalk Correction Section. Matehiag of the dispention and the betatron motion to the Are hation is provided in the r-Matebing Section and the Fint Trimcope repectivaly. Extraction of the opent beam, moving in the oppotila diraction, it achieved in the Fint Talemeope, through a pulaed mapmet and a mptam.

The Find Focw munt wo provide reriable optical mutehing for the correctoe of dintortions in the latties wring through pradient ersors in the buan lise upatsanem nud within the Fiad Focu Bection itealf. Thit note decriber two parts of the optical turint procedure ued doring the comminitoning and running of the SLC Final Focke: the dispenion and betalron matehing.

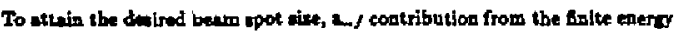

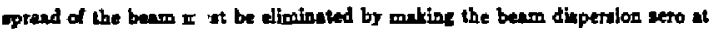
The IP. The method for doing thie in decribed in Section 2.

The BLC denien be equel trithen if the borisontal and vertical plenes. In

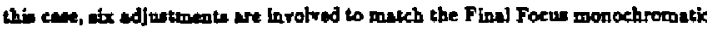

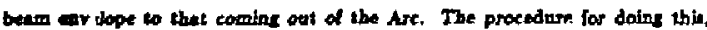

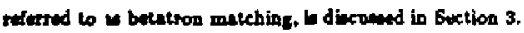

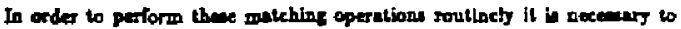
heve detalfed optien modellind and fietine included as pert of the online cantrol profram for the Find Forw. The exintine onliwe SLC optikal model was not edequate tinte it anume that the band dyambles to the borisontal and vertical phane are independeal and bence cannot aceommodste crow-plass coupline. A atraluthtorward end zeraral mathod for exterding the model wo incorporate eromplane coupling wa deriend and to explained in Appendix A.

\section{DISPERSION CORRECTION}

\subsection{DETputrons}

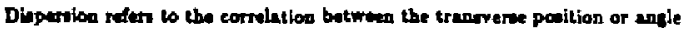
of a particle b a bunch and it energy relativg wo the mean onergy of the bunch; 6. gerinentel opatial diapersion:

$$
\eta_{n}=\frac{\Delta r}{b}
$$

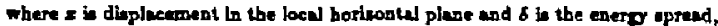
$\delta=\Delta E / E$. Mort precisely thl can be kermed the boum dippereion", It corrapand to the atrtb column of the ben oremetrix" and contributen to the

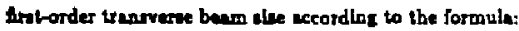

$$
\sigma=\sqrt{c t+\left(b_{T}\right)^{2}}
$$

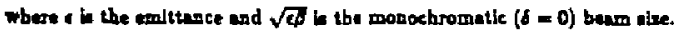

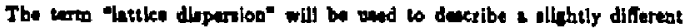
quantlty. It is a property of the optical lattice of the beam line, indopendent of the barm, und corsempond to the dirib column of the trander matrix, of $R$ -

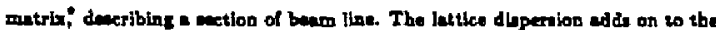

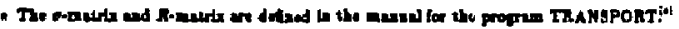

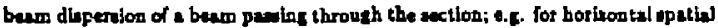
dispention from point A wo point $B$ :

$$
\eta_{i}^{E}=R_{13} \eta_{\hat{A}}^{A}+R_{12} \eta_{i}^{\prime A}+R_{13} \eta_{\hat{r}}^{A}+R_{14} \eta_{p}^{\prime A}+R_{16}
$$

where $\eta^{A}$ Is the baum diopetsion at the otart of the wetion, $\eta^{\mathrm{D}}$ in the beam dupenion at the and $\alpha$ to rection and $R$ is the transfer matrix acrows the ection. The horizontal opatial lattice dispenion from $h$ to $B$ is $R_{10}$.

\subsection{Destan Dtspersion}

The SLC require: ero opacial and anguiar beam disperion (n ond $\eta^{\prime \prime}$ in * and $y$ at the interection point in order to optimine the fuminosity. With on ener ag aprend of $0.5 \%$ a reidual uptial bearg dispersion of only $1 \mathrm{~mm}$ will more thas double the spot nixe from 2 wo 5 micron.

Fis. 2 abown the dajeg nut for $\eta_{x}$ as a funetion of disterce along the beam

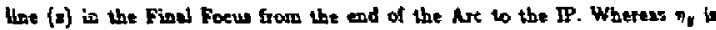
deriened to be exo everyabe a the Final Focis, this in not true of $\eta_{x}$. The condition $\eta \mathbb{P}=0$ in kehieved by having non-kero beem dispension at the end of the Arc, $\eta^{A R C}=17.5 \mathrm{~mm}$. This is cancelled by an equel and opposite lattice dimpentan in the n-Matching Section at the bezinnlng of the Final Focu, leading to $\eta^{P T}=0$ at the end of the Fint Teleacope (FT). The Chromatic Correction Section (CCS) temporavily seberelee large value of $\eta_{*}$, wert of its function, but the desiza value of $\eta_{\mathrm{x}}$ returne to wero $\mathrm{at}$ the end of the CCS and through the Final Telececope to the IP. 


\subsection{A MOMALOUS DEPERSION}

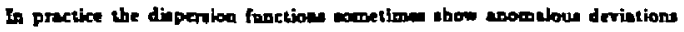

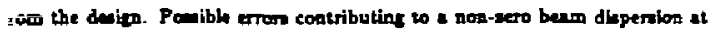
LIP can be erouped into two clenes.

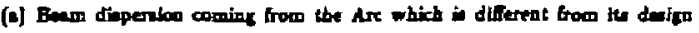
alue.

(b) Anomblous diepersion ariniss trom erron in alements of the Find Focw beam line inelf. These are moat likely to come fram the CCS (due to mimaligment of a extupole of quedrupole, for exemple\} tince $\eta_{\text {in }}$ is locally

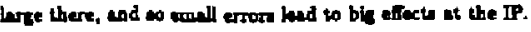

It in deireble to deal with arrorn of type (a) fint by correcting the dinpersior

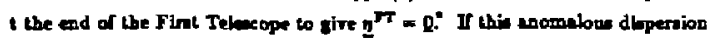
Lef: uncorreted at the and of the FT it will propactive throush to the IP. The

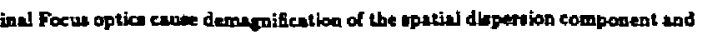

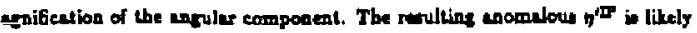
2 be of mucb greater magitude thes $\eta^{F}$, making it dificult is masure $\eta^{T}$ -curately eoough for it wo be corrected at the $\mathbf{P}$.

Erron of type (b), combined with rmidual errore of type (a), must then be indled eperately by correcting the dippeniod at the IP to clive $\underline{n}^{\mathrm{IP}}=\mathbf{Q}$.

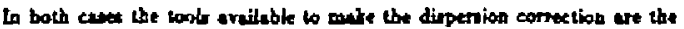

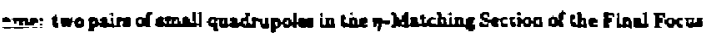
-ith ooe apicht and one atew quedropote in each paly. Thene are abowo crow-

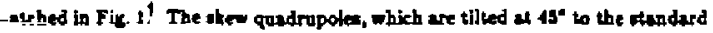

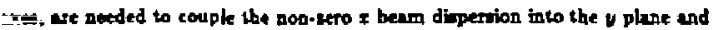

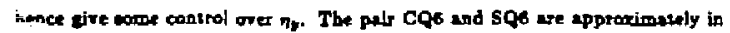

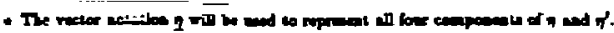

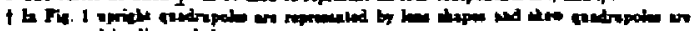

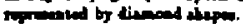

phen with the eod of the FT wbetwe the pair CQS and 595 are approximuleb

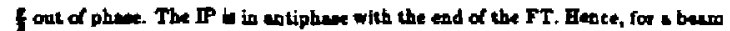

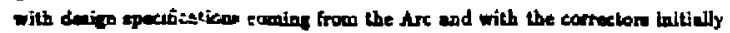

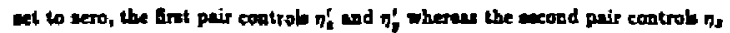

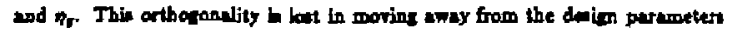
for the beam and latice. Bowerer it in alragr ponible to find a combination of four quadrupole etting to correct the four $\eta$ components at the end of the FT or at the IP. The oaly limitation in the meximum eurrent arvilable from the mugnet pomer euppliea.

\subsection{MEASURTNG Ditersion}

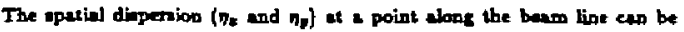

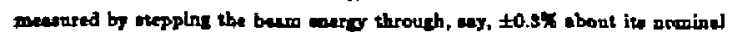

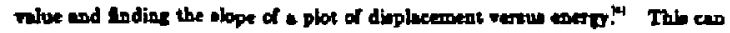

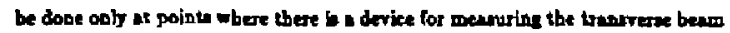

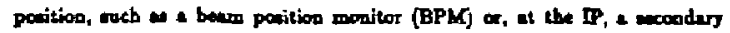

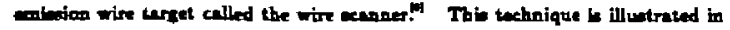
Fit. 3.

To fifer the opelial and angoler diapartion at any polat \& fit must be made

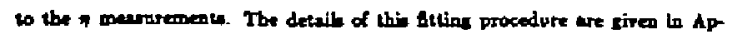
pendix $B$.

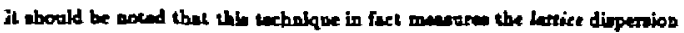

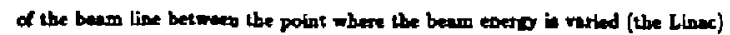

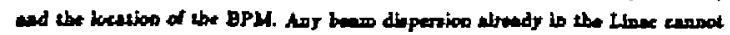

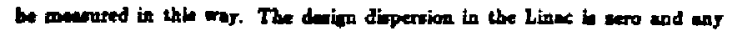

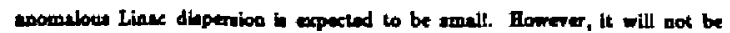

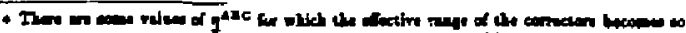

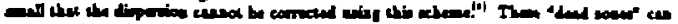

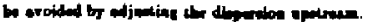

mearured and bence will nos be corrected by the Firs: Jeleacope DLin-rsinCorrection cheme to be decribed in Section 2.5. In will propecke through to the IP and must be corrected there an deacribed in Section $\mathbf{2 . 6}$.

\subsection{F IRSt TElescope DLPERSION CORRECTION}

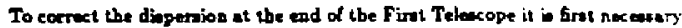
os determine the opaiv and anguler depersios at the end of the Arc. pare. Thl in done by menruring the optial disperaion al BPMe nes the end of the $A r c$, a decribed is gectien 2.4, and then urint the bitind technique deacribed In Appesdix $\mathbf{B}$.

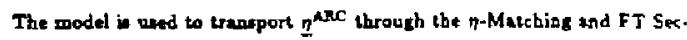
tinne of the Final Focus to give $\underline{q}^{\mathrm{TT}}$ at the end of the FT:

$$
\underline{\eta}^{r T}=R^{A R C} \rightarrow r T\left(k_{1}, k_{1}, k_{3}, k_{1}\right) \underline{\eta}^{A R C}
$$

Dhere, now,

$$
\begin{aligned}
& \underline{\eta}^{A N C}=\left(\eta_{*}^{A R C}, \eta_{n}^{\prime N R C}, \eta_{k}^{A R C}, \eta_{j}^{A A R C}, 0,1\right)
\end{aligned}
$$

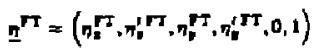

and pevart in ebe trander onertix from the ead of the Arc to the end of the FT ealenlated from the matel. The sixth elewent of the $\underline{\eta}$ rectorn, which is dwas pnity, envure that the inlive diperion elements contained in the find column of $\boldsymbol{R}$ ure comectly incorporated ecrording to equation (2.3)

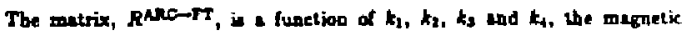
otrengthe of the four dippenion corrector audrapoles deacribed ebove. In order

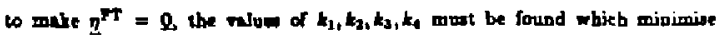

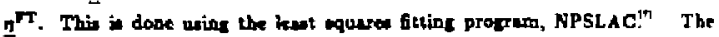

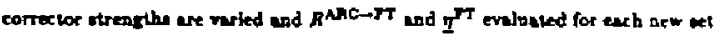
of olrengthe. The miniminstion program exits when the oun

$$
\sum_{i=1}^{4}\left\{n_{1} T\right\}^{2}
$$

b rnfieiently imall. The corremponding vilues of $k_{1}, k_{t}, k_{3}, k_{1}$ we returned and can be uned to est the atrength of the phyaied magnets.

Thin algorithm wan und resulurly durine the last period of the SLC Final Focw commimioning ind and by now become routine and biqhly reliable. Correction und ly yield a dipertion fonction within a few per cent of the deagn. One exmple id ahowa in Fis. 4, Up to now, the ultimnite limit to the prexision of thi correction ba ben omell disnmeat errose on the dispersion corsection quadrupole themelve. A their atrencthe are raried to perform the earretion, the minalignment caune tbe bear to be ateered a well a focued downtrean and bence the reult in not cuacly o prodieted by the online modiel.

\section{Imteraction PONT Deperston Corregtion}

Dren after a anceneful $\mathbf{p}^{\mathbf{T}}$ correction there might atill be beam disperion at the IP which mont be corracked. This can be due to roidual anomalous diperion propactating from the FT ar due to alignment or mapotic error in the Final Focu beam line linelf.

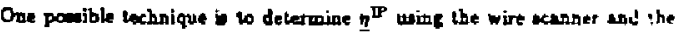
BPML par to the IP and the measurement and fitting scheonen deacribed in Sec. tion 2.4 appendix B. Corrector retting en then be cakulated which will perserate an equal and opponite $-\underline{q}^{P}$. On iteratios the IP dispersion should terd coward sero. There are two dicudvantente to thi wethod. Fincly it repuire en eceurate menturement of $\eta^{\mathbb{P}}$, A explained in Section 2.1 thin in dificult becaure of the apetial demegnification produced by the Final Focun. Secondly.

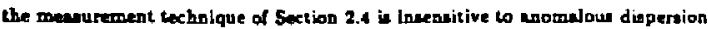


generaled is the Linec of further uputrend, nince it ectuslly menures the lallice diepersion between the point where the beam energ in varitd and the measurement device. Bence thi contribution to the beer dimperrion et the IP wauld no: be contecled.

Another uxhnique involve minimining the true beas disperrion at the $I P$ by

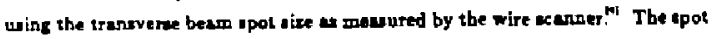

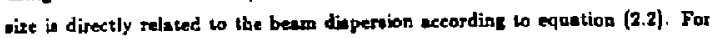

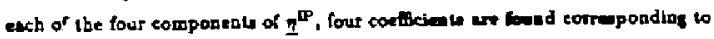
the changes in each of the lour corretor quidrupole aeting aesded to produce - unit charce in that component of $\underline{q}^{I P}$ while leuving the otber three cadpo nents of $\underline{\eta}^{\text {IP }}$ unchuged. Thene reprent orthogond "n-tnobs" tor controllins the dipernion st the IP: A wasen thes be mede over a suituble ranife of ench component of $\eta^{\text {TP }}$ and the rite of the apol at the IP menured at each point

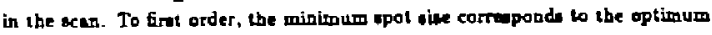
carrextor eettinge

\section{BETATRON MATCHING}

\subsection{Betatron Phase-Space DestoRtions}

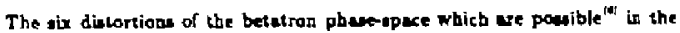

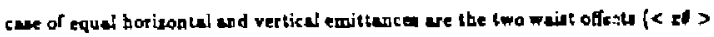

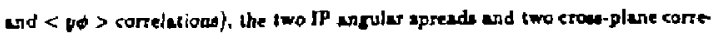
bations of IP angles $(<t \phi>)$ and of IP position 10 ancles $(<x \phi>)$.

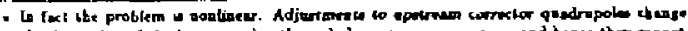

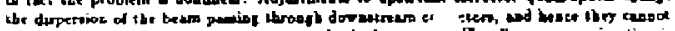

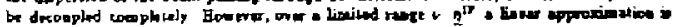
valud

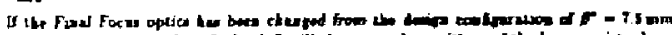

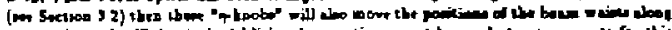

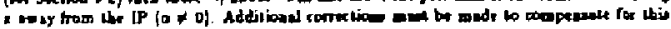
thex
}

The wigter spread terms are controled upotems of the CCS bJ cancellint the < o > croan werm with a akew quadrupole (SQ17.5) and by norine $\sigma^{\circ}$ in enc

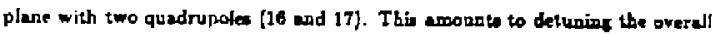
demagnification factor in the Find Focu beam line. Waint werme are corrected at the very end, with trim winding on the let quadrupoles (2B end 3) and another akew quadrupole (SQ3) immedievely aptream. Two additiosed kerm (< IV > and < $w$ ) would require ed ditional comection element in the ene of anequal erritunces, but are reduadant otbernive. All six quedrupote whicb enot be controlled to whieve thin are shaded is Fig. 1 .

The wints must be positioned at the collinion point to Fitbis the depth of feld $s^{*}=1 \mathrm{~cm}$ and the $\langle x \phi\rangle$ correlation remored

After cancellint the $\left\langle\phi \phi>\right.$ crow-term, $\sigma^{*}$, wbich for fxed emithence deler-

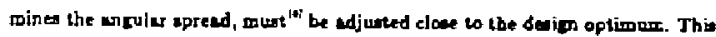
optimum $\propto$ sure when linear optice and hithes order efect contribule bbout

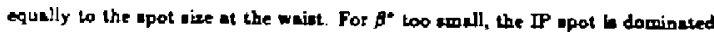
by chromatic aberrations (third order if the uscond order chromatic correction ta

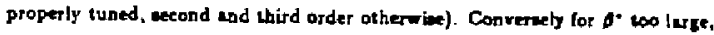

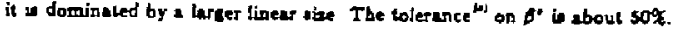

\section{CoRrection ProcedLRE}

Waist controls can be made orthogona, lines and is dependent of input phase-pect distortion, allowins position to ande corrolation in the IP opor w be conculled br empirieally minimining lu wite. Thi i dode wing the wire ecanner." Spote are measured a function of the ortbogond wabl contrals, and

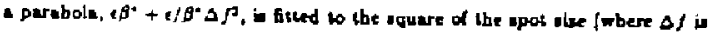
the distape. I he wiaty lo determine both a and $\theta^{4}$,

Actual engular cpreadi are well delermined from the branebes of the parabole.

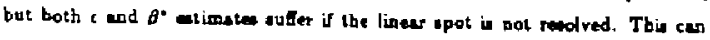
ure through residual crou-plare coupling, only parially corrected in cave of ugequal emittences, and through third order chrometic sberration which can dominuk before the betatron ma'ching if fully implemenled. Sextupoles murt we be fitued in the perturbed lattic at each iteration of the betsitron malching procedure decribed bere, 10 praserve goo second order cortection.

Mensured value of $\beta^{*}$ are und to determine enpolut corrections needed for matchine. The edjuat ments are ponlioes functions of the three quedrupoles unad for control. In eddition, the corrector ale afrect the wains. The woluton muat therefore be the reulf of a overall ft of the aix correctors toward the devired IP phan-dpace. The etrins proeedure is outlined in Section 3.3. Because of the

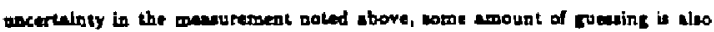
tecusery wo deurmine the mouhing correction required. The proctdurt coul in ceneral be itesated in an empitical cearch for the optiond entriar correction.

The online modelline and attine packnge developed allows full texibility in

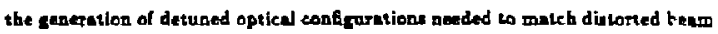
ervelopen, band an mensured of on atimeled optical parameters. The fittins procedure cen aleo be adjusted convenienrly by uter intervention, to wil opecitic situations. Thi is explained is mare daceil in Appendix C. An anople of auch deluned colution is ahow is Fic. 2. The wlid $A_{1}$ and $A_{1}$ currew whow the deaign letice of $\rho^{*}=7.4 \mathrm{~mm}$. The denh-doted eurve ahow a detured confororation

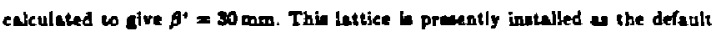

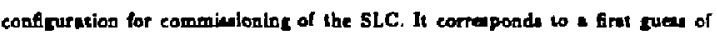
the most probuble angular correction needed wo metch panibly varying optical

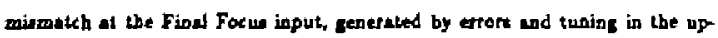
otream aratems. In this configuration, residual anguler errora are small enough to reproduce amall If apote a the five micras fevel by weepins only the three wint control. It it anticipaled that additional iteratione of this melchine will be required to optimive the apot tive furthet.

\subsection{TJTDG Procedunt}

Before aterting the lat atage of betatron matcbing it in anomed that, to a fret epproximation, the disperion ha been corrected a decribed in sexLion 2, and the whint and ero-plene coupling corretion bive been compleke a outlined in Section 3.2. Bence $a_{k 1} a_{\text {, and }}$ al the croat-plase terms in the beson $a$-molsix" at the IP we approximately sero. The named initial a-matrix ("oth ineratios) in:

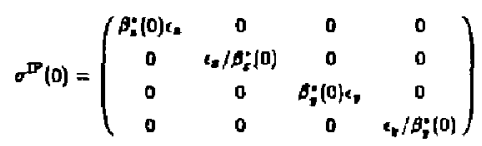

It is distonal and can be apecified by fout indepeodent parameker. Any two out

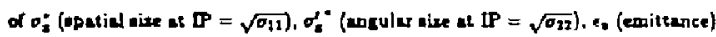

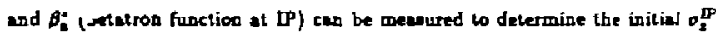
metrix, and similarly for $y$.

The o moerix of the beal the end of the Arc it then

$$
\sigma^{A R C}=R^{-1}(\underline{k}(0)) \cdot \sigma^{I P}(0) \cdot\left[R^{-1}(\underline{k}(0))\right]^{-T}
$$

whet $R(k(0))$ is the initial transfer matrix from the thd of the Arc wo the IP and

$$
k(0)=k_{1}(0), k_{1}(0) \ldots, k_{-}(0)
$$

we the initial etrengthe of the a quadrupoles and okew quadrupoles in the Finat Foew beem line which are uned for the betotron matching

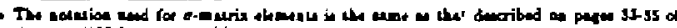

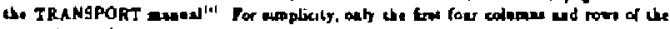
o.eneriar are dhoes.
} 


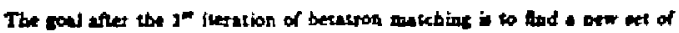
wotheth, $\Delta(1)$, wuch thet

$$
\begin{aligned}
& \sigma^{J}(1)=R(\underline{k}(1)] \cdot \alpha^{N A C} \cdot[R(\underline{k}(1))]^{T} \\
& =\left(\begin{array}{cccc}
\theta_{*}^{*}(1) \epsilon_{2} & 0 & 0 & 0 \\
0 & c_{0} / \theta_{i}^{*}(1) & 0 & 0 \\
0 & 0 & \theta_{i}(1) \epsilon_{0} & 0 \\
0 & 0 & 0 & \sigma_{y} / \theta_{0}^{*}(1)
\end{array}\right)
\end{aligned}
$$

- the devired o-mutrix of the IP. To so fram atution (3.1) 10 (3.4) the opatjel

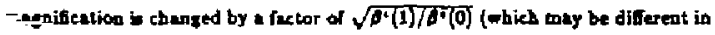

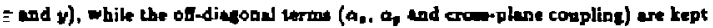
It sero. Tbe detait of the 6tting alforithm und ere prenented in Appendix $C$. The quodrupole ttrength, $k(1)$, sturaed by the stear after the first iteration zen be wed to wet the phyaicu menets on the beam line.

The beam opot it thea remensured ind furber iterations of the matchins joucedurt are implemented to optimive the Inoninosity.

One example of a watl spot mesured during the 1977 SLC commianionint b whow in Fic. 3. At the time of writing the amalet electron pot aise which heve so far been obtained were menured at $3-4$ micron. Simoltaneous eleciron and panitran upot cizes of 5-7 microns ean be reproduced without dificuley. Commingioning wort is contibuing to brins the SLC parteretert up to the leve! itcater for the production of $2^{\circ}$ erenta.

\section{APPENDLX A}

\section{MODELLING CROSS-PLANE COUPLING}

\section{A 1 TWLS PARAMETERS}

In the SLC costrol protreom the atendard colise optical model of the

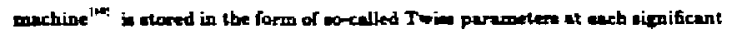
poinl along the beam line:

$\psi_{2}=$ the botiontal bethuton phew of tune (cometimed denoted by $v_{n}$ )

$\boldsymbol{A}_{\mathbf{x}}=$ the borivontal betatron fuection

$\alpha_{*}=$ the boriwontal apha fanction

$7 x=$ the borizontel eparial dippertion

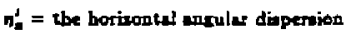

and timituly in the rertical (y) direction. The Twin parmeten are genereted by ronsing the peopas COMFORT (Venion 3), wins a inpol either deaign

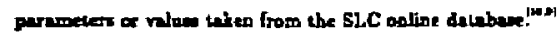

Is order to model a enction of the beam line betwetn poirts $A$ and $B$ it in

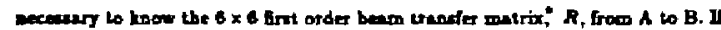
the aptics in $x$ and $y$ plesen are independent then it enn be writus in the form

$$
R=\left(\begin{array}{cccccc}
R_{11} & R_{19} & 0 & 0 & 0 & R_{14} \\
R_{21} & R_{11} & 0 & 0 & 0 & R_{30} \\
0 & 0 & R_{41} & R_{41} & 0 & R_{40} \\
0 & 0 & R_{41} & R_{41} & 0 & R_{44} \\
0 & 0 & 0 & 0 & 1 & 0 \\
0 & 0 & 0 & 0 & 0 & 1
\end{array}\right)
$$

with all cron-plane couplins alemente al sero.

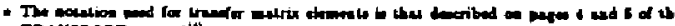
IRANBPORT ane'

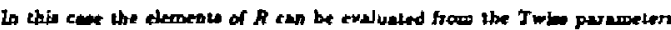
at pointe $A$ and $B$ waine aranderd formulac. For $x$ :

$$
\begin{aligned}
& A_{11}=\sqrt{B_{B} / \theta_{A}}\left(\cos \Delta \psi+a_{A} \sin \Delta \psi\right) \\
& R_{13}=\sqrt{A_{A} A_{2}} \text { ain } \Delta+ \\
& R_{16}=\sigma_{B}-R_{11 T_{4}}-R_{11 n_{\alpha}^{\prime}} \\
& R_{\text {y }}=-\left\{\left(1+\alpha_{A} \alpha_{B}\right) \operatorname{din} \Delta \psi+\left(\alpha_{B}-\alpha_{A}\right) \cos \Delta \psi\right\} / \sqrt{B_{A} \beta_{B}} \\
& R_{n}=\sqrt{\rho_{\mu} / \beta_{\theta}}\left(\cos \Delta \phi-\alpha_{0} \sin \Delta \psi\right)
\end{aligned}
$$

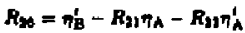$$
\text { where } \Delta \phi=\phi_{\phi}
$$

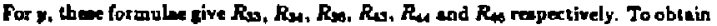
the trander matrix elemente only the change in the $T$ wim parameters between points $A$ and $B$ is aeeded. Their abeolute value at acb point aze not important for this purpose.

\section{A2 Cross-Plakt Couplakg}

The procedure outlined in Section A1 i valjo wij if there is no couplin: betreen $x$ and $y$ plene rince the $x$ and $y$ Twien parameter are aused to be todependent. That in, it work only if the tranefite matrix can be witten in the form of equation (AI).

Thi i valid reprementution for mant atenderd beam line elements, but it 1. invelid for aket quadrupole and rotations of the coordionle nyelem (rolls) Gince alew quadrupole pley a vital part in the SLC Find Foew optics it was necentery to modity the ondine ordelling.

One colution would be to abusdon the preant model and raplace it with one - bere the full two-dionemand trancfer matrix to ach bean llow point is otored.

Since COMFORT (Veriog 3$)^{(1)}$ dow not include eromplane coopling this would alo aeed to be uppradedt

Inatead it was decided to ketp the Twine puramater and to add a relatively umall amount of extre dediceted Finel Fecus bodelling and bttins code lo handie crom-plane coupling where this was necenary. To do this the model bewn line is divided into a erie of diverete, equential antions of two worts:

(a) thowe for which cros-plepe eospling in unimporengt, i.e. Tbart art no pkew quedropole, roll or wimile besm line elemeats;

(b) thow for which crom-plene covplas is iropothent.

For entegon (c) the oaline Twien parameter for the points the bezinning and end of the ention are aufieient. The foll twe-dimengiond tranler matrix ran be found uing equation (A2) and (A1).

For calefory (b) the Twien perameters eanoot be wed. Bowterer, tbee sec-

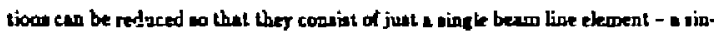

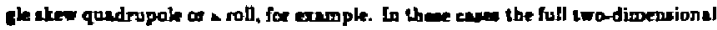
trander matrix ean be determined expertely uing a watl gumber of is pot parameter laten from the onlipe dalabave. In the case of a akes quadropole the dits required are the incepaled magetic feld grdient (otrengh), the eliective length of the mugat, the akew angle and the evergy and churge of the beam.

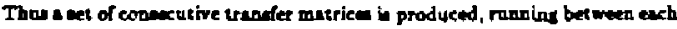
of the polate slong the whale beam line. By coultiplying thl otring of matrice together in the correct wequince the cumulative trander matrix between any two of the polist ean be obtained. This in all that in needed fot a model inçorporatint eros-plane coupling.

If is eometime ueful to enlere caktory (b) above. For exumple, wo perform

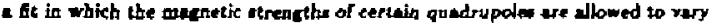

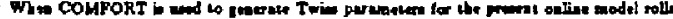

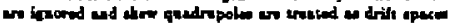




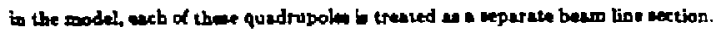

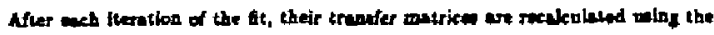

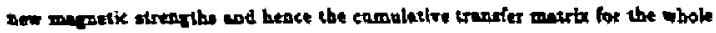

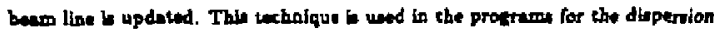
and bentroa matchitag whieh are decerbed la the andp text.

\section{APPENDIX B}

\section{DETEAMRATION OF SPATLAL AND ANGULAR DIRPEPSION}

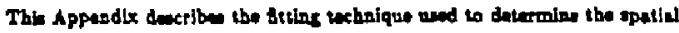

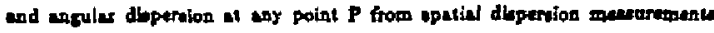
mude by beste poaltion monitors.

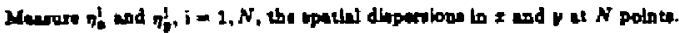

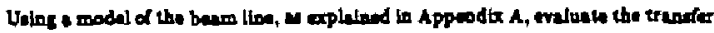

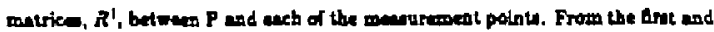

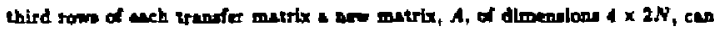

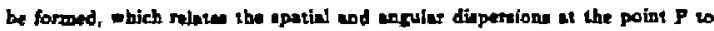
the epatid dipercina menuremeptu:-

$$
\Delta \underline{\underline{\eta}}=A \underline{\underline{q}}^{\mathrm{P}}
$$

where

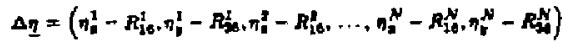

$$
\begin{aligned}
& \underline{n}^{p}=\left(n_{1}^{p}, \eta_{*}^{p}+n_{p}^{p}, \eta_{z}^{p}\right)
\end{aligned}
$$

und the rom of $A$ we formed from the rowe of $R^{\prime}$ by $(j=1,4)$ :

$$
\begin{aligned}
& A_{1,}=R_{21}^{1} \\
& A_{x}=R_{5}^{1} \\
& A_{\mathrm{d}}=R_{\mathrm{j}}^{\mathrm{a}} \\
& \text {; } \\
& A_{(t N-2) h}=R_{11}^{N} \\
& A_{2 X_{1}}=\text { P }^{N}
\end{aligned}
$$

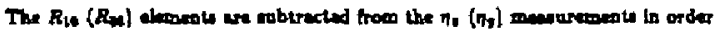

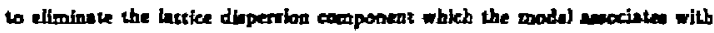
the beaw lise between $P$ and the menurement pointe. The remainder, $\Delta \underline{n}$, to

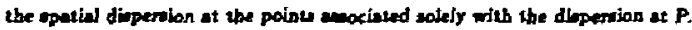

In ordes wo colve for $\eta^{p}$ the matrix aquation (BI) muet be inverted. Since $A$

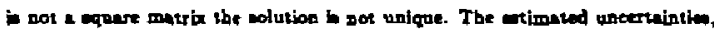

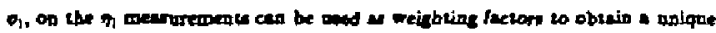
invertion The $x^{3}$ wum:-

$$
\sum_{i=1}^{N N}\left\{\frac{\left(\sum_{j=1}^{+1} A_{j} \eta_{j}^{P}\right)-\Delta \eta_{i}}{\sigma_{i}}\right\}
$$

b minimined by tating

$$
\underline{\underline{Y}}=(B A)^{-1} B \Delta \underline{\underline{Z}}
$$

where the elmesels of the $2 N \times 4$ dimentiond matrix $B$ are foresed from thane of $A$ by

$$
B_{\mathfrak{g l}}=\frac{A_{y}}{\sigma_{1}^{j}}
$$

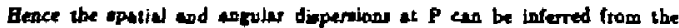
menuratents.

\section{APPENDIX C}

\section{FITTING ALGORITEM FOR BETATRON MATCEDNG}

The delatl of the etring algorithon uned for the betutron mulching acheme decribed in Stetion 3.3 are prenented in this Appendix.

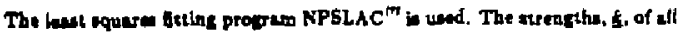
the quadrupola of be aned for the betatron metehing ere revled end $R(k)$, the

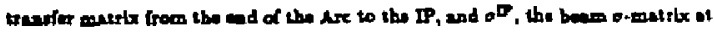

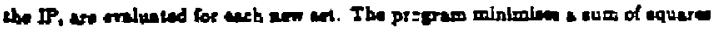
compond trom the deroente of $\sigma^{\circ}$ :

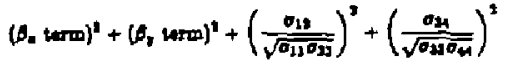

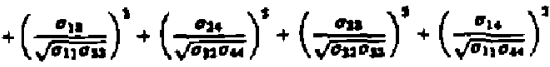

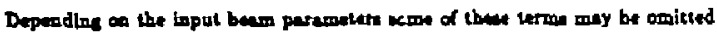

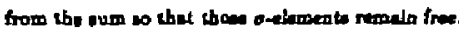

The third and fourth ketmin of thip mus contrio a, and a, wo ramain at sero

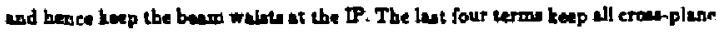

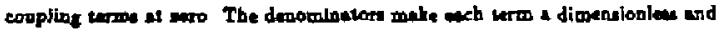
rasonably aceld number.

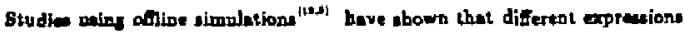

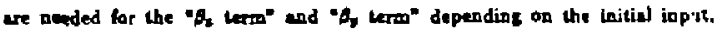
to order wo moure that the fit cooverese rapidly od reliebly. Fot many ispu. condition, wille:

$$
\theta_{n} \operatorname{term}=\frac{\sigma_{22}-c_{2} / \theta_{g}(1)}{\omega_{2} / D_{1}(1)}
$$

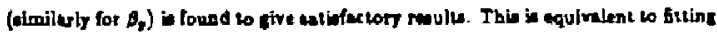

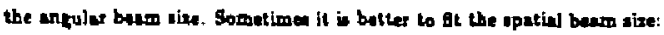

$$
A_{x} \text { term }=\frac{a_{11}-p_{k}(1) c_{x}}{\beta_{k}(1)_{1}}
$$

of comatimen upatial and angular oben together.

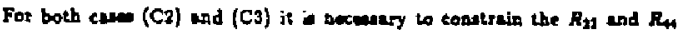

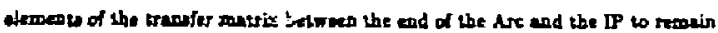

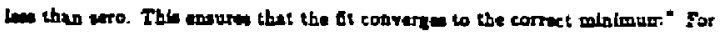

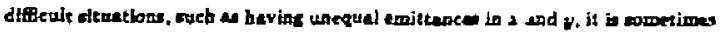
better to Bi thene eleoreate diretly:

$$
B_{.} \text {Lerto }=\frac{R_{22}-R_{29}(\mathrm{fit})}{R_{20}(\mathrm{ft})}
$$

- were $A_{p o}\left(f_{1}\right)=R_{m}(t(0)) \sqrt{D_{*}(0) / B_{F}[1]}$.

The eficiency and reilience of the fitine prograx can dwo be improved by

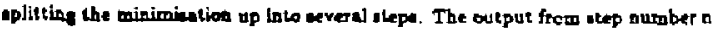

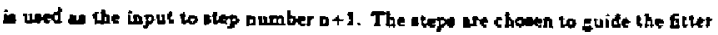

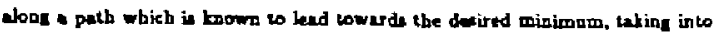
ccoust the propertio of the optice of the Find Focum bestr line. For e range of input conditiana the following two-step $6 t$ in found wo buitable:

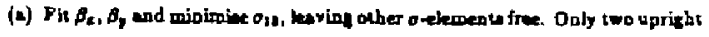
quadrupole (17 and 10] and the acarby ske qaedrupole [5917.5) are

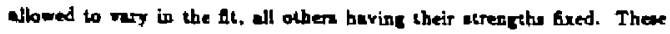
quadrupales we in the Fint Telmeope apd are the onea moet aens.'ive to $A_{2}, D_{1}$ asd $o_{13}$. Quidnipole 17 and 16 control the overall demarnification

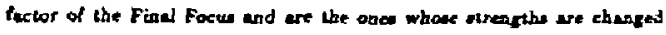
thos dufing the betatron matchina.

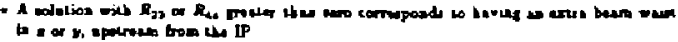


(b) Minimise $\alpha_{0}, a_{\text {, }}$ and $\sigma_{4}$ while keeping $\beta_{n}, A_{4}$ and $\sigma_{3}$ at the ralues they have ther olep (a). In eddition to the three quedrupole varied during atep (a), two more uprlaht quadrapole (3 and 2B) and the nearby okew quadrupole (Bg3) wo sho allowed to vry. There are in the Floul Telecepe. Shep (b) enounte to a minot ret aning of the optica to reposition the borisantal and

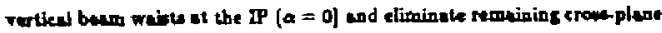
eooplins.

Thi tmonep st is the defanit weed in the SLC coetrol program, bot frimt Iexibility in avilable for changing the Guting alporithm when becesary.

\section{ACKNOWLEDGEMENTS}

We with to thest all the people who bere belped in the dewign and comminaloning of the Find Focel and the rest of the SLC.

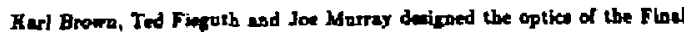
Focus. Ted, Joe and Dave Biron contribuled in weveral major ways wo the taning atorithon and correction procedure thet were eratved and that we now wed.

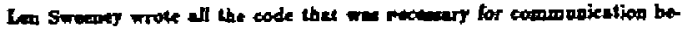
tween the modelling od fitline proporm decribed is this peper and the reot of the SLC control noter. Be slo produced the control panele and dbploy.

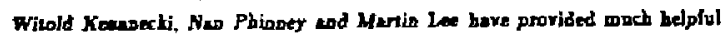
adrice.

We aro thant Mercarel Wright and Philip Gill of the Syotem Optiminatiop Laborntory of Staviord Univenity for cratting a modibed wention (NPSLAC) of their NPSOL"n 6tting pockete for wo in SLC optical modelling

\section{REFERENCES}

1. SLC Desipr. Bandbook, SLAC (Dexember 1984)

2. Moger A. Drickon: Find Focu Syrtem for Liqen Colliders, U.S. Sum-

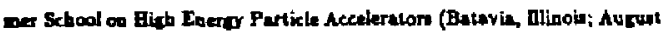
1904). SLAC-PUE-1470 (Norember 1907)

J.J. Murn, K.L. Brom, T. Fiegutb: The Completed Deipo of the SLC Fiol Foes 5rmeor, Parlicle Acoslerator Cooference (Weahingtop, DC: Mereb 1007). SLAC-PUR-1219 (Febrany 10a?)

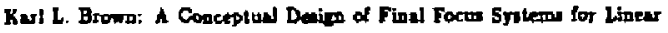
Callider, Joint US-CERN School on Particle Acculeratont (Sootb Pudre. Tean; Octobe 1006). SLAC-FUB-4150 (Jone 1967)

3. P.S. Bambade: Bam Dyamica in the SLC Final Foed Syatem, Particle

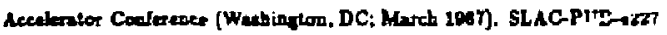
(Jupe 1987)

4. K.L. Browa, F. Bobseker, D.C. Carey, Ch. belin: TRANGPORT - A Cowputer Propram for Delening Charted Particle Beam Transport Sylems. SIAC-OI, Rev. 2 (May ieT)

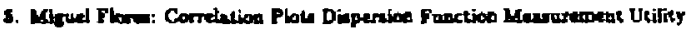
(February 10a7), anpubliched

6. C. Field et d: A Bigh Reolution Wive Seanter for Mierod-Sive Proale

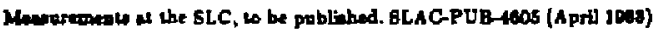

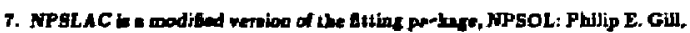
Welie Karray, Michel A. Sennders, Marcaret B. Wilght: Uner b Goide for NPSOL (Vertion 4.0): A Portran Pecluse for Nonilinear Procramming,

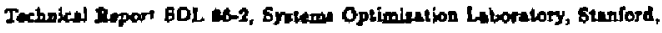
CA 94305 (J. 7 2046)

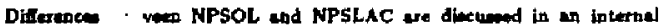

alAC Single Pus Collider Memo: Chrio Hawkes, Martis Lex: Recent lpetadine of tha Modelline Protram COMFORT, CN-342 (September 1986)

1. W. Kocenechi: Propres Report on the SLAC Lnew Colfides, Levernetional

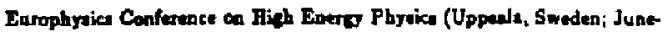
Jub 1007). SLAC-PUB-445 (Nowarober 1907)

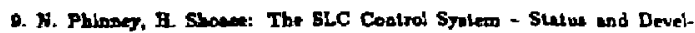

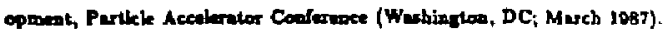
BLAC-PUB-1215 [Marcl 10T)

N. Phmary: Report on the SLC Control Spleo, IEEE Trans. Nucl. Sci.,

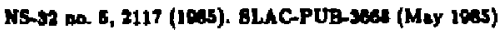

R. Y. Molen: Deripg end Performance of the Sturford Linear Collider Con. trol 5 men, IEEE Trase Niel. Sci., NS-32 Do. 1, 230 (1045). SLAC-PUBS467 (Oetober 1944)

10. M.D. Woodhy, L. Sanebe-Chopiten, E. Stowe: Application of Online Modeling to the Oparation of SLC. Particle Accelaralor Conference (Wash-

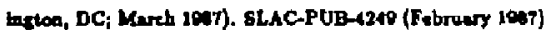

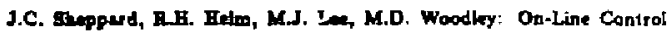
Madele for the Sturford Libear Collider, IEEE Trans. Nucl. Sci., NS-30

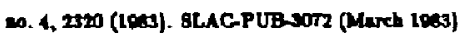

11. L. Bunchat, B. Showe, M. Woodky: COMFORT - Control of Muchine Proction, Drbita, ad Trajetorim, Veribo 3.0, SLAC (Martb 1906), uspabilinad

M.D. Woodky, M.J. Le, J. Jiser, A.S. Kint: Control of Machide Functions or Treneport \$yatems, TEEE Trans. Nuel. Sel, NS-30 ne. C. 2367 (1983). SLAC-PUB-3006 (Mnreh 1043)

Bes alo Ref. 10.

12. W. Kanmetil: private communicalion

\section{FIGURE CAPTIONS}

1. The optleal deisp of the SLC Final Focu. The fout quedrupolen uned for

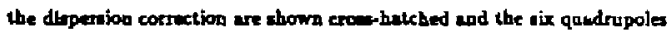
and for betatron matching are ahown aheded.

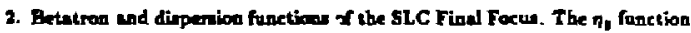

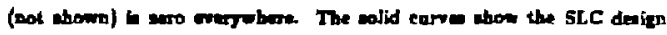
wherean the denh-doticed carve ahow $A_{2}$ end $A_{\text {, }}$ in the del uned $\beta^{*}=30 \mathrm{~mm}$ conopontion deribed in Baction 3.2 of the muis taxt.

3. Dirpertion Menrurement Technique. To pearore toe latlice dispertion of a

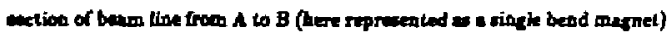
the bear ard $b$ inerwad from $E$ w $E+\Delta E$ and the beam definction at point $B_{1} \Delta x, i$ merrund aning a BPM. The dispersion in $\Delta x /(\Delta E / E)$.

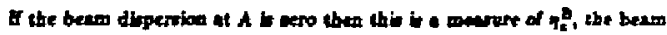

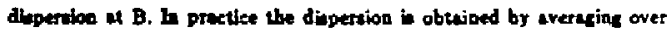

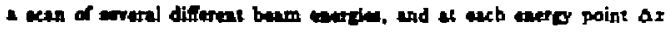

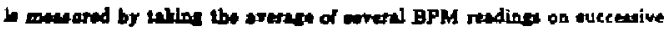
beam poines. 
4. The Firet Tolecope Dlepention Corretion. The horlwoated (upper Bture)

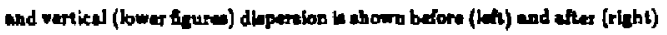

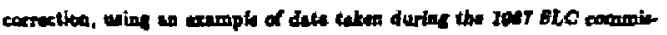

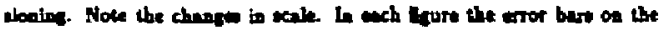

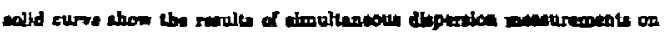

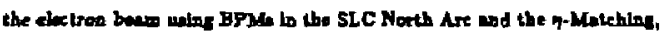
Fint Telecepe and Chromalic Corretion Section of the Nerth Final Fo-

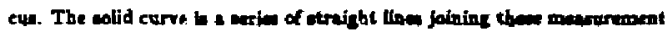

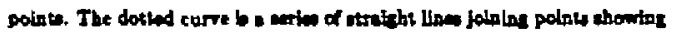

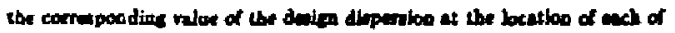

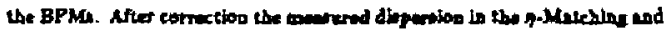
Fint Talecope Sectlone in very elase to the deniph reluen.

5. As anople of a tranvere cen of the olectroe bem the inverecton

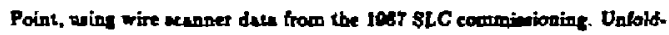

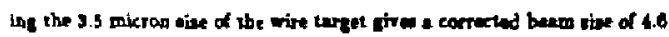
ticrom.
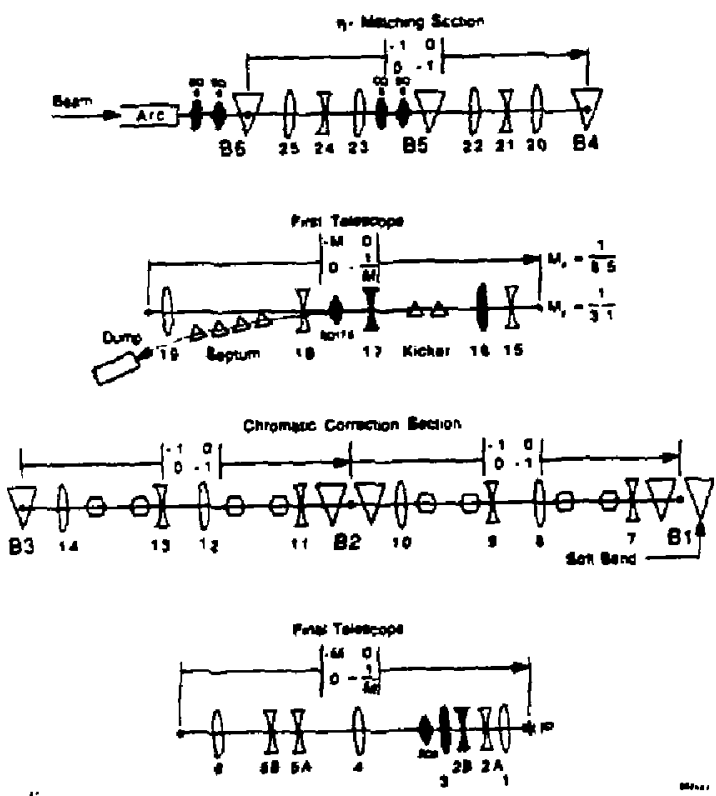

fip 1

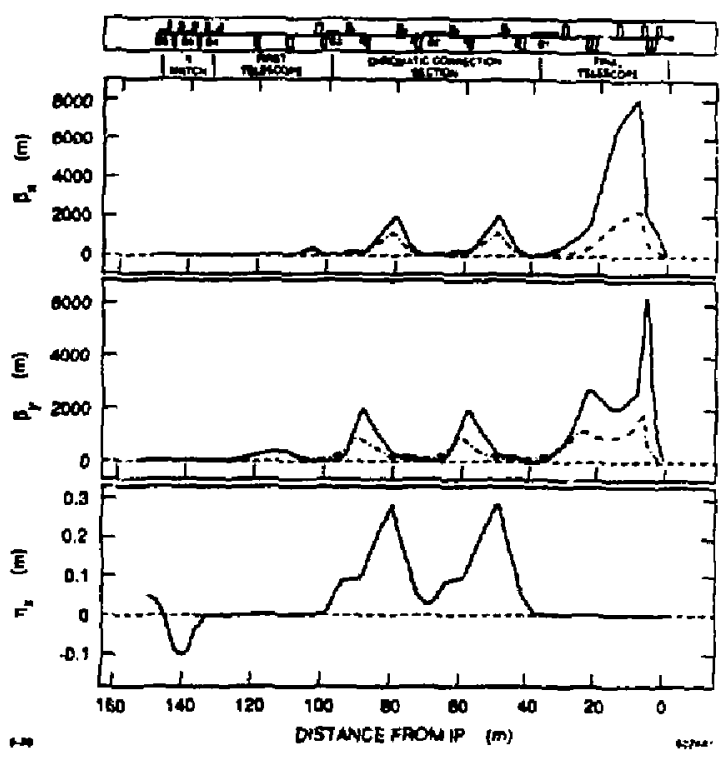

Fig 2

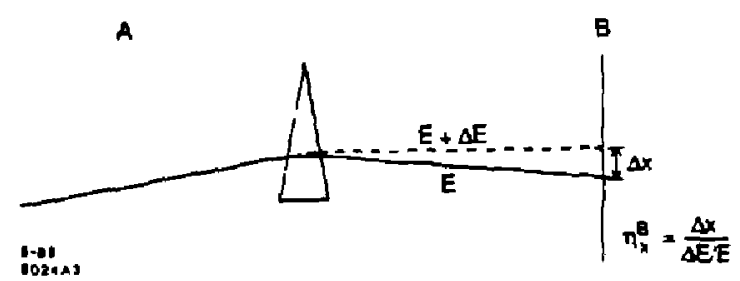

Fig. 3 

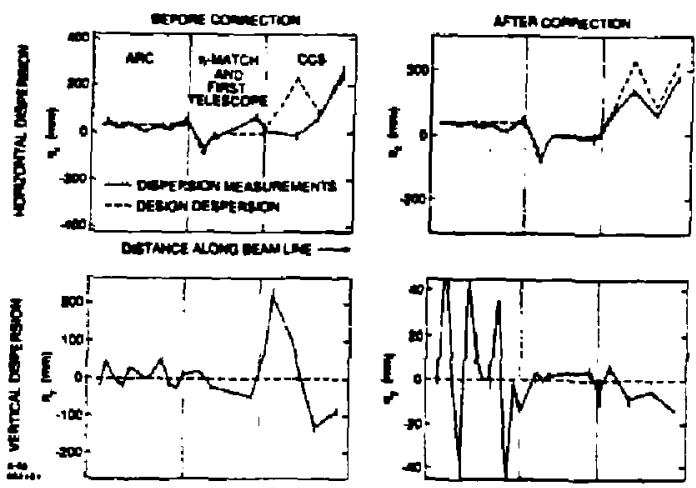

Fig. 4

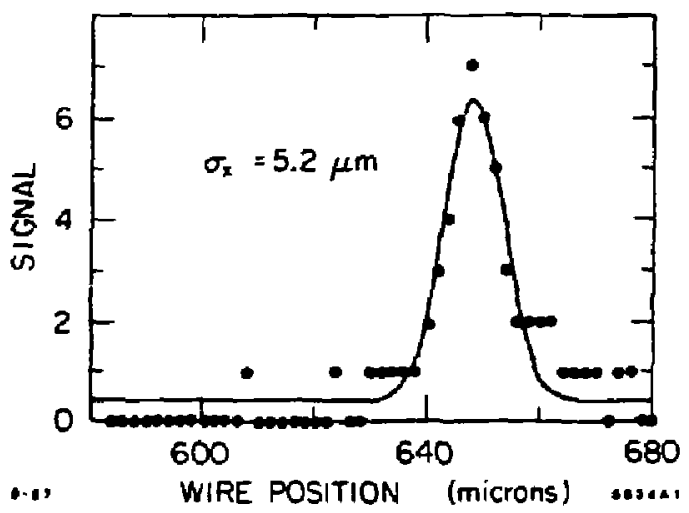

Fip 5 
VII.4 "Operational Experience with Optical Matching in the SLC Final Focus System"

This conference article summarizes the experience acquired with the optical tuning algorithm developed for the final focus. The algorithm and some of the control software are reviewed, and a comprehensive set of measurements are presented. The algorithm has allowed to focus spots at the collision point with transverse sizes of 3 to $5 \mu \mathrm{m}$, and to diagnose its various components. The residual beam size at the collision point is limited by a somewhat larger than nominal emittance delivered by the linear accelerator, and by the requirement to minimize backgrounds in the detector, from which a larger than nominal $\beta^{*}$ parameter must be used. 
SL. If PI $11, \ldots$.

Jitn:lat:, 10 ,

+ 1

\title{
OPERATIONAL EXPERIENCE WITH OPTICAL MATCHING IN THE SLC FINAL FOCUS SYSTE.M*
}

\author{
P. BaMbade; P. BUMChat, D. BURKE, W. Ford, C. HaWkes, W. KOSkA.' \\ W. Kozanecki. T. Lohse, T. Mattison, N. Phinney, M. Placidi, D. Rutson, S. Wagneg ${ }^{\prime}$ \\ Stanford Linear Accelerator Center, Stanford, Univeraity, Stanford, C.A 9,309
}

\begin{abstract}
In the SLC Final Focus System, all componetsts of transrerse phase-space and the couplings betreen them must be controlled to minimize the beam size at the interaction point. After summarizing the experimental algoritim and the on-line tuning prograns, we present a consiatent set of messurements and describe our present understanding of the various contributions to this bean size.
\end{abstract}

\section{INTRODUCTION}

The Find Focus System (FFS) is the last section of bearnline in the Stanford Linear Collider (SLC) before the interaction point (IP). Its main function is to maximize the luminotity by focusing the beam to the smalleat possible size. Because the beam bis a finice enictance and energy spread, a nominal beam size of $2 \mu \mathrm{m}$ at the IP can only be achieved with elaborate optict where higher order abertations are curefully minimized. ${ }^{2}$ In operation the FFS must also be tunable to absorb focuting errors accumulated in the transport line upatrenm and in the FFS itself. ${ }^{3}$ Effects from such errors manifest themedve primarily as linear mismatches between the tranjerac phasespace of the injected beam and the FFS optica, and must be oorrected before the final focusing works praperly. An experimestal tuning agorithm bas been developed ${ }^{3}$ to achieve then wroctions, and extensive operational experience bas been ecquired."

Initially it was thought chat this tuning would be used as an overall correction for mismatchea accumalated in the eatire SLC. or at least in the Ares; except for of few opecial canes, it is possible in principle to absorb optical diatortions of up to a facLor of four. ${ }^{3}$ It was also thought that variations would be tuned continuously in the FFS. Neither appess to be fenojble. Elimination of backgrounds ${ }^{5}$ in the detector from electromagnetic debris and muons produced when bearn-tails strike aperture limils upstrenm of the matching elements requires a nearly mutched phase- ipace at it.jection. Thus, major mimonatches must be corrected upstream, and in practice only small adjustments are made in the FFS. The min limitation to continuou optical feedback are lack of orthogonality in the corrections and the faet that the only place avalable to diagnone all the dintortions is the bigher order conrected foca] point at the IP. As a result of noportbogonality, eves modest varistions is the incorning phase spece cap require extensive retuning. These weaknease reault

- Work supported by the Department of Energy. contracts DE-AC03-76SF005 I5, DE-ACO2-76EROI112, DEAC02-86ER40253. DE-ACO3-81ER40050 and DE-AM03$76 S F 00010$. from adding-on the optical and background tuning strategies to a design where the besic architecture was already fixed. and suffered from severe space limilations.

Operationally, the optical tuning has evolved towards determining a stable set-up. Party because the Linac emiltance presently exceeds the design value by a factor of about three in the horizontal plane," the optics must be configured with a larger than optimal $\beta$-function at the IP $\left(\beta^{*}\right)$, in order 10 reduce backgrounds generated in the last quadrupoles by the beam tails. The larger $\beta^{*}$ and Linac emittance limit the attainable luminosity. Phese-space parameters are monitored routinely to distinguish stable changes from spurious ones. and to base corrections on time-averaged quantities. After reviewing the optics, the tuning strategy and the on-line programs used. we describe measurements made with tbe electron beam in the last nun (September 1988). Similar results have been obtained with the positron bearn.

\section{SUMMARY OF OPTICS}

The FFS consists of four telescopic modules (Fig- 1). Opti. eal demagnification is achieved in the first and final telescopes. which otruddle a chromatic correction section where the intrinsic first-onder chromaticity of the beam-line is compensated. The Arc lattice $\eta$ and $\beta$-functions are matched in the $\eta$-match section and in the firat telescope, respectively.

The optimization of the chromatic correction is the central point of the design. ${ }^{2}$ The Chromatic Correction Secticn (CCSI consists of two - I telescopen, combiaed with dipoles at the foci. to generate signifieant energy dispersion at the quadrupales. Sextupoles, where the focusing strength varies linearly with ex. cursion, are put aear the quadrupoles to provide additional focusing proportional to energy. This dlows cancellation of the intrinic first-order chromaticity. Additional first-order perturbutions to the imaging produced by each sextupole are made to carcel over the length of the CCS by eppropriate symmetries. In this way, all renidual perturbations are pushed to second-order. The efective $\beta^{*}$ cas thus be written:

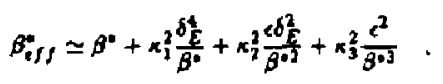

where $x_{1,3,3}$ messure tbe megnitude of the residual second-order chromatic and geometric perturbations, $c$ is the emittance. and $\delta_{E}$ is the fractional eper $\boldsymbol{C} y$ apread.

The effect of the chromatic correction is to branden the en. ergy band-pass aver which rays are imeged to the sime IP loral point. The width of this band-pass scales roughty as $\sqrt{5}$ if

\footnotetext{
- Calitornla institute or Techmoloriy. Pasadena. Ca 9lizb

Universitr or Michigan, ANN AMLOR. MI 18109

- CERN. CH-1211. Geneva 23. Switzerland
}

Bat 200, Oanat. France 91405

I Limiveasitr or Califonnia Santa Couz. Ca gsoba

i Univensitr of Colomado. Bothper, CO 80300 

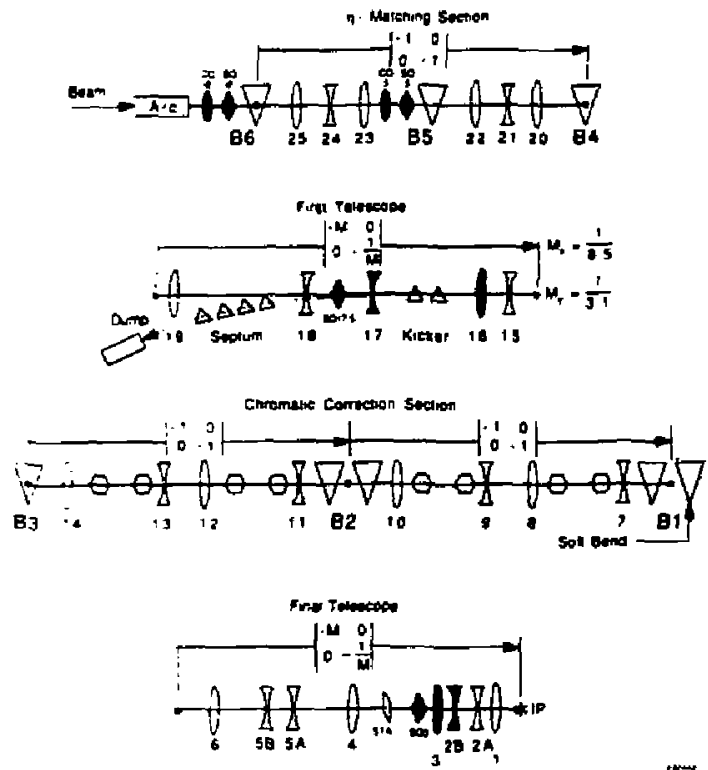

Fug. 1. Sehemalic of the Find Focus. The four quadrupoles used for dispersion cornections ane shown cross-hatched, and the siz quadrupoles used for betatron corrections ane shown shaded.

only the term in $x_{1}$ from (1) is used). ${ }^{2}$ Defining it quantitatively as the band of energy deviations for which $\beta^{\circ} J \leq 1.25 \beta^{\circ}$, it is $\pm 0.5 \%$ for $\beta^{*}=16 \mathrm{~mm}$, and $\pm 0.22 \%$ for $\beta^{*}=4 \mathrm{~mm}$. Without chromatic correction, it is lese than $\pm 0.05 \%$ in both cases (Fig. 2). emittances from correcting only these len distortions, is close to $4 r /(1+r)^{2}$, where $r$ is the ratio of the smaller to the larger emitunce. The reduction can become severe for $r<1 / 4$.

The distortions are best characterized by what matters physically at the IP:

1. Five correlations of positions to angles and energa: $\sigma_{21}, \sigma_{43}, \sigma_{32}=-\sigma_{41}, \eta_{3}$ and $\eta_{1}$, corresponding respectively to longitudinal ofisets of the waisis in both planes at the IP (we refer to these as offets of the in-plane waists). crose-plene coupling (by analogy we refer to this as an off. set of the out-of-plane waist), and residual spatial dispersion. The waists must be positioned to within some traction of the depth of focus $\beta^{*}$ of the demagnifying optics. and the dispersion $\eta$ must be tuned to leas than $\sqrt{C B^{*}} / \delta_{E}$ to avoid dominating the final spet size.

2. Five terms affecting the angular spread at the IP: $\sigma_{22}, \sigma_{14}$ $\sigma_{31}=\sigma_{42}, \eta_{x^{*}}$ and $\eta_{r^{\prime}}$, determine the band-pass of th. optics. This is illustraled in Fig. 3, which shows the lu minosity $L$ versus $\beta^{*}$ for un energy spread of $0.002(1 / 5$ is taken a \& measure of the overall angular spread $)^{3}$. I the band-pass ia larger than the energy spread, linear of tics dominutes and $L$ drops as $1 / \beta^{*}$. If it is smaller, $I$ is dominated by second order chromatic and geometri perturbations and drops rapidly witb decreasing $\beta^{\bullet}[\mathrm{s}=$ Eq. (1)]. The optimum occurs when the band-pass an! enercy spread are matched. For enerty spreads of 0.00 ' now achieved ${ }^{10}, \beta_{\text {oxt }} \simeq 4 \mathrm{~mm}$. This defines the optic: limit to the luminosity.

Correction elements for the above ten distortions are show in Fig. 1. The waiats are corrected with trim windings on $t w$ of the last quadrupoles just before the IP. and with skequadrupole just upatream. The betatron anjular spread is cor trolled with one skew and two upright quadrupoles in the fis: teleacope. These, combined with the waist controls, form an e fective aoom-lens. Spatial and angular dispersions are correcte by perturbing the $\eta$-match with two pairs of upright and ske quadrupoles. ${ }^{\text {11 }}$

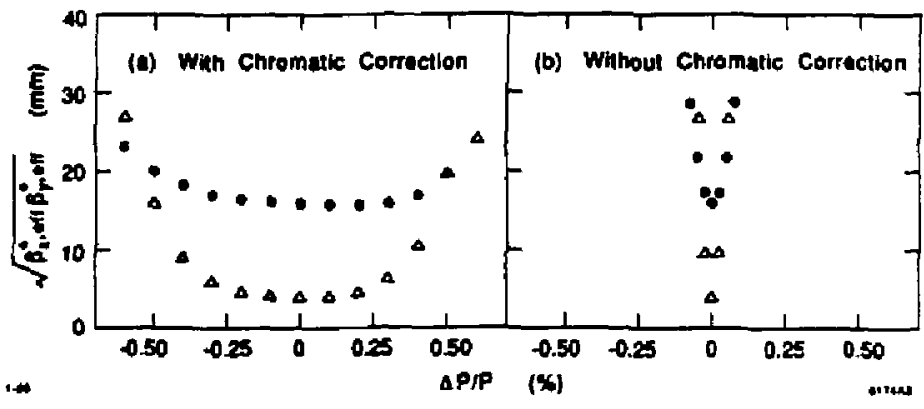

\section{CORAECTION SCHEME}

We defcribe the four-dimentional tranoverte phuseppoe with the unual ${ }^{\top}$ benm-matrix $\sigma$, where $\sigma_{i j}=\left\langle x_{i} x_{j}\right\rangle$. The matrix $o$ has eight free term if the emittancen $c_{f}$ ond $c_{1}$ are set. With the four diaperion functions $\eta_{s}, \eta_{0}, \eta_{\mathrm{r}}$ and $\eta_{\mathrm{y}}$, we thus need twelve parametert to deacribe an arbitrary optical mismatch. For the SLC, equal emíttapces $c_{2}=c_{4}$ are opecified. In this cree, two of the four crouteplane coupling correlations - $\sigma_{21}, \sigma_{a 1}, \sigma_{22}, \sigma_{42}$ - are redundent." With the condi. tion $\sigma_{21}=\sigma_{43}=0$ at the IP, this redundancy tales the form $\sigma_{11}=\sigma_{42}$ and $\sigma_{32}=-\sigma_{41}$.

The tuning otrutegy it deagged for this case and thus involve tea corrections. It can be sbowa that the muximum lumunsity reduction factor, which results in the case of unes: sal

\section{TUNING STRATEGY}

The flow diagrum in Fig. 4 summarizes the experimental a gorithm. Because each correction is coupled to the ones dowi atream, they must be applied aequentially.

Atter matching the input dispersion, the core of the progran is to bring the beam to $a$ focus at tbe IP in a condition such th: the phasespace parameters can be correctly mensured at thpoint. Therefore, the initial set-up bas a purposely enlarged it of $30 \mathrm{~mm}$, with the cextupoles tuaed to suppress the first-ordchromuticity. In addition to reduciag backgrounds, this helps $t$ avoid baving the beam size at the IP dominated by the secon order chromatic and geometric perturbations. It is also a gue of the most probable direction for the angular spread correctio: 


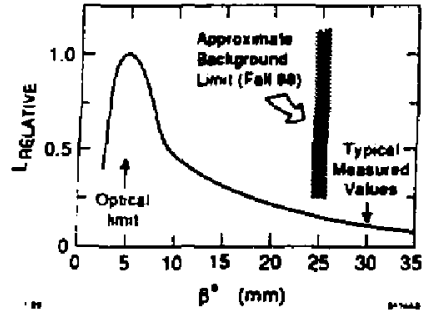

Fig. 3. Optical luminosity versus $\theta^{*}$ in the chramaticily-corrected Final Focus, for an energy spread $\delta_{E}=0.002$. The curve is obtained through a simulation." The approximate backgrosed limit depends on the efficacy of the collimation and shielding, and on the beam intensity.

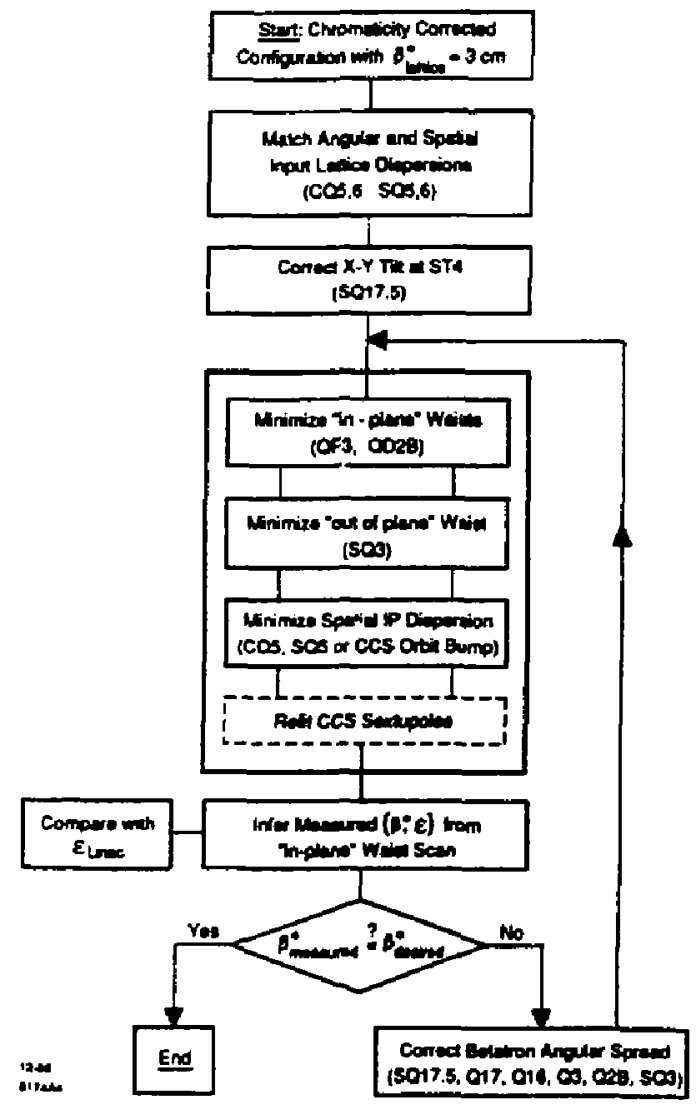

Fig. f. Flow-diagra, summarizing the application of the to linear ortics odjurtments requined to minimuz the IP spol.

In order to decouple the find in- and out-of-plane waist adjustmeats (one of the angular apread corrections) the min. imuzation of the cross-plane 42 correlation is applied fint. ${ }^{22}$ Then the beam is brought to 4 initial focus by correcting the in-plene waists. This, if residua angular dispertios is present, and if the $\sigma_{\text {an }}$ correlation has been imperfectly minimized. helps to diagnose residual spatial dispersion and cross-plane $\sigma_{12}$ and $\sigma_{41}$ correlations in the IP beam size. It thus reduces the num. bet of iterations of waist and dispersion corrections needed to minimize the beam size. In the case of unequal emit tances. the two cross-plane correlations cannot in general be sirnultaneous]y made zero. In this case, it is advantageous to set the out-of plane waist correction to minimize the beam size in the plane with the smaller emittance (typically the vertical plane). Finally: to maintain the minimization of the frat-order chromaticity; the sext upoles are refitted after each major optical adjustment

After this, and unless the angular spread at the IP is 100 large in spite of the $\theta^{*}=3 \mathrm{~cm}$ configuration, scans of the $\mathrm{in}$ plane waists can be used to measure piase-space parameters at the IP. Iaferred values of $\beta^{*}$ are used to calculate angular spread corrections, and of $\epsilon$ to compare with measurements in the rest of the machine.

\section{ON-LINE MATCHING PACKAGES}

An on-line modeling and fitting package is required tor dispersion and betatron angular spread corrections. ${ }^{13}$

For dispersion matching, the input consists of $\eta_{x}$ and $\eta_{y}$ measured at chosen strip-line beam position monitors, and. optionally, at the wire targets ${ }^{14}$ which are used to diagnose the beam at the IP. The data are obtained by recording beam motion correlated with varying the energy in the Linac. The $\eta_{s}$ and 7y value consistent with the model are determined from a fit to the measurements and give $\eta_{r}, \eta_{r}, \eta_{j}$ and $\eta_{y^{\prime}}$ at the entrance to the FFS. The strengths of the four correction quadrupoles in the 7 -match section are then varied to minimize the four dispersion terms at the end of the first telescope or at the IP.

For corrections to the betatron angular spread, the waist messurements at $\beta^{*}=3 \mathrm{~cm}$ are used to specify an initial diagonal beam $\sigma$-natrix at the IP. The $\sigma$-matrix at the entrance to the FFS is calculated from the model. The six quadrupole strengths of the zoom-lens are then varied in a fit to give a new diagonalized $\sigma$-matrix with the desired $\phi^{*}$ at the IP. To help convergence, considerable flexibility is incorporated, including multistep fitting and choice of which $\sigma$-and $\boldsymbol{R}$-matrix elements to include in the $x^{2}$ function to be minimized.

The wajsts are also adjusted through automated procedures, which record beam profiles measured with the wiretargets at the IP, while stepping orthogonal combinations of the two trim windings on the last quadrupoles, or the hearby skew quadrupole. ${ }^{15}$ Estimates of $\beta^{-}$and $\mathrm{c}$ we obtained by fiting

$$
\sigma^{2}=c \beta^{*}+\frac{1}{\beta^{*}} \Delta f^{2}
$$

to the in-plane waist data, where $\Delta f$ is the displacement of the weist at the IP along the beam direction. Because the squares of the beam sizes vary parabolically, the optimal correction is found, by symmetry, even if the minimum beam size is less than the wire size.

\section{INPUT DISPERSION MATCH}

Figure 5 shows mensurements of the lattice dispersion. mea eured in the n-match, First Telescope and chromatic correction section, before and afles correction. Variations serve to diagnose changet in the set-up of the Arc and are usually correctable. ${ }^{16}$ In this Figure the borizontal (upper figures) and vertical (lower figures) dispersion is shown before (left) and atter (right) correction, using an exumple of data taken during the 1987 SLC commissioning. Note the changes in scale. In each figure the error bars on the solid curve show the results of simultaneous dispersion measuremeots on the electron beam using BPMs in the SLC North Arc and the n-Matching. First 
Telescope and Chromatic Correction Sections of the Nortb Final Focus. "The solid curve is a series of strajght lines joining these measurement points. The dotted curve is a series of straight lines joining points showing the corresponding value of the design dispersion at the location of each of the BPMs. Alter corretion the measured dispersion in the $\eta$-Matching and First Telescope sections is very close to the design values. Such measurements are performed routiatly to monitor the match. They are usually repeated to average out trajectory fluctuations during the messurement which can mimic dispersion mismatch. The match bas been observed to be stable over periods of days to weeks.

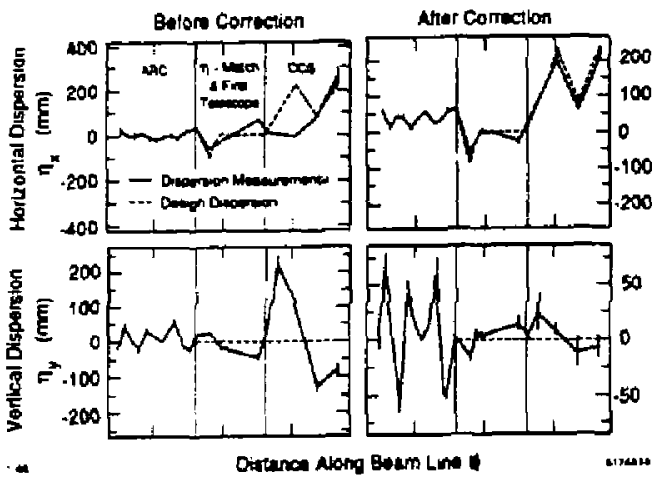

Fig. 5. The First Telescope dispersion cortection.

\section{CROSS-PLANE COUPLING CORRECTION}

Figure 6 shows the correction for the tilt in the spot on a phosphor screen near the Final Triplet (ST4 in Fig. 1). A tilted spot at that proint corresponds to a finite $\sigma_{12}$ corselation at the IP. This is Jone manually by adjusting the skew quadrupole in the first telescope. The correction is difficult to set accurateiy and reproducibly because of changing beam tails and saturation effects on the screen. A fit of the $\sigma_{13}$ correlation coefficient using the digitized profle may improve this. The svalable correction range is large, but the practical range is severely limited by perturbations caused to the trajectory of the opposing outgoing beam, which must pass off-axis through the skew quadrupole be fore reaching the final bean durnp. $A$ procedure for controlling cross-plane coupling within the Arc has been developed, which mitigates this problem substantially. ${ }^{17}$ Such cootcol bas reduced coupling in the lattice to about $50 \%$ and bas brought the FFS skew corrections to acceptable values, although this is not fully stable and depends on the ratio of emittances at the Linac exit, as described above.

\section{WAIST ADJUSTMENTS AT THE IP}

Figure $\boldsymbol{i}$ show's an example of a waist sead (in the vertical plane). Such scans ase done coutinely, allowing minimum beam sizes of 3 to $5 \mu \mathrm{m}$ to be attained.

\section{DISPERSON CORRECTIONS AT THE IP}

There can be significant residual dispersion in the IP beam size. even after the inpul dispertion bu been mitched, due to imperfections in the FFS latice or in the beam trajectory, or from energy-position correlations is the phase-space at the ead of the Linac. Dispersion from the FFS can be mentured by the online package described earlier. Corrections with the four quadrupoles in the $\eta$ maleh section se practical for moderate dispersions ( $\eta / P \leq 2 \mathrm{~mm}$ ). Larger digpersions, bowever, can require extreme corrector strengths which, in turn, also distort the betalron phase-space
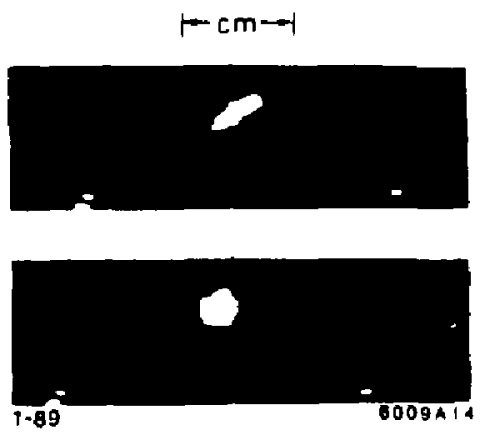

Fig. 6. Approsimate correclion of cross-plant coupling in the IP angular spreads, looking at the lult in the beam shape on a screen al the high-9 point in the system.

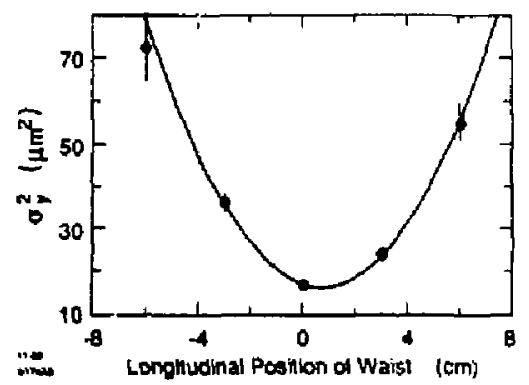

Fig. 7. Minimization of the tertical IP beam sise by displacing the vertical waist with an orthogonal combination of trin urindangs in the last quadrupoles.

A compiementary scheme for empiricaly minimizing residual spatind dispersion at the IP makes use of closed steering bumps in the chromatic correction section. Such orbit distortions generate spatial dispersion at the IP. through the firstorder chromaticity of this section. For the range of interest, second-order chromatic and geometric perturbations remain small. An example of successfully applying this method to min. imize the spot at the IP is shown in Fig. 8 . In combination with the lattice diapersions measured in the first telescope and at the IP, this method has allowed sepsration of lattice dispersion generated in the Arcs and in the FFS. Since the spatial dispersion introduced by this bump to minimuze the spot size has coincided with the previously measured lattice dispersion at the IP. it has been possible to pul an upper limit on beam dispersion at the end of the Linac.

\section{getatRon PHASE-SPACE DIAGNOSTICS AND ADJUSTMENTS AT THE IP}

History plots of $\mathrm{t}$ and $6^{*}$, estimaled fTom in-plane wasst scans performed after iterating the waist and disperston correc tions to minimize the spot at the [P. ate shown in Fig 9 .

The emittances $e_{x}$ and *, were mostly iarger than nomual and retlected, in most cases. lasger than nominal balues in the Linac. The $\beta^{*}$ values were larget than the experted optimum of $\beta_{a p t}^{*} \simeq 4 \mathrm{~mm}$ needed to optimuze the 'umunosity, and resulted from requiring a small enough angular spreart to inatmys beam 


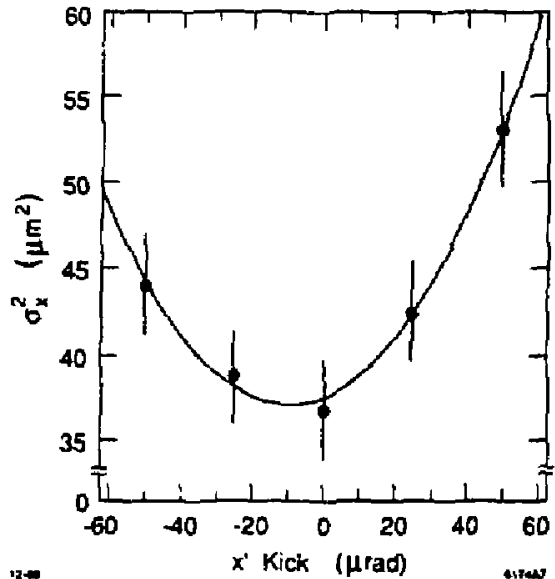

Fig. 8. Correction of residual dispersion at the $I P$. by minumizing the spot size with a closed dispersion-generating trajectory bump in the CCS. The parameter a'KICK is the magnitude of the kick applied by a sleering dipcle located at the upstream end of the CCS. A corresponding dipole at the downstream end is useo to close the bump.

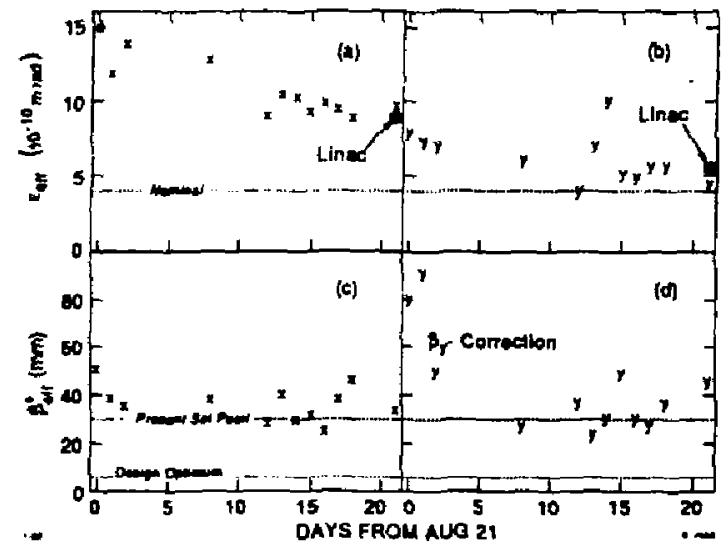

Fig. 9. Hisfory plots of the amittances and $\beta$ functions inferred from in-plare waist-scans at the IP. The effect of an initial attempt towerde redueing the pertical $\beta$-funclion is indicated. As can be seen, al the end of the ren, emittance mensure. ments performed simultaneously at the end of the Linoc and at the IP gave conastent results.

tajl-induced backgrounds in the lat quadrupoles. In come casea, lager than nomind effective $e$ and $\sigma^{*}$ value were also obtained becaune of an imperfectly corrected phasespace at the JP. The data in Fig. 9 are therefore generally upper limits of actual val. ues.

A first attempt to eniarge the (vertica) betutron angular apread is indicaled by an anow in Fis. $9(d)$. The effect from this wan clear but smaller than expected, and may beve been partially ofiset by an upitieam variation. Such adjustmenta will have 10 be iter sted in order to reach the expected optimum value of $g_{0 \mathrm{~A}}^{\circ}=4 \mathrm{~mm}$.

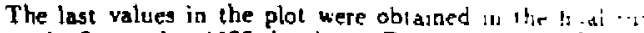
before the September 1988 shutdown. Dispersion al 'he. [l' ar. erated by trajectocy errors and misalignments was measured all minimized with the bump technique described above Ths. anil several iterations of the waist corrections. resulted in crutlanw elose to the design value in the vertical plane and too larde $n$ i h. horizontal by a factor of three. This was consistent with ntw surements performed simulaneously at the end of the Linar.' showing that the final beam sizes at the IP were not donunatri by chromatic effects, and that the residual cross-plane couplitix from the Arc did not significantly enlarge projected emullances At that time, the linear phase-space at the IP was thus corter ils estimated "-om these measurements.

\section{CONCLUSION AND PROSPECTS}

The experimental algorithm developed for,the FFS has en abled beams focused at the IP with 3 to $5 \mathrm{~mm}$ transverse sizes to be attained, and the various contributions to the restdual beant size to be distrosed.

The residual beam size is presently limited by the larger than optimal $\beta^{*}$ (dictated by detector backgrounds) and by the somewhat larget than aominal Linac emittance. The reduction in luminosity from this is about an order of magnitude. In ad. ditjon, a small loss in atrainable luminosity arises from not fully correcting the cross-plane coupling in the case of asymmetric emittances. This loss tas be up to about $25 \%$, with the current emitlance ratio of one to three.

In the next rud, a new collimation system will be avajlable at the end of the Linac which, combined with the existing slits and with additional muon shielding that has been installed in the FFS tunnel, will enable beam tails to be cut mote efficientl: This, coupled with progress in majutaining a nominal phase. space al the jnjection to the Arcs, and in reducing the 3.function at the IP, should easble the optical limit to the luminosity to be reached.

\section{ACKNOWLEDGEMENTS}

We wish to thank the very large number of people. from SLAC and elsewhere, who bave helped to make all shis possi. ble. In particular, we wish to acknowledge the contributions of K. Brown, R. Erickson, T. Fiegutb and J. Murray, who init. ated and finalized the deaign of the Final Focus optics, and of L. Sweeney, who contributed to much of the ronttol software. Fieguth and Mutray also initiated the work on the experimental tuning algoritbm.

We also wish to scknowledge tbe support and enthusiasm of our colleagues from the SLC. MarkII and Operations Groups. who helped carry through many of the measurements described here.

In addition, two of us (W. F. and T. L.) were supported

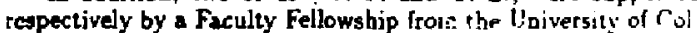
orado and by a grant fron the Mex hade Foundation:

\section{REFERENCES}

(1) SLC Design Handbook, December 1984, Stanf.rd Linear Accelerator Center

(2) K. L. Brown, A Conceptual Design of Final Facus Systems for Linear Colliders, SLAC-PUB-4159 (Jure 1987 ).

(3) P. S. Bambade, Beam Dymamics in the SLC Final Focus System, SLAC-PUB-4227 (June 1987).

(1) P. S. Bambade, Recent Progress of the Stanford Lineur Collider, SLAC-PUB-46I0 (April 1988).

(5) D. L. Burke et al., Beam Colhmation and Detector Back. grounds at $e^{+} e^{-}$Linear Calliders, these proceedings

(6) W. B. Atwood et al. Otservation and Coutroi of Emut. tance Growth in the SLC Linac, these proceedings 
(7) K. L. Brown et al. SLAC-91, Rev. 2. May $197 \mathrm{i}$.

(s) P. S. Bambade, Number of Dimensions of Optical Cosrecuon Space in the SLC Find Focus System, collider note in pteparation.

(9) J. J. Murray and T. Fieguth, MURTLE, private program.

(10) E. J. Soderstrom et al., Fast Energy Spectram and Thans. zerse Bcam Profile Monitoring and Feedbeck Systems for the SLC Linac, these proceedings.

(11) J. J. Murray et al., The completed design of the SLC Final Focus System. SLAC-PUB-4219 (February 1987).

(12) P. S. Bambade, Orthogonality of Final Waist Corrections at the IP of the SLC, CN-369 (October 1988).
(13) C. M. Haw kes and P. S. Bambade, First Onder Oplical Matching in the Final Focus Section of the SLAC Limfor Collider. SLAC-PL'B-462! (May 1988).

(14) D. L. Burke et al., Measurements of Cross Sectional intensity Profiles of Beams at the $e^{+} e^{-}$Interaction Point of the SLC, these proceedings. and submitted to Suclear Instruments and Methods.

(15) N. Phinney et al, An A stomated Focal Point Positıoning and Emittance Measurement Procedure for the Interaction Point of the $S L C$, these proceedings.

(16) T. H. Fieguth et al., Dispersion in the Ares and Sptcial Sections of the SLAC Linear Callider, these proceedings.

(17) P. S. Bambade et al, Wirefix - Or Harmanic Corrections in the SLC Arts, these proceedings. 
VIII. REPORT ON GENERAL STATUS 
VII. "Recent Progress at the Stanford Linear Collider"

This article describes the main advances in the commissioning of the SLC, during the spring, summer, and winter 1988. The seminar concentrates on the start-up of the arcs and final focus, which were the main activities in those periods. 


\title{
RECENT PROGEESS AT TFE STANFORD LINEAR COLLIDER'
}

\section{P. S. BAMBADE}

Stapford Linew Accelerator Canter, Stanford Univeraity, Stunford, Culfornia 91309, USA

\begin{abstract}
A ituru report on SLC commimioning is given, with special emphusis on recent progreas in the Arce and Final Focus.
\end{abstract}

\section{INTRODUCTION}

The Stanford Linear Collider (SLC) has two main goals [1]. The frat is the production of high luminosity electron-ponitron collisions for studying the physics of the $Z^{0}$. The second is to test linear colliders as a new approach towards future high-energy machines.

Circular colliden, where counter-rotating electrons and positrons are atored, are not easily extrapoleted to very hich energy becauve of copioun anchrotion radiation emitted in the berici. Both size and cost weale [2] $4 E^{2}$ for un optimized design. Linear colliders aroid this by acceleratine beame in linacs to the

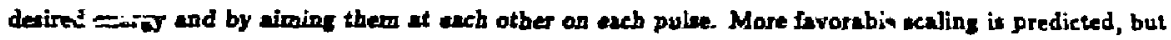
not fully etablished, and new nroblems exist. Beajdes the ecceleretion mechanism, a upecial chailenge in the wall collinion point benm vire needed to male up in the luminanity for low croning rate. At the SLC, it is about $2 \mathrm{~mm}$ is radiu. Both sluborate oplice end emitunce reductions vis radiation damping are

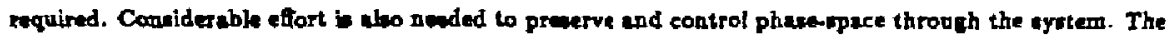
SLC, cervins to explore ouch problems, is an important learning experience.

\section{PROJECT OVERVIEW}

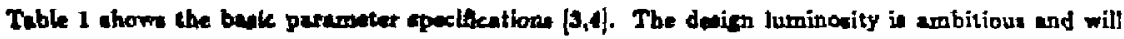

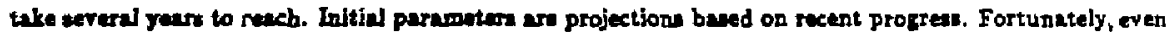
a thourandth of the desipn luminaity allows to do wome interesting phyaics.

Table 1. Buic perameters for the SLC.

\begin{tabular}{|c|c|c|c|c|}
\hline & Duifn Gosl & Initial Gosl & Aehieved & Units \\
\hline Benm eneris at IP & 80 & 40 & 46 & GeV \\
\hline Beam eners at end of line & 51 & 47 & $63^{\circ}$ & GeV \\
\hline Eietron at entranes of arca & $7 \times 10^{30}$ & $10^{10}$ & $3.5 \times 10^{10}$ & \\
\hline Ponitrons at entrence of ares & $7 \times 10^{10}$ & $10^{10}$ & $0.0 \times 10^{10}$ & \\
\hline Depaition rala & 180 & $120 \mathrm{Es}$ & $10 \mathrm{Fz}$ & $\mathbf{H z}$ \\
\hline $\begin{array}{l}\text { Normalined tranverm enitines } \\
\text { it end of line (eletrons) }\end{array}$ & $3 \times 10^{-1}$ & $10 \times 10^{-1}$ & $3-10 \times 10^{-8}$ & rad-m \\
\hline Spot radiun at IP & 1.6 & $4 \mu$ & $\sim 4$ & $\mu \boldsymbol{m}$ \\
\hline Luminesity & $6 \times 10^{30}$ & $6 \times 10^{27}$ & - & $\operatorname{cm}^{-1} \operatorname{aec}^{-1}$ \\
\hline
\end{tabular}

-Work supportad by the Depurtment of Energy, contract DE-ACO3-765F00515.

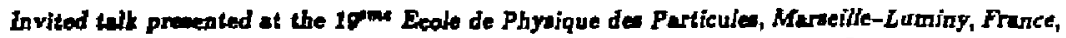
September 7-11, 1987 and at the CERN Aecelerator Sthool, Berlin, Germuny, Seplember 14-25, 1987 
Figure 1 thowe the entire SLC complex. Two electron bunches are generated and co-accelerated to $1.2 \mathrm{GeV}$ in the Injector [5]. At $200 \mathrm{MeV}$, they are joined by a positron bunch. The three are then injected for cooling into two Damping Rings [6], from which they are extracted before the next linac pulse. The existing SLAC Linac has been operaded [7] and co-secelerates them to $50 \mathrm{GeV}$. The $6 \mathrm{~mm}$ equilib:i:-it ring bunch length is compresped to $1.5 \mathrm{~mm}$ beicoe injecting into the Linac in order to misimize vakefield effects there. The Iat electron bunch is ejected onto turget at $33 \mathrm{GeV}$, to produce $[8,9$ ! positrons. These are returned along the length of the 'jinac to the $200 \mathrm{MeV}$ point in the Injectot. At the end of the Linac. electrone and positrons are brought into collinion through two Arca $\{1,10)$. The Final Focus System :11-13;, straddling the interaction area, Jemannifies and steers the bexms into collision.

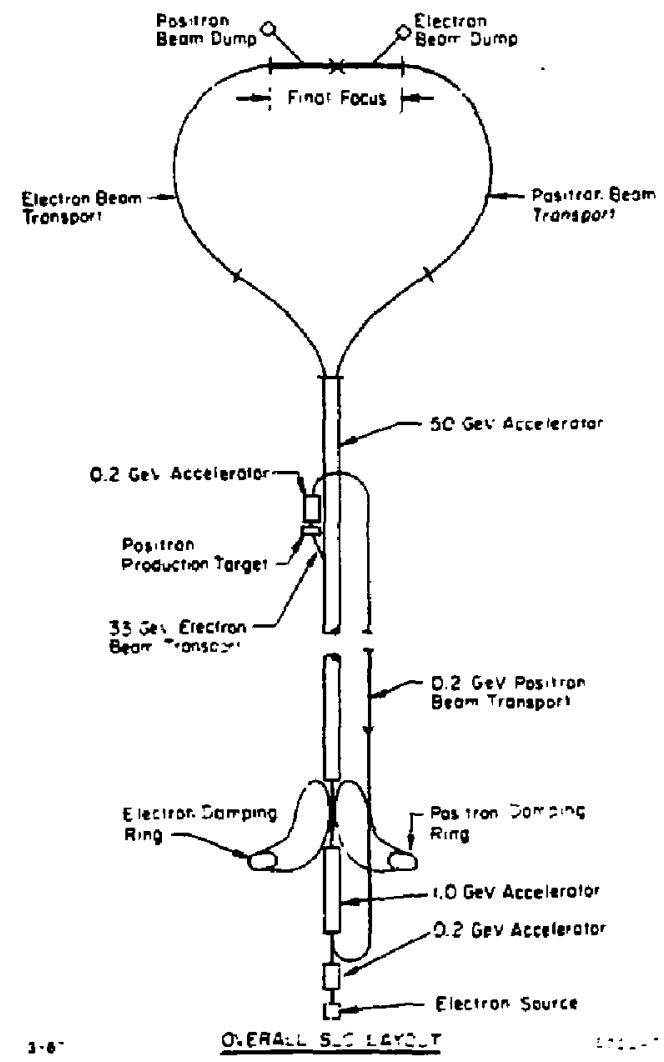

Fig. 1. The Stenford Linear Collider (SLC).

Commingioning and lexte of avcceasive stages have been ongoing since the Fall of 1981 and throughout the construction period (Fall 1NBd to Sprins 1987). Subsequently, rouch work has been devoted to the newly intelled Arce and Find Focm System, with continuing improveroents upstseam in the Linac, the Electron and Posiaron Sources, and in the Damping Rings. In this paper, we revies the Arcs and Final 


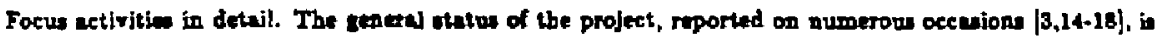
briety summerised.

\section{LINAC AND SOUZCZS}

Injector [5]

The Electron Source and Injector are apecifed to provide two bunchen of $7 \times 10^{10}$ particles with * momentum apresd within the Dumping Ring accoptance of $\pm 1 \%$. Invariugt emittance mut be amaller then $180 \times 10^{-6}$ mad at $120 \mathrm{~Hz}$ oparation. Theee goul are mot asily for $8 \times 10^{10}$ particles per bunch and the nystem is tabilized throuth computer conlrolled fuedback.

\section{Damplog Ring [6]}

The Darping Ring have provided the decion in rutient emittance of $3 \times 10^{-1}$ mrad at 1.2 GeV. Both fing are operational but ueful current extracted is limited to about $2 \times 10^{10}$ particles per bunch becture of bunch lenthening. The origin of thl efret in exensive longitudinal impedence from diecontinuitien in the vecuum chamber. It in posible to extract Inrger currents, but the bonches cat then not be comprested in the ring to lince tranport becaue of limited aperture. Short-term tres here Included opening up thi aperture, and inducins quadrepole eacilletion to precompres the bupch within the ring juet before extraction.

Some redpetion of the impedenes ean be echiowed by tnatalling alerves injide the bellows, to amooth out the tranitions thot.

Such fxe ar expected to hulp bring the corrent up toward the delen value. In the thort term, the aystem is adequate for operation at $10^{10}$ particles per pulee.

Pooltron Bouree $[0,9]$

At $33 \mathrm{GeV}$ in the Linec, the wcond electron bunch is ejucled onto w-Re target to produce positron.

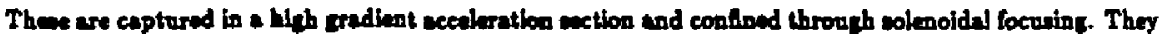

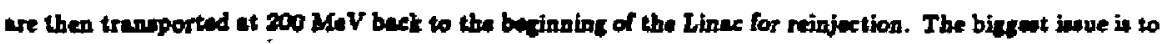
increase the jlatd of demped positron rolnjeted into the Linec per electron incldent on the terget. Prior

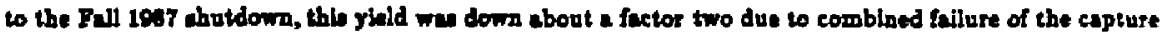

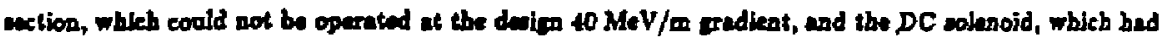

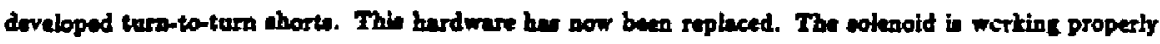

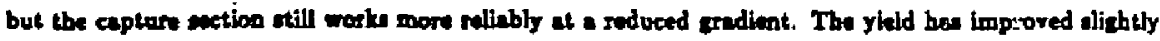
but her pot yet been pushed to the derign value $1 \times 10^{10}$ positros buncher bave been obtained at the end of the Linec.

Lboe [7]

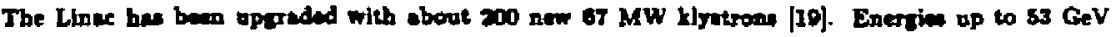
have been mesured, but ranning-in in at $47 \mathrm{GeV}$ to produce collition at the $2^{0}$. Co-acteleration of $10^{10}$ electrons and poritron is fairly ear and comonon atomed bean pridence is operations. The energy upread can be minimised to .2 to .3\%, and work it ongoing to improve launeb and energy feedbuck 
atabilization into the Ars. Optical matching, importent to preserve emittances, is underway both at the injection and throughout the lattice. A klystron management program, enabling automatic ecaling of the lattice 20 malch to a vering tystron population, i beint teated. Inveriant emittance between 3 and $10 \times \mathrm{jO}^{-5}$ mind are at preseat obtained at the end of the Linec for both beams.

\section{ARCS}

\section{Summary of Optica Could $[1,20]$}

The Ares are devifned to bend the beams without significant dilution of tranverse phase-space. Two mechaniam muat be counteracted.

The fint efiect result from synchrotron radiation. The photon, emitted al random, cause energy Buctuations. Lower energ purticles are bent more and follow survo with whorter average radius. This disperaes their trajectorie ineoherently which enhence horisontal phaseapuce. Such trajectories execute betatjon oxeillations in the quadrupole intice. To minimize the crowth, both photon emission rates and oscillation amplitudes mut be amal $[21,22]$. This is achisved by making the bending radius large and the betatron period sbort, through tight focusing. As can be thown [1], the growth is proportional to T/p/ot, where $T_{p}$ is the betutson pariad and $\rho$ the average redius. Alternating eradient trunbort modules with the lowent pourible feld compatible with the SLAC vite are therefor wed. The pucking factor in muimized using combinad function magnts, and the lattice chosen to minimize the average invariant amplitude of the disperaed arcillations. For a FODO array, the optimum [23] cell phase-shift to minimixe emittance

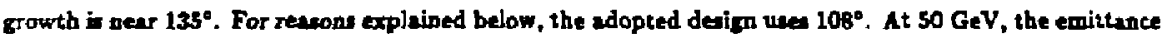
added in one parage is |1\} $1.5 \times 10^{-10}$ rad-m, or one-balf of the derign value.

The exond mecheniar phese-space dilution arise through retidual energy spread resulting from the bunch lenth and the eccelerating Linsc RF [24]. Becuuse of energ dependance in the focusing, or chromaticity, optical divtortions from pradient errors are not imaged coherently. For example, an of-

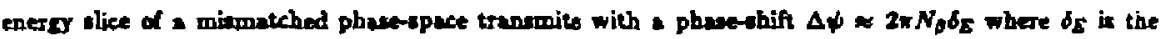

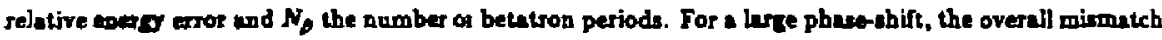
everaged over all enerites boses its phase relation to the input. The efrective volume occupied by the

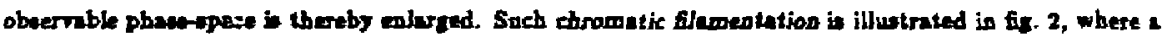
normaliend phenepace with ares one bat amplitede two distortion cradually fille up as area of two. With $N_{p} \simeq 70$ in the Arc and for $\sigma_{\Sigma} \simeq 0.5 \%$, the spread in betatron phaes at the output in about $\sigma_{\downarrow} \simeq 0.7 \pi$.

This effect cen be controlled in two ways. In an active approsh, it is reduted with careful energy spread minimisation [7] and good trajectory and optica matchine lnto and through the Arc. In the adopted panjue

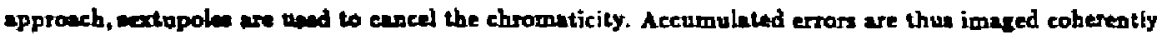
and the find corretion can be concentrated in the Find Focw. From a purely optical standpoint. this eases requirementi on upatrem coatrol. In prectice and as we shall explnin, much upstrenm contral is itill necurary to maintion datectable luminotity.

Sextupole were introduced by thaping the combined function mithet poles [10]. For the horizontu] aptice, the vertical componenl of the mometic field may be expreased a:

$$
B_{3}(x)=B_{0}\left(1-Q x+S x^{3}\right)
$$




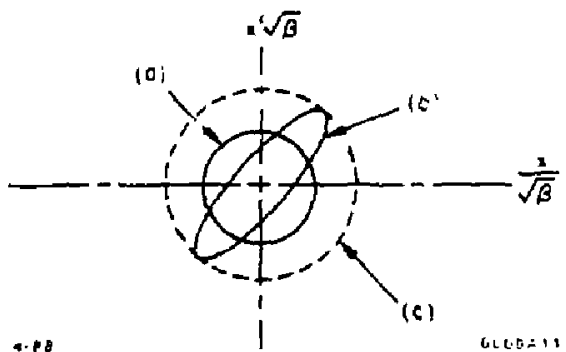

Fia. 2. Nominal phaso-apece (a), distorted by optical mismatch (b). Elaments (c) into a inger ares when not correctint the chrometicity of the lattice.

where $Q$ and $S$ are the quadrupole and wextupole atrength. The sextupole provides additional focusing for offeners and off-axia raye with $I=6 x+b_{20}$, where $\eta$ is the dispertion function, wistch suppresses the chromaticity if $2 S \eta=Q$. Since sine and cosine-like components are equivalent modulo $\pi / 2$ in $*$ repetitive lattice, only one faroily per plane is needed. Additional term in $x^{2}$ and $c$ for rays solely off-axis or off-

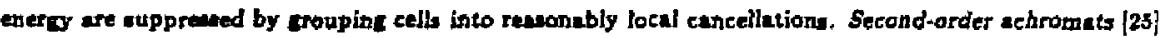
achieve this by pairing sextupoles * phae-shift apurt into superperiode with the amallest poevible multiple of $2 \pi$ compatible with the cell phese-ahift. In the SLC Ares, each ouperperiod is $6 \pi$, conistine of ten $103^{\circ}$

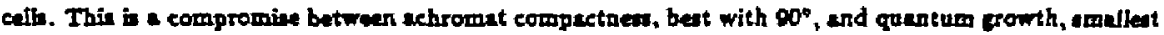
Dear 135".

The price to pay for using sextupole is a battice cennitive to misalignmente. A poorly controlled trajectory generates exwet and akw gradient errort throuth the text upole which add to megret imperfections [26] und enhance optical dintortions. This lesd to atringent magnet to magnet alignment tolerance [20] of about $100 \mathrm{\mu m}$.

The aficacy of the chromatic corraction was tated by comparine set of betatron oscillations at two

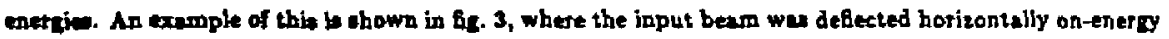
and $100 \mathrm{MeV}$ ofenerg. Overlayins the plots show no phase difierence.

\section{Nonpluner Geomotry}

Commiationing revalted another problem. Although the bean [10] we steered through the Noth

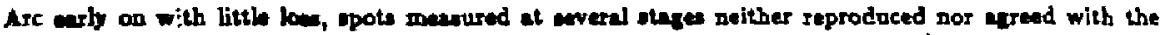
deipo, and the ' and injeted in the Final Focul for hardwere tate would not fit in the aperture easily. At timm, low loo ippeared in the Arc, pointing to supected machenical problem with the vacuum chambar or the magnet movers used for ataering. In attemptn to probe available aperture with beam, it

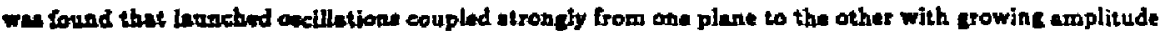

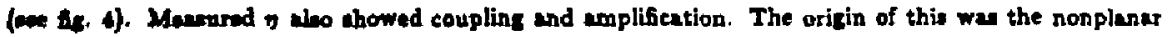

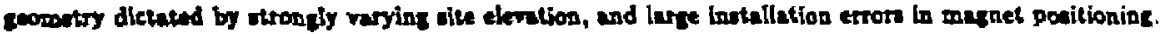
Achromate ware rolled at their intarince wo follow the tarrain, and bis couplings correlated with the largest rolle.

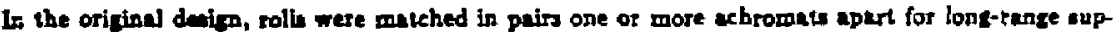
preasiot of the coupling, and this was ansitive to deviations in the 6 nt phasesdvance, from byse. atic 


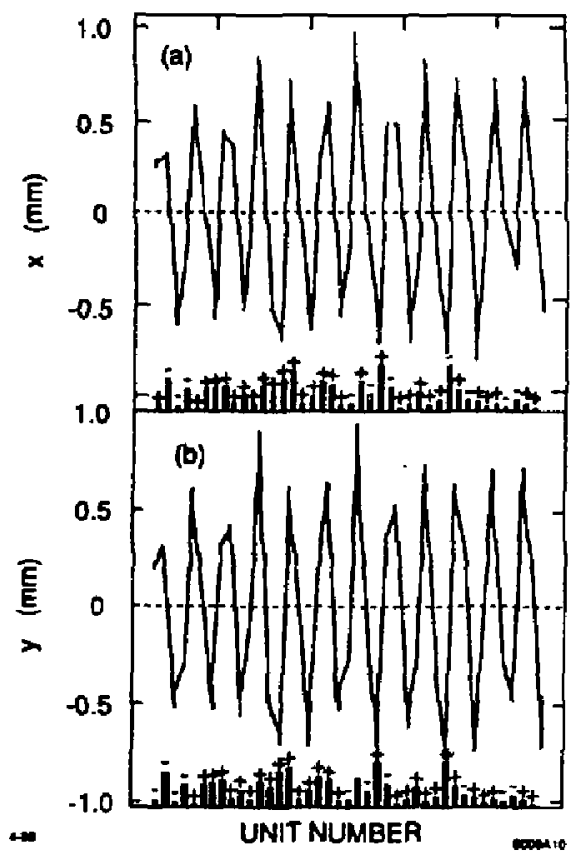

Fix. 3. Betatron occillation at the end of the Arc, on-entrg and $400 \mathrm{MeV}$ off-energy. The chromatic correction ouppresses any phase-shift.

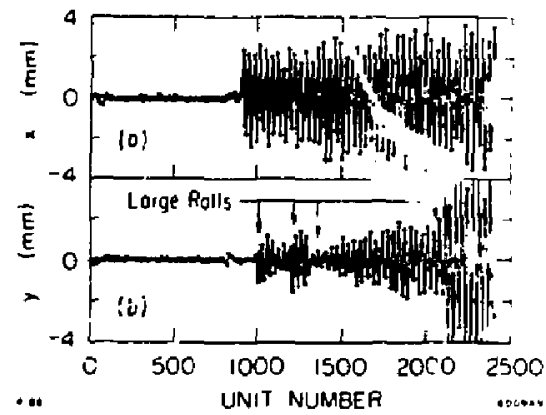

Pis. 1. Crace-coupling of betatron otcillation in the North Arc. Lerge coupling occurs at the largent rolls.

gredieat erron. Such erron inltially exceeded the opecified design tolerances [1]. Eflects can be large as crou-coupling oucillation each time see out of phese optica in the other plane. At a $10^{\circ}$ roll, the conpling [27] can be I00\%. Overall senvitivity of the betation size is thown in fig. 5 from TRANSPORT [28:, for iybtematic errors of $3^{\circ}$ per cell in each plane. Measured errors were amaller and accounted for a factor three to four overall growth. This wa not sufficient to explain the large spots observed in the Final Forus, Indicating that the Linec phasaspace wae not fully controlled. 


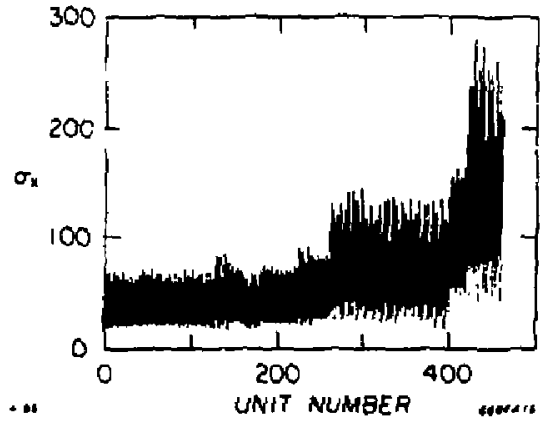

Fix. 5. Growth of betatron upot sive in the North Are, for matematic erros of $3^{\circ}$ per cell in esch plane.

Pbave-Fix and Roll-Fix

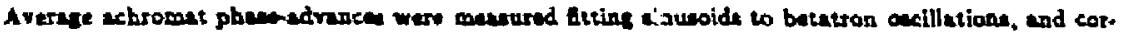
rected by phyaically moving mapnta, and by combinins trim windings in ach achromat and a global

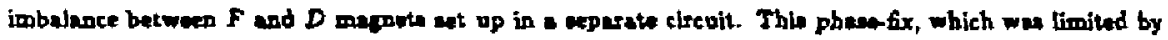

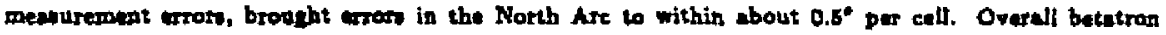
zrowth wa reduced to a fetor 2 and $\eta$ wes enentilly matehed. The Booth Arc, only partially correcud,

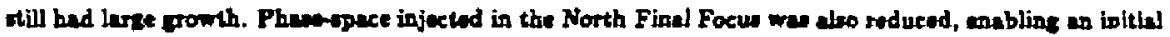
opties propram for amall IP spote to proesed, but remainine growth, otlll amplifyine veriations, would oflen rowlt in ancorrectuble cane.

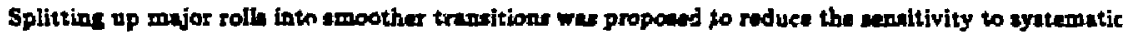

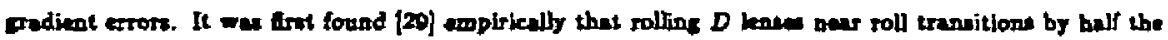
total smount supprand craneoupling of lal tice $\eta$. For the betatron motion, in epproximute correction echeme was found by splitting tolls in thre parta, ach a celi apart and with sagnitudea matifying:

$$
1-1 e^{-i 3+/ 3}+6+10+1 e^{i 3-1 / 3}=0
$$

a for a matched trajetory bump. Figure 6 illustrates the combined roll-6x transition and relative roll

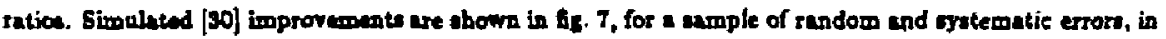

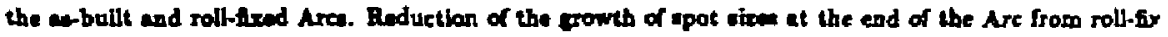
in s fuctor 1.5 to 2 .

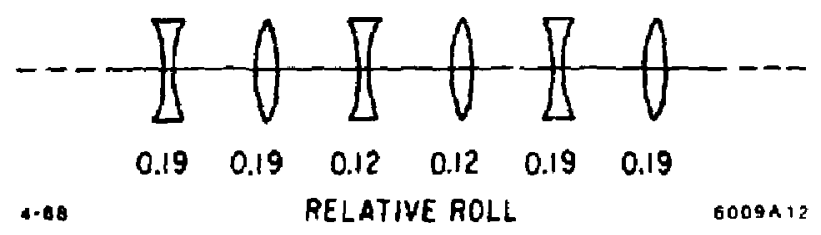

Fis. R. Rollefe trandition. 


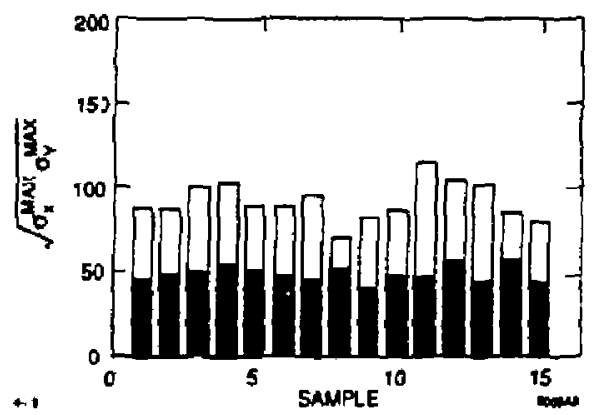

Fig. 7. Growth of setatron size at the epd of the Arc, lor 24-buill (white area) and roll-fixed (shaded ares)' 'stise.

\section{Prevent Statu and Plens}

Both Ares have been soll-5xed and recomminsioned. In the South, alightly moophes transition [31] is installed. With proper leunch, $\eta$ is matched throuchout. The rensitivity of the betatron motion from systematic cradient erron in reduced, but randon ereet and akew quadrupole erron till cause some blowup : ctom-coupline, although more gradual in nature [me fig. B (e)]. Cure involve empirically varyiog pheses in troubled aren to reduce the coherence in the buildup, wuch we sune are adjuated away

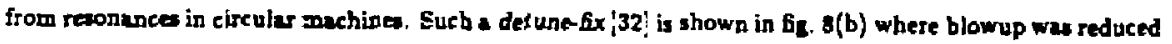
by disconoecting the FD-imbalence, thus cawing sbout a $1^{\circ}$ per cell difference between $X$ and $Y$ phuses.

Nother planned [33] cure consists of exciting baranonic [31,35] cradient perturbations with \& pattern of trim wirding, to ouppres damacine Fourier components in the erron at twice the betatron frequenty. Although this will be required for the final optimitation, beams are now soutinely transported to the Final Focus with distortions which cas be absorbed 1 \% optical malching there.

\section{FINAL FOCUS}

Summary of Optice Gouls [11-15]

The primary coul is to focus both beams to a small tranoverne size of about $2 \mu \mathrm{m}$. This would be eary for amall enough traneverse emitinted and energe tpreads. The limiting effect is the chromaticity of

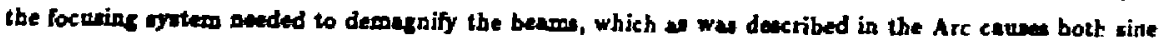
and cosina-like trajectorion to be ohifled for different energies. For a simplified Final Focus 'we 5e. 9).

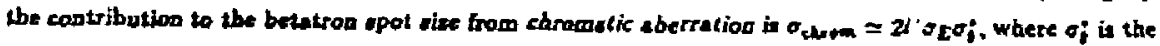
IP angulur aise and $f^{\prime}$ the diatance from the principal plane of the fina) lens syatem to the IP (o factor

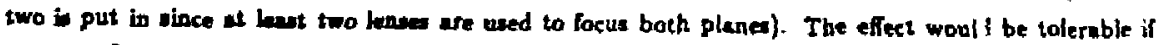
$\operatorname{co} E \leq \sigma_{2}^{2} / 2 \alpha^{\circ}$. This is not the tase in the SLC Final Focus, whete $\sigma_{2}^{*}=1.5 \mu \mathrm{m}, c=310^{-10}$ mat, $\sigma_{E}=0.002$ and $\sigma^{\circ} * 5 \mathrm{~m}$, amounting le $\sigma_{\text {edrom }}^{\circ}=4 \mathrm{\mu m}$.

The raduction in lumionity, computed [36] by averaging the usual expression over the two beam's energy distributions, is showa in Ac. 10 (dotted lines) versus $\beta^{\circ}$, for the a-built Final Focus. With os $=$ 0.002 and $D_{\mathrm{cec}} \approx 1.5 \mathrm{~cm}$, the luminosity lo reduced by $\&$ factor 3.5 . 



Fic. B. Gromth of a bettron oncillation in the South Arc, before ( $\bullet$ ) and after (b) detune fix.

A Chromatic Correction Section (CCS) in introduced upatran of the find lene to eancel this effect. In a timplified CCS, two dipole of strength $B$, exparated by $2 /$ cers are imaged bs a qued with focal length

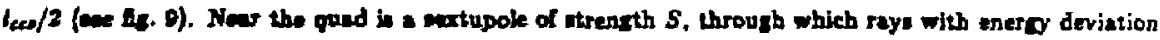

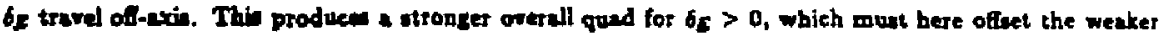
becuing in both find and CCS quads. The lurgest contribution to the ehrometicity in from the final lens. Equation it $w$ the afect trom the eaxtop , we find that $S \propto R_{l} / M B$, where $M=l^{*} / L$ and $R_{l}=I^{*} / l_{c t,}^{2}$.

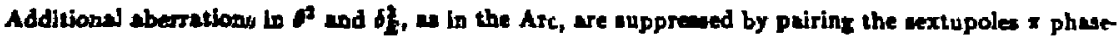

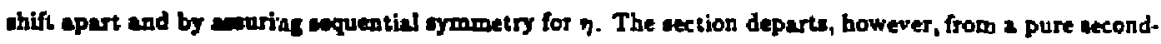
order schromat througle tho benda, piaced where engolar apreads are large to minimize aynchrotson ra-

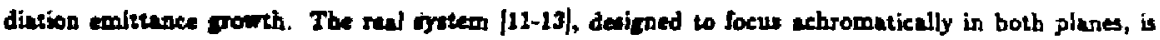




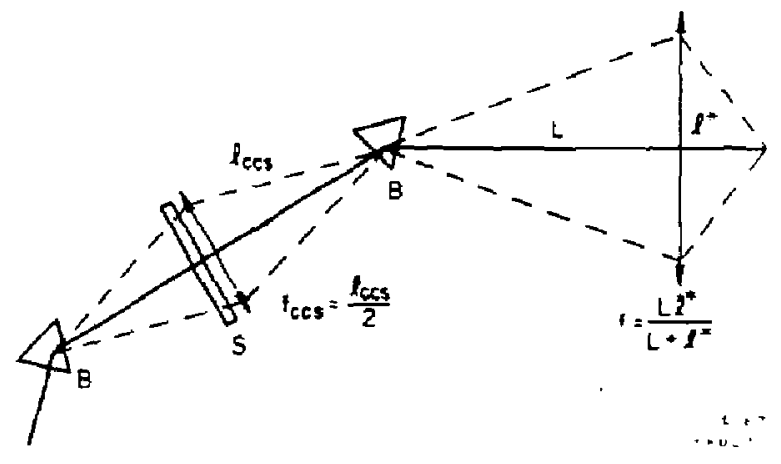

Fig. 9. A simplifed Final Focus Syotem.

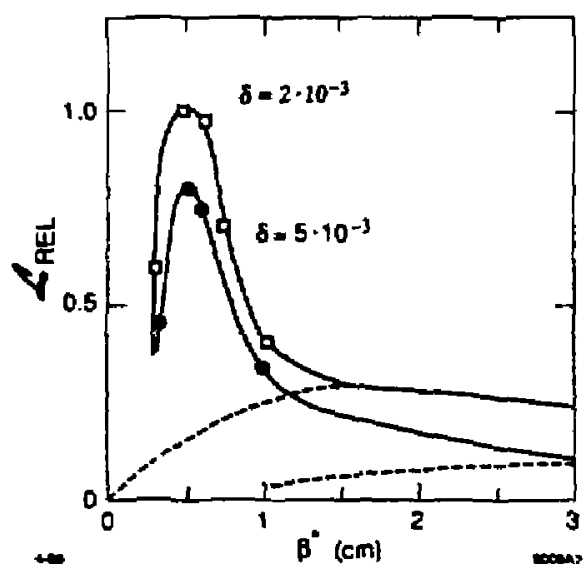

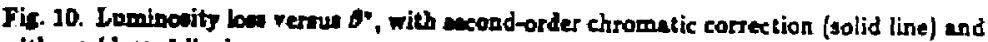
without (dotend line).

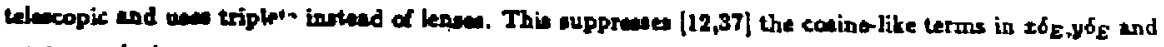

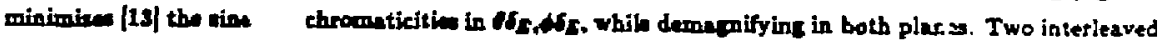

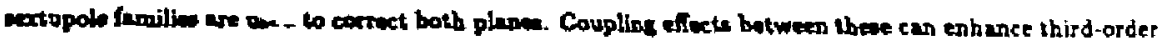

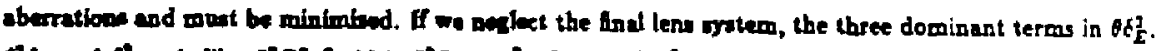

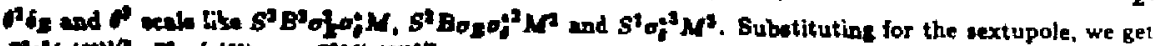

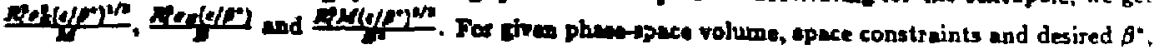

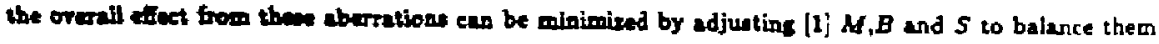
out.

The ovarall wect of the chromatic correction is ohown in 68. 10 (nolid line). Fom MIRTLE ;38') As

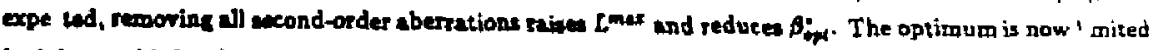
by blever third-ordes teron. As it is more peaked, it is aluo mare cenvitive to proper matching. 
The entire aytem is abown in fic. 11. Two additionsl sections are included to match the Are $\eta$ and $\beta$-functions. Extraction of the opposing beam is also provided, in the $\beta$-match section, through a pulsed megnet and reptum.
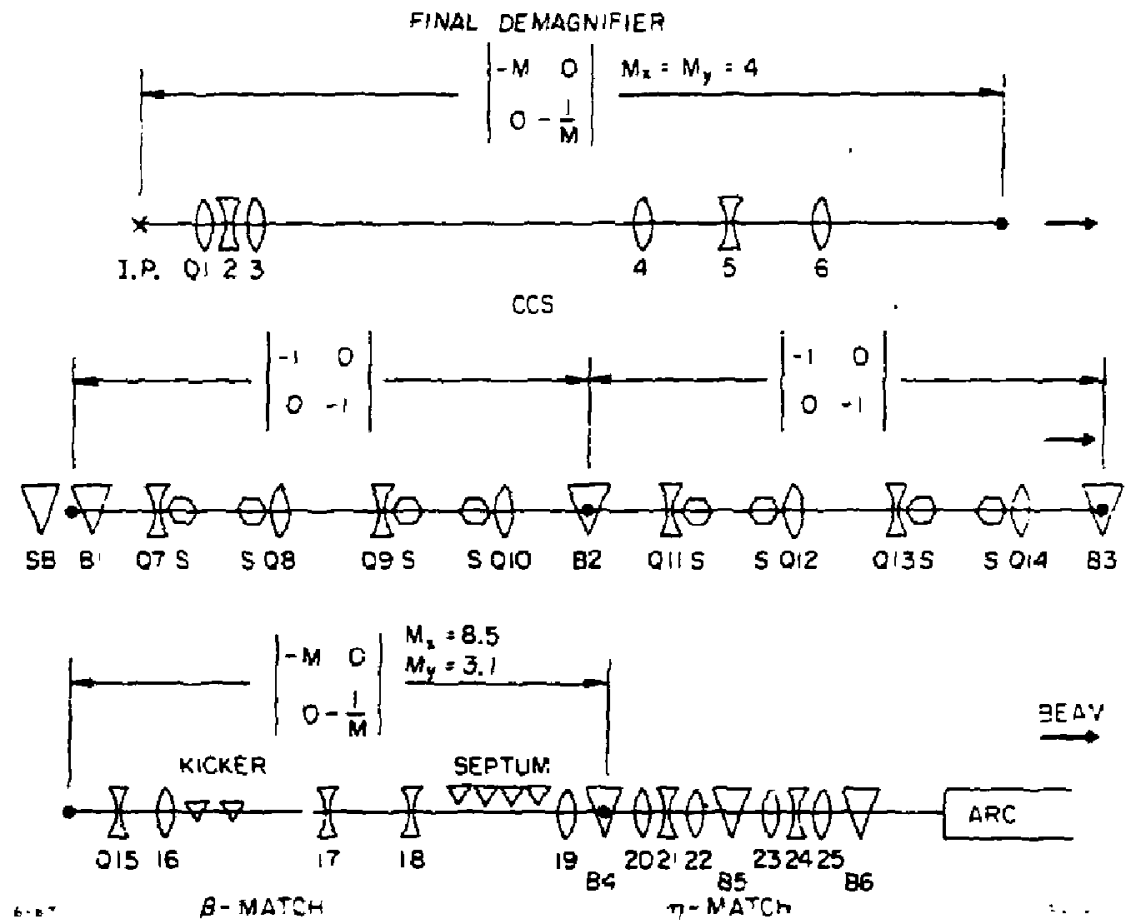

Fis. 11. Schematic of the Find Focus Systen.

\section{Variable Matchine [ad]}

Both the rolume and ahape of the lopol phasepace can be perturbed by imperfections. Erron zenernted within the Are are in principle stable, but the linac is more varioble as the enercy profie

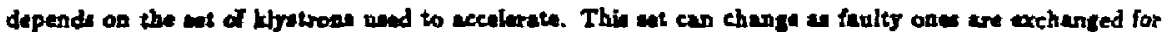

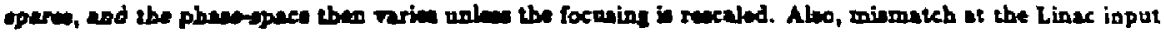
Glemeat throuph the chromaticity of the tinec lattice, if und wbes the enercy represd is minimized at the end but aot bealy. Cood metching in the ${ }^{\top}$ inec it apecielly important at high current, when large local

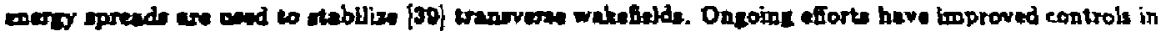

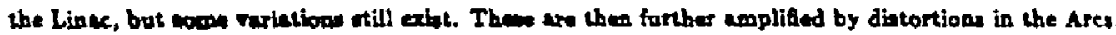

The Find Focts include adjustable matchint minly for static arror accusculated apatreatn. In

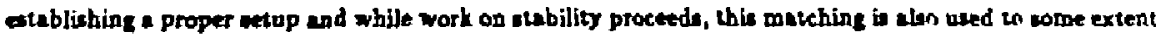
as an overall variable feadbeck. This is not optimal for several reaeons, as will be explained. 
Enhanced amittance or enerw spread are uncorrectable. Larger $\epsilon_{z, y}$ are ajo: concert. for buth luminosity and detector backprounds. The purcly optical dange is slightly worse than linear because of

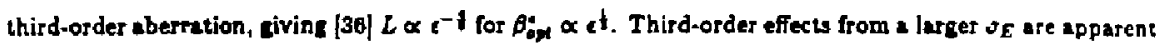
in fog. 10, showing elose to linear loss with $\sigma E$ and weak dependance on $\beta$. The tolerance on $\sigma_{E}$ is about 0.005 .

Optical dislortion is the Arts are most]y linear [40] and are correctable within some bounds. The primary wet enhunces $\sigma^{\prime}$ by correlatinn position with angles or with $\delta_{5}$. amounting to exially offtatting the wairte, $x-y$ couplins $\left(x \phi\right.$ or $y /$ terms) or nomalous $n_{x, y}$. The waist must be corrected to within the depth of field $\beta^{\prime}=0.5 \mathrm{~cm} ; n$, mut be imaler then $1 \mathrm{~mm}$. $A$ acond et enlurges $\sigma^{\circ}$ by perturbing

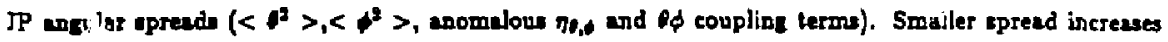
$\beta^{\prime}$, leading to $\mathcal{L} \times 1 / \beta^{\circ}$ from linear optica. Lerger spread reduce $\beta^{\circ}$, but wlso enhances higher order contributions, lading to rapid loss. From fig. $10, \beta^{*}$ must be within $\pm 50 \%$.

Optical cornections: In the deagn $e_{*}=c_{y}$ case, the betatron pharepace can [36] be perturbed is only sx independent ways. With the four disperions, $\eta_{2}, d_{3}, *$, there are thus ten independent distortjons in total. We represent them by those for which tolerances were given: the five IP angular aizes

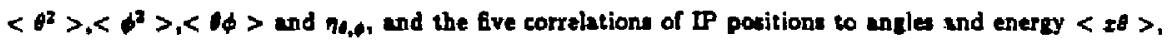

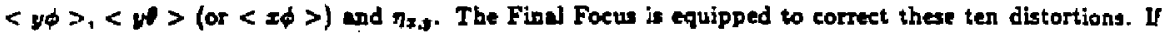
$t_{a} \neq t_{y}$, the ix betatron rariables chomen do not fully describe [41] the phute-sace and more correction elements wre needed.

Dispersion is corrected in the $n$-match with foor quads [13], ingtal]ed in pairs $\pi / 2$ apurt, to control spatial and angular terma, reapectively. Each pair consists of an erect and a stew quad to correct both planes. Naturally orthoconal for amell input error, these correctors are coupled if it is large. Correction range is minly limited by quad strengths, but some values of the input make the correction singalas; for example, when $\eta_{i, p}^{\text {andow }}$

Correctors for betatron minmatch straddle the CCS. The three angular terms are adjusted upstream of the CCS with two arect and one akew quad. The three waist termn cannot be adjusted there independent/y of the angular terms. Theg are taken out with trims on two of the final quads and a second akew quad.

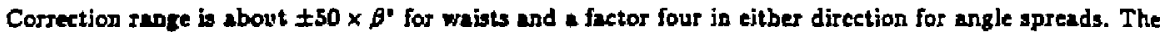
wheme is shown in fic. 12.

Optically, the urtem en correct a large a factor three mismatches. Lo practice, large distortions are not handied well for two reasons.

The firt in lect of arthogonality in the corrections resulting froto aevere opaed limitations. The three waint correction enn internally be made orthogonal [42], but all the others are coupled. This is the care for the two stew quadrapoles, for the extupoles, which muse be refitted after betatron matching as the correctors straddle them, and for the extraction, which atraddle two of the variable lenses and must be reoptimied fiter betatron matchine. The $\eta$-correction is ato non-orthogonai to the $\theta$-match, paricularly [43] for manified or demenified optica] configurations. Operationally, modest variations ate amplified by diatortione remining in the Ares, and car require extensive reoptimization. Efion is theiefore directed both tomards improving the Are lettice and towards correcting variations in the Linuc, to avoid feeding back on them in the Final Focus. 


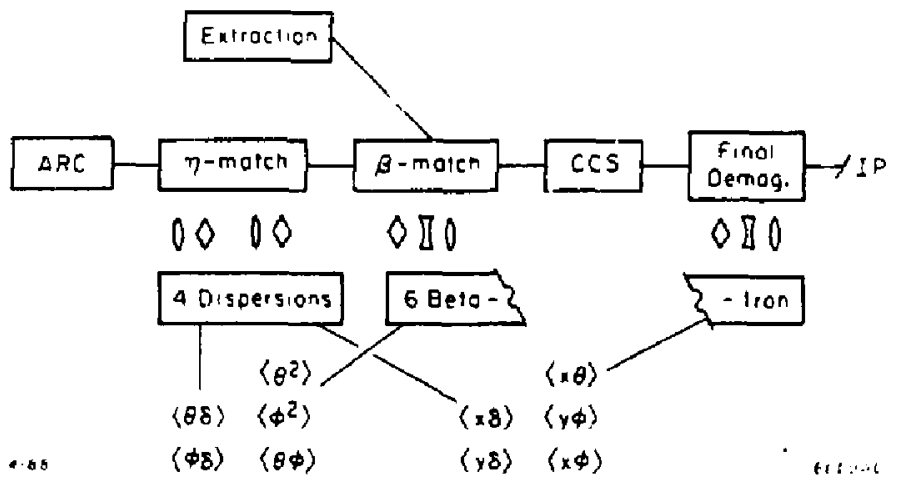

Fig. 12. Optieal matchiog in the Final Focus System.

The second reason is che bartaround induced in the detector by beam-talls impinging on tight apertures at the injection wo the Find Foeus. These aperture as well w some adjustable collimators are upatrean of the main matching elements. Barmful background from hits on these apertures can result from otherwise corsectuble distortions. Recept experimental work [44] indicates more stribgent tolerunces on input errors from this viewpint. This may require introducins further opt ieal corrections in the Ases, and redeployment of sume collimatore aputreum.

\section{Taning Stratecy and Initial Result:}

In this nection, we describe aingle-betm optict] adjutments in the Find Focus. Combined adjustments of severa SLC systems to produce and meintein luminosity with tolernble backfround are ongoing and not described here. Similarly, two-beam tuning and atsering methods are dexcribed elowhere $[45,46]$. Some initial beam lesth are reported.

Wt Grtt mateb [47] $\eta$ from the Arc. Strip-lipe beam ponition monitors are uned to messure beam motion vereus energy. Thin does not give position-energy correlations in the bunch if anomalous $\eta$ exists where the energy in vatied, but give in good eatimate if the Arc in (a expected) the dominast contributor.

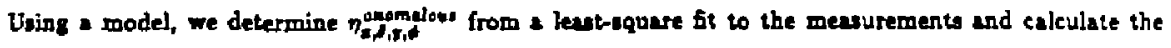
correction. An example of this is shown in fic. 13.

Trajectory erron within the Final Foeut ean abo generate in at the IP. Correction is achieved by undoing mome of the upatream match, although this abo effects [43| waist-corsections and must be the reault of a combined bt.

Betatron minmatch is beat diagnowed new the IP, where angalas and spatial sixes are gaturally oeparated. The three angular terme, $\left\langle\theta^{2}\right\rangle,\left\langle\phi^{2}\right\rangle$ and $\langle\phi\rangle$, are fint edjusted crudely looking at a nominully round spot on s hieh-s ocreen upotream of the Final Triplet. An example of thin is nhown in fig. 14, where the action of the upper stew quad is seen and where the beam ba clowe to the design size.

This does not give $\beta^{*}$ if $t$ is unknown. Als, if weist oftects use large, design size at the high- $\beta$ acreen does not corselate well witt IP angular size. For bether determination, apot aizen are measured at the IP, by scanning the beam acron a thin $5 \mu$ m secondury emission wire target [48], a functions of the three waist 


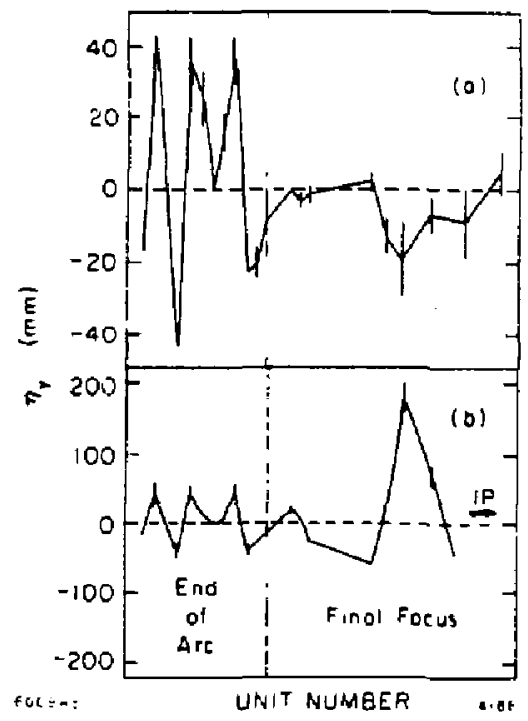

Fis. 13. On-line matehisg of the vertical dispersicn function at the injection to the Final Focus. (b) shows miamatch before correction. (a) shows the effect of the correction. Please note the change of acrle.

controls, starting with the recond ekew quad. Neglecting changes in IP angular spread, $\beta$ - and $\epsilon$ are found fitting $\epsilon \theta^{\circ}+\frac{1}{p^{\prime}}$ to the equare of the bem size. Angular apread are well determined from the parabolas brancher, but both 6 and $\theta^{\prime}$ extimates suffer if the linear spot is not resolved. This arises through residual cross-coupling, only partially correctable for $c_{2} ; 2, y$, and through third-order aberration present before fully matching the $\beta$-function. Sextupoles must also be fitted in the perturbed lattice before scanning the waists, to maintsin good second-order correction. Measured $t$ and $\beta^{\prime}$ values serve as input for fitting the $\operatorname{six} \beta$-motching quada towerds design phase-space parameters. Both on-line and off-line models $[47,49,50 !$ are uned. An empirical eearch around the calculated $\theta^{*}$ is also planned for optimization.

Relativeb mall apote were cometimes obuined originally by only ccanning the waists. An example of thit is shown in fit. 15, where $5 \mu \mathrm{m}$ spot was obtained, with sextupaies turned off all together. The phese-apace bad to be rather cloce to nominal for this to be possible. As input parameters changed, smal! apots did not alwaye reproduce.

More recent]y, the tolerance to input variations has been widened by running with a $\beta^{*}=3 \mathrm{~cm}$ Iattice, ws a starting gueas of the most probable angular spread costection needed. Also, improvements have been made on stability and matching upstream. Beams with smaller thas $5 \mu \mathrm{m}$ sizes are now selatively easy to seproduce for both electrons and positrons with only the three waist-scans. 

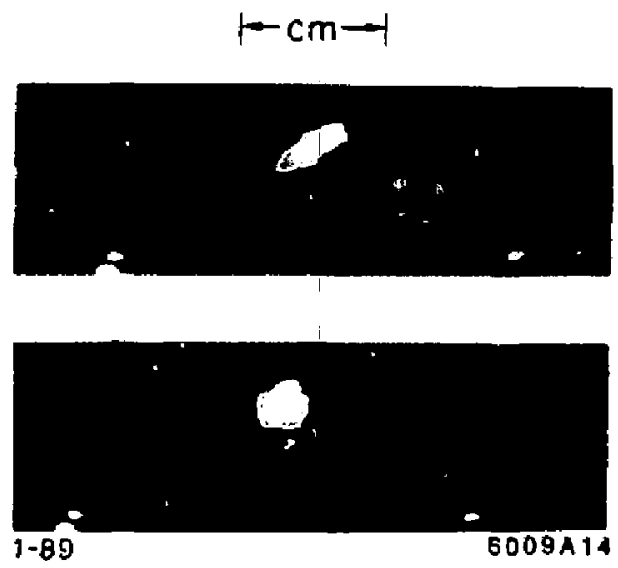

Fie. 14. Crode corsection of crom-coupling in the IP angulas spreade, lookine at the tilt on a sereen at the hich-B poist: (a) before correction; (b) ster correction.

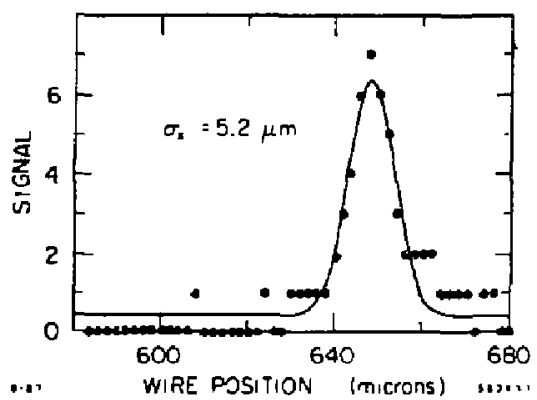

Fig. 15. First measurement of "small apot" at the IP. 


\section{SUMMARY BTATUS AND NEAR FUTURE}

Commimioning is now procesing rapidly towards an initial low luminosity physics run. Both beams are routinely extracied onto their dumpt, and teasonably small heam nizes are usually reproduced at the IP. Main prioritie ane phue-pace controls and ability in the whole machine needed to mainain small spots, background redoctions and zeneral ayatem reliebility and operability. A shift towards operation has been made to unure more continuity in the comminsioning and a more global apptoach cowards stability and cuning iorues. The Mark $\boldsymbol{I}$ detextor, inatlled on the besmline during last year's shutdown, has been turaed on e tew time to help look at beckgrounds. Studies indieate that collimators may have to be redeployed from the Find Focus to upetream places where beams can be comfortably trimmed without zeneratins excenive guons in the detetor. Also, enhencec optical matching in the Ares through horponic corrections is being considered for exly installation, to belp reduce distortions in the Final Focus. Extrapolatiog our recent rate of progreas, and with some well-deserved luck, we hope to produce and detect $=$ hundred $Z^{0}$ before Summer 1988.

\section{ACKNOWLEDGEMENTS}

I have profiled interacting with numerou SLC and Mark If colleagued on several topics described here. New insight and proprea have aften realted. Diacussions with Kar] Brown, Dave Burke, Roger Erickson, Ted Fieguth, Bill Ford, Andrew Hution, Witold Kazaneeki, Joe Mursoy, Nan Phinney, Dave Ritson, Lenny Rirkin, Mure Ross, Matt Sunds, John Seeman, John Sheppard, Ree Stiening, Nobu Toge and many others, were both stimulating and intereating. I have in particular enjoyed working with Karl Brown and David Ritson on optica problems in the Ares and Final Focus. Dave and I collaboraced on the tuning strategy for the Final Focw. I expeciulb wish to acknowledge his contribution. At last, I wish to thank Karl for reading this paper and for aucerting ceveral improvements.

\section{REFERENCIS}

(1) SLC Coneeptual Design Report, SLAC-Report-229, Jure 1980; SLC Deaign Hordbook, Detember 1084; Proeedings of the SLC Wortshop, SLAC-Report-247.

[2] B. Richter, Nucl. Init. Meth. 120, 47 (1976).

[3] B. Richter and R. Stiening, The SLAC Linear Collider-A Status Repart, Proceedings of the Internofional Symparium on Leptent and Phoion Interaction at Bigh Energe (Hamburg, Germany, July 1987).

(4) R. Btienins, Proposed Parameter Specifitation for FY88, April 1988.

(5) J. C. Shepphard et al., Comminuioning the SLC Injector, Proceedingr of the Particle Acceleratar Conference (Washington, D. C., March 1087).

[6] G. E. Fiechet et al, A 1.2 GeV Damping Ring Complex for the Stanford Linear Collider, Proceedings of the Twelfth Intemational Conference on High Energy Aceelerators, FNAL, 37 (1983).

17] J. T. Seeman et d., Experimedta' Beam Dynamics in the SLC Linac, Procedings of the Particit Aecelerator Conference (Washington, D. C., March 1987). 
16\} F. Bulos et al., Deaign of a Figh Yield Poritron Suturce, IEEE Trans. Nucl. Sci. NS-32, 1832 (1965).

|9'j S. Ecklund, The Stanford Linear Collider Positron Source, Proetedings of the Workahop on Intense Positron deams (Idaho Falls, Jdaho, Jure 1087).

(10) G. E. Fischer et al., Some Experiences from the Commissioning Program of the SLC Ajc Transport Syatem, Proceedings of the Particle Accelerator Confercnce (Washington, D. C., March 1987).

[11] R. Erickwon, Find Focus Systems for Linear Colliders, Proctedings of the U. S. Summer Setool on High Energy Particle Aceelerators (Batevia, Illinois, August 1984).

(12) K. Brown, A Conceptual Deigh of Final Focue Syatems for Linens Coljiders, Procecdings of the U. S.-CERN Joint Topical Courec: Frontiers of Partiele Beams (South Padre Llend, Texa, October 1996).

[13] J. J. Murray et al., The Completed Dewign of the SLC Final Focus Syatern, Procedings of the Porticle Accelerator Conferenee (Washington, D. C., March 1087).

[14] R. Stiening. Statu of the SLAC Lines Collider, Procedingr of the Particie Acceleralor Conference (Washington, D, C., March 2987).

[15] J. T. Sezman and J. C. Shepphard, Status of the SLC, Proceedings of the Workshop an New Devel. opments in Pardiele Aecelerator Teehniques, (Orany, Frunce, June 1997).

(16) A. J. Lantford, The Stutu of the SLAC Linear Collides and of the Mark II Detector, Proctedingo of the Severth International Conferenee on Phyrice in Calinian (Tsukubs, Japan, Ausust 1987).

[17] W. Kosnecki, Prowes Report on the SLAC Lineur Collider, Proetedinga of the International Esrophyries Conferenee on Eigh Snerpy Phyries (Uppidl. Sweden, June 1087).

[18] R. D. Ruth, The Statw of the SLC, Procedinge of the ICFA Seminar an Future Peropectives in High Enerov Phyuics (Uplon, Niew York, October 1087).

[19] M. A. Allen et al. Performance of the SLAC Linenr Collider Klyatrons, Proceedings of the Particle Aecelerator Conference (Wahington, D. C., March 1087).

[20] S. Kheifets at a., Beam Optical Deriten and Studien of the SLC Arcs, Proeectingt of the Particle Aecelerator Conference (Wuhington, D. C., March 1987).

[22] M. Sand, The Phyrics of Electron Storage Ringe-An Introduction, SLAC-121 Addendum (1979).

[22] M. Sunds, Emittance Growth from Radiation Fluetuations, SLAC-AP-4T (1985),

|23) R. B. Belm and Z. Wiedemend, Emittance in FODO-Cell Lattice, PEP-Note-303 (1979).

[24] R. Stiening, Dynamie Beam Loading Compentation and Energy Spread in the SLC, SLAC-CN-110 (I981).

[25! K. L. Brown, A Second-Order Magnetic Optical Achromat, SLAC-PUB-2257 (1979).

[26] B. Wens th 2., SLC Are Trumport Syatem-AG-Magnet Measurement and Performance, Proeedings of the Particle Aecelerator Conference (Vancouver, B. C., Cans,da, May 1987).

[27! M. Sands, Betatron Ducillations ond Rolled Achromats, SLAC CN-355 (1987).

28: K. L. Brown et al, SLAC-91, Rev. 2, May 1977. 
(29) D. Ritson. provete communicatirn. June 19:97.

[30] D. Ritaon. BEAMSOM, private program.

[31] L. Rivkin and M. Sonde, private communieation, November 1987.

|32| N. Toge, private communication, March 1988.

:33) P. Bambade, Harmonic Corrections in the Ares, in preparation.

34) R. Stiening Focussing Errors in the SLC Linae Quadrupole Lattuet, SLAC-CN-161 (1982).

'35] T. H. Fietuth, GIAT Committe Report, October 1985.

36) P. S. Bambade, Beam Dynmics in the SLC Final Focus System, Proceedings of the Particle Accel. eratos Conference (Washington. D. C., March 1987).

37) K. L. Brown, private communication, June 1987.

|38! J. J. Murray and T. Fieguth, MURTLE, privale protrem.

[39] K. Bane, WakeField Effects in a Linear Collider, LEEE Trans. Nucl. Sci. NS-32, 1662 (1985).

[40j K. L. Brown and R. V. Servencbx, GIAT Committee Report, Fobruary d0B6.

:11: L. Rivkin, private communication, October 1987.

[12] P. S. Bambade, GIAT Committee Report, Mureh 1987.

(43) B. Ford, privece communication, April 1009.

[14] D. Burke, privale communication, April 1988.

\{15\} P. S. Bambade and R. Erickeon, Bear-Beam Deflections an Interaction Point Dizenoutic for the SLC, Pracedings of the Linear Aceclerder Conferenee (Stanlord, California, June 1986).

(46| G. Bonvicini et al., Beamstrahluns Monitor for SLC Final Focus Uning Gumma Ray Enetgie, Proceeding of the Linect Actelerator Conference (Stanford, Celifornia, June 1986).

[47\} C. Howkes and P. S. Bumbede, First-Order Optical Matehing in the Final Foews Syatem of the SLAC Linear Collider, in preparation.

[18] C. Field el al. Bigh Resolution Wire-Seanret for Mitron Sire Profile Measurements af the SLC, SLAC-PUB-4005, to be publiahed.

[49] W. Kozenpecti, BLTAMAT, prirate propram.

[50] R. Semranck and B. Ford, Flight Sirulator for the Eind Focus, privete program. 
IX. CONCLUSION 
In this thesis, we have developed, tested and analysed the methods for optical tuning in two sections of the Stanford linear collider: the arcs and the final focus. These methods have enabled to reach a quasi-optimal set-up.

In the arcs, we have proposed and studied a design modification reducing harmful effects from the rolls, introduced in the lattice to enable following the terrain of the SLAC site. This modification has made the system much more tolerant to systematic errors.

We have also proposed and studied a new optical tuning method for the arcs, consisting of introducing focusing perturbations at the most efficient harmonics of the betatron frequency. This method has allowed, on the one hand, to make the optical transfer close to perfect, and on the other hand, to adjust the beam empirically at the entrance to the final focus section, to minimize backgrounds produced by mismatches in the phase-space.

In the final focus section, we have designed the optical correction and optimization algorithm. This algorithm has enabled beams focused at the interaction point with three to five micron transverse sizes to be attained, and the optical factors limiting the luminosity to be identified. These factors are mainly the requirement to operate with a $\beta^{*}$-parameter larger than the theoretical optimum, by a factor of five to ten, resulting from the background genersted by beam-tails in the last quadrupoles. 
APPENDIX: REPORTS AND PUBLICATIONS ON THE BEAM-BEAM DEFLECTION DIAGNOSTIC METHOD 
A.1 "Beam-Beam Deflections to Measure Spot Size and

Offset at the SLC IP"

This collider note contains the original proposal and description of the beam-beam deflection method. This method allows to detect the interaction of the beams when they interact at the collision point. It is presently the primary method used to optimize the beams in collision. 


\section{AUTHOR: P. Bambade DATE: June 10, 1985 \\ TITLE: BEAM-BEAM DEFLECTIONS TO MEASURE SIZE SPOT AND OFFSET AT SLC IP}

\section{PART 1: CALCULATIONS}

As sonn as two SLC beams make it to the $\mathrm{IK}$, both transverse offsets, spct sizes and shapes can be extracted from the pattern of angular delections produced by the electromagnetic interaction of the two beams, as one is scanned across the other. These deflections, measured in two high resolution Beam Position Monitors (BPM) mounted symmetrically on both sides of the IP (Fig.1), will produce detectable signais a!lowing spot sizes to be tuned, even witin the very low luminosities expected at turn on. They will also furnish a good signal to monitor beam centering and will therefore become an important part of the FFS feedback system.

This note summarizes the formulae which will allow us to correlate BPM offset readings with the properties of the two beami, and describes the range and linitations of the technique in the case of SLC.

$\bar{F}$ urther work needed includes simulations, specifications for the feedback system, such as its algorithm, veto conditions and so on, aswell as integration of the beamstrahlung signal into the diagnostic scheme.

\section{Basic Principle}

The angular deflection produced by the interaction of a SLC beam with the electromagnetic fie!d of its colliaing partner (see fig. 1) is easily expressed in the simplified case of a round target beam and a point size probe

$$
\theta(\Delta)=\frac{-2 r_{e} N_{I}}{\tau} \frac{1-\exp \left[\frac{-\Delta^{2}}{3 \sigma_{T}^{\frac{2}{T}}}\right]}{\Delta}
$$

where $r_{e}$ is the classical radius of the electron, $\gamma$ the relativistic factor, $N_{T}$ the number of particles in the target, $\sigma_{T}$ its RMS transverse size, anj $\Delta$ the impact parameter of the probing charge.

Deflection versus impact parameter is shown in fig. 2 for targets with 2.5 and $10 \mu \mathrm{m}$ 
transverse spot sizes respectively.

The basic principle of the method is three-fold:

1. Initial beam finding: One beam - the probe in this case - is temporarily suppressed and BPM readings are compared before and after. In the case of initially large impact parameters. the magnitude of the difference is inversely proportional to the transverse offset at collision point and its sign tells in which direction to steer.

2. Beam centering: Scanning the probe across the target and recorcing a plot similar to fig. 2 will allow optimal centering of the two, by looking for the zero defiection symmetry point.

3. Spot size tuning: Since the slope of the deflection at the above mentionned symmetry point is inversely proportional to the transverse cross-section of the target, the same measured plot will allow spot sizes to be infered and thus minimized.

In all three cases, the sensitivity of the method is based on the fact that relative rather than absolute position information is used.

Of course, the simplifying assumption of round target and point size probe is not likely to be satisfied, and the problem therefore needs to be parametrized in two dimensions.

\section{Formulae}

The following results are valid for collisions between gaustian, un- pinched beams.

\section{a. Deflection of point charge}

The deflection of a point charge colliding with a target consiating of a two dimensional gaussian charge distribution can be written $(1,2)$

$$
\theta_{x, y}=\frac{-2 r_{e} N_{T}}{\gamma} \Delta_{x, y} \int_{0}^{\infty} \frac{\exp \left[-\left(\frac{\Delta_{T}^{2}}{2 \sigma_{T_{z}}^{2}+t}+\frac{\Delta_{y}^{2}}{2 \sigma_{T_{x}}^{2}+t}\right)\right]}{\left.\left(2 \sigma_{T_{x, y}}^{2}+t\right)^{3 / 2}\left(2 \sigma_{T_{y, z}^{2}}^{2}+t\right)^{1 / 2}\right)} d t
$$

The aspect ratio is defined by $f=\sigma_{T_{y}} / \sigma_{T_{*}}$. For $f=1$, (2) reduces to (1).

The two deflection angles are shown in fig. $3-5$ as a function of impact parameters in both planes, for $N_{T}=5 \cdot 10^{10}$ particles. Figures $3 . a, b$ and $4, a, b$ correspond to round beams $(f=1)$ with $\sigma_{T_{1.4}}=2.5$ and $7.5 \mu \mathrm{m}$ respcctively, whereas $6.5 .5 . a, b$ correspond to a tlat target with $\sigma_{T x}=25.0 \mu \mathrm{m}$ and $\sigma_{T_{y}}=2.5 \mu \mathrm{m}(f=0.1)$. Note the change in vertical scale going from fig. 3 and 4 to 5 . 
In the limit of large impact parameters, both target and probe are well apptoxi. mated by point charges and (2) reduces to

$$
\theta_{x, y}=\frac{-4 r_{e} N_{T}}{\gamma} \quad \frac{\Delta_{x, y}}{\Delta_{z}^{2}+\Delta_{y}^{2}}
$$

Turning off one of the two beams and comparing BPM readings before and after then allows, via (3), to measure initial offsets.

Taking the limit for small impact parameters gives the linear dependance

$$
\theta_{z, y} \simeq \frac{-2 r_{e} N_{T}}{\gamma} \quad \frac{\Delta_{x, y}}{\sigma_{T_{x, y}}\left(\sigma_{T_{x}}+\sigma T_{y}\right)}
$$

Measurement of the slopes of the deflections as a function of impact parameters will then allow, via (4), to infer the two transverse sizes of the target beam to be tuned.

b. Form factor accounting for finite probe beam size

When the two beams are close, the average defiection measured in the BPM is reduced due to the finite size of the probe. This can be accounted for via the convolution of the probe density distribution with (2). The result of the calculation is summarized in two-dimensional form factors, by which the slopes in (4) are reduced, and given by

$$
\begin{aligned}
& F_{x}\left(f, R_{x}, R_{y}\right)=F_{y}\left(1 / f, R_{y}, R_{z}\right)= \\
& \frac{1+f}{2} \int_{0}^{\infty} \frac{d t}{(1+t)\left[1+R_{z}^{2}+t\right]^{1 / 2}\left[f^{2}\left(1+R_{y}^{2}\right)+t\right]^{1 / 2}}
\end{aligned}
$$

where $R_{x}$ and $R_{y}$ are the ratios of probe to target transverse sizes in botí planes $\left(R_{x, y}=\sigma_{P_{x, y}} / \sigma_{T_{x, y}}\right)$. As is consistent, the form factors become one for small $R_{x, y}$ and zero for large $R_{z, y}$. The functions are shown in fig- 6 and 7 for target aspect ratios $f=.1$ and $f=1$.

In the special and initially unlikely case of round target and probe beams $\left(R_{x}=\right.$ $\left.R_{y}=R, f=1\right)$, (5) reduces to

$$
F_{\text {Round }}(R)=\frac{\log \left(1+R^{2}\right)}{R^{2}}
$$

The corresponding plot is shown in fig. 8 . 


\section{Application to SLC : Numbers and Ranges of Utilization}

\section{a. Limitations}

Three types of limitations are anticipated. The main and most obvious one is the performance of the measuring BPMs. The better their resolution, the smaller the measurable offsets and the more effective the method. We hope for $20 \mu \mathrm{m}$ or better on a single pulse. Particular attention has to be payed to their directivity, sisce beams will be measured from both directions. Harmfol synchrotron radiation present in the environmant will furthermore have to be masked.

The second limitation, the stability of the beams, is a fundamental limitation of SLC and is common to all colliding beam diagnostic and tuning procedures.

A third difficuly arises from the non-gaussian, assymmetric distributions expected as a result of Linac wakefields and Arc non-linearities. Beam finding at large impact parameters is not affected, but the pattern of deflections shown in fig. 2 and 3 will be distorted to the extent that a lot of charge is carried by the tail. This and uncorrelated tilted $x-y$ distributions of the two beams, due to errors, will make the patterns hard to interpret. Fortunately, tuning for maximum slopes will always lead us in the right direction in the same way as beamstrahlung can be tuned for maximum emission power.

\section{b. Scaling laws and relation to luminosity}

The scaling laws are more favorable at low luminosities for this technique than for other beam-beam related signals.

The deflection at large impact parameters, which will be used for initial beam Ending, scales as $N_{T} / \Delta_{\text {, as }}$ can be seen fiom (3).

The maximum deflection produced as we scan the beams across each other to minimize spot sizes scales as $N_{T} / \sigma_{T}$, or as the root of luminosity

\section{c. Beam finding and centering}

Initially, a first SLC beam will be threaded, at low repetition rate and intensity, through Arc and FFS, and to its dump. After best possible optimization of transmission and optical parameters, the above operation will be repeated with the other beam. BPMs on both sides will then be used to match the two orbit as well as possible. After this is done, the two beams will miss each other at the IP by offsets of a fiw hundred microns, which accounts for linited absolute BPM accuracy and alignment errors.

Bringing the two beams into collision, in preparation for spot size measuremen. 
will have to be done at full intensity since upstream wakefields, which affect the pirameters to be tuned, are intensity dependant. Turning one of the two beams off momentarily will then produce an offset of

$$
\Delta R^{B P M} \simeq \frac{-2 N_{T}, T_{e}}{\gamma} \frac{1}{\Delta} d_{f P}-\mathrm{BPM}
$$

in the BPM located 2.5 meters away. When this offset is large enough to be resolved, so that we know that the beams feel each other, they can be centered by mapping out two dimensional surfaces similar to those shown in fig. 3 to 5 , in order to find the symmetry points.

Assuming $20 \mu m$ resolution, the range of utilization can be written

$$
\frac{N_{T}\left[5 \cdot 10^{10}\right]}{\Delta[100 \mu \mathrm{m}]} \geq 2.8
$$

This means that at $N_{T}=5 \cdot 10^{10}$, signals will be measurable when the beams pass withir: $350 \mu \mathrm{m}$ of each other.

c. Spot size minimization

The maximum offset produced in the BPMs, during the above described mapping can be approximated by

$$
\left|\Delta R^{B P M}\right|_{\max } \simeq \frac{2 N_{T} r_{C}}{\gamma \sigma T} \cdot F_{R o u n d}(1) \cdot d_{I P} \rightarrow \mathrm{BPM}
$$

or

$$
\left|\Delta R^{B P M}\right|_{\max }[\mu \mathrm{m}]=2000 \frac{N_{T}\left[5 \cdot 10^{10}\right]}{\sigma_{T}[2.5 \mu \mathrm{m}]}
$$

Also here assuming $20 \mu \mathrm{m}$ resolution, the range covered is

$$
\frac{N_{T}\left[5 \cdot i 0^{10}\right]}{\sigma_{T}[2.5 \mu \mathrm{m}]} \geq 10^{-2}
$$

which corresponds to a luminosity of $4 \cdot 10^{25} \mathrm{~cm}^{-2} \mathrm{~s}^{-1}$. 


\section{References}

(1) P. Bambade, These de Doctorat 3eme Cycle, Centre d'Orsay (1984)

(2) For example : S. Kheifets , PetranNote 119 (1976)

\section{Acknowledgements}

Many useful discussions with G. Bonvicini, G. Bowden, R. Erickson, H. DeStaebler and A. Minten are eagerly acknowledged. K. Bane suggested useful tricks for calculating some of the integrals. 


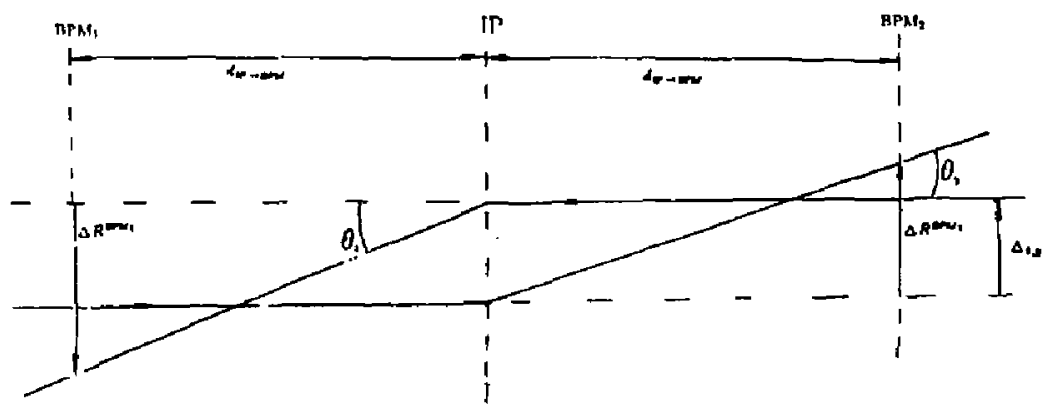

fig. 1

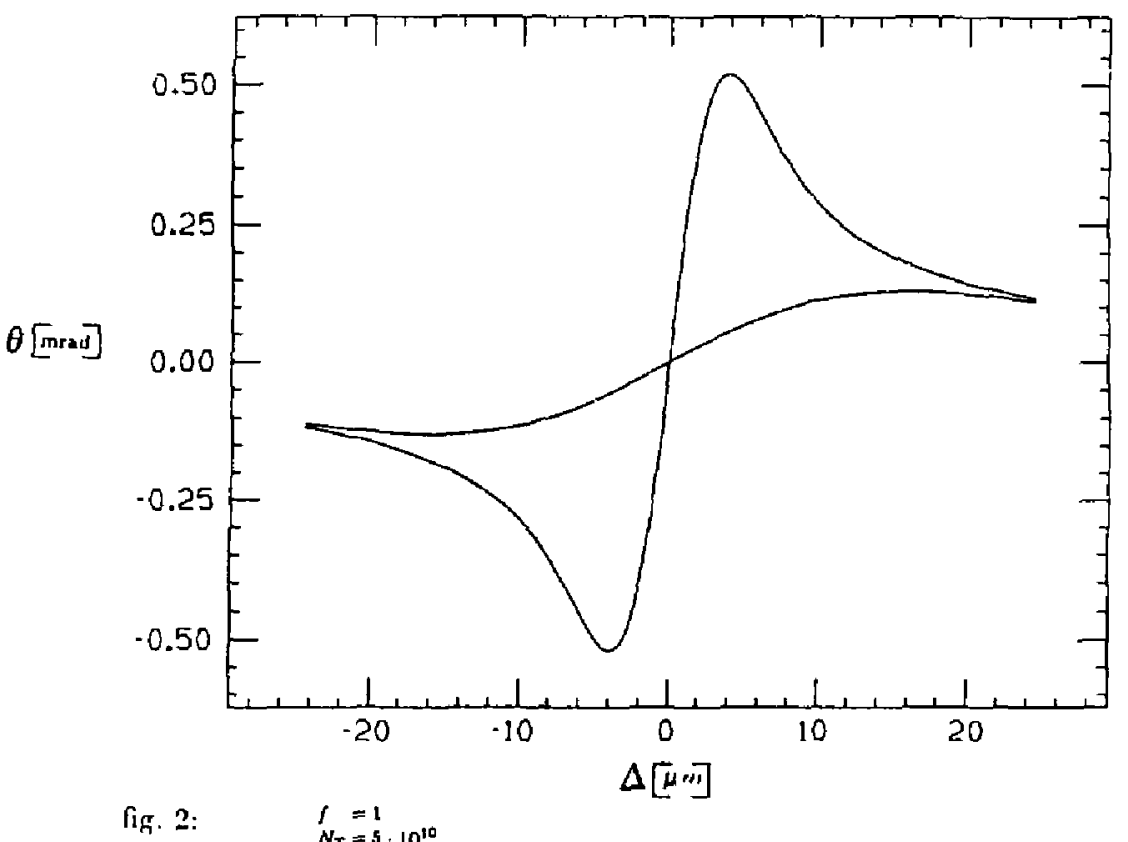

fig. 2: $\quad f_{N_{T}}=5 \cdot 10^{10}$ 


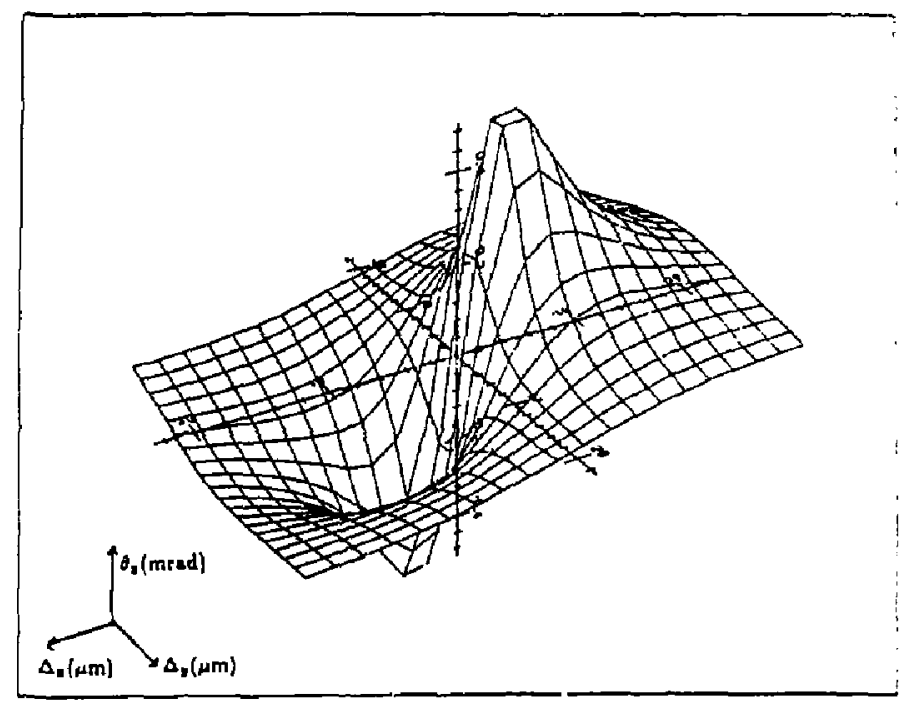

fig. 3.a: $\quad \begin{aligned} \sigma_{7 \mathrm{~s}} & =2.5 \mu \mathrm{mm} \\ N_{T} & =5 \cdot 10^{10}\end{aligned}$

$f=1$

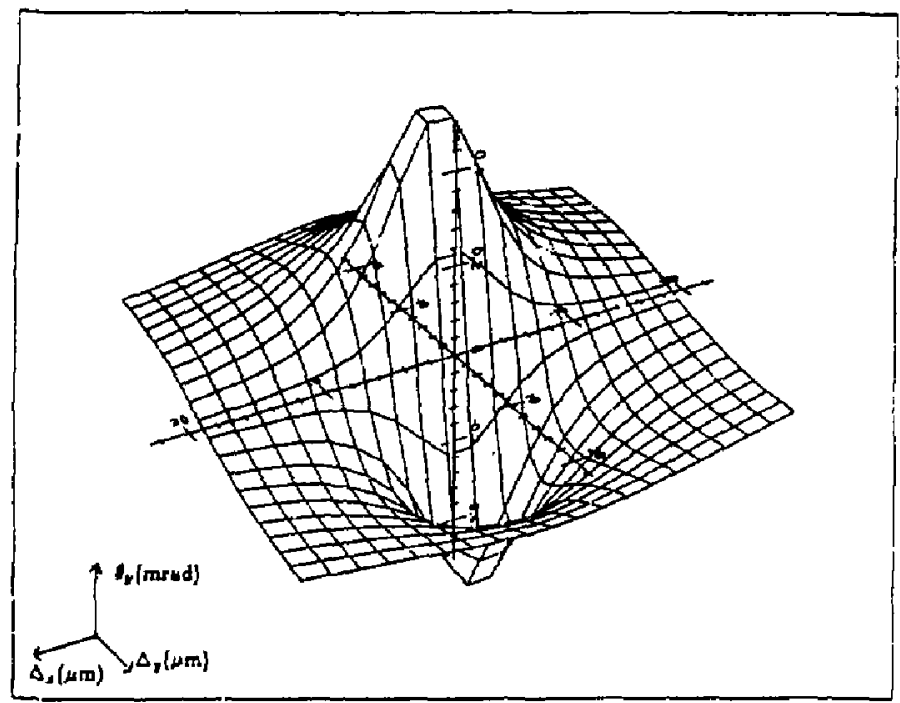

fig. 3.b.:

$$
\begin{aligned}
\sigma_{T_{1}} & =2.5 \mu \mathrm{m} \\
N_{T} & =5 \cdot 10^{10} \\
f & =1
\end{aligned}
$$




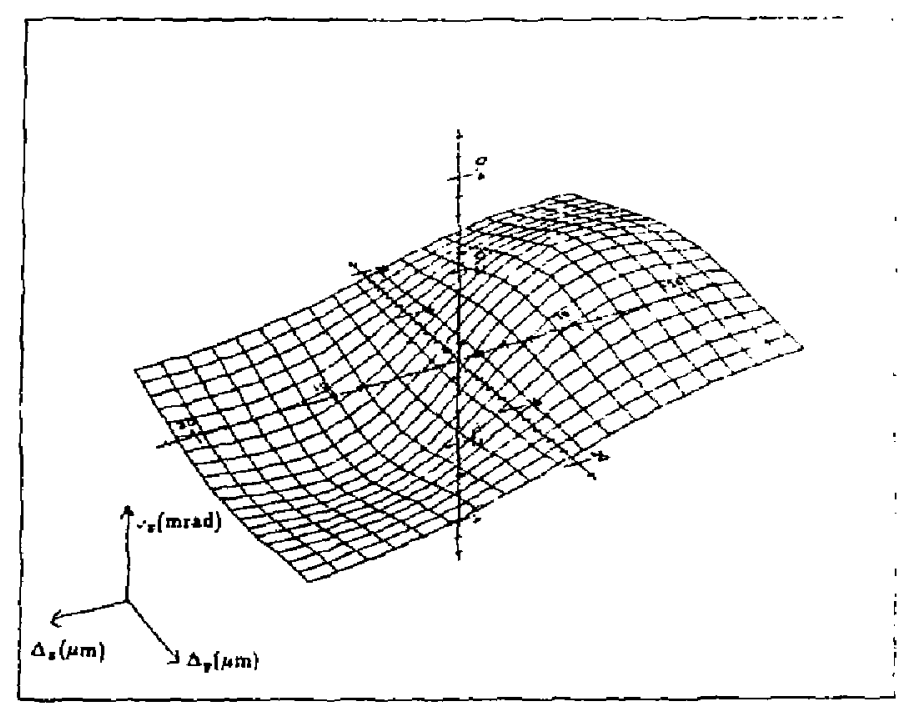

fig. 4.a: $\quad \sigma_{\mathrm{r}}=7.5 \mu \mathrm{m}$

$N_{I}=5 \cdot 10^{20}$

$r=1$

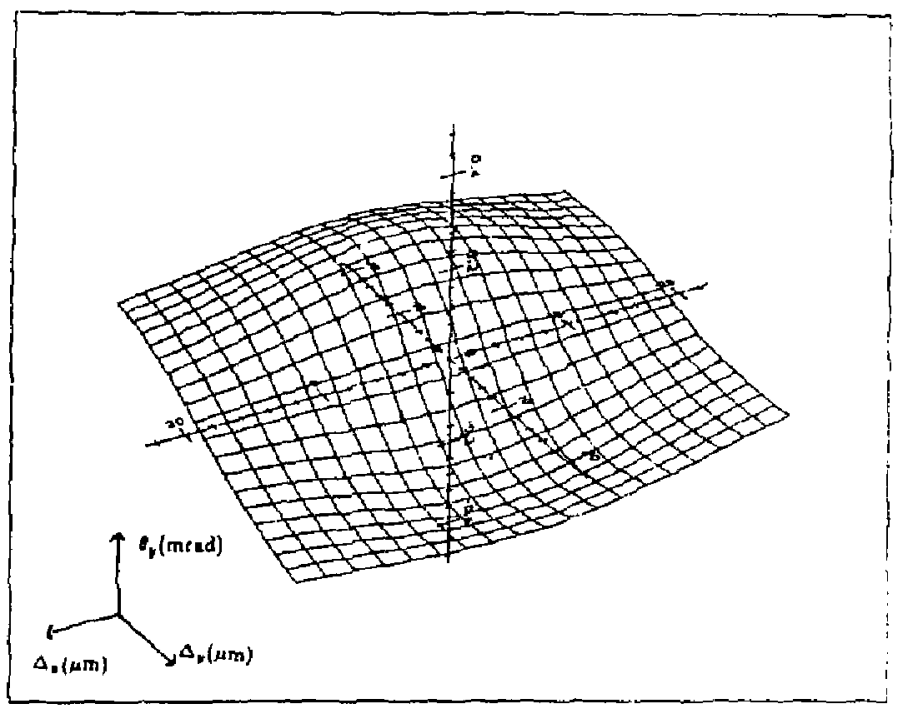

fig. 4.1:

$$
\begin{aligned}
& \sigma_{T_{0}}=7.5 \mu \mathrm{m} \\
& N_{T}=5 \cdot 10^{10} \\
& f=1
\end{aligned}
$$




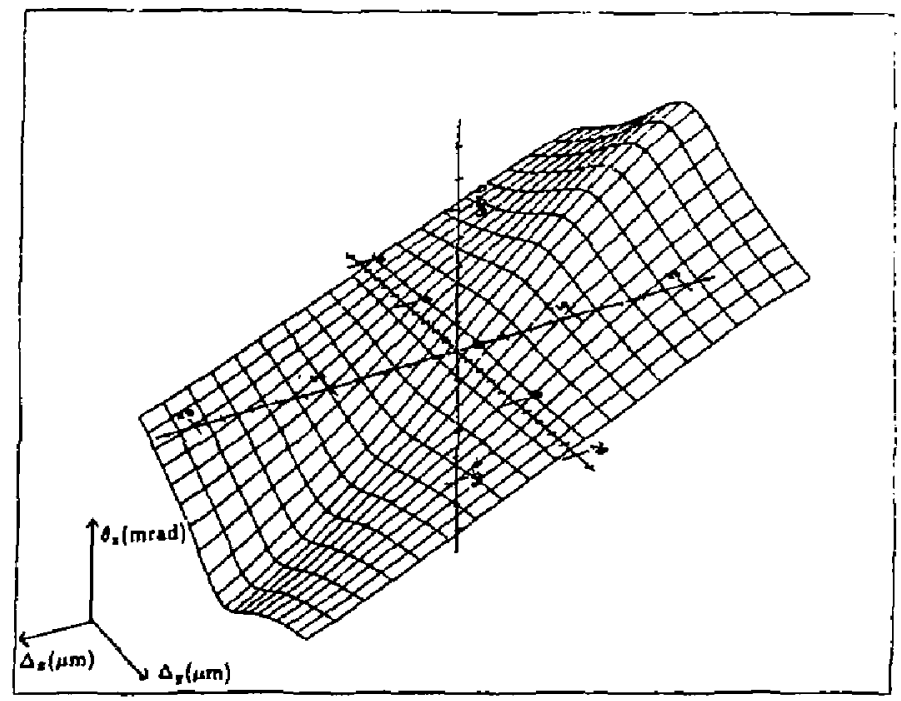

fig. 5.a:

$$
\begin{aligned}
\sigma_{2,} & =25 \mu \mathrm{m} \\
N_{r} & =5 \cdot 10^{10} \\
f & =1 / 10
\end{aligned}
$$

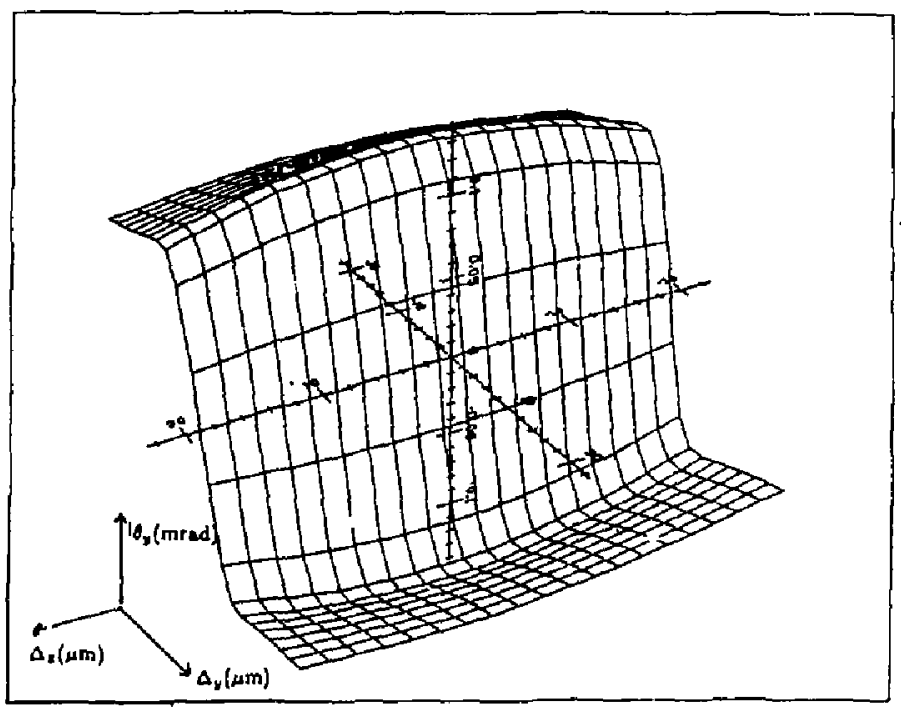

fig. 5.b: $\quad o_{T,}=25 \mu \mathrm{m}$

$$
\begin{aligned}
N_{T} & =5 \cdot 10^{10} \\
F & =1 / 10
\end{aligned}
$$




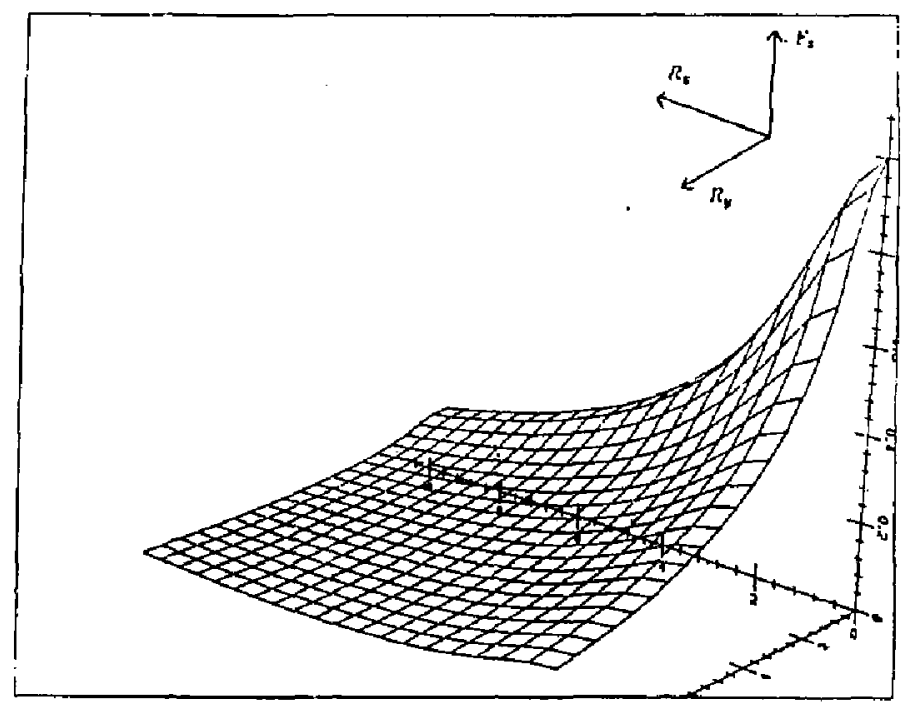

fig. 6: $\quad t=1$

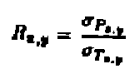

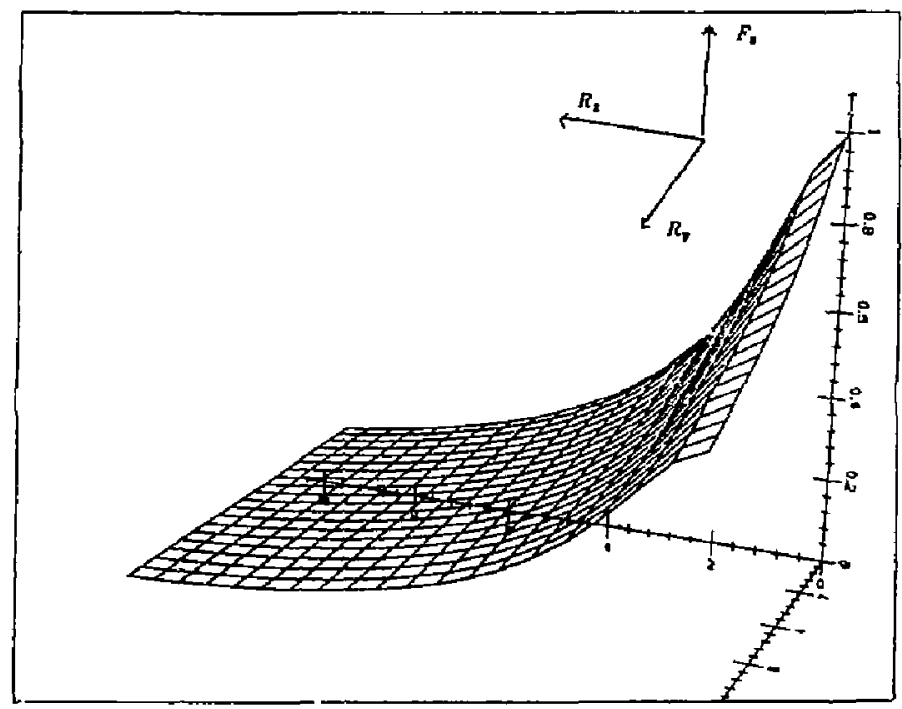

fig. 7 :

$$
\begin{aligned}
f & =1 / 10 \\
R_{z, y} & =\frac{\sigma_{P_{a, k}}}{\sigma T_{T_{,}}}
\end{aligned}
$$




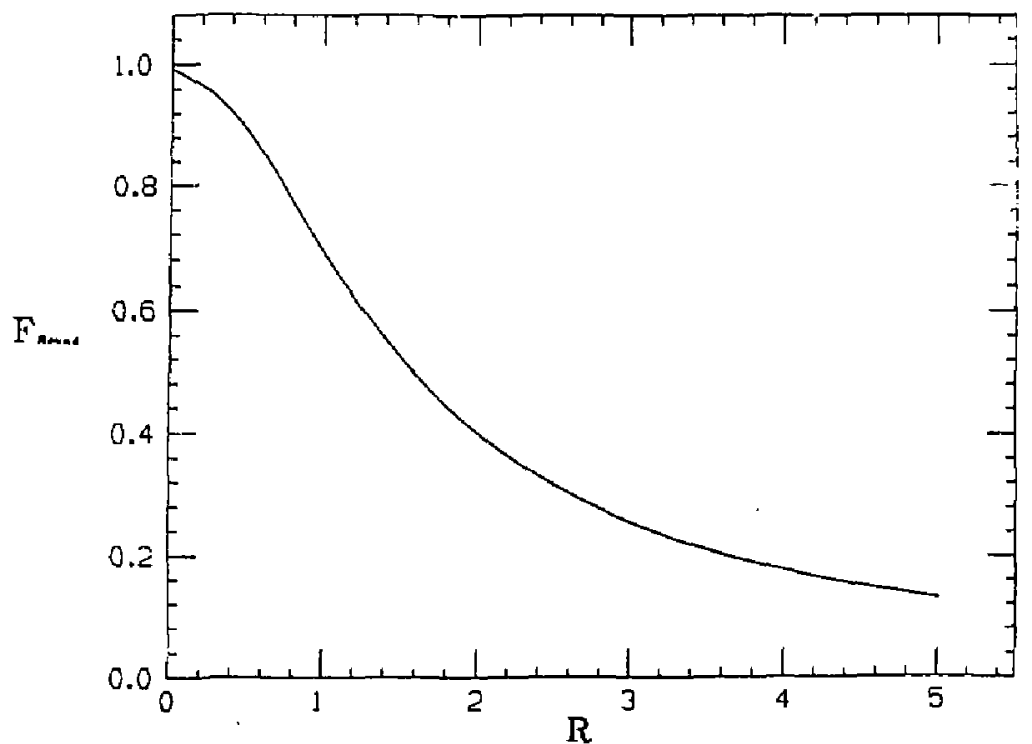

fig. 8: $\quad r=1$

$$
R_{\mathbf{n}}=\boldsymbol{R}+\mathbf{y}+\boldsymbol{R}
$$




\section{A.2 "Beam-Beam Deflections as an Interaction Point Diagnostic for the SLC"}

This conference article gives a more detailed description of the experimental method and an outline of the diagnostic hardware used. 


\title{
BEAM-BEAM DEFLECTIONS AS AN INTERACTION POINT DIAGNOSTIC FOR THE SLC
}

\author{
P. Bambade amd R. Enckon \\ stapond Lines Accolenwer Candur

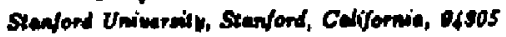

\begin{abstract}
Abotract
A technique is decribed for non-dentruetive mennrement and monitorins of the owering oftian of the electron and ponitmn beams at the intarcetion point of the SLC, band on uing atripline benm-ponition moniton to manure the centroid of

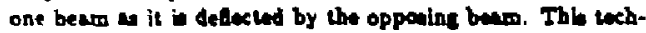
nique is ano expected to provide djopontic injormellon reloted

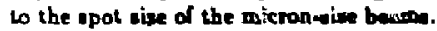

\section{Iniroduction}

The electromesnetic force acting betweed two latene col liding beam of opponitely ehered particles will cause thom to be dedectod in pasine by an ensle that dependy on the of. cet betwen the bunchen, and the dintribution of chersen within the bunches. Thit detection, mencurehle wh aodetructive

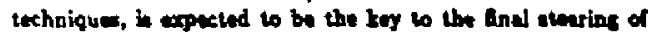
the $e^{+} e^{-}$beams in the SLC. More somerally, the beam.beam dellection phenomenon in a menurable manitutation of the collinion of micron-ive beams and in applicable to an large future linatr collider.

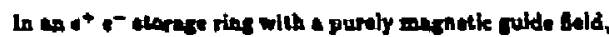

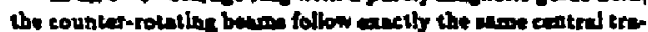

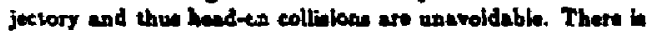
no \& priori rueses why this whould be true if thens colliders,

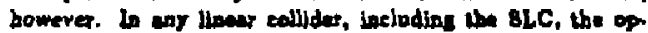

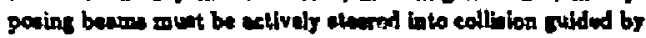

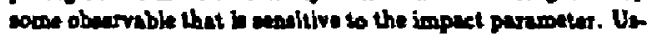
ing state-of-thert utip-line buen poultion monitors (BPMb), it may be pomible to direct the two bane independently in

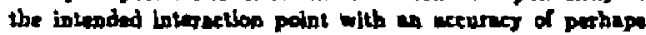
$100 \mathrm{~cm}$. In ondat to echlere accoptable luminosity vith the SLC, the beam anst be terrad to within oboul one beam

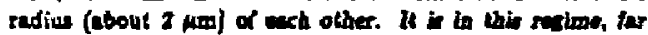

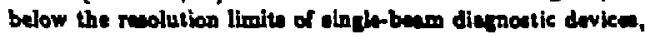
thet ihe benm-bean defection il atrontent.

\section{Danle Formulne}

The anfular delection produead by the intaraction of an SLC basm with the eletromenetic sald of it collidins partaer can be ationed analytically in tha dimplibed cast of two

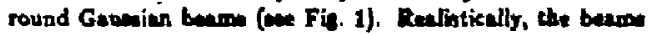
are hol expected to be roond and gaveian ontil the final focu opticul tuning i completed, a procedure that requires that the beame be colliding. A tromdimentional parametrixsion for the collizion of two banp with traneveree distributions af arbitrary faten and arieatetion is gives in Ref. 1.

The detlection of a eingle particle of cherse e, pewing at an offect $\Delta$ from the centroid of an opponitely charged Geuerian distribution, in given by:

- Work oupported by the Dapartmeat of Fnergy. contract DE-ACO3-765F00515.

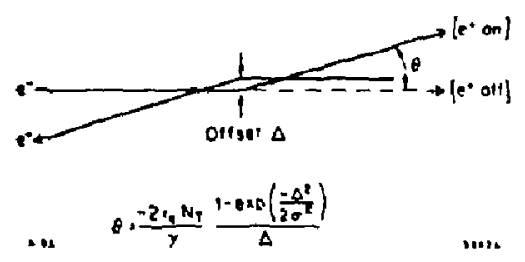

Fin. 1. The trajuctory of acb besm is detected by the oppeaing banm paving at on oliset $\Delta$.

$$
f(\Delta)=\frac{-2 P_{\Delta} N_{T}}{7} \frac{\left.1-\Delta_{0} \mid-\Delta^{2} / 2 \sigma^{1}\right)}{\Delta},
$$

where $r$, is the cilenicel fadiue of the electron, 7 the relutivin the $E / m$ teclox, NT the number of particle and o the RMS traneverw aise of the Ganemian dintribution.

When the beame pan with olvote large eompand to thoir tranvera dise, they we each other a polat charte and (1) b a cood epproximetion for their motual dadection. Whan collidins with = enell ofret, the snith aisen of the beas ditributions muat be taken into cecount. Thit een be done by convolutiog (1) will the diotibutlor of the oppaing beam. The reole of meb a calculation, enrried out in the lionte of

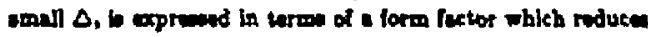
the overage dellection:

$$
F(R)=\frac{\ln \left(1+R^{2}\right)}{R^{2}}
$$

Bre $\boldsymbol{R}$ in the ratio of the tranverse nites of the wo beams.

Dafection varem ofiel is plotted in Fly. 2 for $80 \mathrm{GeV}$ beams consisting of $i \times 10^{10}$ particle, with trenvern apot sices of 2, 5, and $10 \mathrm{wm}$. $10 \mathrm{\mu m}$ in the atterated aive of the beam of the SLC inturetion point bufore apticel cortections ure made. Mront wething tros and minalignmente contribute to thin atimate. By adjuntine the thel focus corrector mes. nets, $\sigma$ cen be ruduced lo about $2 \mu \mathrm{m}$. The ubove farm fuctor ba been lecorporated in the curves at a multiplyint reduction fector, esouming in each case $R=1$.

\section{Deflection Delection}

Several methods bave been atudied for devectinc and metouring the beam-benm deflections. The most obvious is to whe - pair of BPMo atradline the inhection point. If the drift length "lever an" in lons enouch, edelection at the 1.P. will reult in s westureble protion abin at the BPM. The power of this method can be treatly enhenced by euppraning the oppowing benm on come puive and wetchins the menared beam jump betk to it undetlected potition. To mate this poupible, a peir of epecial pulsed mognets, the eingle searo dumpers", 


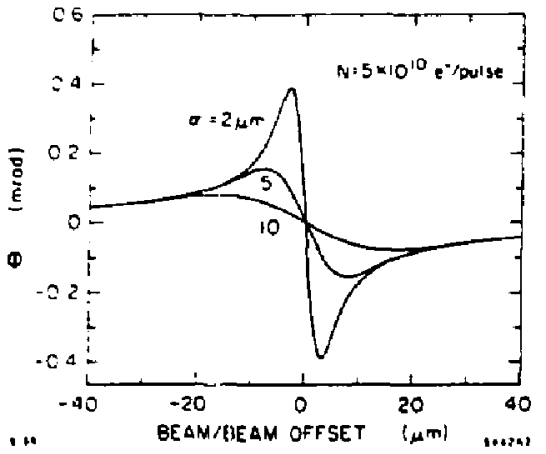

Fir. 2. The deflection angle $\theta \omega$ fusction of oflat $\Delta$, plotied for three opot sizes.

will be provided upetream of the find focus to kick either beam out of the tranport ayotem on commend.

In prifciple, the benm-benm deflection cen aleo be obearved with conventional sereen proble monitnre located in the paths of the outfoing extracted beam a they are tranoported to the dumps. In she SLC, wuch meanurements will be pousible in the rertical dimenion only. Defection in the horiented plane will be obscured by the momentum dimperaion introduced by the extraction exptum monets. As part of a planned uperade for the gorth extrution line, ${ }^{1}$ it will be pauble to cancel the dispersion with additional magnets to enable deflection merurements in both the horisontai and rertical dimention. In any ease, poution meanurements in the extruction lines provide dentially no informution boul the aboolute ponition of either beam near the 1.P., becaive of the laze number of muenets, traverned by the outgoing beam before reaching the extraction line. However, relative ponition shifle ean be orenured unins devices in the extraction line in conjunrtion with the aingle. bearn- "umpers mentioned above to cive weful measure of the defection at the I.P.

Another eppronch is bued on detecting beamatrahlung radiation. This in the name riven to the eyachrotron radiution emitted by each beam $a$ it in deflected by the other. The ensular distribution of this radintion, etroncly peated forward in the direction of the outgoing beam, can be meaured with a suitable detector along a line of witht but quite diatant from the interaction point.

\section{Application to Stecring and Tualng Procedute}

A three-otep tuning procedure in envisioned:

1. Initial bew finding: One beam-denigneved the "turget" in thia cae - is momentarily suppreatd with a ainglebeas dumper while position merurements are mude on the "probe" beam. In this way. the shif induced by the target beam ean be determined. When the ofiset between the beams is larce, the maenitude of the ahift in inverely proportiond to the offert and its tien tell in which direction to oteer. This can be eeen by takin the limit of (1) for larte $\Delta$ :

$$
\varphi(\Delta)=\frac{-2 r_{E} N_{T}}{\gamma} \frac{1}{\Delta} .
$$

2. Beam centering: Scanning the turget acrom the probe and recording a plot timilar to Fis. 2 for the probe will fecilitate optimal stering of the two benms. The serodeflection aymotry point in Fic. I L resched when the beame are purfectly centered.

3. Spot aise tuning: Taking the limit of (1) for wowl $\Delta$ and multiplyine by the form factor (2) tive:

$$
\theta(\Delta)=\frac{-r_{0} N_{T}}{T} \frac{\Delta}{\sigma^{2}} F(R)
$$

The slope of the detection of the probe benm nesr the sero-defection mymetry point in invenely proportion. to the crow-aetional ares of the curget. By diffewntiating (1), it can be wen that the defection in maximum for offate of about 1.6 etendard deviatlons of tive target dintribution, and that the maximuto delletion ecales w the inverse of the tranveres opot tine:

$$
\theta_{\text {met }}=0.451 \frac{2 r_{c} N_{r}}{7} \frac{1}{\sigma} \text {. }
$$

A relative mesure of apol aise can the be obtained by cesnnins one beam acrom the other 4 in SLep 2 bbove. Guided by these menurements, an operator can adjust eptical elements of the trasuport oytem to minimize thit tinel spot oiro.

The procedure ductibed here are baned on rtletive met eurements of the oukging beam pouition at lockllons where the angular detection produced in the collinion leads to a trane v.une poition abilt. Meny of the BPMs in the outzoing urentport aylem have wuituble phese thind from the IP wo be uned for thit purpose. The but location, hewever, are in the final optical trangformer quedrupolea, where the $\rho$-function reach their lurest value, thereby marnifying the defectuon the most, and where dispention in neslitible, (which minimises confunion with enercy vitiotion). Thre BPMn, near quadrupoles 21, 3 and 4, ate planned for thin purpose (Fix, 3), Euch hes a entective optical lever arm of about 3 mater. Position thifto correaponding to a wide range of IP parametert can be tewolved at thene locations.

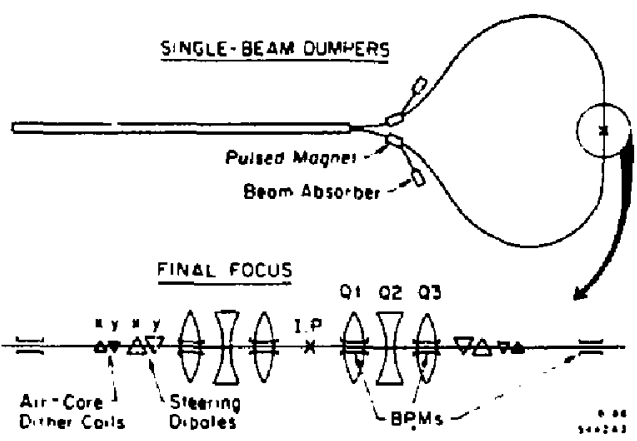

Fic. 3. Schemulic of besuline components relevant to the deflection technique. 
The ueful rance of these techniquen, i.e., the maximum ofiet that still give a meanuruble deflection, in limited only by the ability of the BPM to twolve beat centroid movementa. For exemple, erume the BPM aen $Q 1$ can reolv the entroid panition of a single bunch of $3 \times 10^{6}$ particle to s level of $20 \mathrm{~mm}$. It will then be pouible to detect relutive beard-ben oficte up to a meximum of:

$$
\Delta(\mu \infty)=10 \frac{N_{T}}{5 \times 10^{\circ}}
$$

For larcer bens eurents, in may be poejible to do betlet then the limit indicated in (5), becaue the BPM rmolution also improves with increasing curtent. By chopping one ban of and on wine the tingle berm dumpers and sverating over many pubes, the rasaiation can be improved further. Although maginal al low inkennity, this beam findint vechnique -hould bridge tbe gep between the uaul arbit metchine methods which sely on sbo'vu BPM acuracy to ateer the beams independently wo the IP, and techniques besed on luminosityrelated signals, wch wenstrahlung, disruption imating," and the Bhabha ecstiering rate.

\section{Dynumle Frrort and Corretlave}

It in expected that even when the static crouting error: buve been corrected a dactibed sbove, the two benme will not remain centered on each other without in active faedback system. Many cource of drift and jitter that could eause the beamy to wander at the IP have been identified. In mont eman, these effect can be minimized with careful attention to relevant hardware derigns. Magnet powet supplie, for example, wut be well reculeted, and oupport structores wust be rigid. Natural ambient ground vibrations at frequencies above I H2 have been thown" to be negligible, athough wome local man-made vibration sourcea such w reciprocatins pumpa could couse problems if aot isoleted. On a slower time cedte, thermes effecte will caue mechenical upport atracture to expend, and power aupplies to drift enough to adversely effect the luminos ity unles ateering corrections are made. Studie of tudbuck whemes for the SLC have focured on timple and relatively alow algorithim, althoush the BPM electronic, control ryatem, and other key componsmit are being built to allow pulaeby-pule feedback to accommodate fanter ar more complex schemes.

A imple leedbech aforithm for corsectint relatively alow drifu in besed on eulomstieslly appreaning one beam periodically uning the-kingle beam dumper. Of eaure, the luminoity would be sacrificed on theac occarional pulees, but they would enubie ateering correction to be computed from the measured parition whift of the outgoing beam. Becaue each wetured dafection can corrmpand wo two ponible oflete, the aperation he to be carried out frequently enouth to ensure that the axtud ofiet doen not drift outside the domain of the IP, bounded by the detection maxims, in the time betwen updates. This approaeh in probsbly adequate 10 track the thermal expanaion of eupport etructures and other wachenied effects.

An upproach that does not require acrifieing any beam pules would be to excile omall "dither coil" dipoles (Fis. 3 ) in aproptamened why to induce amill periodic oftech at the IP, with an anplitude of a fruetion of a otendurd deviation. In thin way, one beum can be mude $w$ trace out a patlern weh a amall circle at the $D$. The defectione of the oppoins beam will then project the same pattern at the BPM. When the offaet between the beam corremponds to a point on a ateeply riaing poultive alope in Fig. 2 (beyond the 1.60 pesit on either eide), the projection in marnified image of the dither pattern. When the offet in lese then 1.60 , the projection is an inverted imstre of the dither pattern. Syachronou patition mearurement would then alow a determination of whether the beam were collidint within or beyond 1.6 atendard deviations of each othet. If necentary, a correction could be epplied to bring them buck to within one $\sigma$. The tign of the defection would indieate the direction in which to ther. In both these algorithms, corrections are applied uins steering correcton immediacely upitream of Q3.

\section{Aeknowlodzement:}

We winh to thent the member of the SLC Final Focur Group for helpful dieturtion on thit lopic, and in perticular W. Kozenecti for encouraging un in put this poper wether.

\section{Reforencet}

1. P. Bambade, "Beam-Beam Deflectiona to Menture Spot Size and Offut at SLC IP," SLAC CN-303, 1085.

2. Mark II Collaboration and SLC Find Focus Group. "Extraction-Line Sptctromeleta for SLC Energy Mea. vurement," SLAC-SLC-PROP(2), 1986.

3. G. Bowden, Private Communicution.

4. G. Bonvicini, C. Field and A. Mincen, Benmatrahlune Monitor for SLC Final Focu Uaing Gamma Ray Enerciea," Procedings of this conference.

5. G. Bowden, "Mechunical Vibration of the Final Foeus," SLAC CN-914, 19B5; G.E. Ficher, "Ground Motion and Ito Effecte in Accelerator Deign," SLAC-PUB-3392 Rev., 1985. 
A.3 "Observation of Beam-Beam Deflectious at the Interaction Point of the Stanford Linear Collider"

In this publication, we report the first measurements of beam-beam deflections at the SLC. The successful application of this method to maintain the beams in collision is described, with a summary of the experimental linnitations. 


\title{
OBSERVATION OF BEAM-BEAM DEFLECTIONS AT THE INTERACTION POINT OF THE SLAC LINEAR COLLIDER*
}

P. BAMBade, (1),(a) R. ERICKSON, (1) W. A. Koska, ${ }^{(2)}$ W. Kozanecki, ${ }^{(1)}$ N. PHINNEY(1) AND S. R. WAGNER ${ }^{(3)}$

(1) Stanford Linear Accelerator Center, Stanford University, Stanford, California 94309

(2) University of Michigan, Ann Arbor, Michigan 48109

(3) University of Colorado, Boulder, Colorado 80309

\begin{abstract}
We report the first direct observation of the electromagnetic deflection of high energy electron and positron beams as they pass each other with small impact parameters. Measur ments of the deflection amplitude are found in agreement with theoretical expectations. This phenomenon, which is sensitive both to the relative position of the two beams and to their transverse sizes, bas been used successfully to optimize and rasintain collisions at the interaction point of the SLAC Linear Collider.
\end{abstract}

Submitted to Physical Review Letters

\footnotetext{
"Work supported by the U.S. Department of Energy under contracts DE-AC02-86ER40253, DE-AC02-84ER01112, and DE-AC03-76SF00515.
} 
The SLAC :inear Collider ${ }^{1}$ (SLC) is a novel electron-positron accelerator designed to operate with center-of-mass energies around the mass of the neutral intermediate vector boson $\left(Z^{\circ}\right)$. The frequency of collisions in linear colliders is limited by power considerations to a few hundred Hertz, typically two or three orders of magnitude lower than in storage rings. At these frequencies, achieving interaction rates useful for physics requires focusing the beams to transverse sizes of at most a few microns, and then establishing and maintaining collisions between beams with impact parameters smaller than the beam sizes themselves. One technique which has been proposed ${ }^{2}$ for this purpose is based on measuring the deflections produced in the beam trajectories by the coherent electromagnetic interaction between the beam bunches as they pass near or through each other at the interaction point (IP).

In this letter we report the first observation of beam-beam deflections, which constitutes a crucial step in establishing the viability of the linear collider concept. After deriving the predicted properties of the phenomenon and their dependence on beam parameters, we describe the technique used to measure the deflections. We then turn to a discussion of the experimental results, and conclude with an outline of possible refinements in applying beam-beam deflections to luminosity optimization in linear collide:s.

When two oppositely charged, relativistic beams pass each other, they feel an attractive impulse as a result of their electromagnetic interaction. The resulting deflection in their trajectory is given by $\tan ^{-1}\left(p_{t} / p\right) \approx\left(p_{t} / p\right)$, where $p_{t}$ is the transverse momentum imparted to one bunch as it passes through the field of the other bunch, and $p$ is the longitudinal momentum of the bunch. 
Consider the deflection of a single "probe" particle in the fielc of an oppositely charged "target" bunch of $N_{t}$ particles, each with charge $q$, having a Gaussian charge distribution:

$$
G\left(x, y, z, \sigma_{z}, \sigma_{y}, \sigma_{z}\right)=\frac{N_{t} q}{(2 \pi)^{3 / 2} \sigma_{z} \sigma_{y} \sigma_{z}} \exp \left\{-\frac{x^{2}}{2 \sigma_{x}^{2}}-\frac{y^{2}}{2 \sigma_{y}^{2}}-\frac{z^{2}}{2 \sigma_{x}^{2}}\right\}
$$

The transverse electric field of the bunch is given by: ${ }^{3}$

$$
E_{x, y}=-\frac{\partial}{\partial x, y}\left[\frac{\Lambda_{i q}}{\sqrt{\pi} 4 \pi c_{0}} \int_{0}^{\infty} d t \frac{\exp \left\{-\frac{x^{2}}{t+2 \sigma_{x}^{2}}-\frac{y^{2}}{t+2 \sigma_{y}^{2}}-\frac{z^{2}}{t+2 \sigma_{z}^{2}}\right\}}{\left(t+2 \sigma_{z}^{2}\right)^{1 / 2}\left(t+2 \sigma_{y}^{2}\right)^{1 / 2}\left(t+2 \sigma_{z}^{2}\right)^{1 / 2}}\right]
$$

We now solve for the transverse momentum in $z$ and $y$ :

$p_{x, y}=\int_{\Delta} q E_{z, y} d \tau=\frac{-2 N_{1} q^{2} \Delta_{x, y}}{4 \pi \epsilon_{0} c} \int_{0}^{\infty} d t \frac{\exp \left\{-\frac{\Delta_{z}^{2}}{t+2 \sigma_{x}^{2}}-\frac{\Delta_{y}^{2}}{t+2 \sigma_{y}^{2}}\right\}}{\left(t+2 \sigma_{x, y}^{2}\right)\left(t+2 \sigma_{x}^{2}\right)^{1 / 2}\left(t+2 \sigma_{y}^{2}\right)^{1 / 2}}$.

where $\Delta_{T}$ is the length of time the prabe charge is in the field of the beam and $\Delta_{x, y}$ is the offset between the probe charge and the center of the bunch. To obtain the right-hand side of Eq. (3), we have made the substitution $c t=z$ and performed the integration over $z$. Equation 3 is valid when disruption effects, where the beams induce size changes in each other during collision, are negligible. Since $p \approx m_{e} \gamma c$, we may write:

$$
\theta_{x, y}=\frac{-2 r_{e} N_{t} \Delta_{x, y}}{\gamma} \int_{0}^{\infty} d t \frac{\exp \left\{-\frac{\Delta_{x}^{2}}{t+2 \sigma_{x}^{2}}-\frac{\Delta_{y}^{2}}{t+2 \sigma_{y}^{2}}\right\}}{\left(t+2 \sigma_{x, y}^{2}\right)\left(t+2 \sigma_{x}^{2}\right)^{1 / 2}\left(t+2 \sigma_{y}^{2}\right)^{1 / 2}},
$$

where $r_{c}$ is the classical radius of the electron. 
For the realistic ease of a probe beam with a Gaussian charge distribution a convolution with the expression obtained for the deflection of a single particle must be done:

$$
\left\langle\theta_{x, y}\right\rangle=\int_{-\infty}^{\infty} \int_{-\infty}^{\infty} d x d y \tilde{G}\left(x-\bar{x}_{p}, y-\bar{y}_{p}, \sigma_{p, s} \sigma_{p, y}\right) \theta_{x, y}
$$

where $\left\langle\theta_{x, y}\right\rangle$ is the average deflection anjis of the probe bunch (i.e.the deflection of the center of gravity of the bunch). The density distribution of the probe beam is $\hat{G}$, where $\bar{x}_{p}\left(\bar{y}_{p}\right)$ is the center and $\sigma_{p, x}\left(\sigma_{p, y}\right)$ is the standard deviation in the $x(y)$ direction. If we assume that the two beam spots are erect ellipses in the transverse plane, then ${ }^{1}$

$$
\left\langle\theta_{x, y}\right\rangle=\frac{-2 r_{e} N_{t} \Delta_{x, y}}{\gamma} \int_{0}^{\infty} d t \frac{\exp \left\{-\frac{\Delta_{x}^{2}}{\left(t+2 \Sigma_{x}^{2}\right)}-\frac{\Delta_{y}^{2}}{\left(t+2 \Sigma_{y}^{2}\right)}\right\}}{\left(t+2 \Sigma_{x, y}^{2}\right)\left(t+2 \Sigma_{x}^{2}\right)^{1 / 2}\left(t+2 \Sigma_{y}^{2}\right)^{1 / 2}},
$$

where $\Delta_{x}\left(\Delta_{y}\right)$ is now the distance between beam centers and $\Sigma_{x}^{2}=\sigma_{p, x}^{2}+\sigma_{t, x}^{2}$ $\left(\Sigma_{y}^{2}=\sigma_{p, y}^{2}+\sigma_{t, y}^{2}\right)$ is the sum of the squares of the probe and target beam sizes. The integration in this expression can be performed analytically if we assume that $\Sigma_{x}=\Sigma_{y}=\Sigma$ (this includes the case of round probe and target beams). With $\Delta=\left(\Delta_{x}^{2}+\Delta_{y}^{2}\right)^{1 / 2}$ the result is:

$$
\left\langle\theta_{x, y}\right\rangle=\frac{-2 r_{e} N_{t} \Delta_{x, y}}{\gamma \Delta}\left(\frac{1-\exp \left\{-\frac{\Delta^{2}}{2 \Sigma^{2}}\right\}}{\Delta}\right)
$$

Equation 7 shows, as expected, that there is no deflection either when the beams are far from each other or when they are exactly centered. The maximum 
deflection for round beams occurs when the impact parameter is approximately $1.6 \Sigma$. During its start tp phase, the SLC ran with typical beam intengities of slightly less than $1 \times 10^{10}$ particles/pulse, and transverse beam sizes at the IP of approximately $5 \mu \mathrm{m}$, so the maximum deffections seen in this data should be about $35 \mu \mathrm{rad}$. At these sizes and intensities disruption effects are expected to be negligible.

We used four beam position monitors (BPMs), two on either side of the IP, to determine the beam deflection at the IP. These BPMs ${ }^{5}$ are captured between quadrupole magnet pole pieces due to space limitations (Fig. 1). The four electrodes in each BPM are carefully impedance-matched and are read out on both ends into custom-designed electronics. This allows us to measure the vertical and horizontal positions of both beams on the same machine pulse, even though the beams are separated by less than 30 nsec in the BPMs closest to the IP. The pulseto-pulse resolution of these BPMs is measured to be better than $10 \mu \mathrm{m}$ for beam intersities of $\sim 5 \times 10^{9}$ particles/pulse, and is expected to improve further as SLC beam intensitics increase.

Measuring positions in two BPMs on both sides of the IP independently defines incoming and outgoing beam trajectories at the IP. The information from all four BPMs is used in four separate linear fits which yield the beam position, the incoming beam angle, and the beam deflection angle (all evaluated at the IP) in each plane for both beams on a single beam pulse. While the positions and angles of the beams at the IP are observed to be stable on a pulse-by-pulse basis to a fraction of the measured beam size and angular divergence (typically several $\vdash$ indred $\mu \mathrm{rad})$, fluctuations of this magnitude in the outgoing beam angle can still be several times larger than the expected maximum deflection. Fitting directly 
for the difference between the outgoing and incoming angles of a given beam effectively decouples any angular motion of the incoming beam from the deflection angle measurement. This can also be shown to reduce any static misalignments in t!e BPMs to a constant offset of the measured deflection angle, which can be ignored for our applications.

Ij practice, the deflection angles for both beams are measured as a function of the impact parameter as one beam is swept across the other in either the horizontal or the vertical direction. These beam scans are accomplished using small air-core dipole magnets (Fig. 1), which can increment the beam position, between machine pulses, with a resolution of $0.05 \mu \mathrm{m}$. Since the beams deflect each other very little when they are far apart, they must first be brought to within a few beam radii of each other. This has been done by steering the beams, one at a time, onto carbon filaments with radii comparable to the beam size, which are inserted into the center of the beam pipe ai the IP. ${ }^{7,8}$ These filaments produce secondary emission and Bremsstrahlung signals proportional to the fraction of the beam intercepted. The filaments, which are primarily used to measure and optimize transverse beam sizes, stay retracted during deflection measurements.

To get the information necessary to precisely center the beams, one beam is scanned past the other, typically over a range of $\pm 40 \mu \mathrm{m}$ in $2 \mu \mathrm{m}$ steps. The BPM signals are read out on each pulse, processed, and stored by a microcomputer until the scan is finished. The microcomputer also sets and reads back the current in the air-core dipole magnets used to position the besms. When the scan is finished, the microcomputer sends the scan data to a VAX-8800 computer on which the data is analysed and displayed. The results of a typical positron beam scan in $x$ are shown in Fig. 2. The deflection angles parallel $\left(\left\langle\theta_{z}\right\rangle\right)$ and perpendicular $\left(\left\langle\theta_{y}\right\rangle\right)$ to the scan 
direction as a function of the scanned beam's distance from its original position are shown in Figs. 2(a) and 2(b), respectively. For approximately round bearns the data are expected to be described by Eq. (7). For real-time beam centering, fits to the data were approximated using Eq. (7) for the in-plane deflection curve, assuming the beams were aligned in the scanned direction, and by a Gaussian for the out-of-plane curve. As can be seen by the curves shown in Fig. 2, the data is consistent with these approximate functional forms, with the maximum out-ofplane deflection occurring at the zero-crossing of the in-plane denlection curve.

Figure 3 shows a scan with one of the largest maximum deflections measured to date. After centering the beams in $x$, the $\mathrm{e}^{+}$beam was scanned past the $\mathrm{e}^{-}$beam in the $y$ direction. The beam sizes measured prior to this scan using the carbon filaments were $\sigma_{z}=7.2 \mu \mathrm{m}, \sigma_{y}=3.9 \mu \mathrm{m}$ for the electron beam; $\sigma_{x}=4.9 \mu \mathrm{m}, \sigma_{v}=$ $3.9 \mu \mathrm{m}$ for the positron beam. The curve derived using these measured beam sizes as input overlays the data. The beam currents, which provide a multiplicative normalization, were adjusted to give the best fit and were found to be consistent with currents measured by other means. The agreement between data and theory indicates that beam-beam deflections are well understood and reliably measured.

Beam-beam deflections are a powerful tool for luminosity optimization at the SLC. Positioning the scanned beam on the zero-crossing of the deflection curve aligns the beams to a small fraction of the beam size. This has been the primary method used to steer the SLC beams into collision. In most cases, the beam spots at the interaction point are approximately round, so that meaningful fits to the deflection data using the form of Eq. (7) can be made. These immediately yield estimates of the beam sizes and intensities. In the future, pulse-to-pulse sampling 
of the beam deflection will be made in a feedback microcomputer which will be used to compensate for any slow difts of the beams relative to each other.

Beam-beam deflections may also be used to minimize the spot size at the IP. Small changes in beam size are signalled by measurable changes in the slope of the deflection curve through the zero-crossing point. This is demonstrated in Fig. $\ddagger$ which shows the dependence of the slope on the size of the $\mathrm{e}^{-}$beam in $I$ for several different $e^{+}$beam radii. The electron beam size in $y$ is assumed fixed near its optimal value.

The relationship between the slopes of the defection curves and the luminosity is: 9

$$
t=\frac{N f \gamma}{4 \pi r_{e}}\left(S_{z}+S_{y}\right)
$$

where $S_{x}$ and $S_{y}$ are the slopes of the deflection curves measured with separate $x$ and $y$ scans after the bearns bave been centered. The repetition rate of the collider is $f$. An independent measurement of the numbet of particles, $N$, in the deflected beam is also required. The advantage in using the slopes is that they can be accurately measured even when the beams are not round. Optimal luminosity can be achieved by adjusting the focus of the beams to obtain the maximum slopes. Once this has been established, it can be monitored by checking the slopes periodically with short scans across the zero crossing point.

In conclusion, we have presented measurements of the deflections of high-energy electron and positron beams as they pass by each other. These measured deflections agree with theoretical expectations. We have discussed how the deflections have 
been used to star micron-sized beams into co' ision, and how they will be used for spot size optimization at the SLC.

We would like to thank G. B. Bowden, J.-C. Denard, A. Gromme, J.-L. Pellegrin, and M. Ross, who were responsible for the BPM system. The performance of these devices was critical for the results presented here. We also acknowledge the numerous and invaluable contributions to this experiment by the SLC staff and the Final Focus commissioning group. 


\section{REFERENCES}

(a) Present adress: Laboratojre de l'Accélérateur Linóaire, Bàt. 200, Orsay, France 9140.5 .

1. B. Richter and R. Stiening, in Proc. of the 1987 Int. Sym. on Lepton and Photon Intentetions at High Energies, Hamburg, July 27-31, 1987, NorthHolland, Amsterdam, p. 495.

2. P. Bambade and R. Erickson, in 1986 Linear Accelerator Conf. Proc., June 2-6, 1986, SLAC-Report-303, Stanford Linear Accelerator Center, Stanford, California, p. 475.

3. See, for example, K. Takayame, Lett. Cimento 34, 190 (1982).

4. K. Hirata, Nucl. Instrum. Methods A 289, 7 (1988).

5. 3.-C. Desard et al, in Proc. of the 1987 IEEE Particle Accelerator Conf., Washington, D. C., March 16-19, 1987, p. 686.

6. W. A. Koska and S. R. Wagner, SLAC CN-365 (unpublished), August 1988, Stanford Linear Accelerator Center, Stanford, California.

7. R. Fulton et al., Nucl. Instrum. Methods A 274, 37 (1989).

8. G. Bowden et al., SLAC-PUB-4744, submitted to Nucl. Instrum. Methods.

9. W. A. Koska, to be published. 


\section{FIGURE CAPTIONS}

Fig. 1. Schematic of beamline components relevant to the beam-beam deflection measurement.

Fig. 2. A beam-beam deflection scan of the $\mathrm{e}^{+}$beam relative to its initial position showing (a) the in-plane defection, and (b) the out-of-plane deflection. The $e^{-}$beam intensity was about $7 \times 10^{9} e^{-} /$pulse, and both bearns were approximately round with $\sigma=7 \mu \mathrm{m}$. The beams were approximately $10 \mu \mathrm{m}$ apart in the out-of-plane direction during this scan.

Fig. 3. An $e^{+}$beam deflection scan in $y$ after alignment in $x$. The curve overlaying the data is a theoretical calculation using as input the beam sizes as measured by the wire filaments.

Fig. 4. The expected slope of the in-plane deflection curve at the zerocrossing point as a function of the $e^{-}$beam $\sigma_{x}^{-}$with a fixed $\sigma_{y}^{-}$ of $1.5 \mu \mathrm{m}$. The solid curve corresponds to a round $e^{+}$beam with $\sigma^{+}=1.5 \mu \mathrm{m}$, the dashed to $\sigma^{+}=3.0 \mu \mathrm{m}$ and the dotted to $\sigma^{+}=6.0 \mu \mathrm{m}$. The target beam intensity for these calculations was $1 \times 10^{10}$. 

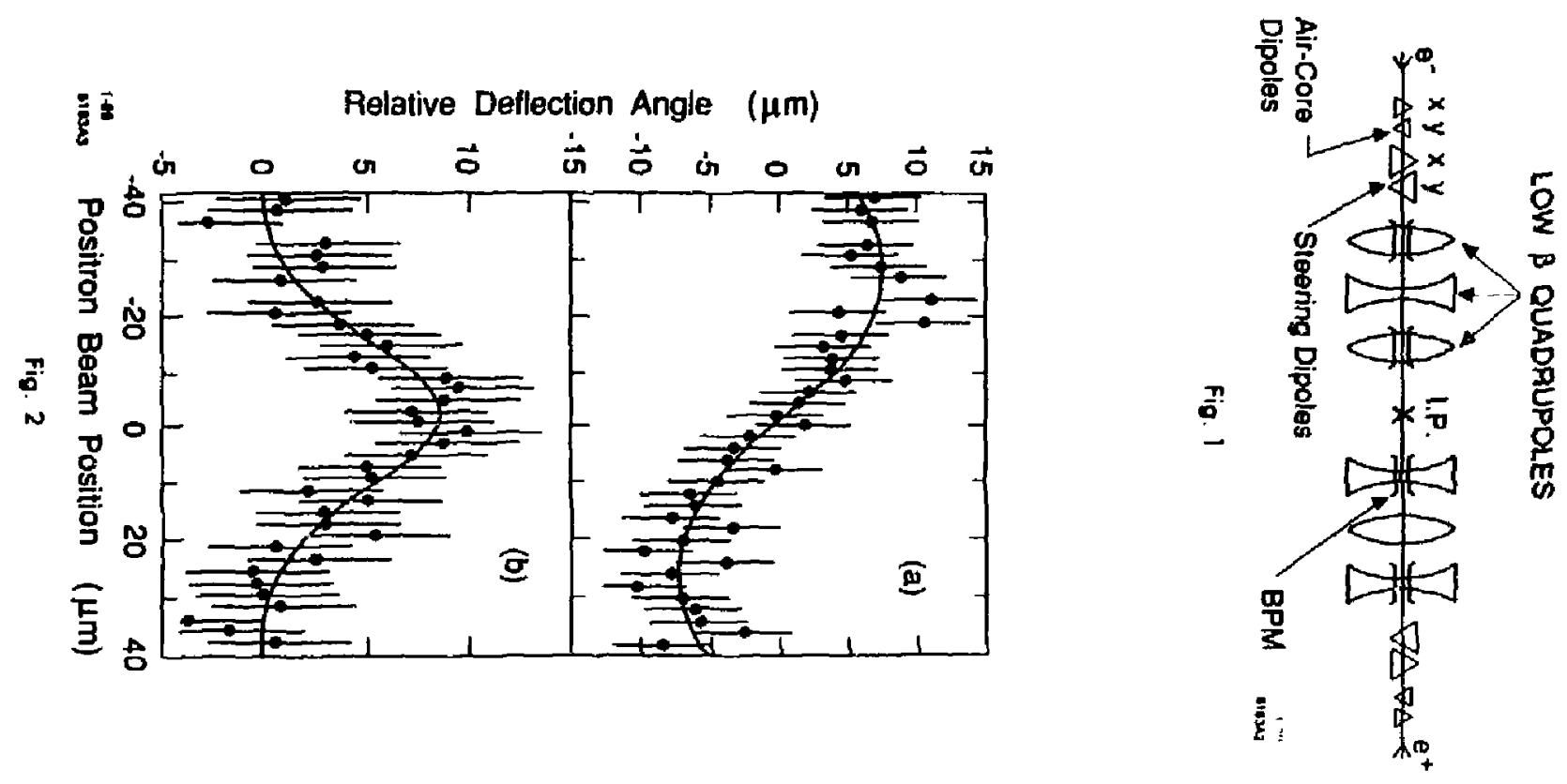


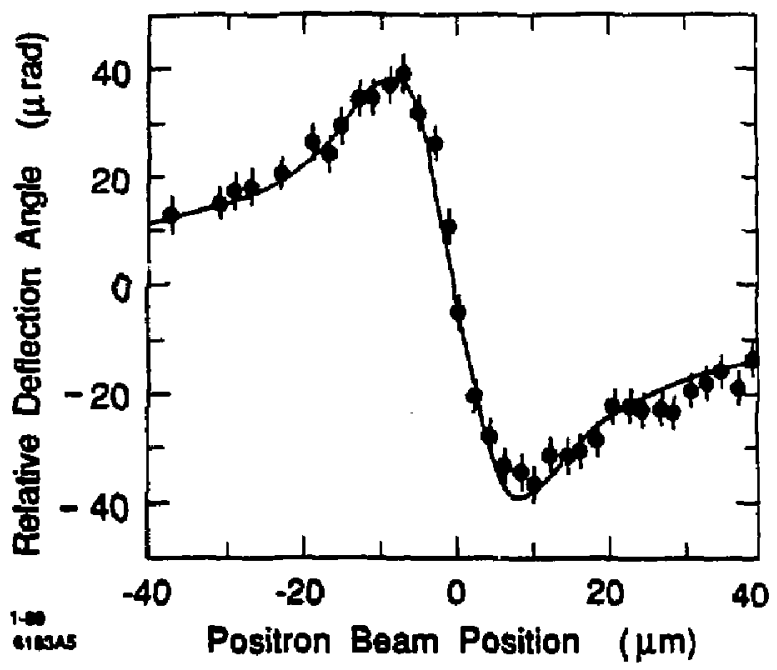

Fig. 3

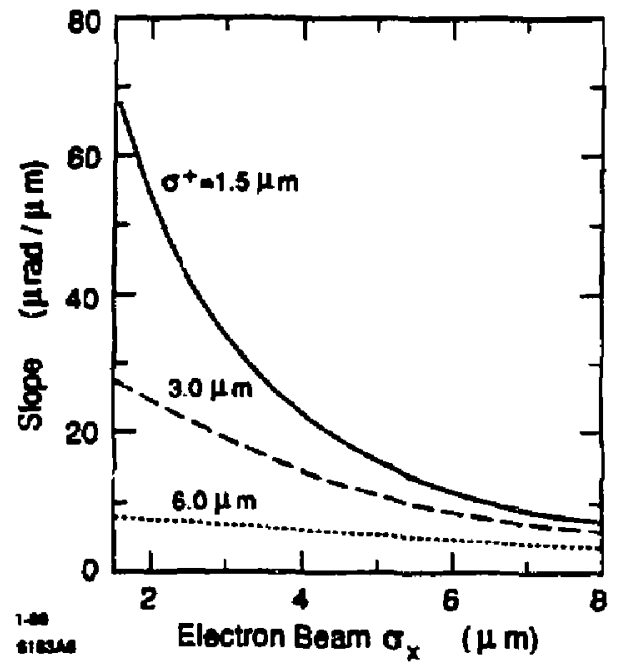

Fig. 4 


\section{REFERENCES}

[1] B. Richter, Nucl. Inst. Meth. 186, 47 (1976).

[2] M. Tigner, Nuovo Cimento 87, 1228 (1965), and B. Richter, SLAC-PUB-4482 (1987).

[3] M. Sands, SLAC-121-Addendum (1979).

[4] SLAC-REPnRT-229 (1980), and SLC Design Handbook (1984).

[5] J. C. Sheppard et al, Commissioning of the SLC Injector, Proceedings of the Particle Accelerator Conference (Washington, D. C., March 1987).

[6] G. E. Fischer et al. A $1.2 \mathrm{GeV}$ Damping Ring Complex for the Stanford Linear Collider, Proceeding of the Twelfe International Conference on High Energy Accelerators, FNAL, 37 (1983).

[7] J. T. Seeman et al., Experimental Beam Dynamics in the SLC Linac, Proceedings of the Particle Accelerator Conference (Washington, D.C., March 1987).

[8] Fatin Bulos et al, Design of a Hign Yield Positron Source, IEEE Trans. Nucl. Sci. NS-32, 1832 (1985), and S. Ecklund, The Stanford Lineer Collider Positron Source, Proceedings of the Workshop on Intense Positron Beams (Idabo Falls, Idaho, June 1987).

[9] G. E. Fischer et al., Some Experiences from the Commissioning Program of the SLC Arc Transport System, Proceedings of the Particle Accelerator Conference (Washington D. G. March 1987).

[10] J. J. Murray et al., The Completed Design of the SLC Final Focus System, Proceedings of the Particle Accelerator Conference (Washington, D. C., March 1987).

[11] S. Kheifets et al., SLAC-YUB-4013 (1987).

[12] R. H. Helm and H. Wiedemann, PEP-Note-303 (1979).

[13] K. L. Brown, SLAC-PUB-2257 (197y).

[14] K. L. Brown, SLAC-PUB-4159 (1987), and R. Erickson, SLAC-PUB-4479 (1987).

[15] K. L. Brown et al. SLAC-91, Rev. 2, May 1977.

[16] The first of the references in [14].

[17] J. J. Murray and T. Fieguth, MURTLE, Private Program. 
[18] K. Bane, Wake-field Effects in a Linear Collider, IEEE Trans. Nucl. Sci. NS-32, $1662(1985)$.

[19] E. D. Courant and H. S. Snyder, Ann. of Phys. 3, 1-48 (1958)

[20] H. Goldstein, Mécanique Classique, Presse Universitaire de Franse. (1964).

[21] L. Rivkin, Damping Ring for the SLAC Linear Collider, PhD Thesis, CalTech (1986).

[22] See for example: B. Richter et R. Stiening, SLAC-PUB-437G (1987),

[23] Jacques Haissinski, A technique for Correcting the Betatron Tunes of the SLC Arcs, Presented at the Particle Acceleral or Conference, Chicago (1989).

(24] M. Sands and N. Toge, Private Communication, (Summer 1987) 